\title{
BLOCK GENERALIZED LOCALLY TOEPLITZ SEQUENCES: THEORY AND APPLICATIONS IN THE UNIDIMENSIONAL CASE*
}

\author{
GIOVANNI BARBARINO ${ }^{\dagger}$, CARLO GARONI $^{\ddagger}$, AND STEFANO SERRA-CAPIZZANO ${ }^{\S}$
}

\begin{abstract}
In computational mathematics, when dealing with a large linear discrete problem (e.g., a linear system) arising from the numerical discretization of a differential equation (DE), knowledge of the spectral distribution of the associated matrix has proved to be useful information for designing/analyzing appropriate solvers-especially, preconditioned Krylov and multigrid solvers-for the considered problem. Actually, this spectral information is of interest also in itself as long as the eigenvalues of the aforementioned matrix represent physical quantities of interest, which is the case for several problems from engineering and applied sciences (e.g., the study of natural vibration frequencies in an elastic material). The theory of generalized locally Toeplitz (GLT) sequences is a powerful apparatus for computing the asymptotic spectral distribution of matrices $A_{n}$ arising from virtually any kind of numerical discretization of DEs. Indeed, when the mesh-fineness parameter $n$ tends to infinity, these matrices $A_{n}$ give rise to a sequence $\left\{A_{n}\right\}_{n}$, which often turns out to be a GLT sequence or one of its "relatives", i.e., a block GLT sequence or a reduced GLT sequence. In particular, block GLT sequences are encountered in the discretization of systems of DEs as well as in the higher-order finite element or discontinuous Galerkin approximation of scalar/vectorial DEs. This work is a review, refinement, extension, and systematic exposition of the theory of block GLT sequences. It also includes several emblematic applications of this theory in the context of DE discretizations.
\end{abstract}

Key words. asymptotic distribution of singular values and eigenvalues, block Toeplitz matrices, block generalized locally Toeplitz matrices, numerical discretization of differential equations, finite differences, finite elements, isogeometric analysis, discontinuous Galerkin methods, tensor products, B-splines.

AMS subject classifications. 15A18, 15B05, 47B06, 65N06, 65N30, 65N25, 15A60, 15A69, 65D07

\section{Contents}

\section{Introduction}

1.1 GLT sequences: the tool for computing the spectral distribution of DE discretization matrices . . . . . . . . . . . . . . . . . . . 30

1.2 Practical uses of the spectral distribution . . . . . . . . . . . . . . . 31

1.3 Key ideas behind the notion of GLT sequences . . . . . . . . . . . . . . 32

1.4 Contributions and structure of the present work . . . . . . . . . . . . 37

2 Mathematical background 38

2.1 Notation and terminology . . . . . . . . . . . . . . . . . . . . 38

2.2 Preliminaries on matrix analysis $\ldots \ldots \ldots \ldots \ldots$

2.2 .1 Matrix norms . . . . . . . . . . . . . . . . . . . . . . 40

2.2.2 Tensor products and direct sums . . . . . . . . . . . . . . . . . 40

2.3 Preliminaries on measure and integration theory . . . . . . . . . . . . . . 41

2.3.1 Measurability . . . . . . . . . . . . . . . . 41

2.3.2 Essential range of matrix-valued functions . . . . . . . . . . . . 42

2.3.3 $L^{p}$-norms of matrix-valued functions . . . . . . . . . . . 43

2.3.4 Convergence in measure and the topology $\tau_{\text {measure }} \ldots \ldots$. . . . . . 43

${ }^{*}$ Received August 12, 2019. Accepted November 6, 2019. Published online on January 30, 2020. Recommended by L. Reichel. Carlo Garoni acknowledges the MIUR Excellence Department Project awarded to the Department of Mathematics, University of Rome "Tor Vergata", CUP E83C18000100006, and the support obtained by the Beyond Borders Programme of the University of Rome "Tor Vergata" through the project ASTRID, CUP E84I19002250005.

${ }^{\dagger}$ Faculty of Sciences, Scuola Normale Superiore, Italy (giovanni.barbarino@sns. it).

${ }^{\ddagger}$ Department of Mathematics, University of Rome "Tor Vergata", Italy; Department of Science and High Technology, University of Insubria, Italy (garoni@mat. uniroma2. it; carlo.garoni@uninsubria.it).

${ }^{\S}$ Department of Humanities and Innovation, University of Insubria, Italy; Department of Information Technology, Uppsala University, Sweden (stefano. serrac@uninsubria.it; stefano. serra@it.uu.se). 
2.3.5 Riemann-integrable functions . . . . . . . . . . . . . 45

2.4 Singular value and eigenvalue distribution of a sequence of matrices . . . . . 46

2.4.1 The notion of singular value and eigenvalue distribution . . . . . . . 46

2.4 .2 Clustering and attraction . . . . . . . . . . . . . . . 48

2.4 .3 Zero-distributed sequences . . . . . . . . . . . . . . . . . 50

2.4.4 Sparsely unbounded and sparsely vanishing sequences of matrices . . . . 50

2.4.5 Spectral distribution of sequences of perturbed/compressed/expanded Hermitian matrices . . . . . . . . . . . . . . . . . 51

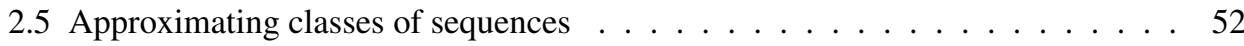

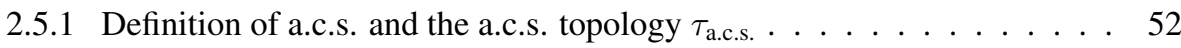

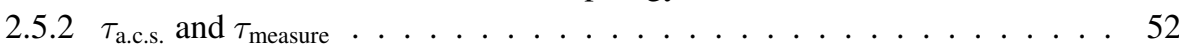

2.5.3 The a.c.s. tools for computing singular value and eigenvalue distributions 54

2.5 .4 The a.c.s. algebra . . . . . . . . . . . . . . . . . 54

2.5.5 A criterion to identify a.c.s. . . . . . . . . . . . . . . . 54

2.6 Block Toeplitz matrices . . . . . . . . . . . . . . . . . . . 55

2.7 Block diagonal sampling matrices $\ldots \ldots \ldots \ldots$

3 Block locally Toeplitz sequences $\quad 57$

3.1 The block LT operator . . . . . . . . . . . . . . . . . . . . . . . 57

3.2 Definition of block LT sequences . . . . . . . . . . . . . . . . 61

3.3 Fundamental examples of block LT sequences . . . . . . . . . . . . . . 61

3.3 .1 Zero-distributed sequences $\ldots \ldots \ldots \ldots$. . . . . . . . . 61

3.3.2 Sequences of block diagonal sampling matrices . . . . . . . . 61

3.3.3 Block Toeplitz sequences . . . . . . . . . . . . . . . . . . . . . 64

3.4 Singular value and spectral distribution of sums of products of block LT

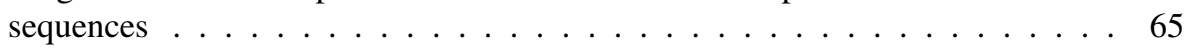

3.5 Algebraic properties of block LT sequences . . . . . . . . . . . . . . 66

3.6 Characterizations of block LT sequences . . . . . . . . . . . . . . . . 67

4 Block generalized locally Toeplitz sequences $\quad 68$

4.1 Equivalent definitions of block GLT sequences . . . . . . . . . . . . . . . . . 69

4.2 Singular value and spectral distribution of block GLT sequences . . . . . . . 70

4.3 Block GLT sequences and matrix-valued measurable functions . . . . . . . . 72

4.4 The block GLT algebra . . . . . . . . . . . . . . . . . . . 73

4.5 Topological density results for block GLT sequences . . . . . . . . . . . . . 75

4.6 Characterizations of block GLT sequences . . . . . . . . . . . . . . . 77

4.7 Sequences of block diagonal sampling matrices . . . . . . . . . . . . . 77

4.8 Sequences of block matrices with block GLT blocks . . . . . . . . . . . . 78

4.9 Further possible definitions of block GLT sequences . . . . . . . . . . . . 80

5 Summary of the theory $\quad 80$

6 Applications $\quad 86$

6.1 FD discretization of systems of DEs . . . . . . . . . . . . . . 86

6.2 Higher-order FE discretization of diffusion equations . . . . . . . . . . . . 90

6.3 Higher-order FE discretization of convection-diffusion-reaction equations . . 98

6.4 Higher-order FE discretization of systems of DEs . . . . . . . . . . . . . 100

6.5 Higher-order isogeometric Galerkin discretization of eigenvalue problems . . 105 
1. Introduction. The theory of generalized locally Toeplitz (GLT) sequences stems from Tilli's work on locally Toeplitz (LT) sequences [79] and from the spectral theory of Toeplitz matrices [2, 21, 22, 23, 24, 59, 64, 80, 82, 83, 84]. It was then carried forward in $[50,51,75,76]$, and it has been recently extended in $[3,4,5,6,7,8,55,56]$. It is a powerful apparatus for computing the asymptotic spectral distribution of matrices arising from the numerical discretization of continuous problems, such as integral equations (IEs) and, especially, differential equations (DEs). Experience reveals that virtually any kind of numerical method for the discretization of DEs gives rise to structured matrices $A_{n}$ whose asymptotic spectral distribution, as the mesh-fineness parameter $n$ tends to infinity, can be computed through the theory of GLT sequences. There exist many other applications of this theory including, e.g., the analysis of signal decomposition methods [28] and geometric means of matrices [50, Section 10.3], but the computation of the spectral distribution of DE discretization matrices remains undoubtedly the main application. In Section 1.1, we take an overview of this main application. In Section 1.2, we describe some practical uses of the spectral distribution. In Section 1.3, we illustrate the key ideas behind the notion of GLT sequences, with a special attention to the so-called block GLT sequences, so as to give readers the flavor of what we are going to deal with in this work. In Section 1.4, we outline the contributions and the structure of the present work.

1.1. GLT sequences: the tool for computing the spectral distribution of DE discretization matrices. Suppose we are given a linear DE, say

$$
\mathcal{A} u=g,
$$

with $\mathcal{A}$ denoting the associated differential operator, and suppose we want to approximate the solution of such DE by means of a certain (linear) numerical method. In this case, the actual computation of the numerical solution reduces to solving a linear system

$$
A_{n} \mathbf{u}_{n}=\mathbf{g}_{n},
$$

whose size $d_{n}$ increases with $n$ and ultimately tends to infinity as $n \rightarrow \infty$. Hence, what we actually have is not just a single linear system but a whole sequence of linear systems with increasing size, and what is often observed in practice is that the sequence of discretization matrices $A_{n}$ enjoys an asymptotic spectral distribution, which is somehow connected to the spectrum of the differential operator $\mathcal{A}$ associated with the DE. More precisely, it often happens that, for a large set of test functions $F$ (usually, for all continuous functions $F$ with bounded support), the following limit relation holds:

$$
\lim _{n \rightarrow \infty} \frac{1}{d_{n}} \sum_{j=1}^{d_{n}} F\left(\lambda_{j}\left(A_{n}\right)\right)=\frac{1}{\mu_{k}(D)} \int_{D} \frac{\sum_{i=1}^{s} F\left(\lambda_{i}(\kappa(\mathbf{y}))\right)}{s} \mathrm{~d} \mathbf{y},
$$

where $\lambda_{j}\left(A_{n}\right), j=1, \ldots, d_{n}$, are the eigenvalues of $A_{n}, D$ is a subset of some $\mathbb{R}^{k}, \mu_{k}(D)$ is the $k$-dimensional volume of $D, \kappa: D \subset \mathbb{R}^{k} \rightarrow \mathbb{C}^{s \times s}$, and $\lambda_{i}(\kappa(\mathbf{y})), i=1, \ldots, s$, are the eigenvalues of the $s \times s$ matrix $\kappa(\mathbf{y})$. In this situation, the matrix-valued function $\kappa$ is referred to as the spectral symbol of the sequence $\left\{A_{n}\right\}_{n}$. The spectral information contained in $\kappa$ can be informally summarized as follows: assuming that $n$ is large enough, the eigenvalues of $A_{n}$, except possibly for a small portion of outliers, can be subdivided into $s$ different subsets of approximately the same cardinality, and the eigenvalues belonging to the $i$ th subset are approximately equal to the samples of the $i$ th eigenvalue function $\lambda_{i}(\kappa(\mathbf{y}))$ over a uniform grid in the domain $D$. For instance, if $k=1, d_{n}=n s$, and $D=[a, b]$, then, assuming we 
have no outliers, the eigenvalues of $A_{n}$ are approximately equal to

$$
\lambda_{i}\left(\kappa\left(a+j \frac{b-a}{n}\right)\right), \quad j=1, \ldots, n, \quad i=1, \ldots, s,
$$

for $n$ large enough. Similarly, if $k=2, d_{n}=n^{2} s$, and $D=\left[a_{1}, b_{1}\right] \times\left[a_{2}, b_{2}\right]$, then, assuming we have no outliers, the eigenvalues of $A_{n}$ are approximately equal to

$$
\lambda_{i}\left(\kappa\left(a_{1}+j_{1} \frac{b_{1}-a_{1}}{n}, a_{2}+j_{2} \frac{b_{2}-a_{2}}{n}\right)\right), \quad j_{1}, j_{2}=1, \ldots, n, \quad i=1, \ldots, s,
$$

for $n$ large enough. It is then clear that the symbol $\kappa$ provides a "compact" and quite accurate description of the spectrum of the matrices $A_{n}$ (for $n$ large enough).

The theory of GLT sequences is a powerful apparatus for computing the spectral symbol $\kappa$. Indeed, the sequence of discretization matrices $\left\{A_{n}\right\}_{n}$ turns out to be a GLT sequence with symbol (or kernel) $\kappa$ for many classes of DEs and numerical methods, especially when the numerical method belongs to the family of the so-called "local methods". Local methods are, for example, finite difference methods, finite element methods with "locally supported" basis functions, and collocation methods; in short, all standard numerical methods for the approximation of DEs. Depending on the considered DE and numerical method, the sequence $\left\{A_{n}\right\}_{n}$ might be a scalar GLT sequence (that is, a GLT sequence whose symbol $\kappa$ is a scalar function $)^{1}$ or a block/reduced GLT sequence. In particular, block GLT sequences are encountered in the discretization of vectorial DEs (systems of scalar DEs) as well as in the higher-order finite element or discontinuous Galerkin approximation of scalar DEs. We refer the reader to [50, Section 10.5], [51, Section 7.3], and [20, 49, 75, 76] for applications of the theory of GLT sequences in the context of finite difference (FD) discretizations of DEs; to [50, Section 10.6], [51, Section 7.4], and [10, 20, 42, 49, 57, 67, 76] for the finite element (FE) case; to [12] for the finite volume (FV) case; to [50, Section 10.7], [51, Sections 7.5-7.7], and $[36,45,46,47,48,52,57,68]$ for the case of isogeometric analysis (IgA) discretizations, both in the collocation and Galerkin frameworks; and to [40] for a further application to fractional DEs. We also refer the reader to [50, Section 10.4] and [1, 72] for a look at the GLT approach for sequences of matrices arising from IE discretizations.

1.2. Practical uses of the spectral distribution. It is worth emphasizing that the asymptotic spectral distribution of DE discretization matrices, whose computation is the main objective of the theory of GLT sequences, is not only interesting from a theoretical viewpoint but can also be used for practical purposes. For example, it is known that the convergence properties of mainstream iterative solvers, such as multigrid and preconditioned Krylov methods, strongly depend on the spectral features of the matrices to which they are applied. The spectral distribution can then be exploited to design efficient solvers of this kind and to analyze/predict their performance. In this regard, we recall that noteworthy estimates on the superlinear convergence of the conjugate gradient method obtained by Beckermann and Kuijlaars in [9] are closely related to the asymptotic spectral distribution of the considered matrices. More recently, in the context of Galerkin and collocation IgA discretizations of elliptic DEs, the spectral distribution computed through the theory of GLT sequences in a series of papers $[36,45,46,47,48]$ was exploited in [34, 35, 37] to devise and analyze optimal and robust multigrid solvers for IgA linear systems. In addition to the design and analysis of appropriate solvers, the spectral distribution of DE discretization matrices is of interest also in itself whenever the eigenvalues of such matrices represent physical quantities of interest. This is

\footnotetext{
${ }^{1}$ A scalar GLT sequence is a GLT sequence in the classical sense of this word, and it is usually referred to as a GLT sequence without further specifications.
} 
the case for a broad class of problems arising in engineering and applied sciences, such as the study of natural vibration frequencies for an elastic material; see the review [57] and the references therein.

1.3. Key ideas behind the notion of GLT sequences. Following Tilli [80], in this section we tell the story that led to the birth of LT sequences, that is, the eminent ancestors of GLT sequences. Special attention is devoted to understanding the reason why it was necessary to go beyond classical (scalar) LT sequences and introduce the notion of block LT sequences. Our main purpose here is to illustrate the key ideas behind the notions of LT and block LT sequences, without entering into technical details. For this reason, the forthcoming discussion will be quite informal and, in particular, we will not provide justifications to all the assertions we will make. Precise mathematical definitions and proofs will come only later on in this work.

As it is known, a Toeplitz matrix is a matrix whose entries are constant along each diagonal. Matrices with a Toeplitz-related structure arise in many different areas of pure and applied mathematics whenever one deals with a problem that has some kind of translation invariance. For example, they are encountered

when dealing with Markov chains [15, 29, 63], subdivision algorithms [65], Riccati equations [14], reconstruction of signals with missing data [32], inpainting problems [26], and, of course, numerical discretizations of constant-coefficient DEs; see [50, 51] and the references therein. Any function $f \in L^{1}([-\pi, \pi])$ generates a sequence of Toeplitz matrices $T_{n}(f)=\left[f_{i-j}\right]_{i, j=1}^{n}$ via its Fourier coefficients

$$
f_{k}=\frac{1}{2 \pi} \int_{-\pi}^{\pi} f(\theta) e^{-\mathrm{i} k \theta} \mathrm{d} \theta, \quad k \in \mathbb{Z}
$$

The asymptotic distribution of the singular values and eigenvalues of $T_{n}(f)$ has been completely characterized in terms of the generating function $f$. More specifically, for all continuous functions $F$ with bounded support we have

$$
\lim _{n \rightarrow \infty} \frac{1}{n} \sum_{i=1}^{n} F\left(\sigma_{i}\left(T_{n}(f)\right)\right)=\frac{1}{2 \pi} \int_{-\pi}^{\pi} F(|f(\theta)|) \mathrm{d} \theta ;
$$

if $f$ is real, we also have

$$
\lim _{n \rightarrow \infty} \frac{1}{n} \sum_{i=1}^{n} F\left(\lambda_{i}\left(T_{n}(f)\right)\right)=\frac{1}{2 \pi} \int_{-\pi}^{\pi} F(f(\theta)) \mathrm{d} \theta .
$$

Equations (1.1)-(1.2) are usually referred to as the Szegő formulas for Toeplitz matrices; see [50, Section 6.5] for their proof.

Now, consider the simple model problem

$$
\left\{\begin{array}{l}
-u^{\prime \prime}(x)=g(x), \quad 0<x<1, \\
u(0)=u(1)=0 .
\end{array}\right.
$$

The discretization of this problem through any reasonable finite difference scheme over a uniform grid of $n$ points leads to the solution of a linear system whose matrix is Toeplitz or "almost" Toeplitz. For example, in the case of the classical 3-point difference scheme 
$(-1,2,-1)$, the resulting discretization matrix is

$$
T_{n}(f)=\left[\begin{array}{ccccc}
2 & -1 & & & \\
-1 & 2 & -1 & & \\
& \ddots & \ddots & \ddots & \\
& & -1 & 2 & -1 \\
& & & -1 & 2
\end{array}\right], \quad f(\theta)=2-2 \cos \theta
$$

What is relevant to our purpose, however, is that the Toeplitz structure of the resulting matrices is a direct consequence of the translation invariance of the differential operator in (1.3), i.e., the second derivative. Note that the translation invariance is clear from the equation $u^{\prime \prime}(x+\tau)=(u(x+\tau))^{\prime \prime}$.

Since differential operators are translation-invariant only when they have constant coefficients, it is not reasonable to expect a Toeplitz structure in a matrix which discretizes a differential operator with nonconstant coefficients. Consider, for instance, the Sturm-Liouville problem

$$
\left\{\begin{array}{l}
-\left(a(x) u^{\prime}(x)\right)^{\prime}=g(x), \quad 0<x<1, \\
u(0)=u(1)=0 .
\end{array}\right.
$$

The generalized version of the $(-1,2,-1)$ scheme leads to the matrix

$$
A_{n}=\left[\begin{array}{ccccc}
a_{\frac{1}{2}}+a_{\frac{3}{2}} & -a_{\frac{3}{2}} & & & \\
-a_{\frac{3}{2}} & a_{\frac{3}{2}}+a_{\frac{5}{2}} & -a_{\frac{5}{2}} & & \\
& -a_{\frac{5}{2}} & \ddots & \ddots & \\
& & \ddots & \ddots & -a_{n-\frac{1}{2}} \\
& & & -a_{n-\frac{1}{2}} & a_{n-\frac{1}{2}}+a_{n+\frac{1}{2}}
\end{array}\right],
$$

where $a_{i}=a\left(\frac{i}{n+1}\right), i=\frac{1}{2}, \frac{3}{2}, \ldots, n+\frac{1}{2}$. Observe that the matrix (1.6) reduces to the Toeplitz matrix (1.4) if $a(x)=1$, that is, when the differential operator has constant coefficients. It is clear, however, that $A_{n}$ is not Toeplitz if $a(x)$ is not constant. Nevertheless, the singular values and eigenvalues of $A_{n}$ are nicely distributed, according to

$$
\lim _{n \rightarrow \infty} \frac{1}{n} \sum_{i=1}^{n} F\left(\sigma_{i}\left(A_{n}\right)\right)=\frac{1}{2 \pi} \int_{0}^{1} \int_{-\pi}^{\pi} F(|a(x) f(\theta)|) \mathrm{d} \theta \mathrm{d} x
$$

and

$$
\lim _{n \rightarrow \infty} \frac{1}{n} \sum_{i=1}^{n} F\left(\lambda_{i}\left(A_{n}\right)\right)=\frac{1}{2 \pi} \int_{0}^{1} \int_{-\pi}^{\pi} F(a(x) f(\theta)) \mathrm{d} \theta \mathrm{d} x,
$$

where $f(\theta)=2-2 \cos \theta$ as in (1.4); for the proof of these formulas, see [50, Section 10.5.1]. Observe that, if $a(x)=1$, then the equations (1.7)-(1.8) reduce to the Szegó formulas (1.1)(1.2) for $T_{n}(f)$. In view of this, equations (1.7)-(1.8) can be thought of as weighted Szegó formulas with $a(x)$ as weight function. If we examine the asymptotic formulas (1.7)-(1.8) 
in more detail, then we see that the distribution of the singular values and eigenvalues is completely determined by two independent functions, namely $a(x)$ and $f(\theta)$. The former comes from the differential problem (1.5), while the latter depends only on the finite difference scheme adopted to discretize the problem (in our case, this is the generalized version of the 3point scheme $(-1,2,-1)$ ). It is natural to ask what happens if a different scheme (for example, a 5-point scheme) is used to discretize problem (1.5): are the singular values and eigenvalues of the resulting matrices still nicely distributed, maybe according to some weighted Szegó formulas like in equations (1.7)-(1.8)? The answer, quite general, is affirmative (see [50, Section 10.5.2] for a discussion of this topic). Going back to (1.6), the sequence of matrices $\left\{A_{n}\right\}_{n}$ turns out to be much more structured than it might be expected: it is what we call a locally Toeplitz sequence (more precisely, it is locally Toeplitz with respect to the weight function $a(x)$ and the generating function $f(\theta)$ ). In order to justify our terminology, we can intuitively argue as follows. A matrix $\left[\alpha_{i, j}\right]_{i, j=1}^{n}$ has Toeplitz structure if $\alpha_{i+1, j+1}=\alpha_{i, j}$ or, equivalently, if its entries are constant along the diagonals. Consider one of the above matrices $A_{n}$, for a large value of $n$ (large, say, with respect to the derivative of $a(x)$ ). If, from any entry of $A_{n}$, we shift downwards by one position along the same diagonal, then the new entry differs from the old one by a quantity which tends to zero as $n$ tends to infinity (the difference is $O(1 / n)$ if, for example, $a(x)$ is Lipschitz continuous over $[0,1])$. Now consider any given diagonal of $A_{n}$ (the main diagonal, for instance). For large $n$, the first element is close to $2 a(0)$, while the last one is close to $2 a(1)$ (and hence $A_{n}$ is not Toeplitz if $a(0) \neq a(1)$ ). Nevertheless, the transition from $2 a(0)$ to $2 a(1)$ along the diagonal is more and more gradual as $n$ increases and, in a sense, we can say that the transition is continuous in the limit (just as the function $2 a(x)$ ). As a consequence, when $n$ is very large with respect to $k$, any principal submatrix of $A_{n}$ made of $k$ consecutive rows and columns possesses a sort of approximate Toeplitz structure.

Another distinguished example of a locally Toeplitz sequence (quite similar to the above but simpler to handle) is given by the sequence of matrices $\left\{B_{n}\right\}_{n}$, where

$$
\begin{aligned}
B_{n} & =\left[\begin{array}{ccccc}
2 a\left(x_{1}\right) & -a\left(x_{1}\right) & & & \\
-a\left(x_{2}\right) & 2 a\left(x_{2}\right) & -a\left(x_{2}\right) & & \\
& \ddots & \ddots & \ddots & \\
& & -a\left(x_{n-1}\right) & 2 a\left(x_{n-1}\right) & -a\left(x_{n-1}\right) \\
& & -a\left(x_{n}\right) & 2 a\left(x_{n}\right)
\end{array}\right] \\
& =D_{n}(a) T_{n}(2-2 \cos \theta),
\end{aligned}
$$

and $D_{n}(a)$ is the diagonal sampling matrix containing the samples of the function $a(x)$ over the uniform grid $x_{i}=\frac{i}{n}, i=1, \ldots, n$,

$$
D_{n}(a)=\operatorname{diag}_{i=1, \ldots, n} a\left(x_{i}\right)=\left[\begin{array}{llll}
a\left(x_{1}\right) & & & \\
& a\left(x_{2}\right) & & \\
& & \ddots & \\
& & & a\left(x_{n}\right)
\end{array}\right] .
$$

Looking at a relatively small submatrix of $B_{n}$ (according to a "local" perspective), one easily recognizes an approximate Toeplitz structure weighted through the function $a(x)$. For instance, the $2 \times 2$ leading principal submatrix

$$
\left[\begin{array}{ll}
2 a\left(x_{1}\right) & -a\left(x_{1}\right) \\
-a\left(x_{2}\right) & 2 a\left(x_{2}\right)
\end{array}\right]
$$


is approximately equal to

$$
a\left(x_{1}\right)\left[\begin{array}{rr}
2 & -1 \\
-1 & 2
\end{array}\right]=a\left(x_{1}\right) T_{2}(2-2 \cos \theta)
$$

because the difference between these two matrices goes to 0 in the spectral norm as $n \rightarrow \infty$. Similarly, if $C_{\lfloor\sqrt{n}\rfloor}$ is a submatrix of size $\lfloor\sqrt{n}\rfloor$ obtained as the intersection of $\lfloor\sqrt{n}\rfloor$ consecutive rows and columns of $B_{n}$, then $C_{\lfloor\sqrt{n}\rfloor} \approx a\left(x_{i}\right) T_{\lfloor\sqrt{n}\rfloor}(2-2 \cos \theta)$, where $a\left(x_{i}\right)$ is any of the evaluations of $a(x)$ appearing in $C_{\lfloor\sqrt{n}\rfloor}$. More precisely, one can prove that

$$
C_{\lfloor\sqrt{n}\rfloor}=a\left(x_{i}\right) T_{\lfloor\sqrt{n}\rfloor}(2-2 \cos \theta)+E_{\lfloor\sqrt{n}\rfloor},
$$

where the error $E_{\lfloor\sqrt{n}\rfloor}$ tends to zero in the spectral norm as $n \rightarrow \infty$ (the norm $\left\|E_{\lfloor\sqrt{n}\rfloor}\right\|$ is proportional to the modulus of continuity of $a$ evaluated at $\lfloor\sqrt{n}\rfloor / n)$. The latter assertion remains true if $\lfloor\sqrt{n}\rfloor$ is replaced by any other integer $k_{n}$ such that $k_{n}=o(n)$. In other words, if we explore "locally" the matrix $B_{n}$ using an ideal microscope and considering a large value of $n$, then we realize that the "local" structure of $B_{n}$ is approximately the Toeplitz structure generated by $2-2 \cos \theta$ and weighted through the function $a(x)$.

So far, we have only discussed classical (i.e., scalar) locally Toeplitz sequences, whose asymptotic singular value and eigenvalue distributions are naturally characterized in terms of scalar functions such as $a(x) f(\theta)$ in (1.7) and (1.8). The remainder of this section is devoted to understanding how block locally Toeplitz sequences enter the scene.

As it is known, an $s$-block Toeplitz matrix (or simply a block Toeplitz matrix if $s$ is clear from the context) is a matrix whose "entries" are constant along each diagonal with the only difference with respect to traditional Toeplitz matrices being the fact that these "entries" are $s \times s$ matrices (blocks). Any function $f:[-\pi, \pi] \rightarrow \mathbb{C}^{s \times s}$ with entries $f_{i j} \in L^{1}([-\pi, \pi])$ generates a sequence of $s$-block Toeplitz matrices $T_{n}(f)=\left[f_{i-j}\right]_{i, j=1}^{n}$ via its Fourier coefficients

$$
f_{k}=\frac{1}{2 \pi} \int_{-\pi}^{\pi} f(\theta) e^{-\mathrm{i} k \theta} \mathrm{d} \theta, \quad k \in \mathbb{Z}
$$

(the integrals are computed componentwise). The asymptotic distribution of the singular values and eigenvalues of $T_{n}(f)$ has been completely characterized in terms of the generating function $f$. More specifically, for all continuous functions $F$ with bounded support, we have

$$
\lim _{n \rightarrow \infty} \frac{1}{d_{n}} \sum_{i=1}^{d_{n}} F\left(\sigma_{i}\left(T_{n}(f)\right)\right)=\frac{1}{2 \pi} \int_{-\pi}^{\pi} \frac{\sum_{i=1}^{s} F\left(\sigma_{i}(f(\theta))\right)}{s} \mathrm{~d} \theta ;
$$

if $f(\theta)$ is Hermitian for every $\theta$, we also have

$$
\lim _{n \rightarrow \infty} \frac{1}{d_{n}} \sum_{i=1}^{d_{n}} F\left(\lambda_{i}\left(T_{n}(f)\right)\right)=\frac{1}{2 \pi} \int_{-\pi}^{\pi} \frac{\sum_{i=1}^{s} F\left(\lambda_{i}(f(\theta))\right)}{s} \mathrm{~d} \theta,
$$

where $d_{n}=n s$ is the size of $T_{n}(f)$. Equations (1.9)-(1.10) are usually referred to as the Szegô formulas for block Toeplitz matrices; see [80] for their proof.

Now, consider the classical Lagrangian $p$-degree finite element discretization of the translation-invariant problem (1.3) on a uniform mesh in $[0,1]$ with stepsize $\frac{1}{n}$. If $p=1$, then the resulting discretization matrix is again a scalar Toeplitz matrix, namely $T_{n-1}(2-2 \cos \theta)$. If $p \geq 2$, then the situation changes. For instance, for $p=2$, the resulting discretization matrix 
is given by ${ }^{2}$

$$
K_{n}^{[2]}(1)=\left[\begin{array}{ccccc}
K_{0} & K_{1}^{T} & & & \\
K_{1} & K_{0} & K_{1}^{T} & & \\
& \ddots & \ddots & \ddots & \\
& & K_{1} & K_{0} & K_{1}^{T} \\
& & & K_{1} & K_{0}
\end{array}\right], \quad K_{0}=\frac{1}{3}\left[\begin{array}{cc}
16 & -8 \\
-8 & 14
\end{array}\right], \quad K_{1}=\frac{1}{3}\left[\begin{array}{cc}
0 & -8 \\
0 & 1
\end{array}\right] .
$$

This is a $2 \times 2$ block Toeplitz matrix deprived of its last row and column. More specifically,

$$
K_{n}^{[2]}(1)=T_{n}\left(f^{[2]}\right)_{-},
$$

where

$$
f^{[2]}(\theta)=K_{0}+K_{1} e^{\mathrm{i} \theta}+K_{1}^{T} e^{-\mathrm{i} \theta}=\frac{1}{3}\left[\begin{array}{cc}
16 & -8-8 e^{\mathrm{i} \theta} \\
-8-8 e^{-\mathrm{i} \theta} & 14+2 \cos \theta
\end{array}\right]
$$

The eigenvalues and singular values of the sequence $\left\{K_{n}^{[2]}(1)\right\}_{n}$ are distributed as those of $\left\{T_{n}\left(f^{[2]}\right)\right\}_{n}$ according to equations (1.9)-(1.10) with $f=f^{[2]}$ and $s=2$. For $p>2$, the situation is completely analogous: the resulting discretization matrix $K_{n}^{[p]}(1)$ is a $p$-block Toeplitz matrix $T_{n}\left(f^{[p]}\right)$ deprived of its last row and column, and the eigenvalues and singular values of $\left\{K_{n}^{[p]}(1)\right\}_{n}$ are distributed as those of $\left\{T_{n}\left(f^{[p]}\right)\right\}_{n}$ according to equations (1.9)(1.10) with $f=f^{[p]}$ and $s=p$.

Let us now consider the same Lagrangian $p$-degree finite element discretization as before but applied this time to the variable-coefficient problem (1.5). For $p=2$, the resulting discretization matrix is given by

$$
\begin{aligned}
K_{n}^{[2]}(a) \approx & {\left[\begin{array}{ccccc}
a\left(\frac{1}{n}\right) K_{0} & a\left(\frac{1}{n}\right) K_{1}^{T} & & & \\
a\left(\frac{2}{n}\right) K_{1} & a\left(\frac{2}{n}\right) K_{0} & a\left(\frac{2}{n}\right) K_{1}^{T} & & \\
& a\left(\frac{3}{n}\right) K_{1} & a\left(\frac{3}{n}\right) K_{0} & a\left(\frac{3}{n}\right) K_{1}^{T} & \\
& \ddots & \ddots & \ddots & \\
& & \ddots & \ddots & a\left(\frac{n-1}{n}\right) K_{1}^{T} \\
& & & a(1) K_{1} & a(1) K_{0}
\end{array}\right]_{-} } \\
& =\left(\operatorname{diag}_{i=1, \ldots, n} a\left(\frac{i}{n}\right) I_{2}\right)_{-} K_{n}^{[2]}(1)=\left(D_{n}\left(a I_{2}\right) T_{n}\left(f^{[2]}\right)\right)_{-},
\end{aligned}
$$

where $I_{2}$ is the $2 \times 2$ identity matrix and

$$
D_{n}\left(a I_{2}\right)=\operatorname{diag}_{i=1, \ldots, n} a\left(\frac{i}{n}\right) I_{2}=\left[\begin{array}{llll}
a\left(\frac{1}{n}\right) I_{2} & & & \\
& a\left(\frac{2}{n}\right) I_{2} & & \\
& & \ddots & \\
& & & a(1) I_{2}
\end{array}\right] .
$$

The sequence of matrices

$$
L_{n}^{[2]}(a)=D_{n}\left(a I_{2}\right) T_{n}\left(f^{[2]}\right)
$$

\footnotetext{
${ }^{2}$ In what follows, we use the notation $X_{-}$to indicate the matrix $X$ deprived of its last row and column.
} 


\section{ETNA}

Kent State University and

Johann Radon Institute (RICAM)

is an emblematic example of a block locally Toeplitz sequence. The singular values and eigenvalues of $L_{n}^{[2]}(a)$, exactly as those of $K_{n}^{[2]}(a)$, are nicely distributed according to

$$
\lim _{n \rightarrow \infty} \frac{1}{d_{n}} \sum_{i=1}^{d_{n}} F\left(\sigma_{i}\left(H_{n}\right)\right)=\frac{1}{2 \pi} \int_{0}^{1} \int_{-\pi}^{\pi} \frac{\sum_{i=1}^{s} F\left(\sigma_{i}\left(a(x) f^{[2]}(\theta)\right)\right)}{s} \mathrm{~d} \theta \mathrm{d} x
$$

and

$$
\lim _{n \rightarrow \infty} \frac{1}{d_{n}} \sum_{i=1}^{d_{n}} F\left(\lambda_{i}\left(H_{n}\right)\right)=\frac{1}{2 \pi} \int_{0}^{1} \int_{-\pi}^{\pi} \frac{\sum_{i=1}^{s} F\left(\lambda_{i}\left(a(x) f^{[2]}(\theta)\right)\right)}{s} \mathrm{~d} \theta \mathrm{d} x,
$$

where $H_{n}$ is either $L_{n}^{[2]}(a)$ or $K_{n}^{[2]}(a)$ and $d_{n}$ is either $2 n$ or $2 n-1$ depending on $H_{n}$. Considerations analogous to those reported above concerning the local (block) Toeplitz structure of $L_{n}^{[2]}(a)$ apply to this case as well. In particular, if we explore "locally" the matrix $L_{n}^{[2]}(a)$ using an ideal microscope and considering a large value of $n$, then we realize that the "local" structure of $L_{n}^{[2]}(a)$ is approximately the block Toeplitz structure generated by $f^{[2]}(\theta)$ and weighted through the function $a(x)$. The case $p>2$ is completely analogous to the case $p=2$ discussed here. We will come back to higher-order finite element discretizations of (1.5) in Section 6.2.

1.4. Contributions and structure of the present work. In the very recent works [55, 56], starting from the original intuition in [76, Section 3.3], the block version of the theory of GLT sequences-also known as the theory of block GLT sequences-has been developed in a systematic way as an extension of the theory of (scalar) GLT sequences [50, 51]. Such an extension is of the utmost importance in practical applications. In particular, it provides the necessary tools for computing the spectral distribution of block structured matrices arising from the discretization of systems of DEs [76, Section 3.3] and from the higher-order FE or discontinuous Galerkin (DG) approximation of scalar/vectorial DEs; see Section 1.3 and $[11,43,54,57]$. A few applications of the theory of block GLT sequences developed in $[55,56]$ have been presented in $[49,52]$.

It was soon noticed, however, that the theory of block GLT sequences in $[55,56]$ is not the most convenient extension of the theory of GLT sequences in [50, 51]. Indeed, the presentation in $[55,56]$ is unnecessarily complicated and, moreover, it is also incomplete because several results from $[50,51]$ have been ignored. In addition, many important theoretical advances obtained in recent works $[3,4,5,6,7,8]$ have not been generalized to the block case. The purpose of the present work is twofold.

- Firstly, we review, refine, and considerably extend the papers $[55,56]$ by presenting in a systematic way the most convenient and complete version of the theory of block GLT sequences, that is, the correct generalization to the block case of the theory of GLT sequences covered in $[50,51]$. We also extend to the block case several important results from $[3,4,5,6,7,8]$, which allow us to both simplify the presentation and make it more elegant with respect to all previous works $[50,51,55,56,75,76]$.

- Secondly, we present several emblematic applications of the theory of block GLT sequences in the context of DE discretizations, including (but not limited to) those already addressed in $[49,52]$.

The present work is structured as a long research article in book form. Chapter 2 collects the necessary preliminaries. Chapters 3 and 4 cover the theory of block GLT sequences, which is finally summarized in Chapter 5. Chapter 6 is devoted to applications. 


\section{ETNA}

Kent State University and

Johann Radon Institute (RICAM)

2. Mathematical background. This chapter collects the necessary preliminaries for developing the theory of block GLT sequences.

\subsection{Notation and terminology.}

- $O_{m}$ and $I_{m}$ denote, respectively, the $m \times m$ zero matrix and the $m \times m$ identity matrix. Sometimes, when the size $m$ can be inferred from the context, $O$ and $I$ are used instead of $O_{m}$ and $I_{m}$. The symbol $O$ is also used to indicate rectangular zero matrices whose sizes are clear from the context.

- For every $s \in \mathbb{N}$ and every $\alpha, \beta=1, \ldots, s$, we denote by $E_{\alpha \beta}^{(s)}$ the $s \times s$ matrix having 1 in position $(\alpha, \beta)$ and 0 elsewhere.

- For every $s, n \in \mathbb{N}$, we denote by $\Pi_{n, s}$ the permutation matrix given by

$$
\Pi_{n, s}=\left[\begin{array}{c}
I_{s} \otimes \mathbf{e}_{1}^{T} \\
I_{s} \otimes \mathbf{e}_{2}^{T} \\
\vdots \\
I_{s} \otimes \mathbf{e}_{n}^{T}
\end{array}\right]=\sum_{k=1}^{n} \mathbf{e}_{k} \otimes I_{s} \otimes \mathbf{e}_{k}^{T},
$$

where $\otimes$ denotes the tensor (Kronecker) product (see Section 2.2.2) and $\mathbf{e}_{1}, \ldots, \mathbf{e}_{n}$ are the vectors of the canonical basis of $\mathbb{C}^{n}$. For every $s, r, n \in \mathbb{N}$, we define the permutation matrix

$$
\Pi_{n, s, r}=\Pi_{n, s} \otimes I_{r} .
$$

- The eigenvalues and the singular values of a matrix $X \in \mathbb{C}^{m \times m}$ are denoted by $\lambda_{j}(X)$, $j=1, \ldots, m$, and $\sigma_{j}(X), j=1, \ldots, m$, respectively. The maximum and minimum singular values of $X$ are also denoted by $\sigma_{\max }(X)$ and $\sigma_{\min }(X)$, respectively. The spectrum of $X$ is denoted by $\Lambda(X)$.

- If $1 \leq p \leq \infty$, the symbol $|\cdot|_{p}$ denotes both the $p$-norm of vectors and the associated operator norm for matrices:

$$
\begin{aligned}
|\mathbf{x}|_{p} & = \begin{cases}\left(\sum_{i=1}^{m}\left|x_{i}\right|^{p}\right)^{1 / p}, & \text { if } 1 \leq p<\infty, \\
\max _{i=1, \ldots, m}\left|x_{i}\right|, & \text { if } p=\infty,\end{cases} \\
|X|_{p} & =\max _{\substack{\mathbf{x} \in \mathbb{C}^{m} \\
\mathbf{x} \neq \mathbf{0}}} \frac{|X \mathbf{x}|_{p}}{|\mathbf{x}|_{p}}, \quad X \in \mathbb{C}^{m},
\end{aligned}
$$

The 2-norm $|\cdot|_{2}$ is also known as the spectral (or Euclidean) norm; it will be preferably denoted by $\|\cdot\|$.

- Given $X \in \mathbb{C}^{m \times m}$ and $1 \leq p \leq \infty,\|X\|_{p}$ denotes the Schatten $p$-norm of $X$, which is defined as the $p$-norm of the vector $\left(\sigma_{1}(X), \ldots, \sigma_{m}(X)\right)$. The Schatten 1-norm is also called the trace-norm. The Schatten 2-norm $\|X\|_{2}$ coincides with the classical Frobenius $\operatorname{norm}\left(\sum_{i, j=1}^{m}\left|x_{i j}\right|^{2}\right)^{1 / 2}$. The Schatten $\infty$-norm $\|X\|_{\infty}=\sigma_{\max }(X)$ is the classical 2-norm $\|X\|$. For more on Schatten $p$-norms, see [13].

- $\Re(X)$ and $\Im(X)$ are, respectively, the real and imaginary parts of the (square) matrix $X$, i.e., $\Re(X)=\frac{X+X^{*}}{2}$ and $\Im(X)=\frac{X-X^{*}}{2 \mathrm{i}}$, where $X^{*}$ is the conjugate transpose of $X$ and $\mathrm{i}$ is the imaginary unit.

- If $X \in \mathbb{C}^{m \times m}$, we denote by $X^{\dagger}$ the Moore-Penrose pseudoinverse of $X$.

- $C_{c}(\mathbb{C})\left(\right.$ resp., $\left.C_{c}(\mathbb{R})\right)$ is the space of complex-valued continuous functions defined on $\mathbb{C}$ $($ resp., $\mathbb{R})$ and with bounded support. 


\section{ETNA}

Kent State University and

Johann Radon Institute (RICAM)

- If $z \in \mathbb{C}$ and $\varepsilon>0$, we denote by $D(z, \varepsilon)$ the open disk with center $z$ and radius $\varepsilon$, i.e., $D(z, \varepsilon)=\{w \in \mathbb{C}:|w-z|<\varepsilon\}$. If $S \subseteq \mathbb{C}$ and $\varepsilon>0$, we denote by $D(S, \varepsilon)$ the $\varepsilon$-expansion of $S$, which is defined as $D(S, \varepsilon)=\bigcup_{z \in S} D(z, \varepsilon)$.

- $\chi_{E}$ is the characteristic (indicator) function of the set $E$.

- A concave bounded continuous function $\varphi:[0, \infty) \rightarrow[0, \infty)$ such that $\varphi(0)=0$ and $\varphi>0$ on $(0, \infty)$ is referred to as a gauge function. It can be shown that any gauge function $\varphi$ is non-decreasing and subadditive, i.e., $\varphi(x+y) \leq \varphi(x)+\varphi(y)$ for all $x, y \in[0, \infty)$; see, e.g., [50, Exercise 2.4].

- If $g: D \rightarrow \mathbb{C}$ is continuous over $D$, with $D \subseteq \mathbb{C}^{k}$ for some $k$, we denote by $\omega_{g}(\cdot)$ the modulus of continuity of $g$,

$$
\omega_{g}(\delta)=\sup _{\substack{\mathbf{x}, \mathbf{y} \in D \\|\mathbf{x}-\mathbf{y}|_{\infty} \leq \delta}}|g(\mathbf{x})-g(\mathbf{y})|, \quad \delta>0 .
$$

- A matrix-valued function $a:[0,1] \rightarrow \mathbb{C}^{r \times r}$ is said to be Riemann-integrable if its components $a_{\alpha \beta}:[0,1] \rightarrow \mathbb{C}, \alpha, \beta=1, \ldots, r$, are Riemann-integrable. We remark that a complex-valued function $g$ is Riemann-integrable when its real and imaginary parts $\Re(g)$ and $\Im(g)$ are Riemann-integrable in the classical sense.

- $\mu_{k}$ denotes the Lebesgue measure in $\mathbb{R}^{k}$. Throughout this work, unless stated otherwise, all the terminology from measure theory (such as "measurable set", "measurable function", "a.e.", etc.) is always referred to the Lebesgue measure.

- Let $D \subseteq \mathbb{R}^{k}$, let $r \geq 1$, and $1 \leq p \leq \infty$. A matrix-valued function $f: D \rightarrow \mathbb{C}^{r \times r}$ is said to be measurable (resp., continuous, a.e. continuous, bounded, in $L^{p}(D)$, in $C^{\infty}(D)$, etc.) if its components $f_{\alpha \beta}: D \rightarrow \mathbb{C}, \alpha, \beta=1, \ldots, r$, are measurable (resp., continuous, a.e. continuous, bounded, in $L^{p}(D)$, in $C^{\infty}(D)$, etc.). The space of functions $f: D \rightarrow \mathbb{C}^{r \times r}$ belonging to $L^{p}(D)$ will be denoted by $L^{p}(D, r)$ in order to emphasize the dependence on $r$. For the space of scalar functions $L^{p}(D, 1)$, we will preferably use the traditional simpler notation $L^{p}(D)$.

- Let $f_{m}, f: D \subseteq \mathbb{R}^{k} \rightarrow \mathbb{C}^{r \times r}$ be measurable. We say that $f_{m}$ converges to $f$ in measure (resp., a.e., in $L^{p}(D)$, etc.) if $\left(f_{m}\right)_{\alpha \beta}$ converges to $f_{\alpha \beta}$ in measure (resp., a.e., in $L^{p}(D)$, etc.) for all $\alpha, \beta=1, \ldots, r$.

- If $D$ is any measurable subset of some $\mathbb{R}^{k}$ and $r \in \mathbb{N}$, we set

$$
\mathfrak{M}_{D}^{(r)}=\left\{f: D \rightarrow \mathbb{C}^{r \times r}: f \text { is measurable }\right\} .
$$

If $D=[0,1] \times[-\pi, \pi]$, we preferably use the notation $\mathfrak{M}^{(r)}$ instead of $\mathfrak{M}_{D}^{(r)}$ :

$$
\mathfrak{M}^{(r)}=\left\{\kappa:[0,1] \times[-\pi, \pi] \rightarrow \mathbb{C}^{r \times r}: \kappa \text { is measurable }\right\} .
$$

- We use a notation borrowed from probability theory to indicate sets. For example, if $f, g: D \subseteq \mathbb{R}^{k} \rightarrow \mathbb{C}^{r \times r}$, then $\left\{\sigma_{\max }(f)>0\right\}=\left\{\mathbf{x} \in D: \sigma_{\max }(f(\mathbf{x}))>0\right\}$, $\mu_{k}\{\|f-g\| \geq \varepsilon\}$ is the measure of the set $\{\mathbf{x} \in D:\|f(\mathbf{x})-g(\mathbf{x})\| \geq \varepsilon\}$, etc.

- A function of the form $f(\theta)=\sum_{j=-q}^{q} f_{j} e^{\mathrm{i} j \theta}$ with $f_{-q}, \ldots, f_{q} \in \mathbb{C}^{r \times r}$ is said to be a $\left(r \times r\right.$ matrix-valued) trigonometric polynomial. If $f_{-q} \neq O_{r}$ or $f_{q} \neq O_{r}$, then the number $q$ is referred to as the degree of $f$.

- A sequence of matrices is a sequence of the form $\left\{A_{n}\right\}_{n}$, where $A_{n}$ is a square matrix of size $d_{n}$ such that $d_{n} \rightarrow \infty$ as $n \rightarrow \infty$.

- Given $s \in \mathbb{N}$, an $s$-block matrix-sequence is a special sequence of matrices of the form $\left\{A_{n}\right\}_{n}$, where $A_{n}$ is a square matrix of size $d_{n}=s n$. 


\subsection{Preliminaries on matrix analysis.}

2.2.1. Matrix norms. For the reader's convenience, we report in this section some matrix-norm inequalities that we shall use throughout this work. Given a matrix $X \in \mathbb{C}^{m \times m}$, important bounds for the 2-norm $\|X\|$ in terms of the components of $X$ are the following [50, pp. 29-30]:

$$
\begin{aligned}
& \left|x_{i j}\right| \leq\|X\|, \quad i, j=1, \ldots, m, \quad X \in \mathbb{C}^{m \times m}, \\
& \|X\| \leq \sqrt{|X|_{1}|X|_{\infty}} \leq \max \left(|X|_{1},|X|_{\infty}\right) \leq \sum_{i, j=1}^{m}\left|x_{i j}\right|, \quad X \in \mathbb{C}^{m \times m} .
\end{aligned}
$$

Since $\|X\|=\sigma_{\max }(X)$ and $\operatorname{rank}(X)$ is the number of nonzero singular values of $X$, we have

$$
\|X\| \leq\|X\|_{1} \leq \operatorname{rank}(X)\|X\| \leq m\|X\|, \quad X \in \mathbb{C}^{m \times m} .
$$

Another important trace-norm inequality is the following [50, p. 33]:

$$
\|X\|_{1} \leq \sum_{i, j=1}^{m}\left|x_{i j}\right|, \quad X \in \mathbb{C}^{m \times m} .
$$

The last inequality provides a bound for the Frobenius norm in terms of the spectral norm and the trace-norm:

$$
\|X\|_{2}=\sqrt{\sum_{i=1}^{m} \sigma_{i}(X)^{2}} \leq \sqrt{\sigma_{\max }(X) \sum_{i=1}^{m} \sigma_{i}(X)}=\sqrt{\|X\|\|X\|_{1}}, \quad X \in \mathbb{C}^{m \times m} .
$$

2.2.2. Tensor products and direct sums. If $X, Y$ are matrices of any dimension, say $X \in \mathbb{C}^{m_{1} \times m_{2}}$ and $Y \in \mathbb{C}^{\ell_{1} \times \ell_{2}}$, then the tensor (Kronecker) product of $X$ and $Y$ is the $m_{1} \ell_{1} \times m_{2} \ell_{2}$ matrix defined by

$$
X \otimes Y=\left[x_{i j} Y\right]_{\substack{i=1, \ldots, m_{1} \\
j=1, \ldots, m_{2}}}=\left[\begin{array}{ccc}
x_{11} Y & \cdots & x_{1 m_{2}} Y \\
\vdots & & \vdots \\
x_{m_{1} 1} Y & \cdots & x_{m_{1} m_{2}} Y
\end{array}\right]
$$

and the direct sum of $X$ and $Y$ is the $\left(m_{1}+\ell_{1}\right) \times\left(m_{2}+\ell_{2}\right)$ matrix defined by

$$
X \oplus Y=\operatorname{diag}(X, Y)=\left[\begin{array}{cc}
X & O \\
O & Y
\end{array}\right] .
$$

Tensor products and direct sums possess a lot of nice algebraic properties.

(i) Associativity: for all matrices $X, Y, Z$,

$$
\begin{aligned}
& (X \otimes Y) \otimes Z=X \otimes(Y \otimes Z), \\
& (X \oplus Y) \oplus Z=X \oplus(Y \oplus Z) .
\end{aligned}
$$

(ii) If $X_{1}, X_{2}$ can be multiplied and $Y_{1}, Y_{2}$ can be multiplied, then

$$
\begin{aligned}
& \left(X_{1} \otimes Y_{1}\right)\left(X_{2} \otimes Y_{2}\right)=\left(X_{1} X_{2}\right) \otimes\left(Y_{1} Y_{2}\right), \\
& \left(X_{1} \oplus Y_{1}\right)\left(X_{2} \oplus Y_{2}\right)=\left(X_{1} X_{2}\right) \oplus\left(Y_{1} Y_{2}\right) .
\end{aligned}
$$


(iii) For all matrices $X, Y$,

$$
\begin{aligned}
& (X \otimes Y)^{*}=X^{*} \otimes Y^{*}, \quad(X \otimes Y)^{T}=X^{T} \otimes Y^{T} \\
& (X \oplus Y)^{*}=X^{*} \oplus Y^{*}, \quad(X \oplus Y)^{T}=X^{T} \oplus Y^{T} .
\end{aligned}
$$

(iv) Bilinearity (of tensor products): for each fixed matrix $X$, the application

$$
Y \mapsto X \otimes Y
$$

is linear on $\mathbb{C}^{\ell_{1} \times \ell_{2}}$ for all $\ell_{1}, \ell_{2} \in \mathbb{N}$; for each fixed matrix $Y$, the application

$$
X \mapsto X \otimes Y
$$

is linear on $\mathbb{C}^{m_{1} \times m_{2}}$ for all $m_{1}, m_{2} \in \mathbb{N}$.

From (i)-(iv), a lot of other properties follow. For example, if $\mathbf{v}$ is a column vector and $X, Y$ are matrices that can be multiplied, then $(\mathbf{v} \otimes X) Y=(\mathbf{v} \otimes X)([1] \otimes Y)=\mathbf{v} \otimes(X Y)$. If $X, Y$ are invertible, then $X \otimes Y$ is invertible with inverse $X^{-1} \otimes Y^{-1}$. If $X, Y$ are normal (resp., Hermitian, symmetric, unitary), then $X \otimes Y$ is also normal (resp., Hermitian, symmetric, unitary). If $X \in \mathbb{C}^{m \times m}$ and $Y \in \mathbb{C}^{\ell \times \ell}$, then the eigenvalues and singular values of $X \otimes Y$ are given by

$$
\begin{aligned}
& \left\{\lambda_{i}(X) \lambda_{j}(Y): i=1, \ldots, m, j=1, \ldots, \ell\right\}, \\
& \left\{\sigma_{i}(X) \sigma_{j}(Y): i=1, \ldots, m, j=1, \ldots, \ell\right\}
\end{aligned}
$$

and the eigenvalues and singular values of $X \oplus Y$ are given by

$$
\begin{aligned}
& \left\{\lambda_{i}(X), \lambda_{j}(Y): i=1, \ldots, m, j=1, \ldots, \ell\right\}, \\
& \left\{\sigma_{i}(X), \sigma_{j}(Y): i=1, \ldots, m, j=1, \ldots, \ell\right\} ;
\end{aligned}
$$

see [50, Exercise 2.5]. In particular, for all $X \in \mathbb{C}^{m \times m}, Y \in \mathbb{C}^{\ell \times \ell}$, and $1 \leq p \leq \infty$, we have

$$
\begin{aligned}
& \|X \otimes Y\|_{p}=\|X\|_{p}\|Y\|_{p}, \\
& \|X \oplus Y\|_{p}=\left|\left(\|X\|_{p},\|Y\|_{p}\right)\right|_{p}= \begin{cases}\left(\|X\|_{p}^{p}+\|Y\|_{p}^{p}\right)^{1 / p}, & \text { if } 1 \leq p<\infty, \\
\max \left(\|X\|_{\infty},\|Y\|_{\infty}\right), & \text { if } p=\infty .\end{cases}
\end{aligned}
$$

\subsection{Preliminaries on measure and integration theory.}

2.3.1. Measurability. The following lemma is derived from the results in $[13, \mathrm{Sec}-$ tion VI.1]. It will be used essentially everywhere in this work, either explicitly or implicitly.

LEMma 2.1. Let $f: D \subseteq \mathbb{R}^{k} \rightarrow \mathbb{C}^{r \times r}$ be measurable and $g: \mathbb{C}^{r} \rightarrow \mathbb{C}$ be continuous and symmetric in its $r$ arguments, i.e., $g\left(\lambda_{1}, \ldots, \lambda_{r}\right)=g\left(\lambda_{\rho(1)}, \ldots, \lambda_{\rho(r)}\right)$ for all permutations $\rho$ of $\{1, \ldots, r\}$. Then, the function $\mathbf{x} \mapsto g\left(\lambda_{1}(f(\mathbf{x})), \ldots, \lambda_{r}(f(\mathbf{x}))\right)$ is well-defined (independently of the ordering of the eigenvalues of $f(\mathbf{x}))$ and measurable. As a consequence:

- the function $\mathbf{x} \mapsto g\left(\sigma_{1}(f(\mathbf{x})), \ldots, \sigma_{r}(f(\mathbf{x}))\right)$ is measurable;

- the functions $\mathbf{x} \mapsto \sum_{i=1}^{r} F\left(\lambda_{i}(f(\mathbf{x}))\right)$ and $\mathbf{x} \mapsto \sum_{i=1}^{r} F\left(\sigma_{i}(f(\mathbf{x}))\right)$ are measurable for all continuous $F: \mathbb{C} \rightarrow \mathbb{C}$;

- the function $\mathbf{x} \mapsto\|f(\mathbf{x})\|_{p}$ is measurable for all $p \in[1, \infty]$.

REMARK 2.2 (Existence of an ordering for the eigenvalues $\lambda_{i}(f(\mathrm{x}))$ ). Let the function $f: D \subseteq \mathbb{R}^{k} \rightarrow \mathbb{C}^{r \times r}$ be measurable. In the case where all the eigenvalues of the matrix $f(\mathbf{x})$ are real for almost every $\mathbf{x} \in D$, one can define the eigenvalue function $\lambda_{i}(f(\mathbf{x}))$ as a measurable function taking the value of the $i$ th largest eigenvalue of $f(\mathbf{x})$. In general, even if 
$f$ is continuous, we are not able to find $r$ continuous functions acting as eigenvalue functions; see [13, Example VI.1.3]. Thus, a convenient ordering on the eigenvalues $\lambda_{i}(f(\mathbf{x}))$ cannot be prescribed beforehand. In such cases, $\lambda_{i}(f(\mathbf{x}))$ has not to be intended as a function in $\mathbf{x}$ but as an element of the spectrum $\Lambda(f(\mathbf{x}))$ ordered in an arbitrary way. Lemma 2.1 is then important as it allows us to work with the spectrum as a whole, without having to specify which ordering we are imposing on the eigenvalues $\lambda_{i}(f(\mathbf{x}))$. In what follows, when we talk about the $i$ th eigenvalue function $\lambda_{i}(f(\mathbf{x}))$, we are implicitly assuming that this function exists as a measurable function; more precisely, we are assuming that there exist $r$ measurable functions $\lambda_{i}(f(\mathbf{x})), i=1, \ldots, r$, from $D$ to $\mathbb{C}$ such that, for each fixed $\mathbf{x} \in D$, the eigenvalues of $f(\mathbf{x})$ are given by $\lambda_{1}(f(\mathbf{x})), \ldots, \lambda_{r}(f(\mathbf{x}))$.

2.3.2. Essential range of matrix-valued functions. If $f: D \subseteq \mathbb{R}^{k} \rightarrow \mathbb{C}^{r \times r}$ is a measurable matrix-valued function, then the essential range of $f$ is denoted by $\mathcal{E} \mathcal{R}(f)$ and is defined as follows:

$$
\begin{aligned}
\mathcal{E R}(f) & =\left\{z \in \mathbb{C}: \mu_{k}\left\{\exists j \in\{1, \ldots, r\}: \lambda_{j}(f) \in D(z, \varepsilon)\right\}>0 \text { for all } \varepsilon>0\right\} \\
& =\left\{z \in \mathbb{C}: \mu_{k}\left\{\min _{j=1, \ldots, r}\left|\lambda_{j}(f)-z\right|<\varepsilon\right\}>0 \text { for all } \varepsilon>0\right\} .
\end{aligned}
$$

Note that $\mathcal{E} \mathcal{R}(f)$ is well-defined because the function $\mathbf{x} \mapsto \min _{j=1, \ldots, r}\left|\lambda_{j}(f(\mathbf{x}))-z\right|$ is measurable by Lemma 2.1. In the case where the eigenvalue functions $\lambda_{j}(f): D \rightarrow \mathbb{C}$, $j=1, \ldots, r$, are measurable, we have

$$
\mathcal{E} \mathcal{R}(f)=\bigcup_{j=1}^{r} \mathcal{E} \mathcal{R}\left(\lambda_{j}(f)\right)
$$

LEMMA 2.3. Let $f: D \subseteq \mathbb{R}^{k} \rightarrow \mathbb{C}^{r \times r}$ be measurable. Then $\mathcal{E} \mathcal{R}(f)$ is closed and $\Lambda(f) \subseteq \mathcal{E} \mathcal{R}(f)$ a.e.

Proof. We show that the complement of $\mathcal{E} \mathcal{R}(f)$ is open. If $z \in \mathbb{C} \backslash \mathcal{E} \mathcal{R}(f)$, then $\mu_{k}\left\{\exists j \in\{1, \ldots, r\}: \lambda_{j}(f) \in D(z, \varepsilon)\right\}=0$ for some $\varepsilon>0$. Each point $w \in D(z, \varepsilon)$ has a neighborhood $D(w, \delta)$ such that $D(w, \delta) \subseteq D(z, \varepsilon)$, and consequently it follows that $\mu_{k}\left\{\exists j \in\{1, \ldots, r\}: \lambda_{j}(f) \in D(w, \delta)\right\}=0$. We conclude that $D(z, \varepsilon) \subseteq \mathbb{C} \backslash \mathcal{E R}(f)$, hence $\mathbb{C} \backslash \mathcal{E} \mathcal{R}(f)$ is open.

To prove that $\Lambda(f) \subseteq \mathcal{E R}(f)$ a.e., let

$$
\mathcal{B}=\left\{D\left(q, \frac{1}{m}\right): q=a+\mathrm{i} b, \quad a, b \in \mathbb{Q}, \quad m \in \mathbb{N}\right\}
$$

$\mathcal{B}$ is a topological basis of $\mathbb{C}$, i.e., for each open set $U \subseteq \mathbb{C}$ and each $u \in U$, there exists an element of $\mathcal{B}$ which contains $u$ and is contained in $U$. Since $\mathbb{C} \backslash \mathcal{E} \mathcal{R}(f)$ is open and every $z \in \mathbb{C} \backslash \mathcal{E} \mathcal{R}(f)$ has a neighborhood $D(z, \varepsilon)$ such that

$$
\mu_{k}\left\{\exists j \in\{1, \ldots, r\}: \lambda_{j}(f) \in D(z, \varepsilon)\right\}=0
$$

(by definition of $\mathcal{E} \mathcal{R}(f)$ ), for each $z \in \mathbb{C} \backslash \mathcal{E R}(f)$ there exists an element of $\mathcal{B}$, say $D_{z}=D\left(q_{z}, \frac{1}{m_{z}}\right)$, such that $z \in D_{z} \subseteq \mathbb{C} \backslash \mathcal{E R}(f)$ and

$$
\mu_{k}\left\{\exists j \in\{1, \ldots, r\}: \lambda_{j}(f) \in D_{z}\right\}=0
$$


Let $\mathcal{C}$ be the subset of $\mathcal{B}$ given by $\mathcal{C}=\left\{D_{z}: z \in \mathbb{C} \backslash \mathcal{E R}(f)\right\}$. Since $\mathcal{B}$ is countable, $\mathcal{C}$ is countable as well, say $\mathcal{C}=\left\{C_{\ell}: \ell=1,2, \ldots\right\}$, and we have

$$
\begin{aligned}
\{\Lambda(f) \not \subset \mathcal{E R}(f)\} & =\bigcup_{z \in \mathbb{C} \backslash \mathcal{E R}(f)}\left\{\exists j \in\{1, \ldots, r\}: \lambda_{j}(f)=z\right\} \\
& \subseteq \bigcup_{z \in \mathbb{C} \backslash \mathcal{E R}(f)}\left\{\exists j \in\{1, \ldots, r\}: \lambda_{j}(f) \in D_{z}\right\} \\
& =\bigcup_{\ell=1}^{\infty}\left\{\exists j \in\{1, \ldots, r\}: \lambda_{j}(f) \in C_{\ell}\right\},
\end{aligned}
$$

which completes the proof because the last set is a countable union of sets having zero measure, and so it has zero measure as well.

2.3.3. $L^{p}$-norms of matrix-valued functions. Let $D$ be any measurable subset of some $\mathbb{R}^{k}$, let $r \geq 1$, and let $1 \leq p \leq \infty$. For any measurable function $f: D \rightarrow \mathbb{C}^{r \times r}$ we define

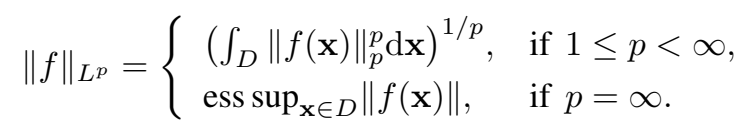

Note that this definition is well-posed by Lemma 2.1. In the case where $r=1$, it reduces to the classical definition of $L^{p}$-norms for scalar functions. As highlighted in [38, p. 164], for every $p \in[1, \infty]$, there exist constants $A_{p}, B_{p}>0$ such that, for all $f \in L^{p}(D, r)$,

$$
\begin{array}{cl}
A_{p}\|f\|_{L^{p}}^{p} \leq \sum_{\alpha, \beta=1}^{r}\left\|f_{\alpha \beta}\right\|_{L^{p}}^{p} \leq B_{p}\|f\|_{L^{p}}^{p}, & \text { if } 1 \leq p<\infty, \\
A_{\infty}\|f\|_{L^{\infty}} \leq \max _{\alpha, \beta=1, \ldots, r}\left\|f_{\alpha \beta}\right\|_{L^{\infty}} \leq B_{\infty}\|f\|_{L^{\infty}}, & \text { if } p=\infty .
\end{array}
$$

This means that $L^{p}(D, r)$, which we have defined in Section 2.1 as the set of functions $f: D \rightarrow \mathbb{C}^{r \times r}$ such that each component $f_{\alpha \beta}$ belongs to $L^{p}(D)$, can also be defined as the set of measurable functions $f: D \rightarrow \mathbb{C}^{r \times r}$ such that $\|f\|_{L^{p}}<\infty$. Moreover, if we identify two functions $f, g \in L^{p}(D, r)$ whenever $f(\mathbf{x})=g(\mathbf{x})$ for almost every $\mathbf{x} \in D$, then the map $f \mapsto\|f\|_{L^{p}}$ is a norm on $L^{p}(D, r)$, which induces on $L^{p}(D, r)$ the componentwise $L^{p}$ convergence, that is, $f_{m} \rightarrow f$ in $L^{p}(D, r)$ according to the norm $\|\cdot\|_{L^{p}}$ if and only if $\left(f_{m}\right)_{\alpha \beta} \rightarrow f_{\alpha \beta}$ in $L^{p}(D)$ for all $\alpha, \beta=1, \ldots, r$.

2.3.4. Convergence in measure and the topology $\tau_{\text {measure }}$. The convergence in measure plays a central role in the theory of block GLT sequences. A basic lemma about this convergence is reported below [16, Corollary 2.2.6].

LEMMA 2.4. Let $f_{m}, g_{m}, f, g: D \subseteq \mathbb{R}^{k} \rightarrow \mathbb{C}^{r \times r}$ be measurable functions.

- If $f_{m} \rightarrow f$ in measure and $g_{m} \rightarrow g$ in measure, then $\alpha f_{m}+\beta g_{m} \rightarrow \alpha f+\beta g$ in measure for all $\alpha, \beta \in \mathbb{C}$.

- If $f_{m} \rightarrow f$ in measure, $g_{m} \rightarrow g$ in measure, and $\mu_{k}(D)<\infty$, then $f_{m} g_{m} \rightarrow f g$ in measure.

Let $\varphi:[0, \infty) \rightarrow[0, \infty)$ be a gauge function, let $D \subset \mathbb{R}^{k}$ be a measurable set with $0<\mu_{k}(D)<\infty$, and let

$$
\mathfrak{M}_{D}^{(r)}=\left\{f: D \rightarrow \mathbb{C}^{r \times r}: f \text { is measurable }\right\} .
$$


Suppose first that $r=1$. If we define

$$
\begin{aligned}
p_{\text {measure }}^{\varphi}(f) & =\frac{1}{\mu_{k}(D)} \int_{D} \varphi(|f|), \quad f \in \mathfrak{M}_{D}^{(1)}, \\
d_{\text {measure }}^{\varphi}(f, g) & =p_{\text {measure }}^{\varphi}(f-g), \quad f, g \in \mathfrak{M}_{D}^{(1)},
\end{aligned}
$$

then $d_{\text {measure }}^{\varphi}$ is a complete pseudometric on $\mathfrak{M}_{D}^{(1)}$ such that a sequence $\left\{f_{m}\right\}_{m} \subset \mathfrak{M}_{D}^{(1)}$ converges to $f \in \mathfrak{M}_{D}^{(1)}$ according to $d_{\text {measure }}^{\varphi}$ if and only if $f_{m} \rightarrow f$ in measure. In particular, $d_{\text {measure }}^{\varphi}(f, g)=0$ if and only if $f \rightarrow g$ in measure, that is, if and only if $f=g$ a.e. The topology induced on $\mathfrak{M}_{D}^{(1)}$ by $d_{\text {measure }}^{\varphi}$ is the same for all gauge functions $\varphi$; it is denoted by $\tau_{\text {measure }}$ and is referred to as the topology of convergence in measure on $\mathfrak{M}_{D}^{(1)}$.

Suppose now that $r \geq 1$. If we define

$$
\begin{aligned}
\hat{p}_{\text {measure }}^{\varphi}(f) & =\max _{\alpha, \beta=1, \ldots, r} p_{\text {measure }}^{\varphi}\left(f_{\alpha \beta}\right), & f \in \mathfrak{M}_{D}^{(r)}, \\
\hat{d}_{\text {measure }}^{\varphi}(f, g) & =\hat{p}_{\text {measure }}^{\varphi}(f-g), & f, g \in \mathfrak{M}_{D}^{(r)},
\end{aligned}
$$

then $\hat{d}_{\text {measure }}^{\varphi}$ is a complete pseudometric on $\mathfrak{M}_{D}^{(r)}$ such that a sequence $\left\{f_{m}\right\}_{m} \subset \mathfrak{M}_{D}^{(r)}$ converges to $f \in \mathfrak{M}_{D}^{(r)}$ according to $\hat{d}_{\text {measure }}^{\varphi}$ if and only if $f_{m} \rightarrow f$ in measure. In particular, $\hat{d}_{\text {measure }}^{\varphi}(f, g)=0$ if and only if $f \rightarrow g$ in measure, that is, if and only if $f=g$ a.e. The topology induced on $\mathfrak{M}_{D}^{(r)}$ by $\hat{d}_{\text {measure }}^{\varphi}$ is the same for all gauge functions $\varphi$; it is denoted by $\tau_{\text {measure }}$ and is referred to as the topology of convergence in measure on $\mathfrak{M}_{D}^{(r)}$.

Now, let

$$
\begin{aligned}
p_{\text {measure }}^{\varphi}(f) & =\frac{1}{\mu_{k}(D)} \int_{D} \frac{\sum_{i=1}^{r} \varphi\left(\sigma_{i}(f)\right)}{r}, & f \in \mathfrak{M}_{D}^{(r)}, \\
d_{\text {measure }}^{\varphi}(f, g) & =p_{\text {measure }}^{\varphi}(f-g), & f, g \in \mathfrak{M}_{D}^{(r)} .
\end{aligned}
$$

By using the Rotfel'd theorem [13, Theorem IV.2.14], it is not difficult to see that $d_{\text {measure }}^{\varphi}$ is another pseudometric on $\mathfrak{M}_{D}^{(r)}$, which is also metrically equivalent to $\hat{d}_{\text {measure. Indeed, taking }}^{\varphi}$ into account that $\|f\|=\sigma_{\max }(f)$, by (2.2), (2.3), the subadditivity and the monotonicity of $\varphi$, we have

$$
\begin{aligned}
\hat{p}_{\text {measure }}^{\varphi}(f) & =\max _{\alpha, \beta=1, \ldots, r} p_{\text {measure }}\left(f_{\alpha \beta}\right)=\max _{\alpha, \beta=1, \ldots, r} \frac{1}{\mu_{k}(D)} \int_{D} \varphi\left(\left|f_{\alpha \beta}\right|\right) \\
& \leq \frac{1}{\mu_{k}(D)} \int_{D} \varphi(\|f\|) \leq r p_{\text {measure }}^{\varphi}(f), \\
p_{\text {measure }}^{\varphi}(f) & =\frac{1}{\mu_{k}(D)} \int_{D} \frac{\sum_{i=1}^{r} \varphi\left(\sigma_{i}(f)\right)}{r} \leq \frac{1}{\mu_{k}(D)} \int_{D} \varphi(\|f\|) \\
& \leq \frac{1}{\mu_{k}(D)} \int_{D} \varphi\left(\sum_{\alpha, \beta=1}^{r}\left|f_{\alpha \beta}\right|\right) \leq \frac{1}{\mu_{k}(D)} \int_{D} \sum_{\alpha, \beta=1}^{r} \varphi\left(\left|f_{\alpha \beta}\right|\right) \\
& \leq r^{2} \max _{\alpha, \beta=1, \ldots, r} p_{\text {measure }}^{\varphi}\left(f_{\alpha \beta}\right)=r^{2} \hat{p}_{\text {measure }}^{\varphi}(f) .
\end{aligned}
$$

In particular, $d_{\text {measure }}^{\varphi}$ induces on $\mathfrak{M}_{D}^{(r)}$ the topology $\tau_{\text {measure }}$ of convergence in measure and it is complete on $\mathfrak{M}_{D}^{(r)}$, just as $\hat{d}_{\text {measure. Throughout this work, we will use the notations }}^{\varphi}$

$$
p_{\text {measure }}=p_{\text {measure }}^{\psi}, \quad d_{\text {measure }}=d_{\text {measure }}^{\psi}, \quad \psi(x)=\frac{x}{1+x} .
$$




\section{ETNA}

Kent State University and

Johann Radon Institute (RICAM)

Lemma 2.5. Let $g_{m}, g: D \subset \mathbb{R}^{k} \rightarrow \mathbb{C}^{r \times r}$ be measurable functions defined on a set $D$ with $0<\mu_{k}(D)<\infty$. If

$$
\lim _{m \rightarrow \infty} \frac{1}{\mu_{k}(D)} \int_{D} \frac{\sum_{j=1}^{r} F\left(\sigma_{j}\left(g_{m}-g\right)\right)}{r}=F(0), \quad \forall F \in C_{c}(\mathbb{R}),
$$

then $g_{m} \rightarrow g$ in measure.

Proof. Suppose by contradiction that $g_{m} \not \supset g$ in measure. Then, there exist $\varepsilon, \delta>0$ and a subsequence $\left\{g_{m_{i}}\right\}_{i}$ such that, for all $i$,

$$
\mu_{k}\left\{\left\|g_{m_{i}}-g\right\| \geq \varepsilon\right\} \geq \delta .
$$

Take a real function $F \in C_{c}(\mathbb{R})$ such that $F(0)=1=\max _{y \in \mathbb{R}} F(y)$ and $F(y)=$ 0 over $\{y \in \mathbb{R}:|y| \geq \varepsilon\}$. By the previous inequality and taking into account that $\left\|g_{m_{i}}-g\right\|=\sigma_{\max }\left(g_{m_{i}}-g\right)$, for all $i$, we have

$$
\begin{aligned}
& \frac{1}{\mu_{k}(D)} \int_{D} \frac{\sum_{j=1}^{r} F\left(\sigma_{j}\left(g_{m_{i}}-g\right)\right)}{r} \\
& =\frac{1}{r \mu_{k}(D)}\left[\int_{\left\{\left\|g_{m_{i}}-g\right\|<\varepsilon\right\}} \sum_{j=1}^{r} F\left(\sigma_{j}\left(g_{m_{i}}-g\right)\right)+\int_{\left\{\left\|g_{m_{i}}-g\right\| \geq \varepsilon\right\}} \sum_{j=1}^{r} F\left(\sigma_{j}\left(g_{m_{i}}-g\right)\right)\right] \\
& \leq \frac{1}{r \mu_{k}(D)}\left[\int_{\left\{\left\|g_{m_{i}}-g\right\|<\varepsilon\right\}} r+\int_{\left\{\left\|g_{m_{i}}-g\right\| \geq \varepsilon\right\}}(r-1)\right] \\
& \leq \frac{r \mu_{k}\left\{\left\|g_{m_{i}}-g\right\|<\varepsilon\right\}+(r-1) \mu_{k}\left\{\left\|g_{m_{i}}-g\right\| \geq \varepsilon\right\}}{r \mu_{k}(D)} \\
& =1-\frac{\mu_{k}\left\{\left\|g_{m_{i}}-g\right\| \geq \varepsilon\right\}}{r \mu_{k}(D)} \leq F(0)-\frac{\delta}{r \mu_{k}(D)}
\end{aligned}
$$

which is a contradiction to (2.5).

REMARK 2.6. Let $f: D \rightarrow \mathbb{C}^{r \times r}$ be a measurable function defined on a set $D \subset \mathbb{R}^{k}$ with $0<\mu_{k}(D)<\infty$, and assume that

$$
\frac{1}{\mu_{k}(D)} \int_{D} \frac{\sum_{j=1}^{r} F\left(\sigma_{j}(f)\right)}{r}=F(0), \quad \forall F \in C_{c}(\mathbb{R}) .
$$

Then $f=O_{r}$ a.e. Indeed, by Lemma 2.5, the previous equation implies that $f \rightarrow O_{r}$ in measure, i.e., $f=O_{r}$ a.e.

2.3.5. Riemann-integrable functions. A function $a:[0,1] \rightarrow \mathbb{C}$ is said to be Riemannintegrable if its real and imaginary parts $\Re(a), \Im(a):[0,1] \rightarrow \mathbb{R}$ are Riemann-integrable in the classical sense. Recall that any Riemann-integrable function is bounded by definition. A matrix-valued function $a:[0,1] \rightarrow \mathbb{C}^{r \times r}$ is said to be Riemann-integrable if its components $a_{\alpha \beta}:[0,1] \rightarrow \mathbb{C}, \alpha, \beta=1, \ldots, r$, are Riemann-integrable. We report below a list of properties possessed by Riemann-integrable functions that will be used in this work, either explicitly or implicitly.

- If $\alpha, \beta \in \mathbb{C}$ and $a, b:[0,1] \rightarrow \mathbb{C}$ are Riemann-integrable, then $\alpha a+\beta b$ is Riemannintegrable.

- If $a, b:[0,1] \rightarrow \mathbb{C}$ are Riemann-integrable, then $a b$ is Riemann-integrable.

- If $a:[0,1] \rightarrow \mathbb{C}$ is Riemann-integrable and $F: \mathbb{C} \rightarrow \mathbb{C}$ is continuous, then $F(a):[0,1] \rightarrow \mathbb{C}$ is Riemann-integrable. 
- If $a:[0,1] \rightarrow \mathbb{C}$ is Riemann-integrable, then $a$ belongs to $L^{\infty}([0,1])$ and its Lebesgue and Riemann integrals over $[0,1]$ coincide.

- If $a:[0,1] \rightarrow \mathbb{C}$ is bounded, then $a$ is Riemann-integrable if and only if $a$ is continuous a.e. Note that the last two properties imply the first three. The proof of the second-to-last property can be found in [69, pp. 73-74] or [16, Theorem 2.10.1]. The last property is Lebesgue's characterization theorem of Riemann-integrable functions [69, p. 104]. A further property of Riemann-integrable functions that will be used in this work is reported in the next lemma [50, Lemma 2.9].

Lemma 2.7. Let $a:[0,1] \rightarrow \mathbb{R}$ be Riemann-integrable. For each $n \in \mathbb{N}$, consider the partition of $(0,1]$ given by the intervals

$$
I_{i, n}=\left(\frac{i-1}{n}, \frac{i}{n}\right], \quad i=1, \ldots, n,
$$

and let

$$
a_{i, n} \in\left[\inf _{x \in I_{i, n}} a(x), \sup _{x \in I_{i, n}} a(x)\right], \quad i=1, \ldots, n
$$

Then

$$
\sum_{i=1}^{n} a_{i, n} \chi_{I_{i, n}} \rightarrow a \quad \text { a.e. in }[0,1], \quad \lim _{n \rightarrow \infty} \frac{1}{n} \sum_{i=1}^{n} a_{i, n}=\int_{0}^{1} a(x) \mathrm{d} x .
$$

2.4. Singular value and eigenvalue distribution of a sequence of matrices.

2.4.1. The notion of singular value and eigenvalue distribution. We here introduce the fundamental definitions of singular value and eigenvalue (or spectral) distribution for a given sequence of matrices.

DEFINITION 2.8 (Singular value and eigenvalue distribution of a sequence of matrices). Let $\left\{A_{n}\right\}_{n}$ be a sequence of matrices with $A_{n}$ of size $d_{n}$, and let $f: D \subset \mathbb{R}^{k} \rightarrow \mathbb{C}^{r \times r}$ be a measurable matrix-valued function defined on a set $D$ with $0<\mu_{k}(D)<\infty$.

- We say that $\left\{A_{n}\right\}_{n}$ has a (asymptotic) singular value distribution described by $f$, and we write $\left\{A_{n}\right\}_{n} \sim_{\sigma} f$, if

$$
\lim _{n \rightarrow \infty} \frac{1}{d_{n}} \sum_{j=1}^{d_{n}} F\left(\sigma_{j}\left(A_{n}\right)\right)=\frac{1}{\mu_{k}(D)} \int_{D} \frac{\sum_{i=1}^{r} F\left(\sigma_{i}(f(\mathbf{x}))\right)}{r} \mathrm{~d} \mathbf{x}, \quad \forall F \in C_{c}(\mathbb{R}) .
$$

In this case, the function $f$ is referred to as the singular value symbol of $\left\{A_{n}\right\}_{n}$.

- We say that $\left\{A_{n}\right\}_{n}$ has an (asymptotic) eigenvalue (or spectral) distribution described by $f$, and we write $\left\{A_{n}\right\}_{n} \sim_{\lambda} f$, if

$$
\lim _{n \rightarrow \infty} \frac{1}{d_{n}} \sum_{j=1}^{d_{n}} F\left(\lambda_{j}\left(A_{n}\right)\right)=\frac{1}{\mu_{k}(D)} \int_{D} \frac{\sum_{i=1}^{r} F\left(\lambda_{i}(f(\mathbf{x}))\right)}{r} \mathrm{~d} \mathbf{x}, \quad \forall F \in C_{c}(\mathbb{C}) .
$$

In this case, the function $f$ is referred to as the eigenvalue (or spectral) symbol of $\left\{A_{n}\right\}_{n}$.

Note that Definition 2.8 is well-posed by Lemma 2.1, which ensures that the functions $\mathbf{x} \mapsto \sum_{i=1}^{r} F\left(\sigma_{i}(f(\mathbf{x}))\right)$ and $\mathbf{x} \mapsto \sum_{i=1}^{r} F\left(\lambda_{i}(f(\mathbf{x}))\right)$ are measurable. In this work, whenever we write a relation such as $\left\{A_{n}\right\}_{n} \sim_{\sigma} f$ or $\left\{A_{n}\right\}_{n} \sim_{\lambda} f$, it is understood that $f$ is as in Definition 2.8, that is, $f$ is a measurable function taking values in $\mathbb{C}^{r \times r}$ for some $r \geq 1$ and defined on a subset $D$ of some $\mathbb{R}^{k}$ with $0<\mu_{k}(D)<\infty$.

REMARK 2.9 (Informal meaning of the singular value and eigenvalue distribution). The informal meaning behind the spectral distribution (2.7) is the following: assuming that $f$ 
possesses $r$ a.e. continuous eigenvalue functions $\lambda_{i}(f(\mathbf{x})), i=1, \ldots, r$, then the eigenvalues of $A_{n}$, except possibly for $o\left(d_{n}\right)$ outliers, can be subdivided into $r$ different subsets of approximately the same cardinality, and, for $n$ large enough, the eigenvalues belonging to the $i$ th subset are approximately equal to the samples of the $i$ th eigenvalue function $\lambda_{i}(f(\mathbf{x}))$ over a uniform grid in the domain $D$. For instance, if $k=1, d_{n}=n r$, and $D=[a, b]$, then, assuming we have no outliers, the eigenvalues of $A_{n}$ are approximately equal to

$$
\lambda_{i}\left(f\left(a+j \frac{b-a}{n}\right)\right), \quad j=1, \ldots, n, \quad i=1, \ldots, r,
$$

for $n$ large enough. Similarly, if $k=2, d_{n}=n^{2} r$, and $D=\left[a_{1}, b_{1}\right] \times\left[a_{2}, b_{2}\right]$, then, assuming we have no outliers, the eigenvalues of $A_{n}$ are approximately equal to

$$
\lambda_{i}\left(f\left(a_{1}+j_{1} \frac{b_{1}-a_{1}}{n}, a_{2}+j_{2} \frac{b_{2}-a_{2}}{n}\right)\right), \quad j_{1}, j_{2}=1, \ldots, n, \quad i=1, \ldots, r,
$$

for $n$ large enough; and so on for $k \geq 3$. A completely analogous meaning can also be given for the singular value distribution (2.6).

REMARK 2.10 (Rearrangement). Let $D=\left[a_{1}, b_{1}\right] \times \cdots \times\left[a_{k}, b_{k}\right] \subset \mathbb{R}^{k}$, and let $f: D \rightarrow \mathbb{C}^{r \times r}$ be a measurable function possessing $r$ real-valued a.e. continuous eigenvalue functions $\lambda_{i}(f(\mathbf{x})), i=1, \ldots, r$. Compute for each $\rho \in \mathbb{N}$ the uniform samples

$$
\lambda_{i}\left(f\left(a_{1}+j_{1} \frac{b_{1}-a_{1}}{\rho}, \ldots, a_{k}+j_{k} \frac{b_{k}-a_{k}}{\rho}\right)\right), \quad j_{1}, \ldots, j_{k}=1, \ldots, \rho, \quad i=1, \ldots, r,
$$

sort them in non-decreasing order, and put them into a vector $\left(\varsigma_{1}, \varsigma_{2}, \ldots, \varsigma_{r} \rho^{k}\right)$. Let $\phi_{\rho}:[0,1] \rightarrow \mathbb{R}$ be the piecewise linear non-decreasing function that interpolates the samples $\left(\varsigma_{0}=\varsigma_{1}, \varsigma_{1}, \varsigma_{2}, \ldots, \varsigma_{r \rho^{k}}\right)$ over the nodes $\left(0, \frac{1}{r \rho^{k}}, \frac{2}{r \rho^{k}}, \ldots, 1\right)$, i.e.,

$$
\begin{cases}\phi_{\rho}\left(\frac{i}{r \rho^{k}}\right)=\varsigma_{i}, & i=0, \ldots, r \rho^{k}, \\ \phi_{\rho} \text { linear on }\left[\frac{i}{r \rho^{k}}, \frac{i+1}{r \rho^{k}}\right], & i=0, \ldots, r \rho^{k}-1 .\end{cases}
$$

When $\rho \rightarrow \infty$, the function $\phi_{\rho}$ converges a.e. to a function $\phi$, which is non-decreasing on $(0,1)$ and satisfies

$$
\int_{0}^{1} F(\phi(t)) \mathrm{d} t=\frac{1}{\mu_{k}(D)} \int_{D} \frac{\sum_{i=1}^{r} F\left(\lambda_{i}(f(\mathbf{x}))\right)}{r} \mathrm{~d} \mathbf{x}, \quad \forall F \in C_{c}(\mathbb{C}) .
$$

The proof of this result is omitted because it is rather technical; it involves arguments from [50, solution of Exercise 3.1] and [6]. The function $\phi$ is referred to as the canonical rearranged version of $f$. What is interesting about $\phi$ is that, by (2.8), if $\left\{A_{n}\right\}_{n} \sim_{\lambda} f$, then $\left\{A_{n}\right\}_{n} \sim_{\lambda} \phi$, i.e., if $f$ is a spectral symbol of $\left\{A_{n}\right\}_{n}$, then the same is true for $\phi$. Moreover, $\phi$ is a univariate non-decreasing scalar function, and hence it is much easier to handle than $f$. According to Remark 2.9, if we have $\left\{A_{n}\right\}_{n} \sim_{\lambda} f$ (and hence also $\left\{A_{n}\right\}_{n} \sim_{\lambda} \phi$ ), then, for $n$ large enough, the eigenvalues of $A_{n}$, with the possible exception of $o\left(d_{n}\right)$ outliers, are approximately equal to the samples of $\phi$ over a uniform grid in $[0,1]$. Precise error estimates as well as an analysis under suitable assumptions may be produced by following [19] and the references therein, possibly also considering the numerics in [44].

REMARK 2.11 (Canonical rearranged version and quantile function). The canonical rearranged version $\phi$, under the different name of quantile function, was carefully considered in $[17,18]$. In a less systematic way, the same notion was introduced in [33], when 
dealing with the spectral analysis of preconditioning strategies for large Toeplitz linear systems. According to the generalization presented in [18], consider the probability space $(\Omega, \mathcal{F}, \mathrm{P})$, where $\Omega=D \times\{1,2, \ldots, r\}$ and $\mathrm{P}$ is the uniform probability over $\Omega$ defined as the product of the normalized Lebesgue measure on $D$ and the normalized counting measure on $\{1,2, \ldots, r\}$. If we define the $\mathcal{F}$-measurable function (random variable) $X: \Omega \rightarrow \mathbb{R}$ by setting $X(\mathbf{x}, i)=\lambda_{i}(f(\mathbf{x}))$, then $\phi$ coincides a.e. with the quantile function associated with $X$, i.e., $Q_{X}(p)=\inf \{v \in \mathbb{R}: \mathrm{P}(X<v) \geq p\}$.

2.4.2. Clustering and attraction. In what follows, if $S \subseteq \mathbb{C}$ and $\varepsilon>0$, we denote by $D(S, \varepsilon)$ the $\varepsilon$-expansion of $S$, which is defined as $D(S, \varepsilon)=\bigcup_{z \in S} D(z, \varepsilon)$.

DEFINITION 2.12 (Clustering of a sequence of matrices). Let $\left\{A_{n}\right\}_{n}$ be a sequence of matrices with $A_{n}$ of size $d_{n}$, and let $S \subseteq \mathbb{C}$ be a nonempty subset of $\mathbb{C}$.

- We say that $\left\{A_{n}\right\}_{n}$ is strongly clustered at $S$ (in the sense of the eigenvalues), or equivalently, that the eigenvalues of $\left\{A_{n}\right\}_{n}$ are strongly clustered at $S$, if, for every $\varepsilon>0$, the number of eigenvalues of $A_{n}$ lying outside $D(S, \varepsilon)$ is bounded by a constant $C_{\varepsilon}$ independent of $n$; that is, for every $\varepsilon>0$,

$$
\#\left\{j \in\left\{1, \ldots, d_{n}\right\}: \lambda_{j}\left(A_{n}\right) \notin D(S, \varepsilon)\right\}=O(1) .
$$

- We say that $\left\{A_{n}\right\}_{n}$ is weakly clustered at $S$ (in the sense of the eigenvalues), or equivalently, that the eigenvalues of $\left\{A_{n}\right\}_{n}$ are weakly clustered at $S$, if, for every $\varepsilon>0$,

$$
\#\left\{j \in\left\{1, \ldots, d_{n}\right\}: \lambda_{j}\left(A_{n}\right) \notin D(S, \varepsilon)\right\}=o\left(d_{n}\right) .
$$

By replacing "eigenvalues" with "singular values" and $\lambda_{j}\left(A_{n}\right)$ with $\sigma_{j}\left(A_{n}\right)$ in (2.9)-(2.10), we obtain the definitions of a sequence of matrices strongly or weakly clustered at a nonempty subset of $\mathbb{C}$ in the sense of the singular values.

Throughout this work, when we speak of strong/weak cluster, a sequence of matrices strongly/weakly clustered, etc., without further specifications, it is understood "in the sense of the eigenvalues". When the clustering is intended in the sense of the singular values, this is specified every time.

DEFINITION 2.13 (Spectral attraction). Let $\left\{A_{n}\right\}_{n}$ be a sequence of matrices with $A_{n}$ of size $d_{n}$, and let $z \in \mathbb{C}$. We say that $z$ strongly attracts the spectrum $\Lambda\left(A_{n}\right)$ with infinite order if, once we have ordered the eigenvalues of $A_{n}$ according to their distance from $z$,

$$
\left|\lambda_{1}\left(A_{n}\right)-z\right| \leq\left|\lambda_{2}\left(A_{n}\right)-z\right| \leq \ldots \leq\left|\lambda_{d_{n}}\left(A_{n}\right)-z\right|,
$$

the following limit relation holds for each fixed $j \geq 1$ :

$$
\lim _{n \rightarrow \infty}\left|\lambda_{j}\left(A_{n}\right)-z\right|=0 .
$$

THEOREM 2.14. If $\left\{A_{n}\right\}_{n} \sim_{\lambda} f$, then $\left\{A_{n}\right\}_{n}$ is weakly clustered at the essential range $\mathcal{E} \mathcal{R}(f)$ and every point of $\mathcal{E} \mathcal{R}(f)$ strongly attracts the spectrum $\Lambda\left(A_{n}\right)$ with infinite order.

Proof. Denote by $D \subset \mathbb{R}^{k}$ and $\mathbb{C}^{r \times r}$ the domain and codomain of $f$, respectively, and let $d_{n}$ be the size of $A_{n}$. Set $S=\mathcal{E} \mathcal{R}(f)$ and fix $\varepsilon>0$. For any $\delta>0$, let $F_{\varepsilon, \delta}$ be a function in $C_{c}(\mathbb{C})$ such that

$$
\begin{cases}0 \leq F_{\varepsilon, \delta} \leq 1 & \text { over } \mathbb{C}, \\ F_{\varepsilon, \delta}=1 & \text { over } S \cap \overline{D(0,1 / \delta)}, \\ F_{\varepsilon, \delta}=0 & \text { outside } D(S, \varepsilon) .\end{cases}
$$


Note that such a function exists by Urysohn's lemma [71, Lemma 2.12] because $S \cap \overline{D(0,1 / \delta)}$ is a compact set contained in the open set $D(S, \varepsilon)$ (recall that $S$ is closed by Lemma 2.3). Clearly, we have $F_{\varepsilon, \delta} \leq \chi_{D(S, \varepsilon)}$, hence

$$
\begin{aligned}
& \frac{\#\left\{j \in\left\{1, \ldots, d_{n}\right\}: \lambda_{j}\left(A_{n}\right) \notin D(S, \varepsilon)\right\}}{d_{n}} \\
& =1-\frac{\#\left\{j \in\left\{1, \ldots, d_{n}\right\}: \lambda_{j}\left(A_{n}\right) \in D(S, \varepsilon)\right\}}{d_{n}} \\
& =1-\frac{1}{d_{n}} \sum_{j=1}^{d_{n}} \chi_{D(S, \varepsilon)}\left(\lambda_{j}\left(A_{n}\right)\right) \leq 1-\frac{1}{d_{n}} \sum_{j=1}^{d_{n}} F_{\varepsilon, \delta}\left(\lambda_{j}\left(A_{n}\right)\right) .
\end{aligned}
$$

Passing to the limit as $n \rightarrow \infty$ and using the assumption $\left\{A_{n}\right\}_{n} \sim_{\lambda} f$, we obtain

$$
\begin{gathered}
\limsup _{n \rightarrow \infty} \frac{\#\left\{j \in\left\{1, \ldots, d_{n}\right\}: \lambda_{j}\left(A_{n}\right) \notin D(S, \varepsilon)\right\}}{d_{n}} \\
\quad \leq 1-\frac{1}{\mu_{k}(D)} \int_{D} \frac{\sum_{j=1}^{r} F_{\varepsilon, \delta}\left(\lambda_{j}(f(\mathbf{x}))\right)}{r} \mathrm{~d} \mathbf{x},
\end{gathered}
$$

for every $\delta>0$. To complete the proof that $\left\{A_{n}\right\}_{n}$ is weakly clustered at $S$, we show that

$$
\lim _{\delta \rightarrow 0} \int_{D} \frac{\sum_{j=1}^{r} F_{\varepsilon, \delta}\left(\lambda_{j}(f(\mathbf{x}))\right)}{r} \mathrm{~d} \mathbf{x}=\mu_{k}(D) .
$$

Since $F_{\varepsilon, \delta} \rightarrow 1$ pointwise over $S$ as $\delta \rightarrow 0$ and $\Lambda(f) \subseteq S$ a.e. by Lemma 2.3,

$$
\frac{\sum_{j=1}^{r} F_{\varepsilon, \delta}\left(\lambda_{j}(f(\mathbf{x}))\right)}{r} \rightarrow 1 \quad \text { a.e. in } D .
$$

Hence, (2.11) follows immediately from the dominated convergence theorem.

To show that each point of $S$ strongly attracts $\Lambda\left(A_{n}\right)$ with infinite order, fix a point $s \in S$. For any $\varepsilon>0$, take $F_{\varepsilon} \in C_{c}(\mathbb{C})$ such that $0 \leq F_{\varepsilon} \leq 1$ over $\mathbb{C}, F_{\varepsilon}=1$ over $D(s, \varepsilon)$ and $F_{\varepsilon}=0$ outside $D(s, 2 \varepsilon)$. Since $\chi_{D(s, \varepsilon)} \leq F_{\varepsilon} \leq \chi_{D(s, 2 \varepsilon)}$ and $\left\{A_{n}\right\}_{n} \sim_{\lambda} f$, we have

$$
\begin{aligned}
& \frac{\#\left\{j \in\left\{1, \ldots, d_{n}\right\}: \lambda_{j}\left(A_{n}\right) \in D(s, 2 \varepsilon)\right\}}{d_{n}} \geq \frac{1}{d_{n}} \sum_{j=1}^{d_{n}} F_{\varepsilon}\left(\lambda_{j}\left(A_{n}\right)\right) \\
& \stackrel{n \rightarrow \infty}{\longrightarrow} \frac{1}{\mu_{k}(D)} \int_{D} \frac{\sum_{j=1}^{r} F_{\varepsilon}\left(\lambda_{j}(f(\mathbf{x}))\right)}{r} \mathrm{~d} \mathbf{x} \geq \frac{\mu_{k}\left\{\exists j \in\{1, \ldots, r\}: \lambda_{j}(f) \in D(s, \varepsilon)\right\}}{r \mu_{k}(D)} .
\end{aligned}
$$

Passing to the limit as $n \rightarrow \infty$, we obtain

$$
\begin{gathered}
\liminf _{n \rightarrow \infty} \frac{\#\left\{j \in\left\{1, \ldots, d_{n}\right\}: \lambda_{j}\left(A_{n}\right) \in D(s, 2 \varepsilon)\right\}}{d_{n}} \\
\geq \frac{\mu_{k}\left\{\exists j \in\{1, \ldots, r\}: \lambda_{j}(f) \in D(s, \varepsilon)\right\}}{r \mu_{k}(D)} .
\end{gathered}
$$

By definition of the essential range, the right-hand side of (2.12) is positive for every $\varepsilon>0$. This implies that $s$ strongly attracts $\Lambda\left(A_{n}\right)$ with infinite order.

COROLlary 2.15. If $\left\{A_{n}\right\}_{n} \sim_{\lambda} f$ and $\Lambda\left(A_{n}\right)$ is contained in $S \subseteq \mathbb{C}$ for all $n$, then $\mathcal{E} \mathcal{R}(f)$ is contained in the closure $\bar{S}$.

Proof. By Theorem 2.14, each point of $\mathcal{E R}(f)$ strongly attracts $\Lambda\left(A_{n}\right)$ with infinite order. If $z \notin \bar{S}$, there exists a disk $D(z, \varepsilon)$ which does not intersect $\bar{S}$. Since $\Lambda\left(A_{n}\right) \subseteq S$ for all $n$, it is clear that $z$ cannot attract $\Lambda\left(A_{n}\right)$ with infinite order, hence $z \notin \mathcal{E R}(f)$. We conclude that $\mathcal{E R}(f) \subseteq \bar{S}$ 
2.4.3. Zero-distributed sequences. A sequence of matrices $\left\{Z_{n}\right\}_{n}$ with $Z_{n}$ of size $d_{n}$ is said to be zero-distributed if $\left\{Z_{n}\right\}_{n} \sim_{\sigma} 0$, i.e.,

$$
\lim _{n \rightarrow \infty} \frac{1}{d_{n}} \sum_{j=1}^{d_{n}} F\left(\sigma_{j}\left(Z_{n}\right)\right)=F(0), \quad \forall F \in C_{c}(\mathbb{R}) .
$$

It is clear that, for any $r \geq 1,\left\{Z_{n}\right\}_{n} \sim_{\sigma} 0$ is equivalent to $\left\{Z_{n}\right\}_{n} \sim_{\sigma} O_{r}$. Theorem 2.16 provides a characterization of zero-distributed sequences together with a sufficient condition for detecting such sequences. For the related proof, see [50, Theorems 3.2 and 3.3].

THEOREM 2.16. Let $\left\{Z_{n}\right\}_{n}$ be a sequence of matrices with $Z_{n}$ of size $d_{n}$.

1. $\left\{Z_{n}\right\}_{n} \sim_{\sigma} 0$ if and only if $Z_{n}=R_{n}+N_{n}$ with $\lim _{n \rightarrow \infty}\left(\operatorname{rank}\left(R_{n}\right) / d_{n}\right)=\lim _{n \rightarrow \infty}\left\|N_{n}\right\|=0$. 2. $\left\{Z_{n}\right\}_{n} \sim_{\sigma} 0$ if there exists $p \in[1, \infty)$ such that $\lim _{n \rightarrow \infty}\left(\left\|Z_{n}\right\|_{p}^{p} / d_{n}\right)=0$.

2.4.4. Sparsely unbounded and sparsely vanishing sequences of matrices. The notions of sparsely unbounded and sparsely vanishing sequences of matrices play an important role within the framework of the theory of block GLT sequences.

DEFINITION 2.17 (Sparsely unbounded sequence of matrices). A sequence of matrices $\left\{A_{n}\right\}_{n}$ with $A_{n}$ of size $d_{n}$ is said to be sparsely unbounded (s.u.) if for every $M>0$ there exists $n_{M}$ such that, for $n \geq n_{M}$,

$$
\frac{\#\left\{i \in\left\{1, \ldots, d_{n}\right\}: \sigma_{i}\left(A_{n}\right)>M\right\}}{d_{n}} \leq r(M),
$$

where $\lim _{M \rightarrow \infty} r(M)=0$.

For the proofs of the next three propositions, we refer the reader to Proposition 5.3 of [50] and Propositions 2.2 and 2.3 of [55]. Note that the proof in [50] is made for $d_{n}=n$ and the proofs in [55] are made for $d_{n}=s n$ for a fixed $s \in \mathbb{N}$, but the extension to the case of a general $d_{n}$ tending to infinity is straightforward.

PROPOSITION 2.18. Let $\left\{A_{n}\right\}_{n}$ be a sequence of matrices with $A_{n}$ of size $d_{n}$. The following are equivalent.

1. $\left\{A_{n}\right\}_{n}$ is s.u.

2. $\lim _{M \rightarrow \infty} \limsup _{n \rightarrow \infty} \frac{\#\left\{i \in\left\{1, \ldots, d_{n}\right\}: \sigma_{i}\left(A_{n}\right)>M\right\}}{d_{n}}=0$.

3. For every $M>0$ there exists $n_{M}$ such that, for $n \geq n_{M}$,

$$
\begin{aligned}
& \quad A_{n}=\hat{A}_{n, M}+\tilde{A}_{n, M}, \quad \operatorname{rank}\left(\hat{A}_{n, M}\right) \leq r(M) d_{n}, \quad\left\|\tilde{A}_{n, M}\right\| \leq M, \\
& \text { where } \lim _{M \rightarrow \infty} r(M)=0 .
\end{aligned}
$$

PROPOSITION 2.19. If $\left\{A_{n}\right\}_{n},\left\{A_{n}^{\prime}\right\}_{n}$ are s.u., then $\left\{A_{n} A_{n}^{\prime}\right\}_{n}$ is s.u.

PROPOSITION 2.20. If $\left\{A_{n}\right\}_{n} \sim_{\sigma} f$, then $\left\{A_{n}\right\}_{n}$ is s.u.

REMARK 2.21. Let $\left\{A_{n}\right\}_{n}$ be an s.u. sequence of Hermitian matrices with $A_{n}$ of size $d_{n}$. Then, the following stronger version of condition 3 in Proposition 2.18 is satisfied: for every $M>0$ there exists $n_{M}$ such that, for $n \geq n_{M}$,

$$
A_{n}=\hat{A}_{n, M}+\tilde{A}_{n, M}, \quad \operatorname{rank}\left(\hat{A}_{n, M}\right) \leq r(M) d_{n}, \quad\left\|\tilde{A}_{n, M}\right\| \leq M,
$$

where $\lim _{M \rightarrow \infty} r(M)=0$, the matrices $\hat{A}_{n, M}$ and $\tilde{A}_{n, M}$ are Hermitian, and for all functions $g: \mathbb{R} \rightarrow \mathbb{R}$ satisfying $g(0)=0$ we have

$$
g\left(\hat{A}_{n, M}+\tilde{A}_{n, M}\right)=g\left(\hat{A}_{n, M}\right)+g\left(\tilde{A}_{n, M}\right) .
$$




\section{ETNA}

Kent State University and

Johann Radon Institute (RICAM)

This stronger version of condition 3 has been proved in [50, p. 157 (lines 21-34) and p. 158 (lines 1-8)] for the case " $d_{n}=n$ ", but the extension to the general case " $d_{n} \rightarrow \infty$ as $n \rightarrow \infty$ " is immediate. ${ }^{3}$

Strictly related to the notion of sparsely unbounded sequences of matrices is the notion of sparsely vanishing sequences of matrices.

DEFINITION 2.22 (Sparsely vanishing sequence of matrices). A sequence of matrices $\left\{A_{n}\right\}_{n}$ with $A_{n}$ of size $d_{n}$ is said to be sparsely vanishing (s.v.) if for every $M>0$ there exists $n_{M}$ such that, for $n \geq n_{M}$,

$$
\frac{\#\left\{i \in\left\{1, \ldots, d_{n}\right\}: \sigma_{i}\left(A_{n}\right)<1 / M\right\}}{d_{n}} \leq r(M),
$$

where $\lim _{M \rightarrow \infty} r(M)=0$.

REMARK 2.23. If $\left\{A_{n}\right\}_{n}$ is s.v., then the sequence of Moore-Penrose pseudoinverses $\left\{A_{n}^{\dagger}\right\}_{n}$ is s.u. This follows immediately from the fact that the singular values of $A^{\dagger}$ are given by $1 / \sigma_{1}(A), \ldots, 1 / \sigma_{r}(A), 0, \ldots, 0$, where $\sigma_{1}(A), \ldots, \sigma_{r}(A)$ are the nonzero singular values of $A(r=\operatorname{rank}(A))$.

REMARK 2.24. A sequence of matrices $\left\{A_{n}\right\}_{n}$ with $A_{n}$ of size $d_{n}$ is s.v. if and only if

$$
\lim _{M \rightarrow \infty} \limsup _{n \rightarrow \infty} \frac{\#\left\{i \in\left\{1, \ldots, d_{n}\right\}: \sigma_{i}\left(A_{n}\right)<1 / M\right\}}{d_{n}}=0 ;
$$

see [50, Remark 8.6].

Proposition 2.25 is the analog to Proposition 2.20 for s.v. sequences of matrices [56, Proposition 2.3].

PROPOSITION 2.25. If $\left\{A_{n}\right\}_{n} \sim_{\sigma} f$, then $\left\{A_{n}\right\}_{n}$ is s.v. if and only if $f$ is invertible a.e.

2.4.5. Spectral distribution of sequences of perturbed/compressed/expanded Hermitian matrices. Theorem 2.26 reports from [8] a recent important result about the spectral distribution of sequences of perturbed Hermitian matrices. It nicely extends previous results obtained in $[53,58]$.

THEOREM 2.26. Let $\left\{X_{n}\right\}_{n},\left\{Y_{n}\right\}_{n}$ be sequences of matrices with $X_{n}, Y_{n}$ of size $d_{n}$, and set $A_{n}=X_{n}+Y_{n}$. Assume that the following conditions are met.

1. Every $X_{n}$ is Hermitian and $\left\{X_{n}\right\}_{n} \sim_{\lambda} f$.

2. $\left\|Y_{n}\right\|_{2}=o\left(\sqrt{d_{n}}\right)$.

Then $\left\{A_{n}\right\}_{n} \sim_{\lambda} f$.

REMARK 2.27. If $\left\|Y_{n}\right\| \leq C$ for some constant $C$ independent of $n$ and $\left\|Y_{n}\right\|_{1}=o\left(d_{n}\right)$, then $Y_{n}$ satisfies the second assumption in Theorem 2.26 by (2.4).

Theorem 2.28 concerns the singular value and spectral distribution of sequences of matrices obtained as a compression (or expansion) of another sequence of matrices. For the proof, we refer the reader to [62, Theorem 4.3 and Corollary 4.4].

THEOREM 2.28. Let $\left\{X_{n}\right\}_{n}$ be a sequence of matrices with $X_{n}$ of size $d_{n}$, and let $\left\{P_{n}\right\}_{n}$ be a sequence such that $P_{n} \in \mathbb{C}^{d_{n} \times \delta_{n}}, P_{n}^{*} P_{n}=I_{\delta_{n}}, \delta_{n} \leq d_{n}$, and $\delta_{n} / d_{n} \rightarrow 1$ as $n \rightarrow \infty$.

1. We have $\left\{X_{n}\right\}_{n} \sim_{\sigma} f$ if and only if $\left\{P_{n}^{*} X_{n} P_{n}\right\}_{n} \sim_{\sigma} f$.

2. In the case where the matrices $X_{n}$ are Hermitian, we have $\left\{X_{n}\right\}_{n} \sim_{\lambda} f$ if and only if $\left\{P_{n}^{*} X_{n} P_{n}\right\}_{n} \sim_{\lambda} f$.

\footnotetext{
${ }^{3}$ Note that the condition $g(0)=0$ is missing in [50], but it is actually necessary. Luckily, the absence of this condition does not affect the validity of [50, proof of Theorem 8.9] because, by replacing therein $p_{m, M}$ with $p_{m, M}+f(0)-p_{m, M}(0)$ if necessary, it may be assumed without loss of generality that $p_{m, M}(0)=f(0)$, so that the function $g=f-p_{m, M}$ satisfies $g(0)=0$.
} 


\subsection{Approximating classes of sequences.}

2.5.1. Definition of a.c.s. and the a.c.s. topology $\tau_{\text {a.c.s. }}$ The formal definition of a.c.s. is given below.

DEFINITION 2.29 (Approximating class of sequences). Let $\left\{A_{n}\right\}_{n}$ be a sequence of matrices with $A_{n}$ of size $d_{n}$, and let $\left\{\left\{B_{n, m}\right\}_{n}\right\}_{m}$ be a sequence of sequences of matrices with $B_{n, m}$ of size $d_{n}$. We say that $\left\{\left\{B_{n, m}\right\}_{n}\right\}_{m}$ is an approximating class of sequences (a.c.s.) for $\left\{A_{n}\right\}_{n}$ if the following condition is met: for every $m$ there exists $n_{m}$ such that, for $n \geq n_{m}$,

$$
A_{n}=B_{n, m}+R_{n, m}+N_{n, m}, \quad \operatorname{rank}\left(R_{n, m}\right) \leq c(m) d_{n}, \quad\left\|N_{n, m}\right\| \leq \omega(m),
$$

where $n_{m}, c(m), \omega(m)$ depend only on $m$ and $\lim _{m \rightarrow \infty} c(m)=\lim _{m \rightarrow \infty} \omega(m)=0$.

Roughly speaking, $\left\{\left\{B_{n, m}\right\}_{n}\right\}_{m}$ is an a.c.s. for $\left\{A_{n}\right\}_{n}$ if, for all sufficiently large $m$, the sequence $\left\{B_{n, m}\right\}_{n}$ approximates (asymptotically) the sequence $\left\{A_{n}\right\}_{n}$ in the sense that $A_{n}$ is eventually equal to $B_{n, m}$ plus a small-rank matrix (with respect to the matrix size $d_{n}$ ) plus a small-norm matrix.

It turns out that, for each fixed sequence of positive integers $d_{n}$ such that $d_{n} \rightarrow \infty$, the notion of a.c.s. is a notion of convergence in the space of all sequences of matrices corresponding to $\left\{d_{n}\right\}_{n}$, i.e.,

$$
\mathscr{E}=\left\{\left\{A_{n}\right\}_{n}: A_{n} \in \mathbb{C}^{d_{n} \times d_{n}} \text { for every } n\right\} .
$$

To be precise, for every $\varphi:[0, \infty) \rightarrow[0, \infty)$ and every square matrix $A \in \mathbb{C}^{\ell \times \ell}$, let

$$
p^{\varphi}(A)=\frac{1}{\ell} \sum_{i=1}^{\ell} \varphi\left(\sigma_{i}(A)\right)
$$

and define

$$
\begin{array}{rrr}
p_{\text {a.c.s. }}^{\varphi}\left(\left\{A_{n}\right\}_{n}\right)=\limsup _{n \rightarrow \infty} p^{\varphi}\left(A_{n}\right), & \left\{A_{n}\right\}_{n} \in \mathscr{E}, \\
d_{\text {a.c.s. }}^{\varphi}\left(\left\{A_{n}\right\}_{n},\left\{B_{n}\right\}_{n}\right)=p_{\text {a.c.s. }}^{\varphi}\left(\left\{A_{n}-B_{n}\right\}_{n}\right), & \left\{A_{n}\right\}_{n},\left\{B_{n}\right\}_{n} \in \mathscr{E} .
\end{array}
$$

THEOREM 2.30. Let $\varphi:[0, \infty) \rightarrow[0, \infty)$ be a gauge function. Fix a sequence of positive integers $d_{n}$ such that $d_{n} \rightarrow \infty$, and let $\mathscr{E}$ be the space (2.13). The following properties hold. 1. $d_{\text {a.c.s. }}^{\varphi}$ in (2.14) is a complete pseudometric on $\mathscr{E}$ such that $d_{\text {a.c.s. }}^{\varphi}\left(\left\{A_{n}\right\}_{n},\left\{B_{n}\right\}_{n}\right)=0$ if and only if $\left\{A_{n}-B_{n}\right\}_{n}$ is zero-distributed.

2. Suppose $\left\{A_{n}\right\}_{n} \in \mathscr{E}$ and $\left\{\left\{B_{n, m}\right\}_{n}\right\}_{m} \subset \mathscr{E}$. Then, $\left\{\left\{B_{n, m}\right\}_{n}\right\}_{m}$ is an a.c.s. for $\left\{A_{n}\right\}_{n}$ if and only if $d_{\text {a.c.s. }}^{\varphi}\left(\left\{A_{n}\right\}_{n},\left\{B_{n, m}\right\}_{n}\right) \rightarrow 0$ as $m \rightarrow \infty$.

Theorem 2.30 was proved in [7]. It justifies the convergence notation $\left\{B_{n, m}\right\}_{n} \stackrel{\text { a.c.s. }}{\longrightarrow}$ $\left\{A_{n}\right\}_{n}$, which will be used to indicate that $\left\{\left\{B_{n, m}\right\}_{n}\right\}_{m}$ is an a.c.s. for $\left\{A_{n}\right\}_{n}$. The topology induced on $\mathscr{E}$ by the pseudometric $d_{\text {a.c.s. }}^{\varphi}$ is the same for all gauge functions $\varphi$; it is denoted by $\tau_{\text {a.c.s. }}$, and it is referred to as the a.c.s. topology. Throughout this work, we will use the notations

$$
p_{\text {a.c.s. }}=p_{\text {a.c.s. }}^{\psi}, \quad d_{\text {a.c.s. }}=d_{\text {a.c.s. }}^{\psi}, \quad \psi(x)=\frac{x}{1+x} .
$$

2.5.2. $\tau_{\text {a.c.s. }}$ and $\tau_{\text {measure }}$. Theorem 2.32 highlights important connections between $\tau_{\text {a.c.s. }}$ and $\tau_{\text {measure }}$ or, to be more precise, between the pseudometrics $d_{\text {a.c.s. }}^{\varphi}$ and $d_{\text {measure }}^{\varphi}$ inducing these two topologies. Actually, the connections between $\tau_{\text {a.c.s. }}$ and $\tau_{\text {measure }}$ are so deep that they may lead to a "bridge", in the precise mathematical sense established in [27], between measure 
theory and the asymptotic linear algebra theory underlying the notion of a.c.s.; a bridge that could be exploited to obtain matrix theory results from measure theory results and vice versa. For deeper insights on this topic, we suggest reading [7, Section 1].

LEMMA 2.31. Let $\left\{A_{n}\right\}_{n}$ be a sequence of matrices with $A_{n}$ of size $d_{n}$, and let $f: D \subset \mathbb{R}^{k} \rightarrow \mathbb{C}^{r \times r}$ be a measurable matrix-valued function defined on a set $D$ with $0<\mu_{k}(D)<\infty$. If $\left\{A_{n}\right\}_{n} \sim_{\sigma} f$, then

$$
\lim _{n \rightarrow \infty} \frac{1}{d_{n}} \sum_{j=1}^{d_{n}} F\left(\sigma_{j}\left(A_{n}\right)\right)=\frac{1}{\mu_{k}(D)} \int_{D} \frac{\sum_{i=1}^{r} F\left(\sigma_{i}(f(\mathbf{x}))\right)}{r} \mathrm{~d} \mathbf{x}
$$

for all bounded continuous functions $F: \mathbb{R} \rightarrow \mathbb{C}$.

Proof. The lemma simply says that if $\left\{A_{n}\right\}_{n} \sim_{\sigma} f$, then the limit relation (2.15) holds not only for $F \in C_{c}(\mathbb{R})$, as per Definition 2.8, but also for any continuous bounded function $F: \mathbb{R} \rightarrow \mathbb{C}$. To prove it, fix a continuous bounded function $F: \mathbb{R} \rightarrow \mathbb{C}$, and let $F_{m} \in C_{c}(\mathbb{R})$ such that $F_{m} \rightarrow F$ pointwise over $\mathbb{R}, F_{m}=F$ on $[0, m]$, and $\left\|F_{m}\right\|_{\infty} \leq\|F\|_{\infty}$. Then, for every $n, m$ we have

$$
\begin{aligned}
& \left|\frac{1}{d_{n}} \sum_{j=1}^{d_{n}} F\left(\sigma_{j}\left(A_{n}\right)\right)-\frac{1}{\mu_{k}(D)} \int_{D} \frac{\sum_{i=1}^{r} F\left(\sigma_{i}(f(\mathbf{x}))\right)}{r} \mathrm{~d} \mathbf{x}\right| \\
& \leq\left|\frac{1}{d_{n}} \sum_{j=1}^{d_{n}} F\left(\sigma_{j}\left(A_{n}\right)\right)-\frac{1}{d_{n}} \sum_{j=1}^{d_{n}} F_{m}\left(\sigma_{j}\left(A_{n}\right)\right)\right| \\
& \quad+\left|\frac{1}{d_{n}} \sum_{j=1}^{d_{n}} F_{m}\left(\sigma_{j}\left(A_{n}\right)\right)-\frac{1}{\mu_{k}(D)} \int_{D} \frac{\sum_{i=1}^{r} F_{m}\left(\sigma_{i}(f(\mathbf{x}))\right)}{r} \mathrm{~d} \mathbf{x}\right| \\
& \quad+\left|\frac{1}{\mu_{k}(D)} \int_{D} \frac{\sum_{i=1}^{r} F_{m}\left(\sigma_{i}(f(\mathbf{x}))\right)}{r} \mathrm{~d} \mathbf{x}-\frac{1}{\mu_{k}(D)} \int_{D} \frac{\sum_{i=1}^{r} F\left(\sigma_{i}(f(\mathbf{x}))\right)}{r} \mathrm{~d} \mathbf{x}\right| .
\end{aligned}
$$

The second term on the right-hand side tends to 0 as $n \rightarrow \infty$ because $\left\{A_{n}\right\}_{n} \sim_{\sigma} f$ and $F_{m} \in C_{c}(\mathbb{R})$. The third term on the right-hand side tends to 0 as $m \rightarrow \infty$ by the dominated convergence theorem since $F_{m} \rightarrow F$ pointwise and the convergence is dominated by $\left\|F_{m}-F\right\|_{\infty} \leq 2\|F\|_{\infty}$. Finally, considering that $F_{m}=F$ on $[0, m]$, the first term on the right-hand side satisfies

$$
\begin{aligned}
& \left|\frac{1}{d_{n}} \sum_{j=1}^{d_{n}} F\left(\sigma_{j}\left(A_{n}\right)\right)-\frac{1}{d_{n}} \sum_{j=1}^{d_{n}} F_{m}\left(\sigma_{j}\left(A_{n}\right)\right)\right| \\
& \quad \leq 2\|F\|_{\infty} \frac{\#\left\{j \in\left\{1, \ldots, d_{n}\right\}: \sigma_{j}\left(A_{n}\right)>m\right\}}{d_{n}} .
\end{aligned}
$$

Since $\left\{A_{n}\right\}_{n}$ is s.u. by Proposition 2.20, we have

$$
\lim _{m \rightarrow \infty} \limsup _{n \rightarrow \infty} \frac{\#\left\{j \in\left\{1, \ldots, d_{n}\right\}: \sigma_{j}\left(A_{n}\right)>m\right\}}{d_{n}}=0
$$

by Proposition 2.18. Hence, passing first to the $\lim \sup _{n \rightarrow \infty}$ and then to the $\lim _{m \rightarrow \infty}$ in (2.16), we obtain

$$
\lim _{n \rightarrow \infty}\left|\frac{1}{d_{n}} \sum_{j=1}^{d_{n}} F\left(\sigma_{j}\left(A_{n}\right)\right)-\frac{1}{\mu_{k}(D)} \int_{D} \frac{\sum_{i=1}^{r} F\left(\sigma_{i}(f(\mathbf{x}))\right)}{r} \mathrm{~d} \mathbf{x}\right|=0,
$$


as desired.

As a consequence of Lemma 2.31 and the definitions of $p_{\text {a.c.s. }}^{\varphi}$ and $p_{\text {measure, }}^{\varphi}$ we immediately obtain the following theorem.

THEOREM 2.32. If $\left\{A_{n}\right\}_{n} \sim_{\sigma} f$, then $p_{\text {a.c.s. }}^{\varphi}\left(\left\{A_{n}\right\}_{n}\right)=p_{\text {measure }}^{\varphi}(f)$ for every bounded continuous function $\varphi:[0, \infty) \rightarrow[0, \infty)$.

2.5.3. The a.c.s. tools for computing singular value and eigenvalue distributions. The importance of the a.c.s. notion resides in Theorems 2.33 and 2.34, for which we refer the reader to [55, Theorems 3.1 and 3.2].

THEOREM 2.33. Let $\left\{A_{n}\right\}_{n},\left\{B_{n, m}\right\}_{n}$ be sequences of matrices and $f, f_{m}: D \rightarrow \mathbb{C}^{r \times r}$ be measurable functions defined on a set $D \subset \mathbb{R}^{k}$ with $0<\mu_{k}(D)<\infty$. Assume that:

1. $\left\{B_{n, m}\right\}_{n} \sim_{\sigma} f_{m}$ for every $m$;

2. $\left\{B_{n, m}\right\}_{n} \stackrel{\text { a.c.s. }}{\longrightarrow}\left\{A_{n}\right\}_{n}$;

3. $f_{m} \rightarrow f$ in measure.

Then $\left\{A_{n}\right\}_{n} \sim_{\sigma} f$.

THEOREM 2.34. Let $\left\{A_{n}\right\}_{n},\left\{B_{n, m}\right\}_{n}$ be sequences of Hermitian matrices, and let $f, f_{m}: D \rightarrow \mathbb{C}^{r \times r}$ be measurable functions defined on a set $D \subset \mathbb{R}^{k}$ with $0<\mu_{k}(D)<\infty$. Assume that:

1. $\left\{B_{n, m}\right\}_{n} \sim_{\lambda} f_{m}$ for every $m$;

2. $\left\{B_{n, m}\right\}_{n} \stackrel{\text { a.c.s. }}{\longrightarrow}\left\{A_{n}\right\}_{n}$;

3. $f_{m} \rightarrow f$ in measure.

Then $\left\{A_{n}\right\}_{n} \sim_{\lambda} f$.

REMARK 2.35. Let $\left\{A_{n}\right\}_{n}$ and $\left\{B_{n}\right\}_{n}$ be sequences of matrices with $A_{n}$ and $B_{n}$ of size $d_{n}$, and suppose that $d_{\text {a.c.s. }}\left(\left\{A_{n}\right\}_{n},\left\{B_{n}\right\}_{n}\right)=0$ (which is equivalent to $\left\{A_{n}-B_{n}\right\}_{n} \sim_{\sigma} 0$ by Theorem 2.30). By Theorems 2.33 and 2.34,

- $\left\{A_{n}\right\}_{n} \sim_{\sigma} f \Longleftrightarrow\left\{B_{n}\right\}_{n} \sim_{\sigma} f$;

- if the matrices $A_{n}$ and $B_{n}$ are Hermitian, then $\left\{A_{n}\right\}_{n} \sim_{\lambda} f \Longleftrightarrow\left\{B_{n}\right\}_{n} \sim_{\lambda} f$.

2.5.4. The a.c.s. algebra. Theorem 2.36 collects important algebraic properties possessed by the a.c.s. For the proof, we refer the reader to [55, Theorem 2.3].

THEOREM 2.36. Let $\left\{A_{n}\right\}_{n},\left\{A_{n}^{\prime}\right\}_{n},\left\{B_{n, m}\right\}_{n},\left\{B_{n, m}^{\prime}\right\}_{n}$ be sequences of matrices such that $\left\{B_{n, m}\right\}_{n} \stackrel{\text { a.c.s. }}{\longrightarrow}\left\{A_{n}\right\}_{n}$ and $\left\{B_{n, m}^{\prime}\right\}_{n} \stackrel{\text { a.c.s. }}{\longrightarrow}\left\{A_{n}^{\prime}\right\}_{n}$. Then, the following properties hold.

1. $\left\{B_{n, m}^{*}\right\}_{n} \stackrel{\text { a.c.s. }}{\longrightarrow}\left\{A_{n}^{*}\right\}_{n}$.

2. $\left\{\alpha B_{n, m}+\beta B_{n, m}^{\prime}\right\}_{n} \stackrel{\text { a.c.s. }}{\longrightarrow}\left\{\alpha A_{n}+\beta A_{n}^{\prime}\right\}_{n}$ for all $\alpha, \beta \in \mathbb{C}$.

3. If $\left\{A_{n}\right\}_{n},\left\{A_{n}^{\prime}\right\}_{n}$ are s.u., then $\left\{B_{n, m} B_{n, m}^{\prime}\right\}_{n} \stackrel{\text { a.c.s. }}{\longrightarrow}\left\{A_{n} A_{n}^{\prime}\right\}_{n}$.

4. If $\left\{C_{n}\right\}_{n}$ is s.u., then $\left\{B_{n, m} C_{n}\right\}_{n} \stackrel{\text { a.c.s. }}{\longrightarrow}\left\{A_{n} C_{n}\right\}_{n}$.

Another important algebraic property of a.c.s. is stated in the next theorem [49, Lemma 1].

THEOREM 2.37. Let $s \in \mathbb{N}$, let $\left\{A_{n}=\left[A_{n, i j}\right]_{i, j=1}^{s}\right\}_{n}$ and $\left\{B_{n}^{(m)}=\left[B_{n, i j}^{(m)}\right]_{i, j=1}^{s}\right\}_{n}$ be sequences of block matrices, and suppose that

$$
\left\{B_{n, i j}^{(m)}\right\}_{n} \stackrel{\text { a.c.s. }}{\longrightarrow}\left\{A_{n, i j}\right\}_{n}, \quad i, j=1, \ldots, s .
$$

Then $\left\{B_{n}^{(m)}\right\}_{n} \stackrel{\text { a.c.s. }}{\longrightarrow}\left\{A_{n}\right\}_{n}$.

2.5.5. A criterion to identify a.c.s. In practical applications, it often happens that a sequence of sequences of matrices $\left\{\left\{B_{n, m}\right\}_{n}\right\}_{m}$ is given together with another sequence of matrices $\left\{A_{n}\right\}_{n}$, and one would like to show that $\left\{B_{n, m}\right\}_{n} \stackrel{\text { a.c.s. }}{\longrightarrow}\left\{A_{n}\right\}_{n}$ without resorting to Definition 2.29. A way for solving this problem consists in choosing a suitable gauge 
function $\varphi$ and proving that $d_{\text {a.c.s. }}^{\varphi}\left(\left\{B_{n, m}\right\}_{n},\left\{A_{n}\right\}_{n}\right) \rightarrow 0$ as $m \rightarrow \infty$. Another criterion is provided in the next theorem [50, Corollary 5.3].

THEOREM 2.38. Let $\left\{A_{n}\right\}_{n},\left\{B_{n, m}\right\}_{n}$ be sequences of matrices with $A_{n}, B_{n, m}$ of size $d_{n}$, and let $1 \leq p<\infty$. Suppose that for every $m$ there exists $n_{m}$ such that, for $n \geq n_{m}$,

$$
\left\|A_{n}-B_{n, m}\right\|_{p}^{p} \leq \varepsilon(m, n) d_{n}
$$

where $\lim _{m \rightarrow \infty} \limsup _{n \rightarrow \infty} \varepsilon(m, n)=0$. Then $\left\{B_{n, m}\right\}_{n} \stackrel{\text { a.c.s. }}{\longrightarrow}\left\{A_{n}\right\}_{n}$.

2.6. Block Toeplitz matrices. A matrix of the form

$$
\left[A_{i-j}\right]_{i, j=1}^{n}=\left[\begin{array}{ccccc}
A_{0} & A_{-1} & \cdots & \cdots & A_{-(n-1)} \\
A_{1} & \ddots & \ddots & & \vdots \\
\vdots & \ddots & \ddots & \ddots & \vdots \\
\vdots & & \ddots & \ddots & A_{-1} \\
A_{n-1} & \cdots & \cdots & A_{1} & A_{0}
\end{array}\right] \in \mathbb{C}^{s n \times s n}
$$

with blocks $A_{k} \in \mathbb{C}^{s \times s}$, for $k=-(n-1), \ldots, n-1$, is called an $s$-block Toeplitz matrix. If $s=1$, it is simply referred to as a Toeplitz matrix. Given a function $f \in L^{1}([-\pi, \pi], s)$, its Fourier coefficients are denoted by

$$
f_{k}=\frac{1}{2 \pi} \int_{-\pi}^{\pi} f(\theta) e^{-\mathrm{i} k \theta} \mathrm{d} \theta \in \mathbb{C}^{s \times s}, \quad k \in \mathbb{Z},
$$

where the integrals are computed componentwise. The $n$th ( $s$-block) Toeplitz matrix associated with $f$ is defined as

$$
T_{n}(f)=\left[f_{i-j}\right]_{i, j=1}^{n} \in \mathbb{C}^{s n \times s n} .
$$

We call $\left\{T_{n}(f)\right\}_{n}$ the (s-block) Toeplitz sequence associated with $f$, which in turn is called the generating function of $\left\{T_{n}(f)\right\}_{n}$.

For each fixed $s, n \in \mathbb{N}$, the map $T_{n}(\cdot): L^{1}([-\pi, \pi], s) \rightarrow \mathbb{C}^{s n \times s n}$ is linear, i.e.,

$$
T_{n}(\alpha f+\beta g)=\alpha T_{n}(f)+\beta T_{n}(g), \quad \alpha, \beta \in \mathbb{C}, \quad f, g \in L^{1}([-\pi, \pi], s) .
$$

Moreover, it is clear from the definition that $T_{n}\left(I_{s}\right)=I_{s n}$. If $f \in L^{1}([-\pi, \pi], s)$, let $f^{*}$ be its conjugate transpose. It is not difficult to show that

$$
T_{n}(f)^{*}=T_{n}\left(f^{*}\right), \quad f \in L^{1}([-\pi, \pi], s), \quad s, n \in \mathbb{N} .
$$

In particular, if $f$ is Hermitian a.e., then the matrices $T_{n}(f)$ are Hermitian.

Theorem 2.39 is a fundamental result about block Toeplitz matrices. It provides the singular value distribution of block Toeplitz sequences generated by a matrix-valued function $f \in L^{1}([-\pi, \pi], s)$ and the spectral distribution of block Toeplitz sequences generated by a Hermitian matrix-valued function $f \in L^{1}([-\pi, \pi], s)$. For the eigenvalues it goes back to Szegó [59], and for the singular values it was established by Avram [2] and Parter [64]. They assumed that $f \in L^{\infty}([-\pi, \pi], s)$ and $s=1$; see [23, Section 5] and [24, Section 10.14] for more on the subject in the case of $L^{\infty}$ generating functions. The extension to $f \in L^{1}([-\pi, \pi], s)$ with $s=1$ was performed by Tyrtyshnikov and Zamarashkin [82, 83, 84], and the final generalization to $f \in L^{1}([-\pi, \pi], s)$ with $s \geq 1$ is due to Tilli [80]. We also refer the reader to [50, Section 6.5] for a proof of Theorem 2.39 based on the notion of approximating classes 
of sequences; the proof in [50, Section 6.5] is made only for $s=1$, but the argument is general and can be extended to matrix-valued generating functions.

THEOREM 2.39. If $f \in L^{1}([-\pi, \pi], s)$, then $\left\{T_{n}(f)\right\}_{n} \sim_{\sigma} f$. If moreover $f$ is Hermitian a.e., then $\left\{T_{n}(f)\right\}_{n} \sim_{\lambda} f$.

Important inequalities involving Toeplitz matrices and Schatten $p$-norms originally appeared in [77, Corollary 4.2]. They have been generalized to block Toeplitz matrices in [74, Corollary 3.5]. We report them in the next theorem for future use.

THEOREM 2.40. Let $f \in L^{p}([-\pi, \pi], s)$ and $n \in \mathbb{N}$. Then, using the natural convention $1 / \infty=0$, the inequality $\left\|T_{n}(f)\right\|_{p} \leq(n / 2 \pi)^{1 / p}\|f\|_{L^{p}}$ holds for all $p \in[1, \infty]$.

The next theorem shows that the product of block Toeplitz matrices generated by $L^{\infty}$ functions is "close" to the block Toeplitz matrix generated by the product of the generating functions. For the corresponding proof, see [55, Theorem 2.5].

THEOREM 2.41. Let $f_{i} \in L^{\infty}([-\pi, \pi], s)$, for $i=1, \ldots, q$. Then,

$$
\lim _{n \rightarrow \infty} \frac{\left\|\prod_{i=1}^{q} T_{n}\left(f_{i}\right)-T_{n}\left(\prod_{i=1}^{q} f_{i}\right)\right\|_{1}}{n}=0 .
$$

We end this section with a result highlighting the connection between block Toeplitz matrices and block matrices with block Toeplitz blocks. It generalizes [49, Lemma 3]. Recall that $\Pi_{n, s, r}$ denotes the special permutation matrix (2.1).

THEOREM 2.42. Let $n \in \mathbb{N}$, let $f_{i j}:[-\pi, \pi] \rightarrow \mathbb{C}^{r \times r}$ be in $L^{1}([-\pi, \pi], r)$, for $i, j=1, \ldots, s$, and set $f=\left[f_{i j}\right]_{i, j=1}^{s}$. The block matrix $T_{n}=\left[T_{n}\left(f_{i j}\right)\right]_{i, j=1}^{s}$ is similar via the permutation (2.1) to the block Toeplitz matrix $T_{n}(f)$, that is, $\Pi_{n, s, r} T_{n} \Pi_{n, s, r}^{T}=T_{n}(f)$.

Proof. Since $T_{n}=\sum_{i, j=1}^{s} E_{i j}^{(s)} \otimes T_{n}\left(f_{i j}\right)$ and $T_{n}(f)=\sum_{i, j=1}^{s} T_{n}\left(E_{i j}^{(s)} \otimes f_{i j}\right)$ by the linearity of the map $T_{n}(\cdot)$, it is enough to show that

$$
\Pi_{n, s, r}\left(E \otimes T_{n}(g)\right) \Pi_{n, s, r}^{T}=T_{n}(E \otimes g), \quad \forall g \in L^{1}([-\pi, \pi], r), \quad \forall E \in \mathbb{C}^{s \times s} .
$$

By the properties of tensor products (see Section 2.2.2),

$$
\begin{aligned}
\Pi_{n, s, r}\left(E \otimes T_{n}(g)\right) \Pi_{n, s, r}^{T} & \\
\quad & =\left[\sum_{k=1}^{n} \mathbf{e}_{k} \otimes I_{s} \otimes \mathbf{e}_{k}^{T} \otimes I_{r}\right]\left(E \otimes T_{n}(g)\right)\left[\sum_{\ell=1}^{n} \mathbf{e}_{\ell}^{T} \otimes I_{s} \otimes \mathbf{e}_{\ell} \otimes I_{r}\right] \\
& =\sum_{k, \ell=1}^{n}\left(\mathbf{e}_{k} \otimes I_{s} \otimes \mathbf{e}_{k}^{T} \otimes I_{r}\right)\left(E \otimes T_{n}(g)\right)\left(\mathbf{e}_{\ell}^{T} \otimes I_{s} \otimes \mathbf{e}_{\ell} \otimes I_{r}\right) \\
& =\sum_{k, \ell=1}^{n} \mathbf{e}_{k} \mathbf{e}_{\ell}^{T} \otimes E \otimes\left(\mathbf{e}_{k}^{T} \otimes I_{r}\right) T_{n}(g)\left(\mathbf{e}_{\ell} \otimes I_{r}\right) \\
& =\sum_{k, \ell=1}^{n} \mathbf{e}_{k} \mathbf{e}_{\ell}^{T} \otimes E \otimes g_{k-\ell}=\sum_{k, \ell=1}^{n} \mathbf{e}_{k} \mathbf{e}_{\ell}^{T} \otimes(E \otimes g)_{k-\ell}=T_{n}(E \otimes g),
\end{aligned}
$$

as required.

2.7. Block diagonal sampling matrices. If $n \in \mathbb{N}$ and $a:[0,1] \rightarrow \mathbb{C}^{s \times s}$, then we define the $s$-block diagonal sampling matrix $D_{n}(a)$ as the following block diagonal matrix of size $s n \times s n$ :

$$
D_{n}(a)=\operatorname{diag}_{i=1, \ldots, n} a\left(\frac{i}{n}\right)=\bigoplus_{i=1}^{n} a\left(\frac{i}{n}\right) .
$$




\section{ETNA}

Kent State University and

Johann Radon Institute (RICAM)

If $\left(\mathbb{C}^{s \times s}\right)^{[0,1]}$ denotes the space of all functions $a:[0,1] \rightarrow \mathbb{C}^{s \times s}$, then the map $D_{n}(\cdot):\left(\mathbb{C}^{s \times s}\right)^{[0,1]} \rightarrow \mathbb{C}^{s n \times s n}$ is linear, i.e.,

$$
D_{n}(\alpha a+\beta b)=\alpha D_{n}(a)+\beta D_{n}(b), \quad \alpha, \beta \in \mathbb{C}, \quad a, b \in\left(\mathbb{C}^{s \times s}\right)^{[0,1]} .
$$

Moreover, it is clear from the definition that $D_{n}(E)=T_{n}(E)$ for all constant matrices $E \in \mathbb{C}^{s \times s}$ and $D_{n}(a)^{*}=D_{n}\left(a^{*}\right)$ for all $a \in\left(\mathbb{C}^{s \times s}\right)^{[0,1]}$. The next result, which is the version of Theorem 2.42 for block diagonal sampling matrices, highlights the connection between block diagonal sampling matrices and block matrices with block diagonal sampling blocks. It is a generalization of [49, Lemma 4].

THEOREM 2.43. Let $n \in \mathbb{N}$, let $a_{i j}:[0,1] \rightarrow \mathbb{C}^{r \times r}$, for $i, j=1, \ldots, s$, and set $a=\left[a_{i j}\right]_{i, j=1}^{s}$. The block matrix $D_{n}=\left[D_{n}\left(a_{i j}\right)\right]_{i, j=1}^{s}$ is similar via the permutation (2.1) to the block diagonal sampling matrix $D_{n}(a)$, that is, $\Pi_{n, s, r} D_{n} \Pi_{n, s, r}^{T}=D_{n}(a)$.

Proof. With obvious adaptations, it is the same as the proof of Theorem 2.42.

Throughout this work, if $n, s \in \mathbb{N}$ and $a:[0,1] \rightarrow \mathbb{C}$, we denote by $D_{n, s}(a)$ the $s$-block diagonal sampling matrix given by

$$
D_{n, s}(a)=D_{n}\left(a I_{s}\right) .
$$

Note that $D_{n, 1}(a)=D_{n}(a)$ for every $a:[0,1] \rightarrow \mathbb{C}$.

3. Block locally Toeplitz sequences. The theory of (scalar) LT sequences dates back to Tilli's pioneering paper [79]. It was then carried forward in [75, 76], and it was finally developed in a systematic way in [50, Chapter 7] and [51, Chapter 4]. The theory of block LT sequences was originally suggested in [76, Section 3.3] and has been recently addressed in [55] in a systematic way. It should be said, however, that the approach followed in [55], besides being unnecessarily complicated, is not the "right" generalization to the block case of the theory of LT sequences presented in [50,51]. In particular, block LT sequences have been defined in [55] in terms of Hadamard products, which have now been discovered to be pointless. In this chapter, we develop the theory of block LT sequences by following the "right" approach, which allows us to considerably simplify the presentation with respect to [55] and to extend to the block case several results from $[50,51]$ that have been ignored in [55]. The topic is presented here on an abstract level, whereas for motivations and insights we refer the reader to Chapter 1; see also the introduction of Tilli's paper [79] and [50, Section 7.1].

3.1. The block LT operator. Similarly to the case of (scalar) LT sequences, the theory of block LT sequences begins with the definition of block LT operators. The definition given here is formulated only for scalar functions $a:[0,1] \rightarrow \mathbb{C}$. It is formally the same as the definition of (scalar) LT operator given in [50, Chapter 7], and it is much simpler than the one given in [55]; in particular, it does not involve any Hadamard product.

DEFINITION 3.1 (Block locally Toeplitz operator). Let $m, n, s \in \mathbb{N}$, let a $:[0,1] \rightarrow \mathbb{C}$, and let $f \in L^{1}([-\pi, \pi], s)$. The block locally Toeplitz (LT) operator is defined as the following sn $\times$ sn matrix:

$$
\begin{aligned}
L T_{n}^{m}(a, f) & =D_{m}(a) \otimes T_{\lfloor n / m\rfloor}(f) \oplus O_{s(n \bmod m)} \\
& =\underset{i=1, \ldots, m}{\operatorname{diag}}\left[a\left(\frac{i}{m}\right) T_{\lfloor n / m\rfloor}(f)\right] \oplus O_{s(n \bmod m)} \\
& =\bigoplus_{i=1}^{m} a\left(\frac{i}{m}\right) T_{\lfloor n / m\rfloor}(f) \oplus O_{s(n \bmod m) .}
\end{aligned}
$$

It is understood that $L T_{n}^{m}(a, f)=O_{s n}$ when $n<m$ and that the term $O_{s(n \bmod m)}$ is not present when $n$ is a multiple of $m$. Moreover, here and in what follows, the tensor product 
operation $\otimes$ is always applied before the direct sum $\oplus$, exactly as in the case of numbers, where multiplication is always applied before addition.

In this section we investigate the properties of the block LT operator. We first note that Definition 3.1 reduces to the definition of (scalar) LT operator [50, Definition 7.1] if $s=1$. Moreover, for every $m, n, s \in \mathbb{N}$, every $a, b:[0,1] \rightarrow \mathbb{C}$, every $f, g \in L^{1}([-\pi, \pi], s)$, and every $\alpha, \beta \in \mathbb{C}$, we have

$$
\begin{aligned}
L T_{n}^{m}(a, f)^{*} & =L T_{n}^{m}\left(\bar{a}, f^{*}\right), \\
L T_{n}^{m}(\alpha a+\beta b, f) & =\alpha L T_{n}^{m}(a, f)+\beta L T_{n}^{m}(b, f), \\
L T_{n}^{m}(a, \alpha f+\beta g) & =\alpha L T_{n}^{m}(a, f)+\beta L T_{n}^{m}(a, g), \\
\left\|L T_{n}^{m}(a, f)\right\|_{1} & =\left\|D_{m}(a)\right\|_{1}\left\|T_{\lfloor n / m\rfloor}(f)\right\|_{1} \leq \frac{1}{2 \pi} \sum_{i=1}^{m}\left|a\left(\frac{i}{m}\right)\right|\|f\|_{L^{1}}\lfloor n / m\rfloor,
\end{aligned}
$$

where in the last inequality we invoked Theorem 2.40 .

REMARK 3.2. Let $a:[0,1] \rightarrow \mathbb{C}$ be a bounded function, and take any sequence $\left\{f_{k}\right\}_{k} \subset L^{1}([-\pi, \pi], s)$ such that $f_{k} \rightarrow f$ in $L^{1}([-\pi, \pi], s)$. By (3.3) and (3.4), for every $k, n, m$, we have

$$
\left\|L T_{n}^{m}(a, f)-L T_{n}^{m}\left(a, f_{k}\right)\right\|_{1}=\left\|L T_{n}^{m}\left(a, f-f_{k}\right)\right\|_{1} \leq n\|a\|_{\infty}\left\|f-f_{k}\right\|_{L^{1}} .
$$

By Theorem 2.38, this implies that $\left\{L T_{n}^{m}\left(a, f_{k}\right)\right\}_{n} \stackrel{\text { a.c.s. }}{\longrightarrow}\left\{L T_{n}^{m}(a, f)\right\}_{n}$ as $k \rightarrow \infty$ for every $m \in \mathbb{N}$.

Proposition 3.3. Let $a_{i}:[0,1] \rightarrow \mathbb{C}$ be bounded, and let $f_{i} \in L^{\infty}([-\pi, \pi], s)$ for $i=1, \ldots, q$. Then, for every $n, m \in \mathbb{N}$,

$$
\left\|\prod_{i=1}^{q} L T_{n}^{m}\left(a_{i}, f_{i}\right)-L T_{n}^{m}\left(\prod_{i=1}^{q} a_{i}, \prod_{i=1}^{q} f_{i}\right)\right\|_{1} \leq \varepsilon(\lfloor n / m\rfloor) n,
$$

where

$$
\varepsilon(k)=\left\|\prod_{i=1}^{q} a_{i}\right\|_{\infty} \frac{\left\|\prod_{i=1}^{q} T_{k}\left(f_{i}\right)-T_{k}\left(\prod_{i=1}^{q} f_{i}\right)\right\|_{1}}{k}
$$

and $\lim _{k \rightarrow \infty} \varepsilon(k)=0$ by Theorem 2.41. In particular, for every $m \in \mathbb{N}$,

$$
d_{\text {a.c.s. }}\left(\left\{\prod_{i=1}^{q} L T_{n}^{m}\left(a_{i}, f_{i}\right)\right\}_{n},\left\{L T_{n}^{m}\left(\prod_{i=1}^{q} a_{i}, \prod_{i=1}^{q} f_{i}\right)\right\}_{n}\right)=0 .
$$

Proof. By the properties of tensor products and direct sums, we have

$$
\begin{aligned}
& \left\|\prod_{i=1}^{q} L T_{n}^{m}\left(a_{i}, f_{i}\right)-L T_{n}^{m}\left(\prod_{i=1}^{q} a_{i}, \prod_{i=1}^{q} f_{i}\right)\right\|_{1} \\
& \quad=\left\|D_{m}\left(\prod_{i=1}^{q} a_{i}\right) \otimes\left(\prod_{i=1}^{q} T_{\lfloor n / m\rfloor}\left(f_{i}\right)-T_{\lfloor n / m\rfloor}\left(\prod_{i=1}^{q} f_{i}\right)\right) \oplus O_{s(n \bmod m)}\right\|_{1} \\
& \quad=\left\|D_{m}\left(\prod_{i=1}^{q} a_{i}\right)\right\|\left\|\prod_{i=1}^{q} T_{\lfloor n / m\rfloor}\left(f_{i}\right)-T_{\lfloor n / m\rfloor}\left(\prod_{i=1}^{q} f_{i}\right)\right\|_{1} \\
& \quad \leq n\left\|\prod_{i=1}^{q} a_{i}\right\|_{\infty} \frac{\left\|\prod_{i=1}^{q} T_{\lfloor n / m\rfloor}\left(f_{i}\right)-T_{\lfloor n / m\rfloor}\left(\prod_{i=1}^{q} f_{i}\right)\right\|_{1}}{\lfloor n / m\rfloor} .
\end{aligned}
$$


This proves (3.5). Since $\varepsilon(k) \rightarrow 0$ as $k \rightarrow \infty$ by Theorem 2.41, equation (3.6) follows immediately from (3.5) and Theorems 2.16 and 2.30.

THEOREM 3.4. Suppose that $a^{(i, j)}:[0,1] \rightarrow \mathbb{C}$ is Riemann-integrable and that $f^{(i, j)} \in L^{\infty}([-\pi, \pi], s)$, for $i=1, \ldots, p$ and $j=1, \ldots, q_{i}$. Then, for every $m \in \mathbb{N}$,

$$
\left\{\sum_{i=1}^{p} \prod_{j=1}^{q_{i}} L T_{n}^{m}\left(a^{(i, j)}, f^{(i, j)}\right)\right\}_{n} \sim_{\sigma} \sum_{i=1}^{p} \prod_{j=1}^{q_{i}} a_{m}^{(i, j)}(x) f^{(i, j)}(\theta)
$$

where

$$
a_{m}^{(i, j)}(x)=\sum_{k=1}^{m} a^{(i, j)}\left(\frac{k}{m}\right) \chi_{\left[\frac{k-1}{m}, \frac{k}{m}\right)}(x) .
$$

Proof. By Proposition 3.3 and Remark 2.35, it is enough to show that

$$
\left\{\sum_{i=1}^{p} L T_{n}^{m}\left(\prod_{j=1}^{q_{i}} a^{(i, j)}, \prod_{j=1}^{q_{i}} f^{(i, j)}\right)\right\}_{n} \sim_{\sigma} \sum_{i=1}^{p} \prod_{j=1}^{q_{i}} a_{m}^{(i, j)}(x) f^{(i, j)}(\theta) .
$$

Note that

$$
\begin{aligned}
& \sum_{i=1}^{p} L T_{n}^{m}\left(\prod_{j=1}^{q_{i}} a^{(i, j)}, \prod_{j=1}^{q_{i}} f^{(i, j)}\right) \\
& =\left(\sum_{i=1}^{p} D_{m}\left(\prod_{j=1}^{q_{i}} a^{(i, j)}\right) \otimes T_{\lfloor n / m\rfloor}\left(\prod_{j=1}^{q_{i}} f^{(i, j)}\right)\right) \oplus O_{s(n \bmod m) .}
\end{aligned}
$$

Recalling (2.17), for every $k=1, \ldots, m$, the $k$ th diagonal block of size $s\lfloor n / m\rfloor$ of the matrix (3.8) is given by

$$
\sum_{i=1}^{p}\left(\prod_{j=1}^{q_{i}} a^{(i, j)}\left(\frac{k}{m}\right)\right) T_{\lfloor n / m\rfloor}\left(\prod_{j=1}^{q_{i}} f^{(i, j)}\right)=T_{\lfloor n / m\rfloor}\left(\sum_{i=1}^{p} \prod_{j=1}^{q_{i}} a^{(i, j)}\left(\frac{k}{m}\right) f^{(i, j)}\right) .
$$

It follows that the singular values of the matrix (3.8) are

$$
\sigma_{\ell}\left(T_{\lfloor n / m\rfloor}\left(\sum_{i=1}^{p} \prod_{j=1}^{q_{i}} a^{(i, j)}\left(\frac{k}{m}\right) f^{(i, j)}\right)\right), \quad \ell=1, \ldots, s\left\lfloor\frac{n}{m}\right\rfloor, \quad k=1, \ldots, m,
$$

plus further $s(n \bmod m)=o(n)$ singular values which are equal to 0 . Therefore, by Theorem 2.39, since $\sum_{i=1}^{p} \prod_{j=1}^{q_{i}} a^{(i, j)}\left(\frac{k}{m}\right) f^{(i, j)} \in L^{\infty}([-\pi, \pi], s)$, for any $F \in C_{c}(\mathbb{R})$, we have

$$
\begin{aligned}
& \lim _{n \rightarrow \infty} \frac{1}{s n} \sum_{r=1}^{s n} F\left(\sigma_{r}\left(\sum_{i=1}^{p} L T_{n}^{m}\left(\prod_{j=1}^{q_{i}} a^{(i, j)}, \prod_{j=1}^{q_{i}} f^{(i, j)}\right)\right)\right) \\
& =\lim _{n \rightarrow \infty} \frac{m s\lfloor n / m\rfloor}{s n} \frac{1}{m} \sum_{k=1}^{m} \frac{1}{s\lfloor n / m\rfloor} \sum_{\ell=1}^{s\lfloor n / m\rfloor} F\left(\sigma_{\ell}\left(T_{\lfloor n / m\rfloor}\left(\sum_{i=1}^{p} \prod_{j=1}^{q_{i}} a^{(i, j)}\left(\frac{k}{m}\right) f^{(i, j)}\right)\right)\right) \\
& =\frac{1}{m} \sum_{k=1}^{m} \frac{1}{2 \pi} \int_{-\pi}^{\pi} \frac{1}{s} \sum_{\ell=1}^{s} F\left(\sigma_{\ell}\left(\sum_{i=1}^{p} \prod_{j=1}^{q_{i}} a^{(i, j)}\left(\frac{k}{m}\right) f^{(i, j)}(\theta)\right)\right) \mathrm{d} \theta
\end{aligned}
$$




$$
=\frac{1}{2 \pi} \int_{0}^{1} \int_{-\pi}^{\pi} \frac{1}{s} \sum_{\ell=1}^{s} F\left(\sigma_{\ell}\left(\sum_{i=1}^{p} \prod_{j=1}^{q_{i}} a_{m}^{(i, j)}(x) f^{(i, j)}(\theta)\right)\right) \mathrm{d} \theta \mathrm{d} x .
$$

This concludes the proof.

THEOREM 3.5. Suppose that $a^{(i, j)}:[0,1] \rightarrow \mathbb{C}$ is Riemann-integrable and that $f^{(i, j)} \in L^{\infty}([-\pi, \pi], s)$, for $i=1, \ldots, p$ and $j=1, \ldots, q_{i}$. Then, for every $m \in \mathbb{N}$,

$$
\left\{\Re\left(\sum_{i=1}^{p} \prod_{j=1}^{q_{i}} L T_{n}^{m}\left(a^{(i, j)}, f^{(i, j)}\right)\right)\right\}_{n} \sim_{\lambda} \Re\left(\sum_{i=1}^{p} \prod_{j=1}^{q_{i}} a_{m}^{(i, j)}(x) f^{(i, j)}(\theta)\right),
$$

where $a_{m}^{(i, j)}$ is defined in (3.7).

Proof. The proof follows the same pattern as the proof of Theorem 3.4. By Proposition 3.3 and Remark 2.35, it is enough to show that

$$
\left\{\Re\left(\sum_{i=1}^{p} L T_{n}^{m}\left(\prod_{j=1}^{q_{i}} a^{(i, j)}, \prod_{j=1}^{q_{i}} f^{(i, j)}\right)\right)\right\}_{n} \sim_{\lambda} \Re\left(\sum_{i=1}^{p} \prod_{j=1}^{q_{i}} a_{m}^{(i, j)}(x) f^{(i, j)}(\theta)\right) .
$$

Note that

$$
\begin{aligned}
& \Re\left(\sum_{i=1}^{p} L T_{n}^{m}\left(\prod_{j=1}^{q_{i}} a^{(i, j)}, \prod_{j=1}^{q_{i}} f^{(i, j)}\right)\right) \\
& =\Re\left(\sum_{i=1}^{p} D_{m}\left(\prod_{j=1}^{q_{i}} a^{(i, j)}\right) \otimes T_{\lfloor n / m\rfloor}\left(\prod_{j=1}^{q_{i}} f^{(i, j)}\right)\right) \oplus O_{s(n \bmod m)} .
\end{aligned}
$$

Recalling (2.17) and (2.18), for every $k=1, \ldots, m$, the $k$ th diagonal block of size $s\lfloor n / m\rfloor$ of the matrix (3.10) is given by

$\Re\left(\sum_{i=1}^{p}\left(\prod_{j=1}^{q_{i}} a^{(i, j)}\left(\frac{k}{m}\right)\right) T_{\lfloor n / m\rfloor}\left(\prod_{j=1}^{q_{i}} f^{(i, j)}\right)\right)=T_{\lfloor n / m\rfloor}\left(\Re\left(\sum_{i=1}^{p} \prod_{j=1}^{q_{i}} a^{(i, j)}\left(\frac{k}{m}\right) f^{(i, j)}\right)\right)$.

It follows that the eigenvalues of the matrix (3.10) are

$$
\lambda_{\ell}\left(T_{\lfloor n / m\rfloor}\left(\Re\left(\sum_{i=1}^{p} \prod_{j=1}^{q_{i}} a^{(i, j)}\left(\frac{k}{m}\right) f^{(i, j)}\right)\right)\right), \quad \ell=1, \ldots, s\left\lfloor\frac{n}{m}\right\rfloor, \quad k=1, \ldots, m,
$$

plus further $s(n \bmod m)=o(n)$ eigenvalues which are equal to 0 . Therefore, by Theorem 2.39, since $\Re\left(\sum_{i=1}^{p} \prod_{j=1}^{q_{i}} a^{(i, j)}\left(\frac{k}{m}\right) f^{(i, j)}\right) \in L^{\infty}([-\pi, \pi], s)$, following the same derivation as in (3.9), we obtain, for any $F \in C_{c}(\mathbb{C})$,

$$
\begin{aligned}
& \lim _{n \rightarrow \infty} \frac{1}{s n} \sum_{r=1}^{s n} F\left(\lambda_{r}\left(\Re\left(\sum_{i=1}^{p} L T_{n}^{m}\left(\prod_{j=1}^{q_{i}} a^{(i, j)}, \prod_{j=1}^{q_{i}} f^{(i, j)}\right)\right)\right)\right) \\
& =\frac{1}{2 \pi} \int_{0}^{1} \int_{-\pi}^{\pi} \frac{1}{s} \sum_{\ell=1}^{s} F\left(\lambda_{\ell}\left(\Re\left(\sum_{i=1}^{p} \prod_{j=1}^{q_{i}} a_{m}^{(i, j)}(x) f^{(i, j)}(\theta)\right)\right)\right) \mathrm{d} \theta \mathrm{d} x .
\end{aligned}
$$

This concludes the proof.

PRoposition 3.6. Let $a:[0,1] \rightarrow \mathbb{C}$ be a Riemann-integrable function, and let $f \in L^{1}([-\pi, \pi], s)$. Then, for every $m \in \mathbb{N}$,

$$
\left\{L T_{n}^{m}(a, f)\right\}_{n} \sim_{\sigma} a_{m}(x) f(\theta),
$$


where

$$
a_{m}(x)=\sum_{k=1}^{m} a\left(\frac{k}{m}\right) \chi_{\left[\frac{k-1}{m}, \frac{k}{m}\right)}(x) .
$$

Proof. Fix $m \in \mathbb{N}$, and take any sequence $\left\{f_{k}\right\}_{k} \subset L^{\infty}([-\pi, \pi], s)$ such that $f_{k} \rightarrow f$ a.e. and in $L^{1}([-\pi, \pi], s)$. We have:

- $\left\{L T_{n}^{m}\left(a, f_{k}\right)\right\}_{n} \stackrel{\text { a.c.s. }}{\longrightarrow}\left\{L T_{n}^{m}(a, f)\right\}_{n}$ by Remark 3.2;

- $\left\{L T_{n}^{m}\left(a, f_{k}\right)\right\}_{n} \sim_{\sigma} a_{m}(x) f_{k}(\theta)$ by Theorem 3.4;

- $a_{m}(x) f_{k}(\theta) \rightarrow a_{m}(x) f(\theta)$ a.e. (and hence also in measure).

We conclude that $\left\{L T_{n}^{m}(a, f)\right\}_{n} \sim_{\sigma} a_{m}(x) f(\theta)$ by Theorem 2.33.

3.2. Definition of block LT sequences. The notion of block LT sequences is formalized in the next definition.

DEFINITION 3.7 (Block locally Toeplitz sequence). Let $\left\{A_{n}\right\}_{n}$ be an s-block matrixsequence, let $a:[0,1] \rightarrow \mathbb{C}$ be Riemann-integrable, and $f \in L^{1}([-\pi, \pi], s)$. We say that $\left\{A_{n}\right\}_{n}$ is an (s-block) locally Toeplitz (LT) sequence with symbol $a(x) f(\theta)$, and we write $\left\{A_{n}\right\}_{n} \sim_{\mathrm{LT}} a(x) f(\theta)$, if $\left\{L T_{n}^{m}(a, f)\right\}_{n} \stackrel{\text { a.c.s. }}{\longrightarrow}\left\{A_{n}\right\}_{n}$.

In what follows, unless specified otherwise, whenever we write a relation such as $\left\{A_{n}\right\}_{n} \sim_{\mathrm{LT}} a(x) f(\theta)$, it is understood that $\left\{A_{n}\right\}_{n}$ is an $s$-block matrix-sequence, that $a:[0,1] \rightarrow \mathbb{C}$ is Riemann-integrable, and that $f \in L^{1}([-\pi, \pi], s)$, as in Definition 3.7. Note that Definition 3.7 reduces to the definition of (scalar) LT sequences [50, Definition 7.2] if $s=1$. LT sequences are then special cases of block LT sequences.

3.3. Fundamental examples of block LT sequences. In this section we provide three fundamental examples of block LT sequences: zero-distributed sequences, sequences of block diagonal sampling matrices, and block Toeplitz sequences. These may be regarded as the "building blocks" of the theory of block GLT sequences because from them we can construct through algebraic operations a lot of other matrix-sequences which will turn out to be block GLT sequences.

3.3.1. Zero-distributed sequences. We show that any zero-distributed sequence is a block LT sequence with symbol given by the zero matrix.

THEOREM 3.8. Let $\left\{Z_{n}\right\}_{n}$ be an s-block matrix-sequence. The following are equivalent. 1. $\left\{Z_{n}\right\}_{n} \sim_{\sigma} 0$.

2. $\left\{O_{s n}\right\}_{n} \stackrel{\text { a.c.s. }}{\longrightarrow}\left\{Z_{n}\right\}_{n}$.

3. $\left\{Z_{n}\right\}_{n} \sim_{\mathrm{LT}} O_{s}$.

Proof. $(1 \Longleftrightarrow 2)$ By Theorem 2.30, we have $\left\{O_{s n}\right\}_{n} \stackrel{\text { a.c.s. }}{\longrightarrow}\left\{Z_{n}\right\}_{n}$ if and only if $d_{\text {a.c.s. }}\left(\left\{O_{s n}\right\}_{n},\left\{Z_{n}\right\}_{n}\right)=0$ if and only if $\left\{Z_{n}\right\}_{n} \sim_{\sigma} 0$.

$(2 \Longleftrightarrow 3)$ This equivalence follows from Definition 3.7 and the observation that $L T_{n}^{m}\left(0, O_{s}\right)=O_{s n}$ and $0 O_{s}=O_{s}$.

3.3.2. Sequences of block diagonal sampling matrices. We are going to see in Theorem 3.11 that $\left\{D_{n, s}(a)\right\}_{n}=\left\{D_{n}\left(a I_{s}\right)\right\}_{n} \sim_{\text {LT }} a(x) I_{s}$ whenever $a:[0,1] \rightarrow \mathbb{C}$ is Riemannintegrable. To prove Theorem 3.11 we shall need the following lemmas; cf. [50, Lemmas 5.6 and 7.1].

LeMma 3.9. Let $C$ be an $\ell \times \ell$ matrix and suppose that

$$
\|C\|_{p}^{p} \leq \varepsilon \ell^{\prime},
$$

where $p \in[1, \infty), \varepsilon \geq 0$, and $\ell^{\prime} \leq \ell$. Then we can write $C$ in the form

$$
C=R+N, \quad \operatorname{rank}(R) \leq \varepsilon^{\frac{1}{p+1}} \ell^{\prime}, \quad\|N\| \leq \varepsilon^{\frac{1}{p+1}} .
$$


LEMMA 3.10. For every $m \in \mathbb{N}$, let $\{x(m, k)\}_{k}$ be a sequence of numbers such that $x(m, k) \rightarrow x(m)$ as $k \rightarrow \infty$ and $x(m) \rightarrow 0$ as $m \rightarrow \infty$. Then, there exists a sequence $\{m(k)\}_{k} \subseteq \mathbb{N}$ such that $m(k) \rightarrow \infty$ and $x(m(k), k) \rightarrow 0$.

THEOREM 3.11. If $a:[0,1] \rightarrow \mathbb{C}$ is Riemann-integrable, then $\left\{D_{n, s}(a)\right\}_{n} \sim_{\mathrm{LT}} a(x) I_{s}$.

Proof. The proof consists of two steps. We first show that the thesis holds if $a$ is continuous. Then, by using an approximation argument, we show that it holds even in the case where $a$ is only supposed to be Riemann-integrable.

Step 1 . We prove that if $a \in C([0,1])$ and $\omega_{a}(\cdot)$ is the modulus of continuity of $a$, then

$$
\begin{aligned}
D_{n, s}(a) & =L T_{n}^{m}\left(a, I_{s}\right)+R_{n, m}+N_{n, m}, \\
\operatorname{rank}\left(R_{n, m}\right) & \leq s m, \quad\left\|N_{n, m}\right\| \leq \omega_{a}\left(\frac{1}{m}+\frac{m}{n}\right) .
\end{aligned}
$$

Since $\omega_{a}(\delta) \rightarrow 0$ as $\delta \rightarrow 0$, the convergence $\left\{L T_{n}^{m}\left(a, I_{s}\right)\right\}_{n} \stackrel{\text { a.c.s. }}{\longrightarrow}\left\{D_{n, s}(a)\right\}_{n}$ (and hence the relation $\left\{D_{n, s}(a)\right\}_{n} \sim_{\mathrm{LT}} a(x) I_{s}$ ) follows immediately from Definition 2.29 (take $n_{m}=m^{2}$, $\left.c(m)=1 / m, \omega(m)=\omega_{a}(2 / m)\right)$.

The matrix $L T_{n}^{m}\left(a, I_{s}\right)$ is the $s n \times s n$ block diagonal matrix given by

$$
L T_{n}^{m}\left(a, I_{s}\right)=D_{m}(a) \otimes I_{s\lfloor n / m\rfloor} \oplus O_{s(n \bmod m)} .
$$

For $i=1, \ldots, m\lfloor n / m\rfloor$, let $k=k(i)$ be the index in $\{1, \ldots, m\}$ such that

$$
(k-1)\lfloor n / m\rfloor+1 \leq i \leq k\lfloor n / m\rfloor .
$$

In other words, $k$ is the index such that the $i$ th $s \times s$ diagonal block of $L T_{n}^{m}\left(a, I_{s}\right)$ is given by $\left(L T_{n}^{m}\left(a, I_{s}\right)\right)_{i i}=a(k / m) I_{s}$. Taking into account that the $i$ th $s \times s$ diagonal block of $D_{n, s}(a)$ is given by $\left(D_{n, s}(a)\right)_{i i}=a(i / n) I_{s}$, for every $i=1, \ldots, m\lfloor n / m\rfloor$, we obtain

$$
\begin{aligned}
\left\|\left(L T_{n}^{m}\left(a, I_{s}\right)\right)_{i i}-\left(D_{n, s}(a)\right)_{i i}\right\| & =\left\|a\left(\frac{k}{m}\right) I_{s}-a\left(\frac{i}{n}\right) I_{s}\right\| \\
& =\left|a\left(\frac{k}{m}\right)-a\left(\frac{i}{n}\right)\right| \leq \omega_{a}\left(\frac{1}{m}+\frac{m}{n}\right),
\end{aligned}
$$

where the last inequality follows from the fact that

$$
\left|\frac{k}{m}-\frac{i}{n}\right| \leq \frac{k}{m}-\frac{(k-1)\lfloor n / m\rfloor}{n} \leq \frac{k}{m}-\frac{(k-1)(n / m-1)}{n}=\frac{1}{m}+\frac{k-1}{n} \leq \frac{1}{m}+\frac{m}{n} .
$$

Therefore, if $\tilde{D}_{n, s, m}(a)$ and $\hat{D}_{n, s, m}(a)$ are the $s n \times s n$ block diagonal matrices whose $i$ th $s \times s$ diagonal blocks $\left(\tilde{D}_{n, s, m}(a)\right)_{i i}$ and $\left(\hat{D}_{n, s, m}(a)\right)_{i i}$ are defined by

$$
\begin{aligned}
& \left(\tilde{D}_{n, s, m}(a)\right)_{i i}= \begin{cases}a(i / n) I_{s}, & \text { if } i \leq m\lfloor n / m\rfloor, \\
O_{s}, & \text { otherwise, }\end{cases} \\
& \left(\hat{D}_{n, s, m}(a)\right)_{i i}= \begin{cases}a(i / n) I_{s}, & \text { if } i>m\lfloor n / m\rfloor, \\
O_{s}, & \text { otherwise, }\end{cases}
\end{aligned}
$$

then $D_{n, s}(a)=\tilde{D}_{n, s, m}(a)+\hat{D}_{n, s, m}(a)$ and

$$
D_{n, s}(a)-L T_{n}^{m}\left(a, I_{s}\right)=\hat{D}_{n, s, m}(a)+\tilde{D}_{n, s, m}(a)-L T_{n}^{m}\left(a, I_{s}\right)=R_{n, m}+N_{n, m},
$$


where $R_{n, m}=\hat{D}_{n, s, m}(a)$ and $N_{n, m}=\tilde{D}_{n, s, m}(a)-L T_{n}^{m}\left(a, I_{s}\right)$ satisfy

$$
\begin{aligned}
\operatorname{rank}\left(R_{n, m}\right) & \leq s(n \bmod m)<s m, \\
\left\|N_{n, m}\right\| & =\max _{i=1, \ldots, m\lfloor n / m\rfloor}\left\|\left(L T_{n}^{m}\left(a, I_{s}\right)\right)_{i i}-\left(D_{n, s}(a)\right)_{i i}\right\| \leq \omega_{a}\left(\frac{1}{m}+\frac{m}{n}\right) .
\end{aligned}
$$

This completes the proof of (3.11).

Step 2. Let $a:[0,1] \rightarrow \mathbb{C}$ be any Riemann-integrable function. Take any sequence of continuous functions $a_{m}:[0,1] \rightarrow \mathbb{C}$ such that $a_{m} \rightarrow a$ in $L^{1}([0,1])$. By Step 1, $\left\{D_{n, s}\left(a_{m}\right)\right\}_{n} \sim_{\mathrm{LT}} a_{m}(x) I_{s}$. Hence, $\left\{L T_{n}^{k}\left(a_{m}, I_{s}\right)\right\}_{n} \stackrel{\text { a.c.s. }}{\longrightarrow}\left\{D_{n, s}\left(a_{m}\right)\right\}_{n}$ as $k \rightarrow \infty$, i.e., for every $m, k$ there is $n_{m, k}$ such that, for $n \geq n_{m, k}$,

$$
\begin{aligned}
D_{n, s}\left(a_{m}\right) & =L T_{n}^{k}\left(a_{m}, I_{s}\right)+R_{n, m, k}+N_{n, m, k}, \\
\operatorname{rank}\left(R_{n, m, k}\right) & \leq c(m, k) n, \quad\left\|N_{n, m, k}\right\| \leq \omega(m, k),
\end{aligned}
$$

where $\lim _{k \rightarrow \infty} c(m, k)=\lim _{k \rightarrow \infty} \omega(m, k)=0$. Moreover, we have the convergence $\left\{D_{n, s}\left(a_{m}\right)\right\}_{n} \stackrel{\text { a.c.s. }}{\longrightarrow}\left\{D_{n, s}(a)\right\}_{n}$. Indeed,

$$
\left\|D_{n, s}(a)-D_{n, s}\left(a_{m}\right)\right\|_{1}=s \sum_{j=1}^{n}\left|a\left(\frac{j}{n}\right)-a_{m}\left(\frac{j}{n}\right)\right|=\varepsilon(m, n) n,
$$

where

$$
\varepsilon(m, n)=\frac{s}{n} \sum_{j=1}^{n}\left|a\left(\frac{j}{n}\right)-a_{m}\left(\frac{j}{n}\right)\right| .
$$

By the Riemann-integrability of $\left|a-a_{m}\right|$ and the fact that $a_{m} \rightarrow a$ in $L^{1}([0,1])$, the quantity $\varepsilon(m, n)$ satisfies

$$
\lim _{m \rightarrow \infty} \lim _{n \rightarrow \infty} \varepsilon(m, n)=s \lim _{m \rightarrow \infty} \int_{0}^{1}\left|a(x)-a_{m}(x)\right| \mathrm{d} x=s \lim _{m \rightarrow \infty}\left\|a-a_{m}\right\|_{L^{1}}=0 .
$$

By Theorem 2.38, this implies that $\left\{D_{n, s}\left(a_{m}\right)\right\}_{n} \stackrel{\text { a.c.s. }}{\longrightarrow}\left\{D_{n, s}(a)\right\}_{n}$. Thus, for every $m$ there exists $n_{m}$ such that, for $n \geq n_{m}$,

$$
\begin{aligned}
D_{n, s}(a) & =D_{n, s}\left(a_{m}\right)+R_{n, m}+N_{n, m}, \\
\operatorname{rank}\left(R_{n, m}\right) & \leq c(m) n, \quad\left\|N_{n, m}\right\| \leq \omega(m),
\end{aligned}
$$

where $\lim _{m \rightarrow \infty} c(m)=\lim _{m \rightarrow \infty} \omega(m)=0$. It follows that, for every $m, k$ and every $n \geq \max \left(n_{m}, n_{m, k}\right)$,

$$
\begin{aligned}
& D_{n, s}(a)=L T_{n}^{k}\left(a, I_{s}\right)+\left[L T_{n}^{k}\left(a_{m}, I_{s}\right)-L T_{n}^{k}\left(a, I_{s}\right)\right] \\
& +\left(R_{n, m}+R_{n, m, k}\right)+\left(N_{n, m}+N_{n, m, k}\right), \\
& \operatorname{rank}\left(R_{n, m}+R_{n, m, k}\right) \leq(c(m)+c(m, k)) n, \\
& \left\|N_{n, m}+N_{n, m, k}\right\| \leq \omega(m)+\omega(m, k), \\
& \left\|L T_{n}^{k}\left(a_{m}, I_{s}\right)-L T_{n}^{k}\left(a, I_{s}\right)\right\|_{1} \leq \frac{s n}{k} \sum_{j=1}^{k}\left|a\left(\frac{j}{k}\right)-a_{m}\left(\frac{j}{k}\right)\right|=\varepsilon(m, k) n,
\end{aligned}
$$


where the last inequality follows from (3.2)-(3.4) and $\varepsilon(m, k)$ is defined as in (3.12) with " $n$ " replaced by " $k$ ". Let $\{m(k)\}_{k}$ be a sequence such that $m(k) \rightarrow \infty$ and

$$
\lim _{k \rightarrow \infty} \varepsilon(m(k), k)=\lim _{k \rightarrow \infty} c(m(k), k)=\lim _{k \rightarrow \infty} \omega(m(k), k)=0 .
$$

Note that such a sequence exists by Lemma 3.10 (apply the lemma with $x(m, k)=\varepsilon(m, k)+$ $c(m, k)+\omega(m, k))$. Then, for every $k$ and every $n \geq \max \left(n_{m(k)}, n_{m(k), k}\right)$,

$$
\begin{aligned}
& D_{n, s}(a)=L T_{n}^{k}\left(a, I_{s}\right)+\left[L T_{n}^{k}\left(a_{m(k)}, I_{s}\right)-L T_{n}^{k}\left(a, I_{s}\right)\right] \\
& +\left(R_{n, m(k)}+R_{n, m(k), k}\right)+\left(N_{n, m(k)}+N_{n, m(k), k}\right), \\
& \operatorname{rank}\left(R_{n, m(k)}+R_{n, m(k), k}\right) \leq(c(m(k))+c(m(k), k)) n, \\
& \left\|N_{n, m(k)}+N_{n, m(k), k}\right\| \leq \omega(m(k))+\omega(m(k), k), \\
& \left\|L T_{n}^{k}\left(a_{m(k)}, I_{s}\right)-L T_{n}^{k}\left(a, I_{s}\right)\right\|_{1} \leq \varepsilon(m(k), k) n .
\end{aligned}
$$

The application of Lemma 3.9 allows one to decompose $L T_{n}^{k}\left(a_{m(k)}, I_{s}\right)-L T_{n}^{k}\left(a, I_{s}\right)$ as the sum of a small-rank term $\hat{R}_{n, k}$, with the rank bounded by $\sqrt{\varepsilon(m(k), k)} n$, plus a smallnorm term $\hat{N}_{n, k}$ with norm bounded by $\sqrt{\varepsilon(m(k), k)}$. This shows that $\left\{L T_{n}^{k}\left(a, I_{s}\right)\right\}_{n} \stackrel{\text { a.c.s. }}{\longrightarrow}$ $\left\{D_{n, s}(a)\right\}_{n}$, hence $\left\{D_{n, s}(a)\right\}_{n} \sim_{\mathrm{LT}} a(x) I_{s}$.

\subsubsection{Block Toeplitz sequences.}

THEOREM 3.12. If $f \in L^{1}([-\pi, \pi], s)$, then $\left\{T_{n}(f)\right\}_{n} \sim_{\mathrm{LT}} f(\theta)$.

Proof. The proof consists of two steps. We first show that the thesis holds if $f$ is a matrix-valued trigonometric polynomial. Then, by using an approximation argument, we prove the theorem under the sole assumption that $f \in L^{1}([-\pi, \pi], s)$.

Step 1 . We show that if $f$ is a matrix-valued trigonometric polynomial of degree $q$, then

$$
T_{n}(f)=L T_{n}^{m}(1, f)+R_{n, m}, \quad \operatorname{rank}\left(R_{n, m}\right) \leq s(2 q+1) m .
$$

Once this is done, the convergence $\left\{L T_{n}^{m}(1, f)\right\}_{n} \stackrel{\text { a.c.s. }}{\longrightarrow}\left\{T_{n}(f)\right\}_{n}$ (and hence the relation $\left\{T_{n}(f)\right\}_{n} \sim_{\mathrm{LT}} f(\theta)$ ) follows immediately from Definition 2.29 (take $n_{m}=m^{2}$, $c(m)=(2 q+1) / m, \omega(m)=0)$.

Since $f$ has degree $q$, we can write $f(\theta)=\sum_{j=-q}^{q} f_{j} e^{\mathrm{i} j \theta}$. Moreover, the matrix $L T_{n}^{m}(1, f)$ is given by

$$
L T_{n}^{m}(1, f)=I_{m} \otimes T_{\lfloor n / m\rfloor}(f) \oplus O_{s(n \bmod m)} .
$$

A direct comparison between the matrix $T_{n}(f)$ and the matrix $L T_{n}^{m}(1, f)$ shows that if $n / m \geq 2 q+1$, then the number of nonzero rows of the difference $T_{n}(f)-L T_{n}^{m}(1, f)$ is at most $s(2 q m-q+(n \bmod m))$. Hence, if $n / m \geq 2 q+1$,

$$
T_{n}(f)=L T_{n}^{m}(1, f)+R_{n, m}, \quad \operatorname{rank}\left(R_{n, m}\right) \leq s(2 q m-q+(n \bmod m)) \leq s(2 q+1) m .
$$

This completes the proof of (3.13) for $n / m \geq 2 q+1$, but it is clear that (3.13) holds even if $n / m<2 q+1$ because in this case $s(2 q+1) m$ is greater than the matrix size $s n$.

Step 2. Let $f \in L^{1}([-\pi, \pi], s)$. Since the set of trigonometric polynomials is dense in $L^{1}([-\pi, \pi])$ (see, e.g., [50, Lemma 2.2]), there is a sequence of matrix-valued trigonometric polynomials $f_{m}:[-\pi, \pi] \rightarrow \mathbb{C}^{s \times s}$ such that $f_{m} \rightarrow f$ in $L^{1}([-\pi, \pi], s)$. By Step 1, $\left\{T_{n}\left(f_{m}\right)\right\}_{n} \sim_{\text {LT }} f_{m}(\theta)$. Hence, $\left\{L T_{n}^{k}\left(1, f_{m}\right)\right\}_{n} \stackrel{\text { a.c.s. }}{\longrightarrow}\left\{T_{n}\left(f_{m}\right)\right\}_{n}$ as $k \rightarrow \infty$, i.e., for every $m, k$ there is $n_{m, k}$ such that, for $n \geq n_{m, k}$,

$$
\begin{aligned}
T_{n}\left(f_{m}\right) & =L T_{n}^{k}\left(1, f_{m}\right)+R_{n, m, k}+N_{n, m, k}, \\
\operatorname{rank}\left(R_{n, m, k}\right) & \leq c(m, k) n, \quad\left\|N_{n, m, k}\right\| \leq \omega(m, k),
\end{aligned}
$$


where $\lim _{k \rightarrow \infty} c(m, k)=\lim _{k \rightarrow \infty} \omega(m, k)=0$. Moreover, by Theorem 2.40,

$$
\left\|T_{n}(f)-T_{n}\left(f_{m}\right)\right\|_{1}=\left\|T_{n}\left(f-f_{m}\right)\right\|_{1} \leq n\left\|f-f_{m}\right\|_{L^{1}},
$$

and so $\left\{T_{n}\left(f_{m}\right)\right\}_{n} \stackrel{\text { a.c.s. }}{\longrightarrow}\left\{T_{n}(f)\right\}_{n}$ by Theorem 2.38. Thus, for every $m$ there exists $n_{m}$ such that, for $n \geq n_{m}$,

$$
\begin{aligned}
T_{n}(f) & =T_{n}\left(f_{m}\right)+R_{n, m}+N_{n, m}, \\
\operatorname{rank}\left(R_{n, m}\right) & \leq c(m) n, \quad\left\|N_{n, m}\right\| \leq \omega(m),
\end{aligned}
$$

where $\lim _{m \rightarrow \infty} c(m)=\lim _{m \rightarrow \infty} \omega(m)=0$. It follows that, for every $m, k$ and every $n \geq \max \left(n_{m}, n_{m, k}\right)$,

$$
\begin{aligned}
& T_{n}(f)=L T_{n}^{k}(1, f)+\left[L T_{n}^{k}\left(1, f_{m}\right)-L T_{n}^{k}(1, f)\right] \\
& +\left(R_{n, m}+R_{n, m, k}\right)+\left(N_{n, m}+N_{n, m, k}\right), \\
& \operatorname{rank}\left(R_{n, m}+R_{n, m, k}\right) \leq(c(m)+c(m, k)) n, \\
& \left\|N_{n, m}+N_{n, m, k}\right\| \leq \omega(m)+\omega(m, k), \\
& \left\|L T_{n}^{k}\left(1, f_{m}\right)-L T_{n}^{k}(1, f)\right\|_{1}=\left\|L T_{n}^{k}\left(1, f_{m}-f\right)\right\|_{1} \leq n\left\|f_{m}-f\right\|_{L^{1}},
\end{aligned}
$$

where the last inequality follows from (3.4). Let $\{m(k)\}_{k}$ be a sequence such that $m(k) \rightarrow \infty$ and

$$
\lim _{k \rightarrow \infty} c(m(k), k)=\lim _{k \rightarrow \infty} \omega(m(k), k)=0 .
$$

Note that such a sequence exists by Lemma 3.10 (apply the lemma with $x(m, k)=c(m, k)+$ $\omega(m, k))$. Then, for every $k$ and every $n \geq \max \left(n_{m(k)}, n_{m(k), k}\right)$,

$$
\begin{aligned}
& T_{n}(f)=L T_{n}^{k}(1, f)+\left[L T_{n}^{k}\left(1, f_{m(k)}\right)-L T_{n}^{k}(1, f)\right] \\
& +\left(R_{n, m(k)}+R_{n, m(k), k}\right)+\left(N_{n, m(k)}+N_{n, m(k), k}\right), \\
& \operatorname{rank}\left(R_{n, m(k)}+R_{n, m(k), k}\right) \leq(c(m(k))+c(m(k), k)) n, \\
& \left\|N_{n, m(k)}+N_{n, m(k), k}\right\| \leq \omega(m(k))+\omega(m(k), k), \\
& \left\|L T_{n}^{k}\left(1, f_{m(k)}\right)-L T_{n}^{k}(1, f)\right\|_{1} \leq n\left\|f_{m(k)}-f\right\|_{L^{1}} .
\end{aligned}
$$

The application of Lemma 3.9 allows one to decompose $L T_{n}^{k}\left(1, f_{m(k)}\right)-L T_{n}^{k}(1, f)$ as the sum of a small-rank term $\hat{R}_{n, k}$, with the rank bounded by $\sqrt{\left\|f_{m(k)}-f\right\|_{L^{1}}} n$, plus a small-norm term $\hat{N}_{n, k}$ with norm bounded by $\sqrt{\left\|f_{m(k)}-f\right\|_{L^{1}}}$. This shows that $\left\{L T_{n}^{k}(1, f)\right\}_{n} \stackrel{\text { a.c.s. }}{\longrightarrow}\left\{T_{n}(f)\right\}_{n}$, hence $\left\{T_{n}(f)\right\}_{n} \sim_{\text {LT }} f(\theta)$.

3.4. Singular value and spectral distribution of sums of products of block LT sequences. The main results of this section are Theorems 3.14 and 3.15. In order to prove them, we shall need the following lemma.

LEMMA 3.13. If $\left\{A_{n}\right\}_{n} \sim_{\mathrm{LT}} a(x) f(\theta)$, then $\left\{A_{n}\right\}_{n} \sim_{\sigma} a(x) f(\theta)$ and $\left\{A_{n}\right\}_{n}$ is s.u.

Proof. We have:

- $\left\{L T_{n}^{m}(a, f)\right\}_{n} \stackrel{\text { a.c.s. }}{\longrightarrow}\left\{A_{n}\right\}_{n}$ by definition of block LT sequences;

- $\left\{L T_{n}^{m}(a, f)\right\}_{n} \sim_{\sigma} a_{m}(x) f(\theta)$ with $a_{m}(x)=\sum_{k=1}^{m} a\left(\frac{k}{m}\right) \chi_{\left[\frac{k-1}{m}, \frac{k}{m}\right.}(x)$ by Proposition 3.6;

- $a_{m}(x) f(\theta) \rightarrow a(x) f(\theta)$ a.e. (and hence also in measure) by Lemma 2.7 because $a(x)$ is Riemann-integrable. 
We conclude that $\left\{A_{n}\right\}_{n} \sim_{\sigma} a(x) f(\theta)$ by Theorem 2.33, and so $\left\{A_{n}\right\}_{n}$ is s.u. by Proposition 2.20 .

THEOREM 3.14. If $\left\{A_{n}^{(i, j)}\right\}_{n} \sim_{\mathrm{LT}} a^{(i, j)}(x) f^{(i, j)}(\theta)$, for $i=1, \ldots, p$ and $j=1, \ldots, q_{i}$, then

$$
\left\{\sum_{i=1}^{p} \prod_{j=1}^{q_{i}} A_{n}^{(i, j)}\right\}_{n} \sim_{\sigma} \sum_{i=1}^{p} \prod_{j=1}^{q_{i}} a^{(i, j)}(x) f^{(i, j)}(\theta) .
$$

Proof. Let

$$
\begin{aligned}
A_{n} & =\sum_{i=1}^{p} \prod_{j=1}^{q_{i}} A_{n}^{(i, j)}, & A_{n, m} & =\sum_{i=1}^{p} \prod_{j=1}^{q_{i}} L T_{n}^{m}\left(a^{(i, j)}, f^{(i, j)}\right), \\
\kappa(x, \theta) & =\sum_{i=1}^{p} \prod_{j=1}^{q_{i}} a^{(i, j)}(x) f^{(i, j)}(\theta), & \kappa_{m}(x, \theta) & =\sum_{i=1}^{p} \prod_{j=1}^{q_{i}} a_{m}^{(i, j)}(x) f^{(i, j)}(\theta),
\end{aligned}
$$

where

$$
a_{m}^{(i, j)}(x)=\sum_{k=1}^{m} a^{(i, j)}\left(\frac{k}{m}\right) \chi_{\left[\frac{k-1}{m}, \frac{k}{m}\right)}(x) .
$$

Since $\left\{L T_{n}^{m}\left(a^{(i, j)}, f^{(i, j)}\right)\right\}_{n} \stackrel{\text { a.c.s. }}{\longrightarrow}\left\{A_{n}^{(i, j)}\right\}_{n}$ by definition of block LT sequences, we have:

- $\left\{A_{n, m}\right\}_{n} \stackrel{\text { a.c.s. }}{\longrightarrow}\left\{A_{n}\right\}_{n}$ by Lemma 3.13, Proposition 2.19, and Theorem 2.36;

- $\left\{A_{n, m}\right\}_{n} \sim_{\sigma} \kappa_{m}(x, \theta)$ by Theorem 3.4;

- $\kappa_{m}(x, \theta) \rightarrow \kappa(x, \theta)$ a.e. (and hence also in measure) by Lemma 2.7 because each $a^{(i, j)}(x)$ is Riemann-integrable.

We conclude that $\left\{A_{n}\right\}_{n} \sim_{\sigma} \kappa(x, \theta)$ by Theorem 2.33.

THEOREM 3.15. If $\left\{A_{n}^{(i, j)}\right\}_{n} \sim_{\mathrm{LT}} a^{(i, j)}(x) f^{(i, j)}(\theta)$, for $i=1, \ldots, p$ and $j=1, \ldots, q_{i}$, then

$$
\left\{\Re\left(\sum_{i=1}^{p} \prod_{j=1}^{q_{i}} A_{n}^{(i, j)}\right)\right\}_{n} \sim_{\lambda} \Re\left(\sum_{i=1}^{p} \prod_{j=1}^{q_{i}} a^{(i, j)}(x) f^{(i, j)}(\theta)\right) .
$$

Proof. The proof is essentially the same as the proof of Theorem 3.14. Define the matrices $A_{n}, A_{n, m}$ and the functions $\kappa(x, \theta), \kappa_{m}(x, \theta)$ as in the proof of Theorem 3.14. Since $\left\{L T_{n}^{m}\left(a^{(i, j)}, f^{(i, j)}\right)\right\}_{n} \stackrel{\text { a.c.s. }}{\longrightarrow}\left\{A_{n}^{(i, j)}\right\}_{n}$ by definition of block LT sequences, we have:

- $\left\{\Re\left(A_{n, m}\right)\right\}_{n} \stackrel{\text { a.c.s. }}{\longrightarrow}\left\{\Re\left(A_{n}\right)\right\}_{n}$ by Lemma 3.13, Proposition 2.19, and Theorem 2.36;

- $\left\{\Re\left(A_{n, m}\right)\right\}_{n} \sim_{\lambda} \Re\left(\kappa_{m}(x, \theta)\right)$ by Theorem 3.5;

- $\Re\left(\kappa_{m}(x, \theta)\right) \rightarrow \Re(\kappa(x, \theta))$ a.e. (and hence also in measure) by Lemma 2.7 because each $a^{(i, j)}(x)$ is Riemann-integrable.

We conclude that $\left\{\Re\left(A_{n}\right)\right\}_{n} \sim_{\lambda} \Re(\kappa(x, \theta))$ by Theorem 2.34.

3.5. Algebraic properties of block LT sequences. Proposition 3.16 collects the most elementary algebraic properties of block LT sequences, which follow from Definition 3.7, the properties of the block LT operator (see (3.1)-(3.3)), and Theorem 2.36.

PROPOSITION 3.16. The following properties hold.

- If $\left\{A_{n}\right\}_{n} \sim_{\mathrm{LT}} a(x) f(\theta)$, then $\left\{A_{n}^{*}\right\}_{n} \sim_{\mathrm{LT}} \overline{a(x)} f(\theta)^{*}=(a(x) f(\theta))^{*}$.

- If $\left\{A_{n}\right\}_{n} \sim_{\mathrm{LT}} a(x) f(\theta)$, then $\left\{\alpha A_{n}\right\}_{n} \sim_{\mathrm{LT}} \alpha a(x) f(\theta)$ for all $\alpha \in \mathbb{C}$.

- If $\left\{A_{n}^{(i)}\right\}_{n} \sim_{\mathrm{LT}} a(x) f_{i}(\theta), i=1, \ldots, r$, then $\left\{\sum_{i=1}^{r} A_{n}^{(i)}\right\}_{n} \sim_{\mathrm{LT}} a(x) \sum_{i=1}^{r} f_{i}(\theta)$. 
- If $\left\{A_{n}^{(i)}\right\}_{n} \sim_{\mathrm{LT}} a_{i}(x) f(\theta), i=1, \ldots, r$, then $\left\{\sum_{i=1}^{r} A_{n}^{(i)}\right\}_{n} \sim_{\mathrm{LT}} \sum_{i=1}^{r} a_{i}(x) f(\theta)$.

In Theorem 3.17, we show, under mild assumptions, that the product of block LT sequences is again a block LT sequence with symbol given by the product of the symbols.

THEOREM 3.17. Suppose that $\left\{A_{n}\right\}_{n} \sim_{\mathrm{LT}} a(x) f(\theta)$ and $\left\{\tilde{A}_{n}\right\}_{n} \sim_{\mathrm{LT}} \tilde{a}(x) \tilde{f}(\theta)$, where $f \in L^{p}([-\pi, \pi], s), \tilde{f} \in L^{q}([-\pi, \pi], s)$, and $1 \leq p, q \leq \infty$ are conjugate exponents. Then

$$
\left\{A_{n} \tilde{A}_{n}\right\}_{n} \sim_{\mathrm{LT}} a(x) \tilde{a}(x) f(\theta) \tilde{f}(\theta) .
$$

Proof. By Lemma 3.13, every block LT sequence is s.u., so in particular $\left\{A_{n}\right\}_{n}$ and $\left\{\tilde{A}_{n}\right\}_{n}$ are s.u. Since, by definition of block LT sequences,

$$
\left\{L T_{n}^{m}(a, f)\right\}_{n} \stackrel{\text { a.c.s. }}{\longrightarrow}\left\{A_{n}\right\}_{n}, \quad\left\{L T_{n}^{m}(\tilde{a}, \tilde{f})\right\}_{n} \stackrel{\text { a.c.s. }}{\longrightarrow}\left\{\tilde{A}_{n}\right\}_{n},
$$

Theorem 2.36 yields

$$
\left\{L T_{n}^{m}(a, f) L T_{n}^{m}(\tilde{a}, \tilde{f})\right\}_{n} \stackrel{\text { a.c.s. }}{\longrightarrow}\left\{A_{n} \tilde{A}_{n}\right\}_{n} .
$$

Using Proposition 3.3, especially (3.6), we obtain

$$
\left\{L T_{n}^{m}(a \tilde{a}, f \tilde{f})\right\}_{n} \stackrel{\text { a.c.s. }}{\longrightarrow}\left\{A_{n} \tilde{A}_{n}\right\}_{n},
$$

hence $\left\{A_{n} \tilde{A}_{n}\right\}_{n} \sim_{\text {LT }} a(x) \tilde{a}(x) f(\theta) \tilde{f}(\theta)$.

As a consequence of Theorems 3.11,3.12, and 3.17, we immediately obtain the following result.

THEOREM 3.18. If a $:[0,1] \rightarrow \mathbb{C}$ is Riemann-integrable and $f \in L^{1}([-\pi, \pi], s)$, then $\left\{D_{n, s}(a) T_{n}(f)\right\}_{n} \sim_{\mathrm{LT}} a(x) f(\theta)$.

3.6. Characterizations of block LT sequences. Theorem 3.18 shows that, for any $a, f$ as in Definition 3.7, there always exists an $s$-block matrix-sequence $\left\{A_{n}\right\}_{n}$ such that $\left\{A_{n}\right\}_{n} \sim_{\text {LT }} a(x) f(\theta)$. Indeed, it suffices to take $A_{n}=D_{n, s}(a) T_{n}(f)$. Theorem 3.19 shows that the sequences of the form $\left\{D_{n, s}(a) T_{n}(f)\right\}_{n}$ play a central role in the world of block LT sequences. Indeed,

$$
\left\{A_{n}\right\}_{n} \sim_{\mathrm{LT}} a(x) f(\theta) \Longleftrightarrow d_{\text {a.c.s. }}\left(\left\{A_{n}\right\}_{n},\left\{D_{n, s}(a) T_{n}(f)\right\}_{n}\right)=0 .
$$

THEOREM 3.19. Let $\left\{A_{n}\right\}_{n}$ be an s-block matrix-sequence, let $a:[0,1] \rightarrow \mathbb{C}$ be a Riemann-integrable function, and let $f \in L^{1}([-\pi, \pi], s)$. The following conditions are equivalent.

1. $\left\{A_{n}\right\}_{n} \sim_{\mathrm{LT}} a(x) f(\theta)$.

2. For all sequences $\left\{a_{m}\right\}_{m},\left\{f_{m}\right\}_{m},\left\{\left\{A_{n}^{(m)}\right\}_{n}\right\}_{m}$ such that

- $a_{m}:[0,1] \rightarrow \mathbb{C}$ is Riemann-integrable and $f_{m} \in L^{1}([-\pi, \pi], s)$,

- $a_{m}(x) f_{m}(\theta) \rightarrow a(x) f(\theta)$ in measure,

- $\left\{A_{n}^{(m)}\right\}_{n} \sim_{\mathrm{LT}} a_{m}(x) f_{m}(\theta)$,

we have $\left\{A_{n}^{(m)}\right\}_{n} \stackrel{\text { a.c.s. }}{\longrightarrow}\left\{A_{n}\right\}_{n}$.

3. There exist sequences $\left\{a_{m}\right\}_{m},\left\{f_{m}\right\}_{m}$ such that

- $a_{m}:[0,1] \rightarrow \mathbb{C}$ is continuous, $\left\|a_{m}\right\|_{\infty} \leq\|a\|_{L^{\infty}}$ for all $m$, and $a_{m} \rightarrow$ a a.e.,

- $f_{m}:[-\pi, \pi] \rightarrow \mathbb{C}^{s \times s}$ is a matrix-valued trigonometric polynomial, $\left\|\left(f_{m}\right)_{\alpha \beta}\right\|_{\infty} \leq$ ess $\sup _{[-\pi, \pi]}\left|f_{\alpha \beta}\right|$ for all $m$ and all $\alpha, \beta=1, \ldots, s$, and $f_{m} \rightarrow f$ a.e. and in $L^{1}([-\pi, \pi], s)$,

- $\left\{D_{n, s}\left(a_{m}\right) T_{n}\left(f_{m}\right)\right\}_{n} \stackrel{\text { a.c.s. }}{\longrightarrow}\left\{A_{n}\right\}_{n}$.

4. There exist sequences $\left\{a_{m}\right\}_{m},\left\{f_{m}\right\}_{m},\left\{\left\{A_{n}^{(m)}\right\}_{n}\right\}_{m}$ such that 
- $a_{m}:[0,1] \rightarrow \mathbb{C}$ is Riemann-integrable and $f_{m} \in L^{1}([-\pi, \pi], s)$,

- $a_{m}(x) f_{m}(\theta) \rightarrow a(x) f(\theta)$ in measure,

- $\left\{A_{n}^{(m)}\right\}_{n} \sim_{\mathrm{LT}} a_{m}(x) f_{m}(\theta)$ and $\left\{A_{n}^{(m)}\right\}_{n} \stackrel{\text { a.c.s. }}{\longrightarrow}\left\{A_{n}\right\}_{n}$.

5. $\left\{D_{n, s}(a) T_{n}(f)\right\}_{n} \stackrel{\text { a.c.s. }}{\longrightarrow}\left\{A_{n}\right\}_{n}$.

6. $A_{n}=D_{n, s}(a) T_{n}(f)+Z_{n}$, for every $n$, where $\left\{Z_{n}\right\}_{n}$ is zero-distributed.

Proof. $(1 \Longrightarrow 2)$ Suppose that $\left\{A_{n}\right\}_{n} \sim_{\mathrm{LT}} a(x) f(\theta)$, and let $\left\{a_{m}\right\}_{m},\left\{f_{m}\right\}_{m}$, $\left\{\left\{A_{n}^{(m)}\right\}_{n}\right\}_{m}$ be sequences with the properties specified in item 2. By Theorems 2.32 and 3.14, we have

$$
\begin{aligned}
d_{\text {a.c.s. }}\left(\left\{A_{n}^{(m)}\right\}_{n},\left\{A_{n}\right\}_{n}\right) & =p_{\text {a.c.s. }}\left(\left\{A_{n}^{(m)}-A_{n}\right\}_{n}\right) \\
& =p_{\text {measure }}\left(a_{m}(x) f_{m}(\theta)-a(x) f(\theta)\right) \\
& =d_{\text {measure }}\left(a_{m}(x) f_{m}(\theta), a(x) f(\theta)\right),
\end{aligned}
$$

which tends to 0 as $m \rightarrow \infty$ because $a_{m}(x) f_{m}(\theta) \rightarrow a(x) f(\theta)$ in measure.

$(2 \Longrightarrow 3)$ Since any Riemann-integrable function is bounded by definition, we have $a \in L^{\infty}([0,1])$. Hence, by [50, Theorem 2.2], there exists a sequence of continuous functions $a_{m}:[0,1] \rightarrow \mathbb{C}$ such that $\left\|a_{m}\right\|_{\infty} \leq\|a\|_{L^{\infty}}$ for all $m$ and $a_{m} \rightarrow a$ a.e. The sequence $\left\{a_{m}\right\}_{m}$ satisfies the properties in item 3 . Note also that $a_{m} \rightarrow a$ in $L^{1}([0,1])$ by the dominated convergence theorem.

Since $f \in L^{1}([-\pi, \pi], s)$, by [50, Lemma 2.7], there exists a sequence of matrix-valued trigonometric polynomials $f_{m}:[-\pi, \pi] \rightarrow \mathbb{C}^{s \times s}$ such that $\left\|\left(f_{m}\right)_{\alpha \beta}\right\|_{\infty} \leq \operatorname{ess} \sup _{[-\pi, \pi]}\left|f_{\alpha \beta}\right|$ for all $m$ and all $\alpha, \beta=1, \ldots, s$, and $f_{m} \rightarrow f$ a.e. and in $L^{1}([-\pi, \pi], s)$. The sequence $\left\{f_{m}\right\}_{m}$ satisfies the properties in item 3 .

Since $a_{m}(x) f_{m}(\theta) \rightarrow a(x) f(\theta)$ in $L^{1}([0,1] \times[-\pi, \pi], s)$ (and hence also in measure), item 2 and Theorem 3.18 imply that $\left\{D_{n, s}\left(a_{m}\right) T_{n}\left(f_{m}\right)\right\}_{n} \stackrel{\text { a.c.s. }}{\longrightarrow}\left\{A_{n}\right\}_{n}$, and the proof is complete.

(3 $\Longrightarrow 4$ ) Simply note that, under the assumptions in item $3, a_{m}(x) f_{m}(\theta) \rightarrow a(x) f(\theta)$ in measure, and $\left\{D_{n, s}\left(a_{m}\right) T_{n}\left(f_{m}\right)\right\}_{n} \sim_{\mathrm{LT}} a_{m}(x) f_{m}(\theta)$ by Theorem 3.18.

$(4 \Longrightarrow 1)$ Let $\left\{B_{n}\right\}_{n} \sim_{\mathrm{LT}} a(x) f(\theta)$; we can take, e.g., $B_{n}=D_{n, s}(a) T_{n}(f)$ thanks to Theorem 3.18. By Theorems 2.32 and 3.14,

$$
\begin{aligned}
d_{\text {a.c.s. }}\left(\left\{A_{n}^{(m)}\right\}_{n},\left\{B_{n}\right\}_{n}\right) & =p_{\text {a.c.s. }}\left(\left\{A_{n}^{(m)}-B_{n}\right\}_{n}\right) \\
& =p_{\text {measure }}\left(a_{m}(x) f_{m}(\theta)-a(x) f(\theta)\right) \\
& =d_{\text {measure }}\left(a_{m}(x) f_{m}(\theta), a(x) f(\theta)\right),
\end{aligned}
$$

which tends to 0 as $m \rightarrow \infty$ because $a_{m}(x) f_{m}(\theta) \rightarrow a(x) f(\theta)$ in measure. As a consequence, $\left\{A_{n}^{(m)}\right\}_{n} \stackrel{\text { a.c.s. }}{\longrightarrow}\left\{B_{n}\right\}_{n}$, and since $\left\{A_{n}^{(m)}\right\}_{n} \stackrel{\text { a.c.s. }}{\longrightarrow}\left\{A_{n}\right\}_{n}$ by assumption, we conclude that $d_{\text {a.c.s. }}\left(\left\{A_{n}\right\}_{n},\left\{B_{n}\right\}_{n}\right)=0$. Considering that $\left\{L T_{n}^{m}(a, f)\right\}_{n} \stackrel{\text { a.c.s. }}{\longrightarrow}\left\{B_{n}\right\}_{n}$, we infer that $\left\{L T_{n}^{m}(a, f)\right\}_{n} \stackrel{\text { a.c.s. }}{\longrightarrow}\left\{A_{n}\right\}_{n}$, i.e., $\left\{A_{n}\right\}_{n} \sim_{\text {LT }} a(x) f(\theta)$.

(5 $\Longleftrightarrow 6$ ) Item 5 is equivalent to $d_{\text {a.c.s. }}\left(\left\{A_{n}\right\}_{n},\left\{D_{n, s}(a) T_{n}(f)\right\}_{n}\right)=0$, which, by Theorem 2.30, is equivalent to $\left\{A_{n}-D_{n, s}(a) T_{n}(f)\right\}_{n} \sim_{\sigma} 0$.

$\left(2 \Longrightarrow 5\right.$ ) Obvious (take $a_{m}=a, f_{m}=f$ and $A_{n}^{(m)}=D_{n, s}(a) T_{n}(f)$ ).

$\left(5 \Longrightarrow 4\right.$ ) Obvious (take $a_{m}=a, f_{m}=f$ and $A_{n}^{(m)}=D_{n, s}(a) T_{n}(f)$ ).

4. Block generalized locally Toeplitz sequences. In this chapter we develop the theory of block GLT sequences by correcting and extending the results in [76, Section 3.3] and [56]. 
4.1. Equivalent definitions of block GLT sequences. Block GLT sequences can be defined in several different ways. We begin with what we may call the "classical definition".

DEFINITION 4.1 (Block generalized locally Toeplitz sequences). Let $\left\{A_{n}\right\}_{n}$ be an sblock matrix-sequence, and let $\kappa:[0,1] \times[-\pi, \pi] \rightarrow \mathbb{C}^{s \times s}$ be measurable. We say that $\left\{A_{n}\right\}_{n}$ is an (s-block) generalized locally Toeplitz $(G L T)$ sequence with symbol $\kappa$, and we write $\left\{A_{n}\right\}_{n} \sim_{\mathrm{GLT}} \kappa$, if the following condition is met.

For every $m \in \mathbb{N}$ there exists a finite number of s-block LT sequences $\left\{A_{n, m}^{(i, j)}\right\}_{n} \sim_{\mathrm{LT}} a_{m}^{(i, j)}(x) f_{m}^{(i, j)}(\theta), i=1, \ldots, N_{m}, j=1, \ldots, M_{m, i}$, such that:

- $\sum_{i=1}^{N_{m}} \prod_{j=1}^{M_{m, i}} a_{m}^{(i, j)}(x) f_{m}^{(i, j)}(\theta) \rightarrow \kappa(x, \theta)$ in measure;

- $\left\{\sum_{i=1}^{N_{m}} \prod_{j=1}^{M_{m, i}} A_{n, m}^{(i, j)}\right\}_{n} \stackrel{\text { a.c.s. }}{\longrightarrow}\left\{A_{n}\right\}_{n}$.

In what follows, unless specified otherwise, whenever we write a relation such as $\left\{A_{n}\right\}_{n} \sim_{\mathrm{GLT}} \kappa$, it is understood that $\left\{A_{n}\right\}_{n}$ is an $s$-block matrix-sequence and $\kappa:[0,1] \times$ $[-\pi, \pi] \rightarrow \mathbb{C}^{s \times s}$ is measurable, as in Definition 4.1. In the case $s=1$, it can be shown that Definition 4.1 is equivalent to the definition of (scalar) GLT sequences given in [50, Chapter 8], that is, the set of GLT sequences defined here for $s=1$ is the same as the set of GLT sequences defined in [50, Chapter 8].

REMARK 4.2. It is clear that any sum of products of $s$-block LT sequences is an $s$-block GLT sequence. More precisely, if $\left\{A_{n}^{(i, j)}\right\}_{n} \sim_{\mathrm{LT}} a^{(i, j)}(x) f^{(i, j)}(\theta)$, for $i=1, \ldots, p$ and $j=1, \ldots, q_{i}$, then

$$
\left\{\sum_{i=1}^{p} \prod_{j=1}^{q_{i}} A_{n}^{(i, j)}\right\}_{n} \sim_{\mathrm{GLT}} \sum_{i=1}^{p} \prod_{j=1}^{q_{i}} a^{(i, j)}(x) f^{(i, j)}(\theta) .
$$

REMARK 4.3. If $a:[0,1] \rightarrow \mathbb{C}^{s \times s}$, then

$$
D_{n}(a)=\sum_{i, j=1}^{s} D_{n}\left(a_{i j} E_{i j}^{(s)}\right)=\sum_{i, j=1}^{s} D_{n}\left(a_{i j} I_{s}\right) D_{n}\left(E_{i j}^{(s)}\right)=\sum_{i, j=1}^{s} D_{n, s}\left(a_{i j}\right) T_{n}\left(E_{i j}^{(s)}\right) .
$$

Hence, by Remark 4.2 and Theorem 3.18, if $a$ is Riemann-integrable, then

$$
\left\{D_{n}(a)\right\}_{n} \sim_{\mathrm{GLT}} \sum_{i, j=1}^{s} a_{i j}(x) E_{i j}^{(s)}=a(x) .
$$

REMARK 4.4. Let $\left\{A_{n}\right\}_{n} \sim_{\mathrm{GLT}} \kappa$ and $\left\{B_{n}\right\}_{n} \sim_{\mathrm{GLT}} \xi$. Then, $\left\{A_{n}^{*}\right\}_{n} \sim_{\mathrm{GLT}} \kappa^{*}$ and $\left\{\alpha A_{n}+\beta B_{n}\right\}_{n} \sim_{\text {GLT }} \alpha \kappa+\beta \xi$ for all $\alpha, \beta \in \mathbb{C}$. This follows immediately from Definition 4.1, Proposition 3.16, and Theorem 2.36.

In the remainder of this section, we present another equivalent definition of block GLT sequences, which is illuminating for many purposes. Let

$$
\begin{aligned}
\mathscr{E}^{(s)} & =\left\{\left\{A_{n}\right\}_{n}:\left\{A_{n}\right\}_{n} \text { is an } s \text {-block matrix-sequence }\right\}, \\
\mathfrak{M}^{(s)} & =\left\{\kappa:[0,1] \times[-\pi, \pi] \rightarrow \mathbb{C}^{s \times s}: \kappa \text { is measurable }\right\}, \\
\mathscr{E}^{(s)} \times \mathfrak{M}^{(s)} & =\left\{\left(\left\{A_{n}\right\}_{n}, \kappa\right):\left\{A_{n}\right\}_{n} \in \mathscr{E}^{(s)}, \kappa \in \mathfrak{M}^{(s)}\right\} .
\end{aligned}
$$

We make the following observations.

- $\mathscr{E}^{(s)}$ is a *-algebra with respect to the natural pointwise operations (namely, $\left.\left\{A_{n}\right\}_{n}^{*}=\left\{A_{n}^{*}\right\}_{n}, \alpha\left\{A_{n}\right\}_{n}+\beta\left\{B_{n}\right\}_{n}=\left\{\alpha A_{n}+\beta B_{n}\right\}_{n},\left\{A_{n}\right\}_{n}\left\{B_{n}\right\}_{n}=\left\{A_{n} B_{n}\right\}_{n}\right)$, and it is also a pseudometric space with respect to the pseudometric $d_{\text {a.c.s. }}$ inducing the a.c.s. topology $\tau_{\text {a.c.s. }}$. 
- $\mathfrak{M}^{(s)}$ is a *-algebra with respect to the natural pointwise operations, and it is also a pseudometric space with respect to the pseudometric $d_{\text {measure }}$ inducing the topology $\tau_{\text {measure }}$ of convergence in measure.

- $\mathscr{E}^{(s)} \times \mathfrak{M}^{(s)}$ is a *-algebra with respect to the natural pointwise operations (namely, $\left(\left\{A_{n}\right\}_{n}, \kappa\right)^{*}=\left(\left\{A_{n}^{*}\right\}_{n}, \kappa^{*}\right), \alpha\left(\left\{A_{n}\right\}_{n}, \kappa\right)+\beta\left(\left\{B_{n}\right\}_{n}, \xi\right)=\left(\left\{\alpha A_{n}+\beta B_{n}\right\}_{n}, \alpha \kappa+\beta \xi\right)$, $\left.\left(\left\{A_{n}\right\}_{n}, \kappa\right)\left(\left\{B_{n}\right\}_{n}, \xi\right)=\left(\left\{A_{n} B_{n}\right\}_{n}, \kappa \xi\right)\right)$, and it is also a pseudometric space with respect to the product pseudometric

$$
\left(d_{\text {a.c.s. }} \times d_{\text {measure }}\right)\left(\left(\left\{A_{n}\right\}_{n}, \kappa\right),\left(\left\{B_{n}\right\}_{n}, \xi\right)\right)=d_{\text {a.c.s. }}\left(\left\{A_{n}\right\}_{n},\left\{B_{n}\right\}_{n}\right)+d_{\text {measure }}(\kappa, \xi)
$$

inducing the product topology $\tau_{\text {a.c.s. }} \times \tau_{\text {measure }}$.

Let $\mathcal{A}^{(s)}$ be the *-subalgebra of $\mathscr{E}^{(s)} \times \mathfrak{M}^{(s)}$ generated by the set of " $s$-block LT pairs"

$$
\mathcal{L}^{(s)}=\left\{\left(\left\{A_{n}\right\}_{n}, a(x) f(\theta)\right) \in \mathscr{E}^{(s)} \times \mathfrak{M}^{(s)}:\left\{A_{n}\right\}_{n} \sim_{\mathrm{LT}} a(x) f(\theta)\right\} .
$$

Using Proposition 3.16, it is not difficult to see that

$$
\begin{aligned}
\mathcal{A}^{(s)}=\{ & \left(\sum_{i=1}^{p} \prod_{j=1}^{q_{i}} A_{n}^{(i, j)}, \sum_{i=1}^{p} \prod_{j=1}^{q_{i}} a^{(i, j)}(x) f^{(i, j)}(\theta)\right): \\
& \left.p, q_{1}, \ldots, q_{p} \in \mathbb{N}, \quad\left\{A_{n}^{(i, j)}\right\}_{n} \sim_{\mathrm{LT}} a^{(i, j)}(x) f^{(i, j)}(\theta) \text { for all } i, j\right\} .
\end{aligned}
$$

We can now reformulate Definition 4.1 as follows.

DEFINITION 4.5 (Block generalized locally Toeplitz sequence). Let $\left\{A_{n}\right\}_{n}$ be an s-block matrix-sequence, and let $\kappa:[0,1] \times[-\pi, \pi] \rightarrow \mathbb{C}^{s \times s}$ be measurable. We say that $\left\{A_{n}\right\}_{n}$ is an (s-block) generalized locally Toeplitz (GLT) sequence with symbol $\kappa$, and we write $\left\{A_{n}\right\}_{n} \sim_{\mathrm{GLT}} \kappa$, if the pair $\left(\left\{A_{n}\right\}_{n}, \kappa\right)$ belongs to the closure of $\mathcal{A}^{(s)}$ in $\left(\mathscr{E}(s) \times \mathfrak{M}^{(s)}, d_{\text {a.c.s. }} \times d_{\text {measure }}\right)$. In other words, the set of "s-block GLT pairs"

$$
\mathcal{G}^{(s)}=\left\{\left(\left\{A_{n}\right\}_{n}, \kappa\right) \in \mathscr{E}^{(s)} \times \mathfrak{M}^{(s)}:\left\{A_{n}\right\}_{n} \sim_{\mathrm{GLT}} \kappa\right\} .
$$

is defined as the closure of $\mathcal{A}^{(s)}$ in $\left(\mathscr{E}^{(s)} \times \mathfrak{M}^{(s)}, d_{\text {a.c.s. }} \times d_{\text {measure }}\right)$.

In the light of this algebraic-topological definition of block GLT sequences, the following theorem is obvious.

THEOREM 4.6. Let $\left\{A_{n}\right\}_{n}$ be an s-block matrix-sequence, and let the function $\kappa:[0,1] \times[-\pi, \pi] \rightarrow \mathbb{C}^{s \times s}$ be measurable. Suppose that:

1. $\left\{B_{n, m}\right\}_{n} \sim_{\mathrm{GLT}} \kappa_{m}$ for every $m$;

2. $\left\{B_{n, m}\right\}_{n} \stackrel{\text { a.c.s. }}{\longrightarrow}\left\{A_{n}\right\}_{n}$;

3. $\kappa_{m} \rightarrow \kappa$ in measure.

Then $\left\{A_{n}\right\}_{n} \sim_{\mathrm{GLT}} \kappa$.

4.2. Singular value and spectral distribution of block GLT sequences. In this section we prove the main singular value and eigenvalue distribution results for block GLT sequences.

THEOREM 4.7. If $\left\{A_{n}\right\}_{n} \sim_{\mathrm{GLT}} \kappa$, then $\left\{A_{n}\right\}_{n} \sim_{\sigma} \kappa$.

Proof. By definition, for every $m \in \mathbb{N}$, there exist block LT sequences $\left\{A_{n, m}^{(i, j)}\right\}_{n} \sim_{\mathrm{LT}}$ $a_{m}^{(i, j)}(x) f_{m}^{(i, j)}(\theta), i=1, \ldots, N_{m}, j=1, \ldots, M_{m, i}$, such that:

- $\sum_{i=1}^{N_{m}} \prod_{j=1}^{M_{m, i}} a_{m}^{(i, j)}(x) f_{m}^{(i, j)}(\theta) \rightarrow \kappa(x, \theta)$ in measure;

- $\left\{\sum_{i=1}^{N_{m}} \prod_{j=1}^{M_{m, i}} A_{n, m}^{(i, j)}\right\}_{n} \stackrel{\text { a.c.s. }}{\longrightarrow}\left\{A_{n}\right\}_{n}$.

Moreover, by Theorem 3.14, 
- $\left\{\sum_{i=1}^{N_{m}} \prod_{j=1}^{M_{m, i}} A_{n, m}^{(i, j)}\right\}_{n} \sim_{\sigma} \sum_{i=1}^{N_{m}} \prod_{j=1}^{M_{m, i}} a_{m}^{(i, j)}(x) f_{m}^{(i, j)}(\theta)$.

We conclude that $\left\{A_{n}\right\}_{n} \sim_{\sigma} \kappa$ by Theorem 2.33.

REMARK 4.8. Any block GLT sequence $\left\{A_{n}\right\}_{n}$ is s.u. This follows from Theorem 4.7 and Proposition 2.20.

Using Theorem 4.7 we now show that the symbol of a block GLT sequence is essentially unique and that the symbol of a block GLT sequence formed by Hermitian matrices is Hermitian a.e.

PROPOSITION 4.9. If $\left\{A_{n}\right\}_{n} \sim_{\mathrm{GLT}} \kappa$ and $\left\{A_{n}\right\}_{n} \sim_{\mathrm{GLT}} \xi$, then $\kappa=\xi$ a.e.

Proof. By Remark 4.4 we have $\left\{O_{s n}\right\}_{n}=\left\{A_{n}-A_{n}\right\}_{n} \sim_{\mathrm{GLT}} \kappa-\xi$. Hence, by Theorem 4.7, we also have $\left\{O_{s n}\right\}_{n} \sim_{\sigma} \kappa-\xi$, i.e.,

$$
F(0)=\frac{1}{2 \pi} \int_{0}^{1} \int_{-\pi}^{\pi} \frac{\sum_{j=1}^{s} F\left(\sigma_{j}(\kappa(x, \theta)-\xi(x, \theta))\right)}{s} \mathrm{~d} \theta \mathrm{d} x, \quad \forall F \in C_{c}(\mathbb{R}) .
$$

We conclude that $\kappa-\xi=O_{s}$ a.e. by Remark 2.6.

PROPOSITION 4.10. If $\left\{A_{n}\right\}_{n} \sim_{\text {GLT }} \kappa$ and the $A_{n}$ are Hermitian, then $\kappa$ is Hermitian a.e.

Proof. Since the $A_{n}$ are Hermitian, by Remark 4.4, we have $\left\{A_{n}\right\}_{n} \sim_{\text {GLT }} \kappa$ and $\left\{A_{n}\right\}_{n} \sim_{\mathrm{GLT}} \kappa^{*}$. Thus, by Proposition $4.9, \kappa=\kappa^{*}$ a.e.

THEOREM 4.11. If $\left\{A_{n}\right\}_{n} \sim_{\mathrm{GLT}} \kappa$ and the $A_{n}$ are Hermitian, then $\left\{A_{n}\right\}_{n} \sim_{\lambda} \kappa$.

Proof. By definition, for every $m \in \mathbb{N}$ there exist block LT sequences $\left\{A_{n, m}^{(i, j)}\right\}_{n} \sim_{\mathrm{LT}}$ $a_{m}^{(i, j)}(x) f_{m}^{(i, j)}(\theta), i=1, \ldots, N_{m}, j=1, \ldots, M_{m, i}$, such that:

- $\sum_{i=1}^{N_{m}} \prod_{j=1}^{M_{m, i}} a_{m}^{(i, j)}(x) f_{m}^{(i, j)}(\theta) \rightarrow \kappa(x, \theta)$ in measure;

- $\left\{\sum_{i=1}^{N_{m}} \prod_{j=1}^{M_{m, i}} A_{n, m}^{(i, j)}\right\}_{n} \stackrel{\text { a.c.s. }}{\longrightarrow}\left\{A_{n}\right\}_{n}$.

Thus:

- $\left\{\Re\left(\sum_{i=1}^{N_{m}} \prod_{j=1}^{M_{m, i}} A_{n, m}^{(i, j)}\right)\right\}_{n} \stackrel{\text { a.c.s. }}{\longrightarrow}\left\{\Re\left(A_{n}\right)\right\}_{n}$ by Theorem 2.36;

- $\left\{\Re\left(\sum_{i=1}^{N_{m}} \prod_{j=1}^{M_{m, i}} A_{n, m}^{(i, j)}\right)\right\}_{n} \sim_{\lambda} \Re\left(\sum_{i=1}^{N_{m}} \prod_{j=1}^{M_{m, i}} a_{m}^{(i, j)}(x) f_{m}^{(i, j)}(\theta)\right)$ by Theorem 3.15;

- $\Re\left(\sum_{i=1}^{N_{m}} \prod_{j=1}^{M_{m, i}} a_{m}^{(i, j)}(x) f_{m}^{(i, j)}(\theta)\right) \rightarrow \Re(\kappa(x, \theta))$ in measure.

We conclude that $\left\{\Re\left(A_{n}\right)\right\}_{n} \sim_{\lambda} \Re(\kappa)$ by Theorem 2.34. Since the matrices $A_{n}$ are Hermitian, we have $\Re\left(A_{n}\right)=A_{n}$ and $\Re(\kappa)=\kappa$ a.e. by Proposition 4.10. Hence, the spectral distribution $\left\{\Re\left(A_{n}\right)\right\}_{n} \sim_{\lambda} \Re(\kappa)$ yields $\left\{A_{n}\right\}_{n} \sim_{\lambda} \kappa$.

We end this section with a spectral distribution result for (compressions of) block GLT sequences formed by perturbed Hermitian matrices.

THEOREM 4.12. Let $\left\{A_{n}\right\}_{n} \sim_{\mathrm{GLT}} \kappa$ and $A_{n}=X_{n}+Y_{n}$. Assume that

1. every $X_{n}$ is Hermitian,

2. $\left\|Y_{n}\right\|_{2}=o(\sqrt{n})$.

Then $\left\{P_{n}^{*} A_{n} P_{n}\right\}_{n} \sim_{\sigma, \lambda} \kappa$ for every sequence $\left\{P_{n}\right\}_{n}$ such that $P_{n} \in \mathbb{C}^{s n \times \delta_{n}}, P_{n}^{*} P_{n}=I_{\delta_{n}}$, $\delta_{n} \leq$ sn, and $\delta_{n} / s n \rightarrow 1$. In particular, $\left\{A_{n}\right\}_{n} \sim_{\sigma, \lambda} \kappa\left(\right.$ take $\left.P_{n}=I_{s n}\right)$.

Proof. $\left\{Y_{n}\right\}_{n}$ is zero-distributed by Theorem 2.16, so $\left\{Y_{n}\right\}_{n} \sim_{\mathrm{GLT}} O_{s}$ by Theorem 3.8. Since $X_{n}=A_{n}-Y_{n}$ and the matrices $X_{n}$ are Hermitian, we have $\left\{X_{n}\right\}_{n} \sim_{\text {GLT }} \kappa$ by Remark 4.4 and $\left\{X_{n}\right\}_{n} \sim_{\sigma, \lambda} \kappa$ by Theorems 4.7 and 4.11. Consider the decomposition

$$
P_{n}^{*} A_{n} P_{n}=P_{n}^{*} X_{n} P_{n}+P_{n}^{*} Y_{n} P_{n}
$$

Let $\hat{P}_{n}=\left[P_{n} \mid O\right]$ be the $s n \times s n$ matrix obtained from $P_{n}$ by adding zeros, and observe that $\hat{P}_{n}^{*} B \hat{P}_{n}=P_{n}^{*} B P_{n} \oplus O_{s n-\delta_{n}}$ for all $B \in \mathbb{C}^{s n \times s n}$. We have:

- $\left\{P_{n}^{*} X_{n} P_{n}\right\}_{n} \sim_{\sigma, \lambda} \kappa$ by Theorem 2.28; 
- $\left\|P_{n}^{*} Y_{n} P_{n}\right\|_{2}=\left\|\hat{P}_{n}^{*} Y_{n} \hat{P}_{n}\right\|_{2} \leq\left\|\hat{P}_{n}^{*}\right\|\left\|Y_{n}\right\|_{2}\left\|\hat{P}_{n}\right\|=\left\|Y_{n}\right\|_{2}=o(\sqrt{n})=o\left(\sqrt{\delta_{n}}\right)$, where the inequality follows from [13, Proposition IV.2.4] and the unitary invariance of $\|\cdot\|_{2}$, while the second equality follows from the fact that the nonzero singular values of $\hat{P}_{n}$ and $\hat{P}_{n}^{*}$ are all equal to 1 due to the relation $\hat{P}_{n}^{*} \hat{P}_{n}=P_{n}^{*} P_{n} \oplus O_{s n-\delta_{n}}=I_{\delta_{n}} \oplus O_{s n-\delta_{n}}$; in particular, $\left\{P_{n}^{*} Y_{n} P_{n}\right\}_{n}$ is zero-distributed by Theorem 2.16.

We conclude that $d_{\text {a.c.s. }}\left(\left\{P_{n}^{*} X_{n} P_{n}\right\}_{n},\left\{P_{n}^{*} A_{n} P_{n}\right\}_{n}\right)=0$. The singular value distribution $\left\{P_{n}^{*} A_{n} P_{n}\right\}_{n} \sim_{\sigma} \kappa$ follows from Remark 2.35, and the spectral distribution $\left\{P_{n}^{*} A_{n} P_{n}\right\}_{n} \sim_{\lambda} \kappa$ follows from Theorem 2.26.

REMARK 4.13 (Extensions of the spectral distribution results to the non-Hermitian case). As shown in this section, the theory of block GLT sequences allows one to compute the spectral distribution of a given block GLT sequence $\left\{A_{n}\right\}_{n}$ in the case where $A_{n}$ is either a Hermitian matrix or a (small) perturbation of a Hermitian matrix. Similar (but less powerful) results can be formulated in the case where $A_{n}$ is either a normal matrix or a (small) perturbation of a normal matrix. The real challenge consists in obtaining spectral distribution results in the case where $A_{n}$ is a purely non-normal matrix. In this direction, the most promising tools can be found in [22, 81]. In [38, 39, 41], the tools from [81] have been refined and successfully employed in the case where $A_{n}$ belongs to the algebra generated by block Toeplitz matrices and their inverses. The main results obtained so far are [39, Theorems 9 and 10]; extending them further is certainly an interesting topic for future research.

4.3. Block GLT sequences and matrix-valued measurable functions. We prove in this section that every $s \times s$ matrix-valued measurable function $\kappa:[0,1] \times[-\pi, \pi] \rightarrow \mathbb{C}^{s \times s}$ is the symbol of a suitable $s$-block GLT sequence $\left\{A_{n}\right\}_{n}$. In combination with the results already proved before, this will allow us to show that the map associating with each $s$-block GLT sequence $\left\{A_{n}\right\}_{n}$ its symbol $\kappa$ is an isometry with respect to $d_{\text {a.c.s. }}$ on the space $\mathscr{G}^{(s)}$ of $s$-block GLT sequences and $d_{\text {measure }}$ on the space $\mathfrak{M}^{(s)}$ of $s \times s$ matrix-valued measurable functions defined on $[0,1] \times[-\pi, \pi]$.

LEMMA 4.14. Let $\kappa:[0,1] \times[-\pi, \pi] \rightarrow \mathbb{C}^{s \times s}$ be any measurable function. Then, there exists a sequence of s-block GLT pairs $\left(\left\{A_{n, m}\right\}_{n}, \kappa_{m}\right)$ such that $\kappa_{m} \rightarrow \kappa$ in measure.

Proof. By [50, Lemma 2.8], for every $\alpha, \beta=1, \ldots, s$, there exists a sequence of measurable functions $\kappa_{m, \alpha \beta}:[0,1] \times[-\pi, \pi] \rightarrow \mathbb{C}$ such that $\kappa_{m, \alpha \beta}$ is of the form

$$
\kappa_{m, \alpha \beta}(x, \theta)=\sum_{j=-N_{m}}^{N_{m}} a_{j, m, \alpha \beta}(x) e^{\mathrm{i} j \theta},
$$

with $N_{m} \in \mathbb{N}$ and $a_{j, m, \alpha \beta}:[0,1] \rightarrow \mathbb{C}$ belonging to $C^{\infty}([0,1])$, and $\kappa_{m, \alpha \beta} \rightarrow \kappa_{\alpha \beta}$ a.e. Take

$$
\begin{aligned}
\kappa_{m}(x, \theta) & =\left[\kappa_{m, \alpha \beta}(x, \theta)\right]_{\alpha, \beta=1}^{s} \\
& =\sum_{\alpha, \beta=1}^{s} \kappa_{m, \alpha \beta}(x, \theta) E_{\alpha \beta}^{(s)}=\sum_{\alpha, \beta=1}^{s} \sum_{j=-N_{m}}^{N_{m}} a_{j, m, \alpha \beta}(x) e^{\mathrm{i} j \theta} E_{\alpha \beta}^{(s)}, \\
A_{n, m} & =\sum_{\alpha, \beta=1}^{s} \sum_{j=-N_{m}}^{N_{m}} D_{n, s}\left(a_{j, m, \alpha \beta}\right) T_{n}\left(e^{\mathrm{i} j \theta} E_{\alpha \beta}^{(s)}\right) .
\end{aligned}
$$

Clearly, $\kappa_{m} \rightarrow \kappa$ a.e. and hence also in measure. Moreover, $\left\{A_{n, m}\right\}_{n} \sim_{\text {GLT }} \kappa_{m}$ by Theorem 3.18 and Remark 4.4.

THEOREM 4.15. Let $\kappa:[0,1] \times[-\pi, \pi] \rightarrow \mathbb{C}^{s \times s}$ be any measurable function. Then there exists an s-block matrix-sequence $\left\{A_{n}\right\}_{n}$ such that $\left\{A_{n}\right\}_{n} \sim_{\mathrm{GLT}} \kappa$.

Proof. By Lemma 4.14, there exist $s$-block GLT sequences $\left\{A_{n, m}\right\}_{n} \sim_{\text {GLT }} \kappa_{m}$ such that $\kappa_{m} \rightarrow \kappa$ in measure. Since $\left\{\kappa_{m}\right\}_{m}$ converges in measure, it is a Cauchy sequence with respect 
to the pseudometric $d_{\text {measure }}$ inducing the convergence in measure. It follows that $\left\{\left\{A_{n, m}\right\}_{n}\right\}_{m}$ is a Cauchy sequence with respect to the pseudometric $d_{\text {a.c.s. }}$ inducing the a.c.s. convergence because, for each pair of indices $m, m^{\prime}$, we have $\left\{A_{n, m}-A_{n, m^{\prime}}\right\}_{n} \sim_{\mathrm{GLT}} \kappa_{m}-\kappa_{m^{\prime}}$ by Remark 4.4, $\left\{A_{n, m}-A_{n, m^{\prime}}\right\}_{n} \sim_{\sigma} \kappa_{m}-\kappa_{m^{\prime}}$ by Theorem 4.7, and

$$
\begin{aligned}
d_{\text {a.c.s. }}\left(\left\{A_{n, m}\right\}_{n},\left\{A_{n, m^{\prime}}\right\}_{n}\right) & =p_{\text {a.c.s. }}\left(\left\{A_{n, m}-A_{n, m^{\prime}}\right\}_{n}\right) \\
& =p_{\text {measure }}\left(\kappa_{m}-\kappa_{m^{\prime}}\right) \\
& =d_{\text {measure }}\left(\kappa_{m}, \kappa_{m^{\prime}}\right)
\end{aligned}
$$

by Theorem 2.32. Since $d_{\text {a.c.s. }}$ is complete on the space $\mathscr{E}^{(s)}$ of $s$-block matrix-sequences (by Theorem 2.30), we infer that $\left\{A_{n, m}\right\}_{n} \stackrel{\text { a.c.s. }}{\longrightarrow}\left\{A_{n}\right\}_{n}$ for some $s$-block matrix-sequence $\left\{A_{n}\right\}_{n}$. We conclude that $\left\{A_{n}\right\}_{n} \sim_{\text {GLT }} \kappa$ by Theorem 4.6.

REMARK 4.16 (Isometry between $s$-block GLT sequences and $s \times s$ matrix-valued measurable functions). With the notation used in Definition 4.5, suppose we identify two $s$-block matrix-sequences $\left\{A_{n}\right\}_{n},\left\{B_{n}\right\}_{n} \in \mathscr{E}^{(s)}$ whenever their difference $\left\{A_{n}-B_{n}\right\}_{n}$ is zero-distributed and two measurable functions $\kappa, \xi \in \mathfrak{M}^{(s)}$ whenever their difference $\kappa-\xi$ equals $O_{s}$ a.e. Let $\mathscr{G}^{(s)} \subset \mathscr{E}^{(s)}$ be the subspace of $\mathscr{E}^{(s)}$ consisting of $s$-block GLT sequences and consider the application $\mathbb{J}$ from $\mathscr{G}^{(s)}$ to $\mathfrak{M}^{(s)}$ that associates with each $\left\{A_{n}\right\}_{n} \in \mathscr{G}^{(s)}$ its symbol $\kappa \in \mathfrak{M}^{(s)}$. This application is well-defined by Proposition 4.9. Moreover, if $\left\{A_{n}\right\}_{n} \sim_{\mathrm{GLT}} \kappa$, then $\left\{B_{n}\right\}_{n} \sim_{\mathrm{GLT}} \kappa$ is equivalent to $\left\{A_{n}-B_{n}\right\}_{n} \sim_{\sigma} 0$ by Remark 4.4 and Theorems 3.8 and 4.7. This means that, after identifying two $s$-block matrix-sequences $\left\{A_{n}\right\}_{n},\left\{B_{n}\right\}_{n}$ whenever $\left\{A_{n}-B_{n}\right\}_{n} \sim_{\sigma} 0$, the application $\mathbb{J}$ is still well-defined and, moreover, it is injective. By Theorem 4.15, $\mathbb{J}$ is also surjective. Finally, by Theorems 2.32 and 4.7, given any gauge function $\varphi$, if $\left\{A_{n}\right\}_{n} \sim_{\mathrm{GLT}} \kappa$ and $\left\{B_{n}\right\}_{n} \sim_{\mathrm{GLT}} \xi$, then

$$
d_{\text {a.c.s. }}^{\varphi}\left(\left\{A_{n}\right\}_{n},\left\{B_{n}\right\}_{n}\right)=p_{\text {a.c.s. }}^{\varphi}\left(\left\{A_{n}-B_{n}\right\}_{n}\right)=p_{\text {measure }}^{\varphi}(\kappa-\xi)=d_{\text {measure }}^{\varphi}(\kappa, \xi),
$$

which means that $\mathbb{J}$ is an isometry with respect to $d_{\text {a.c.s. }}^{\varphi}$ on $\mathscr{G}^{(s)}$ and $d_{\text {measure }}^{\varphi}$ on $\mathfrak{M}^{(s)}$.

4.4. The block GLT algebra. The next theorems are of fundamental importance. In particular, the first one shows that the set of $s$-block GLT pairs $\mathcal{G}^{(s)}$ defined in (4.1) is a *-subalgebra of $\mathscr{E}^{(s)} \times \mathfrak{M}^{(s)}$.

THEOREM 4.17. Let $\left\{A_{n}\right\}_{n} \sim_{\mathrm{GLT}} \kappa$ and $\left\{B_{n}\right\}_{n} \sim_{\mathrm{GLT}} \xi$. Then:

1. $\left\{A_{n}^{*}\right\}_{n} \sim_{\mathrm{GLT}} \kappa^{*}$;

2. $\left\{\alpha A_{n}+\beta B_{n}\right\}_{n} \sim_{\text {GLT }} \alpha \kappa+\beta \xi$ for all $\alpha, \beta \in \mathbb{C}$;

3. $\left\{A_{n} B_{n}\right\}_{n} \sim_{\mathrm{GLT}} \kappa \xi$.

Proof. The first two statements have already been settled before (see Remark 4.4). We prove the third one. By Definition 4.5, there exist $\left(\left\{A_{n, m}\right\}_{n}, \kappa_{m}\right),\left(\left\{B_{n, m}\right\}_{n}, \xi_{m}\right) \in \mathcal{A}^{(s)}$ such that $\left(\left\{A_{n, m}\right\}_{n}, \kappa_{m}\right) \rightarrow\left(\left\{A_{n}\right\}_{n}, \kappa\right)$ and $\left(\left\{B_{n, m}\right\}_{n}, \xi_{m}\right) \rightarrow\left(\left\{B_{n}\right\}_{n}, \xi\right)$ in the space $\left(\mathscr{E}^{(s)} \times \mathfrak{M}^{(s)}, \tau_{\text {a.c.s. }} \times \tau_{\text {measure }}\right)$, i.e.:

- $\left\{A_{n, m}\right\}_{n} \stackrel{\text { a.c.s. }}{\longrightarrow}\left\{A_{n}\right\}_{n}$ and $\left\{B_{n, m}\right\}_{n} \stackrel{\text { a.c.s. }}{\longrightarrow}\left\{B_{n}\right\}_{n}$;

- $\kappa_{m} \rightarrow \kappa$ in measure and $\xi_{m} \rightarrow \xi$ in measure.

Considering that every block GLT sequence is s.u. (see Remark 4.8), from Theorem 2.36 and Lemma 2.4, we obtain:

- $\left\{A_{n, m} B_{n, m}\right\}_{n} \stackrel{\text { a.c.s. }}{\longrightarrow}\left\{A_{n} B_{n}\right\}_{n}$;

- $\kappa_{m} \xi_{m} \rightarrow \kappa \xi$ in measure.

Since $\left(\left\{A_{n, m} B_{n, m}\right\}_{n}, \kappa_{m} \xi_{m}\right) \in \mathcal{A}^{(s)}$, by definition we have $\left\{A_{n} B_{n}\right\}_{n} \sim_{\mathrm{GLT}} \kappa \xi$.

THEOREM 4.18. If $\left\{A_{n}\right\}_{n} \sim_{\mathrm{GLT}} \kappa$ and $\kappa$ is invertible a.e., then $\left\{A_{n}^{\dagger}\right\}_{n} \sim_{\mathrm{GLT}} \kappa^{-1}$.

Proof. Since $\kappa$ is measurable and invertible a.e., its inverse $\kappa^{-1}$ is a well-defined measurable function. Thus, by Theorem 4.15 , there exists an $s$-block GLT sequence $\left\{B_{n}\right\}_{n}$ 
such that $\left\{B_{n}\right\}_{n} \sim_{\mathrm{GLT}} \kappa^{-1}$. By Theorem 4.17 we have $\left\{B_{n} A_{n}-I_{s n}\right\}_{n} \sim_{\mathrm{GLT}} \kappa^{-1} \kappa-I_{s}$, which implies $\left\{B_{n} A_{n}-I_{s n}\right\}_{n} \sim_{\sigma} 0$ by Theorem 4.7, since $\kappa^{-1} \kappa-I_{s}=O_{s}$ a.e. Hence, $\left\{B_{n} A_{n}\right\}_{n} \stackrel{\text { a.c.s. }}{\longrightarrow}\left\{I_{s n}\right\}_{n}$. Since $\kappa$ is invertible a.e., $\left\{A_{n}\right\}_{n}$ is s.v. by Theorem 4.7 and Proposition 2.25. It follows that $A_{n}^{\dagger}$ is s.u. (see Remark 2.23), and so, by Theorem 2.36, $\left\{B_{n} A_{n} A_{n}^{\dagger}\right\}_{n} \stackrel{\text { a.c.s. }}{\longrightarrow}\left\{A_{n}^{\dagger}\right\}_{n}$, i.e.,

$$
\left\{B_{n} A_{n} A_{n}^{\dagger}-A_{n}^{\dagger}\right\}_{n} \sim_{\sigma} 0 .
$$

Now we observe that, by definition of $A_{n}^{\dagger}$,

$$
A_{n} A_{n}^{\dagger}=I_{s n}+S_{n}, \quad \operatorname{rank}\left(S_{n}\right)=\#\left\{i \in\{1, \ldots, s n\}: \sigma_{i}\left(A_{n}\right)=0\right\} .
$$

Considering that $\left\{A_{n}\right\}_{n}$ is s.v., we have

$$
\lim _{n \rightarrow \infty} \frac{\operatorname{rank}\left(S_{n}\right)}{n}=0
$$

Hence, from (4.2) we obtain

$$
\left\{B_{n}+Z_{n}-A_{n}^{\dagger}\right\}_{n} \sim_{\sigma} 0,
$$

where $Z_{n}=B_{n} S_{n}$ is zero-distributed by Theorem 2.16. Thus, $A_{n}^{\dagger}=B_{n}+Z_{n}$, and it follows from Theorems 3.8 and 4.17 that $\left\{A_{n}^{\dagger}\right\}_{n} \sim_{\mathrm{GLT}} \kappa^{-1}$.

THEOREM 4.19. If $\left\{A_{n}\right\}_{n} \sim_{\mathrm{GLT}} \kappa$ and each $A_{n}$ is Hermitian, then $\left\{f\left(A_{n}\right)\right\}_{n} \sim_{\mathrm{GLT}} f(\kappa)$ for any continuous function $f: \mathbb{C} \rightarrow \mathbb{C}$.

Proof. Since every $A_{n}$ is Hermitian by assumption and $\kappa$ is Hermitian a.e. by Proposition 4.10, it suffices to prove the theorem for real continuous functions $f: \mathbb{R} \rightarrow \mathbb{R}$. Indeed, suppose we have proved the theorem for this kind of functions, and let $f: \mathbb{C} \rightarrow \mathbb{C}$ be any continuous complex function. Denote by $\alpha, \beta: \mathbb{R} \rightarrow \mathbb{R}$ the real and imaginary parts of the restriction of $f$ to $\mathbb{R}$. Then, $\alpha, \beta$ are continuous functions such that $f(x)=\alpha(x)+\mathrm{i} \beta(x)$ for all $x \in \mathbb{R}$, and since the eigenvalues of $A_{n}$ are real, we have $f\left(A_{n}\right)=\alpha\left(A_{n}\right)+\mathrm{i} \beta\left(A_{n}\right)$. In view of the relations $\left\{\alpha\left(A_{n}\right)\right\}_{n} \sim_{\mathrm{GLT}} \alpha(\kappa)$ and $\left\{\beta\left(A_{n}\right)\right\}_{n} \sim_{\mathrm{GLT}} \beta(\kappa)$, Theorem 4.17 yields $\left\{f\left(A_{n}\right)\right\}_{n} \sim_{\text {GLT }} \alpha(\kappa)+\mathrm{i} \beta(\kappa)$, and so $\left\{A_{n}\right\}_{n} \sim_{\text {GLT }} f(\kappa)$ because $\alpha(\kappa)+\mathrm{i} \beta(\kappa)=f(\kappa)$ a.e. as $\kappa$ is Hermitian a.e.

Let $f: \mathbb{R} \rightarrow \mathbb{R}$ be a real continuous function. For each $M>0$, let $\left\{p_{m, M}\right\}_{m}$ be a sequence of polynomials that converges uniformly to $f$ over $[-M, M]$ :

$$
\lim _{m \rightarrow \infty}\left\|f-p_{m, M}\right\|_{\infty,[-M, M]}=0 .
$$

Note that such a sequence exists by the Weierstrass theorem; see, e.g., [70, Theorem 7.26]. By replacing $p_{m, M}$ with $p_{m, M}+f(0)-p_{m, M}(0)$ if necessary, we may assume, without loss of generality, that $p_{m, M}(0)=f(0)$. Since any block GLT sequence is s.u. (by Remark 4.8), the sequence $\left\{A_{n}\right\}_{n}$ is s.u. Hence, by Remark 2.21, for all $M>0$ there exists $n_{M}$ such that, for $n \geq n_{M}$,

$$
A_{n}=\hat{A}_{n, M}+\tilde{A}_{n, M}, \quad \operatorname{rank}\left(\hat{A}_{n, M}\right) \leq r(M) n, \quad\left\|\tilde{A}_{n, M}\right\| \leq M,
$$

where $r(M) \rightarrow 0$ as $M \rightarrow \infty$, the matrices $\hat{A}_{n, M}$ and $\tilde{A}_{n, M}$ are Hermitian, and for all functions $g: \mathbb{R} \rightarrow \mathbb{R}$ satisfying $g(0)=0$, we have

$$
g\left(\hat{A}_{n, M}+\tilde{A}_{n, M}\right)=g\left(\hat{A}_{n, M}\right)+g\left(\tilde{A}_{n, M}\right) .
$$


Taking into account that $\left(f-p_{m, M}\right)(0)=0$, for every $M>0$, every $m$ and every $n \geq n_{M}$, we can write

$$
\begin{aligned}
f\left(A_{n}\right) & =p_{m, M}\left(A_{n}\right)+f\left(A_{n}\right)-p_{m, M}\left(A_{n}\right) \\
& =p_{m, M}\left(A_{n}\right)+\left(f-p_{m, M}\right)\left(\hat{A}_{n, M}\right)+\left(f-p_{m, M}\right)\left(\tilde{A}_{n, M}\right) \\
& =p_{m, M}\left(A_{n}\right)+R_{n, m, M}+N_{n, m, M},
\end{aligned}
$$

where, in view of (4.3), the matrices

$$
R_{n, m, M}=\left(f-p_{m, M}\right)\left(\hat{A}_{n, M}\right), \quad N_{n, m, M}=\left(f-p_{m, M}\right)\left(\tilde{A}_{n, M}\right)
$$

satisfy

$$
\begin{aligned}
\operatorname{rank}\left(R_{n, m, M}\right) & \leq \operatorname{rank}\left(\hat{A}_{n, M}\right) \leq r(M) n, \\
\left\|N_{n, m, M}\right\| & \leq\left\|f-p_{m, M}\right\|_{\infty,[-M, M]} .
\end{aligned}
$$

Choose a sequence $\left\{M_{m}\right\}_{m}$ such that

$$
\begin{aligned}
M_{m} \rightarrow \infty, & \left\|f-p_{m, M_{m}}\right\|_{\infty,\left[-M_{m}, M_{m}\right]} \rightarrow 0 . \\
f\left(A_{n}\right) & =p_{m, M_{m}}\left(A_{n}\right)+R_{n, m, M_{m}}+N_{n, m, M_{m}}, \\
\operatorname{rank}\left(R_{n, m, M_{m}}\right) \leq & r\left(M_{m}\right) n, \\
\left\|N_{n, m, M_{m}}\right\| \leq & \leq f-p_{m, M_{m}} \|_{\infty,\left[-M_{m}, M_{m}\right]},
\end{aligned}
$$

which implies that

$$
\left\{p_{m, M_{m}}\left(A_{n}\right)\right\}_{n} \stackrel{\text { a.c.s. }}{\longrightarrow}\left\{f\left(A_{n}\right)\right\}_{n} .
$$

Moreover, by Theorem 4.17,

$$
\left\{p_{m, M_{m}}\left(A_{n}\right)\right\}_{n} \sim_{\mathrm{GLT}} p_{m, M_{m}}(\kappa) .
$$

Finally, by (4.4),

$$
\begin{aligned}
\left\|f(\kappa)-p_{m, M_{m}}(\kappa)\right\| & =\max _{i=1, \ldots, s}\left|\left(f-p_{m, M_{m}}\right)\left(\lambda_{i}(\kappa)\right)\right| \\
& \leq\left\|f-p_{m, M_{m}}\right\|_{\infty,[-\|\kappa\|,\|\kappa\|]} \rightarrow 0 \text { a.e., }
\end{aligned}
$$

which implies that

$$
p_{m, M_{m}}(\kappa) \rightarrow f(\kappa) \text { a.e. }
$$

All the hypotheses of Theorem 4.6 are then satisfied and $\left\{f\left(A_{n}\right)\right\}_{n} \sim_{\text {GLT }} f(\kappa)$.

4.5. Topological density results for block GLT sequences. Theorem 4.6 admits the following simple but important converse, which will allow us to prove the main result of this section (Theorem 4.21).

THEOREM 4.20. Let $\left\{A_{n}\right\}_{n} \sim_{\text {GLT }} \kappa$ and $\left\{B_{n, m}\right\}_{n} \sim_{\text {GLT }} \kappa_{m}$ for every $m$. Then

$$
\left\{B_{n, m}\right\}_{n} \stackrel{\text { a.c.s. }}{\longrightarrow}\left\{A_{n}\right\}_{n} \Longleftrightarrow \kappa_{m} \rightarrow \kappa \text { in measure. }
$$

Proof. By Remark 4.16,

$$
d_{\text {a.c.s. }}\left(\left\{A_{n}\right\}_{n},\left\{B_{n, m}\right\}_{n}\right)=d_{\text {measure }}\left(\kappa, \kappa_{m}\right),
$$

and the proof is complete.

THEOREM 4.21. Let $\left\{A_{n}\right\}_{n} \sim_{\mathrm{GLT}} \kappa$. Then, for all functions $a_{i, m}, f_{i, m}, i=1, \ldots, N_{m}$, such that 
1. $a_{i, m}:[0,1] \rightarrow \mathbb{C}^{s \times s}$ is Riemann-integrable and $f_{i, m} \in L^{1}([-\pi, \pi], s)$,

2. $\sum_{i=1}^{N_{m}} a_{i, m}(x) f_{i, m}(\theta) \rightarrow \kappa(x, \theta)$ in measure, we have $\left\{\sum_{i=1}^{N_{m}} D_{n}\left(a_{i, m}\right) T_{n}\left(f_{i, m}\right)\right\}_{n} \stackrel{\text { a.c.s. }}{\longrightarrow}\left\{A_{n}\right\}_{n}$. In particular, $\left\{A_{n}\right\}_{n}$ admits an a.c.s. of the form

$$
\begin{aligned}
& \left\{\left\{\sum_{\alpha, \beta=1}^{s} \sum_{j=-N_{m}}^{N_{m}} D_{n, s}\left(a_{\alpha \beta, j}^{(m)}\right) T_{n}\left(e^{\mathrm{i} j \theta} E_{\alpha \beta}^{(s)}\right)\right\}_{n}\right\}_{m} \\
& =\left\{\left\{\sum_{j=-N_{m}}^{N_{m}} D_{n}\left(a_{j}^{(m)}\right) T_{n}\left(e^{\mathrm{i} j \theta} I_{s}\right)\right\}_{n}\right\}_{m},
\end{aligned}
$$

where $N_{m} \in \mathbb{N}, a_{\alpha \beta, j}^{(m)}:[0,1] \rightarrow \mathbb{C}$ belongs to $C^{\infty}([0,1])$,

$$
a_{j}^{(m)}=\sum_{\alpha, \beta=1}^{s} a_{\alpha \beta, j}^{(m)} E_{\alpha \beta}^{(s)}=\left[a_{\alpha \beta, j}^{(m)}\right]_{\alpha, \beta=1}^{s},
$$

and

$$
\sum_{\alpha, \beta=1}^{s} \sum_{j=-N_{m}}^{N_{m}} a_{\alpha \beta, j}^{(m)}(x) e^{\mathrm{i} j \theta} E_{\alpha \beta}^{(s)}=\sum_{j=-N_{m}}^{N_{m}} a_{j}^{(m)}(x) e^{\mathrm{i} j \theta} \rightarrow \kappa(x, \theta) \text { a.e. }
$$

Proof. Let $a_{i, m}, f_{i, m}, i=1, \ldots, N_{m}$, be functions with the properties specified in the statement of the theorem. Then

$$
\left\{\sum_{i=1}^{N_{m}} D_{n}\left(a_{i, m}\right) T_{n}\left(f_{i, m}\right)\right\}_{n} \sim_{\mathrm{GLT}} \sum_{i=1}^{N_{m}} a_{i, m}(x) f_{i, m}(\theta)
$$

by Remark 4.3 and Theorems 3.12 and 4.17. Therefore, the convergence

$$
\left\{\sum_{i=1}^{N_{m}} D_{n}\left(a_{i, m}\right) T_{n}\left(f_{i, m}\right)\right\}_{n} \stackrel{\text { a.c.s. }}{\longrightarrow}\left\{A_{n}\right\}_{n}
$$

follows from Theorem 4.20 applied with

$$
B_{n, m}=\sum_{i=1}^{N_{m}} D_{n}\left(a_{i, m}\right) T_{n}\left(f_{i, m}\right), \quad \kappa_{m}(x, \theta)=\sum_{i=1}^{N_{m}} a_{i, m}(x) f_{i, m}(\theta) .
$$

To obtain for $\left\{A_{n}\right\}_{n}$ an a.c.s. of the form (4.5), we use the result of this theorem in combination with [50, Lemma 2.8]. The details are as follows. By [50, Lemma 2.8], for every $\alpha, \beta=1, \ldots, s$, there exists a sequence of measurable functions $\kappa_{m, \alpha \beta}:[0,1] \times[-\pi, \pi] \rightarrow \mathbb{C}$ such that $\kappa_{m, \alpha \beta}$ is of the form

$$
\kappa_{m, \alpha \beta}(x, \theta)=\sum_{j=-N_{m}}^{N_{m}} a_{\alpha \beta, j}^{(m)}(x) e^{\mathrm{i} j \theta},
$$

with $N_{m} \in \mathbb{N}$ and $a_{\alpha \beta, j}^{(m)}:[0,1] \rightarrow \mathbb{C}$ belonging to $C^{\infty}([0,1])$ and $\kappa_{m, \alpha \beta} \rightarrow \kappa_{\alpha \beta}$ a.e. Then, $\sum_{\alpha, \beta=1}^{s} \kappa_{m, \alpha \beta} E_{\alpha \beta}^{(s)} \rightarrow \sum_{\alpha, \beta=1}^{s} \kappa_{\alpha \beta} E_{\alpha \beta}^{(s)}=\kappa$ a.e., and so, by the result of this theorem,

$$
\left\{\sum_{\alpha, \beta=1}^{s} \sum_{j=-N_{m}}^{N_{m}} D_{n}\left(a_{\alpha \beta, j}^{(m)} I_{s}\right) T_{n}\left(e^{\mathrm{i} j \theta} E_{\alpha \beta}^{(s)}\right)\right\}_{n} \stackrel{\text { a.c.s. }}{\longrightarrow}\left\{A_{n}\right\}_{n} .
$$


REMARK 4.22 (Topological density in the space of block GLT sequences). With the notation introduced in Section 4.1, we recall that the set of $s$-block GLT pairs

$$
\mathcal{G}^{(s)}=\left\{\left(\left\{A_{n}\right\}_{n}, \kappa\right) \in \mathscr{E}^{(s)} \times \mathfrak{M}^{(s)}:\left\{A_{n}\right\}_{n} \sim_{\mathrm{GLT}} \kappa\right\}
$$

is closed in $\left(\mathscr{E}^{(s)} \times \mathfrak{M}^{(s)}, \tau_{\text {a.c.s. }} \times \tau_{\text {measure }}\right)$ by Definition 4.5 . Consider the subset of $\mathcal{G}^{(s)}$ consisting of the $s$-block GLT pairs of the form

$$
\left(\sum_{i=1}^{N} D_{n, s}\left(a_{i}\right) T_{n}\left(f_{i}\right), \sum_{i=1}^{N} a_{i}(x) f_{i}(\theta)\right),
$$

where $a_{i}:[0,1] \rightarrow \mathbb{C}$ belongs to $C^{\infty}([0,1]), f_{i}$ is a trigonometric monomial in $\left\{e^{\mathrm{i} j \theta} E_{\alpha \beta}^{(s)}:\right.$ $j \in \mathbb{Z}, 1 \leq \alpha, \beta \leq s\}$ for all $i=1, \ldots, N$, and $N \in \mathbb{N}$. Then, according to Theorem 4.21, this subset is dense in $\mathcal{G}^{(s)}$, i.e., its closure in $\left(\mathscr{E}(s) \times \mathfrak{M}^{(s)}, \tau_{\text {a.c.s. }} \times \tau_{\text {measure }}\right)$ coincides precisely with $\mathcal{G}^{(s)}$.

4.6. Characterizations of block GLT sequences. The next result is a characterization theorem for block GLT sequences. All the provided characterizations have already been proved before, but it is anyway useful to collect them in a single statement.

THEOREM 4.23. Let $\left\{A_{n}\right\}_{n}$ be an s-block matrix-sequence, and let $\kappa:[0,1] \times[-\pi, \pi] \rightarrow$ $\mathbb{C}^{s \times s}$ be a measurable function. The following conditions are equivalent.

1. $\left\{A_{n}\right\}_{n} \sim_{\mathrm{GLT}} \kappa$.

2. For all sequences $\left\{\kappa_{m}\right\}_{m},\left\{\left\{B_{n, m}\right\}_{n}\right\}_{m}$ such that

- $\left\{B_{n, m}\right\}_{n} \sim_{\mathrm{GLT}} \kappa_{m}$ for every $m$,

- $\kappa_{m} \rightarrow \kappa$ in measure,

we have $\left\{B_{n, m}\right\}_{n} \stackrel{\text { a.c.s. }}{\longrightarrow}\left\{A_{n}\right\}_{n}$.

3. There exist functions $a_{i, m}, f_{i, m}, i=1, \ldots, N_{m}$, such that

- $a_{i, m}:[0,1] \rightarrow \mathbb{C}$ belongs to $C^{\infty}([0,1])$ and $f_{i, m}$ is a trigonometric monomial belonging to $\left\{e^{\mathrm{i} j \theta} E_{\alpha \beta}^{(s)}: j \in \mathbb{Z}, 1 \leq \alpha, \beta \leq s\right\}$,

- $\sum_{i=1}^{N_{m}} a_{i, m}(x) f_{i, m}(\theta) \rightarrow \kappa(x, \theta)$ a.e.,

- $\left\{\sum_{i=1}^{N_{m}} D_{n, s}\left(a_{i, m}\right) T_{n}\left(f_{i, m}\right)\right\}_{n} \stackrel{\text { a.c.s. }}{\longrightarrow}\left\{A_{n}\right\}_{n}$.

4. There exist sequences $\left\{\kappa_{m}\right\}_{m},\left\{\left\{B_{n, m}\right\}_{n}\right\}_{m}$ such that

- $\left\{B_{n, m}\right\}_{n} \sim_{\mathrm{GLT}} \kappa_{m}$ for every $m$,

- $\kappa_{m} \rightarrow \kappa$ in measure,

- $\left\{B_{n, m}\right\}_{n} \stackrel{\text { a.c.s. }}{\longrightarrow}\left\{A_{n}\right\}_{n}$.

Proof. The implication $1 \Longrightarrow 2$ follows from Theorem 4.20 . The implication $2 \Longrightarrow 3$ follows from the observation that we can find functions $a_{i, m}, f_{i, m}, i=1, \ldots, N_{m}$, with the first two properties specified in item 3 (by [50, Lemma 2.8], as we have already seen in the proof of Theorem 4.21) and, moreover, $\left\{\sum_{i=1}^{N_{m}} D_{n, s}\left(a_{i, m}\right) T_{n}\left(f_{i, m}\right)\right\}_{n} \sim_{\mathrm{GLT}} \sum_{i=1}^{N_{m}} a_{i, m}(x) f_{i, m}(\theta)$ (by Theorems 3.18 and 4.17). The implication $3 \Longrightarrow 4$ is obvious (it suffices to take $B_{n, m}=\sum_{i=1}^{N_{m}} D_{n, s}\left(a_{i, m}\right) T_{n}\left(f_{i, m}\right)$ and $\left.\kappa_{m}(x, \theta)=\sum_{i=1}^{N_{m}} a_{i, m}(x) f_{i, m}(\theta)\right)$. Finally, the implication $4 \Longrightarrow 1$ is Theorem 4.6.

4.7. Sequences of block diagonal sampling matrices. We have encountered in Section 3.3 and Remark 4.3 the three most important examples of block GLT sequences, i.e., zerodistributed sequences, block Toeplitz sequences, and sequences of block diagonal sampling matrices. Concerning the latter kind of sequences, we have proved that $\left\{D_{n}(a)\right\}_{n} \sim_{\text {GLT }} a(x)$ whenever $a:[0,1] \rightarrow \mathbb{C}^{s \times s}$ is Riemann-integrable. From a mathematical point of view, 
however, the GLT relation $\left\{D_{n}(a)\right\}_{n} \sim_{\mathrm{GLT}} a(x)$ makes sense for all measurable functions $a:[0,1] \rightarrow \mathbb{C}^{s \times s}$, and it is therefore natural to ask whether we can drop the Riemannintegrability assumption. In Theorem 4.24 we show that the relation $\left\{D_{n}(a)\right\}_{n} \sim_{\text {GLT }} a(x)$ holds for all functions $a:[0,1] \rightarrow \mathbb{C}^{s \times s}$ that are continuous a.e. in $[0,1]$. Since a function $a:[0,1] \rightarrow \mathbb{C}^{s \times s}$ is Riemann-integrable if and only if $a$ is bounded and continuous a.e. (see Section 2.3.5), Theorem 4.24 is an extension of both Theorem 3.11 and Remark 4.3. More precisely, in Theorem 4.24 we are dropping the boundedness assumption.

THEOREM 4.24. If $a:[0,1] \rightarrow \mathbb{C}^{s \times s}$ is continuous a.e., then $\left\{D_{n}(a)\right\}_{n} \sim_{\mathrm{GLT}} a(x)$.

Proof. By looking at the decomposition of $D_{n}(a)$ considered in Remark 4.3, it is immediately clear that, in order to prove the theorem, it is enough to show that

$$
\left\{D_{n, s}(a)\right\}_{n} \sim_{\mathrm{GLT}} a(x) I_{s}
$$

whenever $a:[0,1] \rightarrow \mathbb{C}$ is a scalar a.e. continuous function. Moreover, for an arbitrary a.e. continuous function $a:[0,1] \rightarrow \mathbb{C}$, we can write $a=\alpha_{+}-\alpha_{-}+\mathrm{i} \beta_{+}-\mathrm{i} \beta_{-}$, where $\alpha_{ \pm}, \beta_{ \pm}:[0,1] \rightarrow \mathbb{R}$ are nonnegative a.e. continuous functions; simply take

$$
\begin{aligned}
\alpha_{+} & =\max (\Re(a), 0), & \alpha_{-} & =-\min (\Re(a), 0), \\
\beta_{+} & =\max (\Im(a), 0), & \beta_{-} & =-\min (\Im(a), 0) .
\end{aligned}
$$

Hence, by Theorem 4.17 and the linearity of $D_{n, s}(a)$ with respect to its argument $a$, it suffices to prove the relation $\left\{D_{n, s}(a)\right\}_{n} \sim_{\mathrm{GLT}} a(x)$ in the case where $a:[0,1] \rightarrow \mathbb{R}$ is a nonnegative a.e. continuous function.

Let $a:[0,1] \rightarrow[0, \infty)$ be a nonnegative a.e. continuous function. Denote by $a_{m}$ the truncation of $a$ at level $m$, i.e.,

$$
a_{m}(x)= \begin{cases}a(x), & \text { if } a(x) \leq m, \\ m, & \text { if } a(x)>m .\end{cases}
$$

Since $a_{m}$ is bounded and continuous a.e., $a_{m}$ is Riemann-integrable, hence

$$
\left\{D_{n, s}\left(a_{m}\right)\right\}_{n} \sim_{\mathrm{GLT}} a_{m}(x) I_{s}
$$

by Theorem 3.11. Moreover, it is clear that $a_{m} \rightarrow a$ pointwise, so

$$
a_{m} \rightarrow a \text { in measure. }
$$

We show that

$$
\left\{D_{n, s}\left(a_{m}\right)\right\}_{n} \stackrel{\text { a.c.s. }}{\longrightarrow}\left\{D_{n, s}(a)\right\}_{n},
$$

after which the application of Theorem 4.6 concludes the proof. In order to show that $\left\{D_{n, s}\left(a_{m}\right)\right\}_{n} \stackrel{\text { a.c.s. }}{\longrightarrow}\left\{D_{n, s}(a)\right\}_{n}$, we observe that $D_{n, s}\left(a_{m}\right)=D_{n}\left(a_{m}\right) \otimes I_{s}$ and $D_{n, s}(a)=D_{n}(a) \otimes I_{s}$. Therefore, since it was proved in [50, pp. 153-154] that $\left\{D_{n}\left(a_{m}\right)\right\}_{n} \stackrel{\text { a.c.s. }}{\longrightarrow}\left\{D_{n}(a)\right\}_{n}$, it is immediately clear from the definition of a.c.s. and the properties of tensor products that $\left\{D_{n, s}\left(a_{m}\right)\right\}_{n} \stackrel{\text { a.c.s. }}{\longrightarrow}\left\{D_{n, s}(a)\right\}_{n}$.

4.8. Sequences of block matrices with block GLT blocks. This section is devoted to proving the following theorem, which says that, up to a suitable permutation transformation, a sequence of block matrices with block GLT blocks is a block GLT sequence whose symbol is obtained by "putting together" the symbols of each block GLT block. Recall that $\Pi_{n, s, r}$ denotes the special permutation matrix (2.1).

THEOREM 4.25. For $i, j=1, \ldots, s$, let $\left\{A_{n, i j}\right\}_{n}$ be an $r$-block GLT sequence with symbol $\kappa_{i j}:[0,1] \times[-\pi, \pi] \rightarrow \mathbb{C}^{r \times r}$. Set $A_{n}=\left[A_{n, i j}\right]_{i, j=1}^{s}$ and $\kappa=\left[\kappa_{i j}\right]_{i, j=1}^{s}$. Then, $\left\{\Pi_{n, s, r} A_{n} \Pi_{n, s, r}^{T}\right\}_{n}$ is an rs-block GLT sequence with symbol $\kappa$. 
Proof. The proof consists of the following two steps.

Step 1. We first prove the theorem under the additional assumption that $A_{n, i j}$ is of the form

$$
A_{n, i j}=\sum_{\ell=1}^{L_{i j}} D_{n}\left(a_{\ell, i j}\right) T_{n}\left(f_{\ell, i j}\right),
$$

where $L_{i j} \in \mathbb{N}, a_{\ell, i j}:[0,1] \rightarrow \mathbb{C}^{r \times r}$ is continuous a.e., and $f_{\ell, i j}:[-\pi, \pi] \rightarrow \mathbb{C}^{r \times r}$ belongs to $L^{1}([-\pi, \pi], r)$. Note that the symbol of $\left\{A_{n, i j}\right\}_{n}$ is

$$
\kappa_{i j}(x, \theta)=\sum_{\ell=1}^{L_{i j}} a_{\ell, i j}(x) f_{\ell, i j}(\theta) .
$$

By setting $L=\max _{i, j=1, \ldots, s} L_{i j}$ and adding zero matrices of the form $D_{n}\left(O_{r}\right) T_{n}\left(O_{r}\right)$ in the summation (4.7) whenever $L_{i j}<L$, we can assume, without loss of generality, that

$$
A_{n, i j}=\sum_{\ell=1}^{L} D_{n}\left(a_{\ell, i j}\right) T_{n}\left(f_{\ell, i j}\right), \quad \kappa_{i j}(x, \theta)=\sum_{\ell=1}^{L} a_{\ell, i j}(x) f_{\ell, i j}(\theta),
$$

with $L$ independent of $i, j$. Then,

$$
\begin{aligned}
\Pi_{n, s, r} A_{n} \Pi_{n, s, r}^{T} & =\sum_{\ell=1}^{L} \Pi_{n, s, r}\left[D_{n}\left(a_{\ell, i j}\right) T_{n}\left(f_{\ell, i j}\right)\right]_{i, j=1}^{s} \Pi_{n, s, r}^{T} \\
& =\sum_{\ell=1}^{L} \Pi_{n, s, r}\left[\sum_{i, j=1}^{s} E_{i j}^{(s)} \otimes D_{n}\left(a_{\ell, i j}\right) T_{n}\left(f_{\ell, i j}\right)\right] \Pi_{n, s, r}^{T} \\
& =\sum_{\ell=1}^{L} \Pi_{n, s, r}\left[\sum_{i, j=1}^{s}\left(E_{i j}^{(s)} \otimes D_{n}\left(a_{\ell, i j}\right)\right)\left(I_{s} \otimes T_{n}\left(f_{\ell, i j}\right)\right)\right] \Pi_{n, s, r}^{T} \\
& =\sum_{\ell=1}^{L} \sum_{i, j=1}^{s} \Pi_{n, s, r}\left(E_{i j}^{(s)} \otimes D_{n}\left(a_{\ell, i j}\right)\right) \Pi_{n, s, r}^{T} \Pi_{n, s, r}\left(I_{s} \otimes T_{n}\left(f_{\ell, i j}\right)\right) \Pi_{n, s, r}^{T}
\end{aligned}
$$

By Theorems 2.42 and 2.43,

$$
\begin{aligned}
\Pi_{n, s, r}\left(E_{i j}^{(s)} \otimes D_{n}\left(a_{\ell, i j}\right)\right) \Pi_{n, s, r}^{T} & =D_{n}\left(E_{i j}^{(s)} \otimes a_{\ell, i j}\right), \\
\Pi_{n, s, r}\left(I_{s} \otimes T_{n}\left(f_{\ell, i j}\right)\right) \Pi_{n, s, r}^{T} & =T_{n}\left(I_{s} \otimes f_{\ell, i j}\right) .
\end{aligned}
$$

It follows that

$$
\Pi_{n, s, r} A_{n} \Pi_{n, s, r}^{T}=\sum_{\ell=1}^{L} \sum_{i, j=1}^{s} D_{n}\left(E_{i j}^{(s)} \otimes a_{\ell, i j}\right) T_{n}\left(I_{s} \otimes f_{\ell, i j}\right) .
$$

Thus, by Theorems 3.12, 4.17, and 4.24, $\left\{\Pi_{n, s, r} A_{n} \Pi_{n, s, r}^{T}\right\}_{n}$ is an $r s$-block GLT sequence with symbol

$$
\kappa(x, \theta)=\sum_{\ell=1}^{L} \sum_{i, j=1}^{s} E_{i j}^{(s)} \otimes a_{\ell, i j}(x) f_{\ell, i j}(\theta)=\left[\kappa_{i j}(x, \theta)\right]_{i, j=1}^{s} .
$$

Step 2. We now prove the theorem in its full generality. Since $\left\{A_{n, i j}\right\}_{n}$ is an $r$-block GLT sequence with symbol $\kappa_{i j}$, by Theorem 4.21 (applied with $s=r$ ), there exist functions $a_{\ell, i j}^{(m)}$, $f_{\ell, i j}^{(m)}, \ell=1, \ldots, L_{i j}^{(m)}$, such that 
- $a_{\ell, i j}^{(m)}:[0,1] \rightarrow \mathbb{C}^{r \times r}$ belongs to $C^{\infty}([0,1])$ and $f_{\ell, i j}^{(m)}:[-\pi, \pi] \rightarrow \mathbb{C}^{r \times r}$ is a trigonometric polynomial,

- $\kappa_{i j}^{(m)}(x, \theta)=\sum_{\ell=1}^{L_{i j}^{(m)}} a_{\ell, i j}^{(m)}(x) f_{\ell, i j}^{(m)}(\theta) \rightarrow \kappa_{i j}(x, \theta)$ a.e.,

- $\left\{A_{n, i j}^{(m)}=\sum_{\ell=1}^{L_{i j}^{(m)}} D_{n}\left(a_{\ell, i j}^{(m)}\right) T_{n}\left(f_{\ell, i j}^{(m)}\right)\right\}_{n} \stackrel{\text { a.c.s. }}{\longrightarrow}\left\{A_{n, i j}\right\}_{n}$.

Set $A_{n}^{(m)}=\left[A_{n, i j}^{(m)}\right]_{i, j=1}^{s}$ and $\kappa^{(m)}=\left[\kappa_{i j}^{(m)}\right]_{i, j=1}^{s}$. We have:

- $\left\{\Pi_{n, s, r} A_{n}^{(m)} \Pi_{n, s, r}^{T}\right\}_{n} \sim_{\mathrm{GLT}} \kappa^{(m)}$ by Step 1;

- $\kappa^{(m)} \rightarrow \kappa$ a.e. (and hence also in measure);

- $\left\{\Pi_{n, s, r} A_{n}^{(m)} \Pi_{n, s, r}^{T}\right\}_{n} \stackrel{\text { a.c.s. }}{\longrightarrow}\left\{\Pi_{n, s, r} A_{n} \Pi_{n, s, r}^{T}\right\}_{n}$ because $\left\{A_{n}^{(m)}\right\}_{n} \stackrel{\text { a.c.s. }}{\longrightarrow}\left\{A_{n}\right\}_{n}$ by Theorem 2.37.

We conclude that $\left\{\Pi_{n, s, r} A_{n} \Pi_{n, s, r}^{T}\right\}_{n} \sim_{\mathrm{GLT}} \kappa$ by Theorem 4.6.

4.9. Further possible definitions of block GLT sequences. In this section, we discuss a couple of possible alternative definitions of block GLT sequences. We will use the same notation as in Definition 4.5.

By Definition 4.5 and Theorem 4.17, the set of $s$-block GLT pairs

$$
\mathcal{G}^{(s)}=\left\{\left(\left\{A_{n}\right\}_{n}, \kappa\right):\left\{A_{n}\right\}_{n} \sim_{\mathrm{GLT}} \kappa\right\} \subseteq \mathscr{E}^{(s)} \times \mathfrak{M}^{(s)}
$$

is a closed *-subalgebra of $\mathscr{E}^{(s)} \times \mathfrak{M}^{(s)}$. By Theorems 3.8, 3.12, and 4.24, $\mathcal{G}^{(s)}$ contains the set

$$
\begin{aligned}
\mathcal{B}^{(s)}= & \left\{\left(\left\{T_{n}(f)\right\}_{n}, \kappa(x, \theta)=f(\theta)\right): f \in L^{1}([-\pi, \pi], s)\right\} \\
& \cup\left\{\left(\left\{D_{n}(a)\right\}_{n}, \kappa(x, \theta)=a(x)\right): a:[0,1] \rightarrow \mathbb{C}^{s \times s} \text { is continuous a.e. }\right\} \\
& \cup\left\{\left(\left\{Z_{n}\right\}_{n}, \kappa(x, \theta)=O_{s}\right):\left\{Z_{n}\right\}_{n} \sim_{\sigma} 0\right\} .
\end{aligned}
$$

By the results in Section 4.5, the algebra generated by $\mathcal{B}^{(s)}$ is dense in $\mathcal{G}^{(s)}$. In conclusion,

the set of s-block GLT pairs $\mathcal{G}^{(s)}$ is the closed ${ }^{*}$-subalgebra of $\mathscr{E}^{(s)} \times \mathfrak{M}^{(s)}$ generated by $\mathcal{B}^{(s)}$, i.e., the smallest closed ${ }^{*}$-subalgebra of $\mathscr{E}^{(s)} \times \mathfrak{M}^{(s)}$ containing $\mathcal{B}^{(s)}$.

Looking more carefully at the results in Section 4.5, we also note that, if we let

$$
\begin{aligned}
& \mathcal{C}^{(s)}=\{\left.\left(\left\{D_{n, s}(a)\right\}_{n}, \kappa(x, \theta)=a(x) I_{s}\right): a:[0,1] \rightarrow \mathbb{C} \text { belongs to } C^{\infty}([0,1])\right\} \\
& \cup\left\{\left(\left\{T_{n}\left(e^{\mathrm{i} j \theta} E_{\alpha \beta}^{(s)}\right)\right\}_{n}, \kappa(x, \theta)=e^{\mathrm{i} j \theta} E_{\alpha \beta}^{(s)}\right): j \in \mathbb{Z}, 1 \leq \alpha, \beta \leq s\right\},
\end{aligned}
$$

then

the set of s-block GLT pairs $\mathcal{G}^{(s)}$ is the closure of the subalgebra of $\mathscr{E}^{(s)} \times \mathfrak{M}^{(s)}$ generated by $\mathcal{C}^{(s)}$.

5. Summary of the theory. We conclude the theory of block GLT sequences by providing a self-contained summary, which contains everything one needs to know in order to understand the applications presented in the next chapter. It is assumed that anyone who reads this summary is aware of the notation and terminology used throughout this work, which will be only partially repeated here for the sake of brevity. The reader can find most of the notation and terminology in Section 2.1.

Matrix norms. Here is a list of important inequalities involving $p$-norms and Schatten $p$-norms of matrices.

N 1. $\|X\| \leq \sqrt{|X|_{1}|X|_{\infty}} \leq \max \left(|X|_{1},|X|_{\infty}\right)$ for all $X \in \mathbb{C}^{m \times m}$.

N2. $\|X\|_{1} \leq \operatorname{rank}(X)\|X\| \leq m\|X\|$ for all $X \in \mathbb{C}^{m \times m}$. 
N3. $\|X\|_{1} \leq \sum_{i, j=1}^{m}\left|x_{i j}\right|$ for all $X \in \mathbb{C}^{m \times m}$.

N4. $\|X\|_{2} \leq \sqrt{\|X\|\|X\|_{1}}$ for all $X \in \mathbb{C}^{m \times m}$.

Sequences of matrices and block matrix-sequences. A sequence of matrices is a sequence of the form $\left\{A_{n}\right\}_{n}$, where $A_{n}$ is a square matrix of size $d_{n}$ such that $d_{n} \rightarrow \infty$ as $n \rightarrow \infty$. If $\left\{A_{n}\right\}_{n}$ is a sequence of matrices with $A_{n}$ of size $d_{n}$, then we say that $\left\{A_{n}\right\}_{n}$ is sparsely unbounded (s.u.) if

$$
\lim _{M \rightarrow \infty} \limsup _{n \rightarrow \infty} \frac{\#\left\{i \in\left\{1, \ldots, d_{n}\right\}: \sigma_{i}\left(A_{n}\right)>M\right\}}{d_{n}}=0,
$$

and we say that $\left\{A_{n}\right\}_{n}$ is sparsely vanishing (s.v.) if

$$
\lim _{M \rightarrow \infty} \limsup _{n \rightarrow \infty} \frac{\#\left\{i \in\left\{1, \ldots, d_{n}\right\}: \sigma_{i}\left(A_{n}\right)<1 / M\right\}}{d_{n}}=0
$$

An $s$-block matrix-sequence is a sequence of matrices $\left\{A_{n}\right\}_{n}$ such that the size of the $n$th matrix is $d_{n}=s n$.

Singular value and eigenvalue distribution of a sequence of matrices. Let $\left\{A_{n}\right\}_{n}$ be a sequence of matrices with $A_{n}$ of size $d_{n}$, and let $f: D \subset \mathbb{R}^{k} \rightarrow \mathbb{C}^{r \times r}$ be a measurable function defined on a set $D$ with $0<\mu_{k}(D)<\infty$.

- We say that $\left\{A_{n}\right\}_{n}$ has a singular value distribution described by $f$, and we write $\left\{A_{n}\right\}_{n} \sim_{\sigma} f$, if

$$
\lim _{n \rightarrow \infty} \frac{1}{d_{n}} \sum_{i=1}^{d_{n}} F\left(\sigma_{i}\left(A_{n}\right)\right)=\frac{1}{\mu_{k}(D)} \int_{D} \frac{\sum_{i=1}^{r} F\left(\sigma_{i}(f(\mathbf{x}))\right)}{r} \mathrm{~d} \mathbf{x}, \quad \forall F \in C_{c}(\mathbb{R}) .
$$

In this case, $f$ is called the singular value symbol of $\left\{A_{n}\right\}_{n}$.

- We say that $\left\{A_{n}\right\}_{n}$ has a spectral (or eigenvalue) distribution described by $f$, and we write $\left\{A_{n}\right\}_{n} \sim_{\lambda} f$, if

$$
\lim _{n \rightarrow \infty} \frac{1}{d_{n}} \sum_{i=1}^{d_{n}} F\left(\lambda_{i}\left(A_{n}\right)\right)=\frac{1}{\mu_{k}(D)} \int_{D} \frac{\sum_{i=1}^{r} F\left(\lambda_{i}(f(\mathbf{x}))\right)}{r} \mathrm{~d} \mathbf{x}, \quad \forall F \in C_{c}(\mathbb{C}) .
$$

In this case, $f$ is called the spectral (or eigenvalue) symbol of $\left\{A_{n}\right\}_{n}$.

When we write a relation such as $\left\{A_{n}\right\}_{n} \sim_{\sigma} f$ or $\left\{A_{n}\right\}_{n} \sim_{\lambda} f$, it is understood that $\left\{A_{n}\right\}_{n}$ is a sequence of matrices and $f$ is a measurable function defined on a subset $D$ of some $\mathbb{R}^{k}$ with $0<\mu_{k}(D)<\infty$ and taking values in $\mathbb{C}^{r \times r}$ for some $r \geq 1$. In what follows, "iff" is an abbreviation of "if and only if".

S1. If $\left\{A_{n}\right\}_{n} \sim_{\sigma} f$, then $\left\{A_{n}\right\}_{n}$ is s.u.

S 2. If $\left\{A_{n}\right\}_{n} \sim_{\sigma} f$, then $\left\{A_{n}\right\}_{n}$ is s.v. iff $f$ is invertible a.e.

S3. If $\left\{A_{n}\right\}_{n} \sim_{\lambda} f$ and $\Lambda\left(A_{n}\right) \subseteq S$ for all $n$, then $\Lambda(f) \subseteq \bar{S}$ a.e.

S 4. If $A_{n}=X_{n}+Y_{n} \in \mathbb{C}^{d_{n} \times d_{n}}$ and

- each $X_{n}$ is Hermitian and $\left\{X_{n}\right\}_{n} \sim_{\lambda} f$,

- $\lim _{n \rightarrow \infty}\left(d_{n}\right)^{-1 / 2}\left\|Y_{n}\right\|_{2}=0$,

then $\left\{A_{n}\right\}_{n} \sim_{\lambda} f$.

S 5. Let $X_{n} \in \mathbb{C}^{d_{n} \times d_{n}}$ and $P_{n} \in \mathbb{C}^{d_{n} \times \delta_{n}}$, where $P_{n}^{*} P_{n}=I_{\delta_{n}}, \delta_{n} \leq d_{n}, \delta_{n} / d_{n} \rightarrow 1$.

- $\left\{X_{n}\right\}_{n} \sim_{\sigma} f$ iff $\left\{P_{n}^{*} X_{n} P_{n}\right\}_{n} \sim_{\sigma} f$.

- If the matrices $X_{n}$ are Hermitian, then $\left\{X_{n}\right\}_{n} \sim_{\lambda} f$ iff $\left\{P_{n}^{*} X_{n} P_{n}\right\}_{n} \sim_{\lambda} f$. 
Informal meaning. Assuming that $f: D \subset \mathbb{R}^{k} \rightarrow \mathbb{C}^{r \times r}$ possesses $r$ a.e. continuous eigenvalue functions $\lambda_{i}(f(\mathbf{x})), i=1, \ldots, r$, then the spectral distribution $\left\{A_{n}\right\}_{n} \sim_{\lambda} f$ has the following informal meaning: all the eigenvalues of $A_{n}$, except possibly for $o\left(d_{n}\right)$ outliers (with $d_{n}$ being the size of $A_{n}$ ), can be subdivided into $r$ different subsets of approximately the same cardinality and the eigenvalues belonging to the $i$ th subset are approximately equal to the samples of the $i$ th eigenvalue function $\lambda_{i}(f(\mathbf{x}))$ over a uniform grid in the domain $D$ (for $n$ large enough). For instance, if $k=1, d_{n}=n r$, and $D=[a, b]$, then, assuming we have no outliers, the eigenvalues of $A_{n}$ are approximately equal to

$$
\lambda_{i}\left(f\left(a+j \frac{b-a}{n}\right)\right), \quad j=1, \ldots, n, \quad i=1, \ldots, r,
$$

for $n$ large enough. Similarly, if $k=2, d_{n}=n^{2} r$, and $D=\left[a_{1}, b_{1}\right] \times\left[a_{2}, b_{2}\right]$, then, assuming we have no outliers, the eigenvalues of $A_{n}$ are approximately equal to

$$
\lambda_{i}\left(f\left(a_{1}+j_{1} \frac{b_{1}-a_{1}}{n}, a_{2}+j_{2} \frac{b_{2}-a_{2}}{n}\right)\right), \quad j_{1}, j_{2}=1, \ldots, n, \quad i=1, \ldots, r,
$$

for $n$ large enough. A completely analogous meaning can also be given for the singular value distribution $\left\{A_{n}\right\}_{n} \sim_{\sigma} f$.

Rearrangement. Assuming that $D=\left[a_{1}, b_{1}\right] \times \cdots \times\left[a_{k}, b_{k}\right]$ is an hyperrectangle in $\mathbb{R}^{k}$ and $f: D \rightarrow \mathbb{C}^{r \times r}$ is a measurable function possessing $r$ real-valued a.e. continuous eigenvalue functions $\lambda_{i}(f(\mathbf{x})), i=1, \ldots, r$, compute, for each $\rho \in \mathbb{N}$, the uniform samples

$$
\lambda_{i}\left(f\left(a_{1}+j_{1} \frac{b_{1}-a_{1}}{\rho}, \ldots, a_{k}+j_{k} \frac{b_{k}-a_{k}}{\rho}\right)\right), \quad j_{1}, \ldots, j_{k}=1, \ldots, \rho, \quad i=1, \ldots, r,
$$

sort them in non-decreasing order, and put them into a vector $\left(\varsigma_{1}, \varsigma_{2}, \ldots, \varsigma_{r \rho^{k}}\right)$. Let $\phi_{\rho}:[0,1] \rightarrow \mathbb{R}$ be the piecewise linear non-decreasing function that interpolates the samples $\left(\varsigma_{0}=\varsigma_{1}, \varsigma_{1}, \varsigma_{2}, \ldots, \varsigma_{r \rho^{k}}\right)$ over the nodes $\left(0, \frac{1}{r \rho^{k}}, \frac{2}{r \rho^{k}}, \ldots, 1\right)$, i.e.,

$$
\begin{cases}\phi_{\rho}\left(\frac{i}{r \rho^{k}}\right)=\varsigma_{i}, & i=0, \ldots, r \rho^{k}, \\ \phi_{\rho} \text { linear on }\left[\frac{i}{r \rho^{k}}, \frac{i+1}{r \rho^{k}}\right], & i=0, \ldots, r \rho^{k}-1 .\end{cases}
$$

When $\rho \rightarrow \infty$, the function $\phi_{\rho}$ converges a.e. to a function $\phi$, which is non-decreasing on $(0,1)$ and satisfies

$$
\int_{0}^{1} F(\phi(t)) \mathrm{d} t=\frac{1}{\mu_{k}(D)} \int_{D} \frac{\sum_{i=1}^{r} F\left(\lambda_{i}(f(\mathbf{x}))\right)}{r} \mathrm{~d} \mathbf{x}, \quad \forall F \in C_{c}(\mathbb{C}) .
$$

The function $\phi$ is referred to as the canonical rearranged version of $f$. What is interesting about $\phi$ is that if $\left\{A_{n}\right\}_{n} \sim_{\lambda} f$, then $\left\{A_{n}\right\}_{n} \sim_{\lambda} \phi$. In particular, if we have $\left\{A_{n}\right\}_{n} \sim_{\lambda} f$ (and hence also $\left.\left\{A_{n}\right\}_{n} \sim_{\lambda} \phi\right)$, then, for $n$ large enough, the eigenvalues of $A_{n}$, with the possible exception of $o\left(d_{n}\right)$ outliers $\left(d_{n}=\operatorname{size}\left(A_{n}\right)\right)$, are approximately equal to the samples of $\phi$ over a uniform grid in $[0,1]$.

Clustering and attraction. In what follows, if $f: D \subseteq \mathbb{R}^{k} \rightarrow \mathbb{C}^{r \times r}$ is a measurable matrix-valued function, its essential range is denoted by $\mathcal{E} \mathcal{R}(f)$ and is defined as

$$
\mathcal{E R}(f)=\left\{z \in \mathbb{C}: \mu_{k}\left\{\exists j \in\{1, \ldots, r\}: \lambda_{j}(f) \in D(z, \varepsilon)\right\}>0 \text { for all } \varepsilon>0\right\} .
$$




\section{ETNA}

Kent State University and

Johann Radon Institute (RICAM)

- Let $\left\{A_{n}\right\}_{n}$ be a sequence of matrices with $A_{n}$ of size $d_{n}$, and let $S$ be a nonempty subset of $\mathbb{C}$. We say that $\left\{A_{n}\right\}_{n}$ is weakly clustered at $S$ if

$$
\lim _{n \rightarrow \infty} \frac{\#\left\{j \in\left\{1, \ldots, d_{n}\right\}: \lambda_{j}\left(A_{n}\right) \notin D(S, \varepsilon)\right\}}{d_{n}}=0, \quad \forall \varepsilon>0 .
$$

- Let $\left\{A_{n}\right\}_{n}$ be a sequence of matrices with $A_{n}$ of size $d_{n}$, and let $z \in \mathbb{C}$. We say that $z$ strongly attracts the spectrum $\Lambda\left(A_{n}\right)$ with infinite order if, once we have ordered the eigenvalues of $A_{n}$ according to their distance from $z$,

$$
\left|\lambda_{1}\left(A_{n}\right)-z\right| \leq\left|\lambda_{2}\left(A_{n}\right)-z\right| \leq \ldots \leq\left|\lambda_{d_{n}}\left(A_{n}\right)-z\right|,
$$

the following limit relation holds for each fixed $j \geq 1$ :

$$
\lim _{n \rightarrow \infty}\left|\lambda_{j}\left(A_{n}\right)-z\right|=0 .
$$

CA 1. If $\left\{A_{n}\right\}_{n} \sim_{\lambda} f$, then $\left\{A_{n}\right\}_{n}$ is weakly clustered at $\mathcal{E R}(f)$ and each $z \in \mathcal{E} \mathcal{R}(f)$ strongly attracts $\Lambda\left(A_{n}\right)$ with infinite order.

Zero-distributed sequences. A sequence of matrices $\left\{Z_{n}\right\}_{n}$ such that $\left\{Z_{n}\right\}_{n} \sim_{\sigma} 0$ is referred to as a zero-distributed sequence. In other words, $\left\{Z_{n}\right\}_{n}$ is zero-distributed iff

$$
\lim _{n \rightarrow \infty} \frac{1}{d_{n}} \sum_{i=1}^{d_{n}} F\left(\sigma_{i}\left(Z_{n}\right)\right)=F(0), \quad \forall F \in C_{c}(\mathbb{R}),
$$

where $d_{n}$ is the size of $Z_{n}$. Given a sequence of matrices $\left\{Z_{n}\right\}_{n}$ with $Z_{n}$ of size $d_{n}$, the following properties hold. In what follows, we use the natural convention $C / \infty=0$ for all numbers $C$.

Z 1. $\left\{Z_{n}\right\}_{n} \sim_{\sigma} 0$ iff $Z_{n}=R_{n}+N_{n}$ with $\lim _{n \rightarrow \infty}\left(d_{n}\right)^{-1} \operatorname{rank}\left(R_{n}\right)=\lim _{n \rightarrow \infty}\left\|N_{n}\right\|=0$.

Z2. $\left\{Z_{n}\right\}_{n} \sim_{\sigma} 0$ if there exists a $p \in[1, \infty]$ such that $\lim _{n \rightarrow \infty}\left(d_{n}\right)^{-1 / p}\left\|Z_{n}\right\|_{p}=0$.

Sequences of block diagonal sampling matrices. If $n \in \mathbb{N}$ and $a:[0,1] \rightarrow \mathbb{C}^{s \times s}$, then the $n$th block (or $s$-block) diagonal sampling matrix generated by $a$ is the $s n \times s n$ block diagonal matrix given by

$$
D_{n}(a)=\underset{i=1, \ldots, n}{\operatorname{diag}} a\left(\frac{i}{n}\right) .
$$

$\left\{D_{n}(a)\right\}_{n}$ is the sequence of block (or $s$-block) diagonal sampling matrices generated by $a$. In what follows, we denote by $E^{[0,1]}$ the space of all functions from $[0,1]$ to the set $E$.

D 1. For every $n \in \mathbb{N}$ the map $D_{n}(\cdot):\left(\mathbb{C}^{s \times s}\right)^{[0,1]} \rightarrow \mathbb{C}^{s n \times s n}$

- is linear: $D_{n}(\alpha a+\beta b)=\alpha D_{n}(a)+\beta D_{n}(b)$;

- satisfies $D_{n}(a)^{*}=D_{n}\left(a^{*}\right)$.

D2. If $a_{i j}:[0,1] \rightarrow \mathbb{C}^{r \times r}$, for $i, j=1, \ldots, s$, and we define $a=\left[a_{i j}\right]_{i, j=1}^{s}$ and $D_{n}=\left[D_{n}\left(a_{i j}\right)\right]_{i, j=1}^{s}$, then $\Pi_{n, s, r} D_{n} \Pi_{n, s, r}^{T}=D_{n}(a)$.

Block Toeplitz sequences. If $n \in \mathbb{N}$ and $f:[-\pi, \pi] \rightarrow \mathbb{C}^{s \times s}$ is a function in $L^{1}([-\pi, \pi], s)$, then the $n$th ( $s$-block) Toeplitz matrix generated by $f$ is the $s n \times s n$ matrix given by

$$
T_{n}(f)=\left[f_{i-j}\right]_{i, j=1}^{n},
$$

where the $s \times s$ blocks $f_{k}$ are the Fourier coefficients of $f$,

$$
f_{k}=\frac{1}{2 \pi} \int_{-\pi}^{\pi} f(\theta) e^{-\mathrm{i} k \theta} \mathrm{d} \theta \in \mathbb{C}^{s \times s}, \quad k \in \mathbb{Z},
$$


and the integrals in the previous formula are computed componentwise. $\left\{T_{n}(f)\right\}_{n}$ is the (sblock) Toeplitz sequence generated by $f$. In what follows, for any function $f$ in $L^{p}([-\pi, \pi], s)$, we define

$$
\|f\|_{L^{p}}= \begin{cases}\left(\int_{D}\|f(\mathbf{x})\|_{p}^{p} \mathrm{~d} \mathbf{x}\right)^{1 / p}, & \text { if } 1 \leq p<\infty \\ {\operatorname{ess} \sup _{\mathbf{x} \in D}\|f(\mathbf{x})\|,}^{\text {if } p=\infty}\end{cases}
$$

T1. For every $n \in \mathbb{N}$ the map $T_{n}(\cdot): L^{1}([-\pi, \pi], s) \rightarrow \mathbb{C}^{s n \times s n}$

- is linear: $T_{n}(\alpha f+\beta g)=\alpha T_{n}(f)+\beta T_{n}(g)$;

- satisfies $T_{n}(f)^{*}=T_{n}\left(f^{*}\right)$.

T 2. If $f$ is Hermitian a.e., then $T_{n}(f)$ is Hermitian for all $n \in \mathbb{N}$.

T3. If $1 \leq p \leq \infty$ and $f \in L^{p}([-\pi, \pi], s)$, then $\left\|T_{n}(f)\right\|_{p} \leq(n / 2 \pi)^{1 / p}\|f\|_{L^{p}}$.

T4. If $f \in L^{1}([-\pi, \pi], s)$, then $\left\{T_{n}(f)\right\}_{n} \sim_{\sigma} f$. If in addition $f$ is Hermitian a.e., then $\left\{T_{n}(f)\right\}_{n} \sim_{\lambda} f$.

T 5. If $f_{1}, \ldots, f_{q} \in L^{\infty}([-\pi, \pi], s)$, then $n^{-1}\left\|\prod_{i=1}^{q} T_{n}\left(f_{i}\right)-T_{n}\left(\prod_{i=1}^{q} f_{i}\right)\right\|_{1} \rightarrow 0$.

T6. If $f_{i j} \in L^{1}([-\pi, \pi], r)$, for $i, j=1, \ldots, s$, and we set $f=\left[f_{i j}\right]_{i, j=1}^{s}$ and $T_{n}=\left[T_{n}\left(f_{i j}\right)\right]_{i, j=1}^{s}$, then $\Pi_{n, s, r} T_{n} \Pi_{n, s, r}^{T}=T_{n}(f)$.

Approximating classes of sequences. Let $\left\{A_{n}\right\}_{n}$ be a sequence of matrices and $\left\{\left\{B_{n, m}\right\}_{n}\right\}_{m}$ be a sequence of sequences of matrices with $A_{n}$ and $B_{n, m}$ of size $d_{n}$. We say that $\left\{\left\{B_{n, m}\right\}_{n}\right\}_{m}$ is an approximating class of sequences (a.c.s.) for $\left\{A_{n}\right\}_{n}$ if the following condition is met: for every $m$ there exists $n_{m}$ such that, for $n \geq n_{m}$,

$$
A_{n}=B_{n, m}+R_{n, m}+N_{n, m}, \quad \operatorname{rank}\left(R_{n, m}\right) \leq c(m) d_{n}, \quad\left\|N_{n, m}\right\| \leq \omega(m),
$$

where $n_{m}, c(m), \omega(m)$ depend only on $m$, and

$$
\lim _{m \rightarrow \infty} c(m)=\lim _{m \rightarrow \infty} \omega(m)=0 .
$$

We use the abbreviation "a.c.s." for both the singular "approximating class of sequences" and the plural "approximating classes of sequences". It turns out that, for each fixed sequence of positive integers $d_{n}$ such that $d_{n} \rightarrow \infty$, the notion of a.c.s. is a notion of convergence in the space $\mathscr{E}=\left\{\left\{A_{n}\right\}_{n}: A_{n} \in \mathbb{C}^{d_{n} \times d_{n}}\right.$ for every $\left.n\right\}$. More precisely, for every gauge function $\varphi$ and every $A \in \mathbb{C}^{\ell \times \ell}$, let

$$
p^{\varphi}(A)=\frac{1}{\ell} \sum_{i=1}^{\ell} \varphi\left(\sigma_{i}(A)\right)
$$

and define

$$
\begin{aligned}
p_{\text {a.c.s. }}^{\varphi}\left(\left\{A_{n}\right\}_{n}\right) & =\limsup _{n \rightarrow \infty} p\left(A_{n}\right), & & \left\{A_{n}\right\}_{n} \in \mathscr{E}, \\
d_{\text {a.c.s. }}^{\varphi}\left(\left\{A_{n}\right\}_{n},\left\{B_{n}\right\}_{n}\right) & =p_{\text {a.c.s. }}^{\varphi}\left(\left\{A_{n}-B_{n}\right\}_{n}\right), & & \left\{A_{n}\right\}_{n},\left\{B_{n}\right\}_{n} \in \mathscr{E} .
\end{aligned}
$$

Then, $d_{\text {a.c.s. }}^{\varphi}$ is a distance on $\mathscr{E}$ such that $d_{\text {a.c.s. }}^{\varphi}\left(\left\{A_{n}\right\}_{n},\left\{B_{n}\right\}_{n}\right)=0$ iff $\left\{A_{n}-B_{n}\right\}_{n}$ is zerodistributed. Moreover, $d_{\text {a.c.s. }}^{\varphi}$ turns $\mathscr{E}$ into a complete pseudometric space $\left(\mathscr{E}, d_{\text {a.c.s. }}^{\varphi}\right)$, where the statement " $\left\{\left\{B_{n, m}\right\}_{n}\right\}_{m}$ converges to $\left\{A_{n}\right\}_{n}$ " is equivalent to " $\left\{\left\{B_{n, m}\right\}_{n}\right\}_{m}$ is an a.c.s. for $\left\{A_{n}\right\}_{n}$ ". In particular, we can reformulate the definition of a.c.s. in the following way: $a$ sequence of sequences of matrices $\left\{\left\{B_{n, m}\right\}_{n}\right\}_{m}$ is said to be an a.c.s. for $\left\{A_{n}\right\}_{n}$ if $\left\{B_{n, m}\right\}_{n}$ converges to $\left\{A_{n}\right\}_{n}$ in $\left(\mathscr{E}, d_{\text {a.c.s. }}^{\varphi}\right)$ as $m \rightarrow \infty$, i.e., if $d_{\text {a.c.s. }}^{\varphi}\left(\left\{B_{n, m}\right\}_{n},\left\{A_{n}\right\}_{n}\right) \rightarrow 0$ as $m \rightarrow \infty$. The theory of a.c.s. may then be interpreted as an approximation theory for sequences 
of matrices, and for this reason we will use the convergence notation $\left\{B_{n, m}\right\}_{n} \stackrel{\text { a.c.s. }}{\longrightarrow}\left\{A_{n}\right\}_{n}$ to indicate that $\left\{\left\{B_{n, m}\right\}_{n}\right\}_{m}$ is an a.c.s. for $\left\{A_{n}\right\}_{n}$. In view of what follows, let $D \subset \mathbb{R}^{k}$ be a measurable set such that $0<\mu_{k}(D)<\infty$ and, for every gauge function $\varphi$, define

$$
\begin{aligned}
p_{\text {measure }}^{\varphi}(f) & =\frac{1}{\mu_{k}(D)} \int_{D} \frac{\sum_{i=1}^{r} \varphi\left(\sigma_{i}(f(\mathbf{x}))\right)}{r} \mathrm{~d} \mathbf{x}, & & f \in \mathfrak{M}_{D}^{(r)}, \\
d_{\text {measure }}^{\varphi}(f, g) & =p_{\text {measure }}^{\varphi}(f-g), & & f, g \in \mathfrak{M}_{D}^{(r)} .
\end{aligned}
$$

Then, $d_{\text {measure }}^{\varphi}$ is a distance on $\mathfrak{M}_{D}^{(r)}$ such that $d_{\text {measure }}^{\varphi}(f, g)=0$ iff $f=g$ a.e. Moreover, $d_{\text {measure }}^{\varphi}$ turns $\mathfrak{M}_{D}^{(r)}$ into a complete pseudometric space $\left(\mathfrak{M}_{D}^{(r)}, d_{\text {measure }}^{\varphi}\right)$, where the statement " $f_{m}$ converges to $f$ " is equivalent to " $f_{m}$ converges to $f$ in measure".

ACS 1. If $\left\{A_{n}\right\}_{n} \sim_{\sigma} f$, then $p_{\text {a.c.s. }}^{\varphi}\left(\left\{A_{n}\right\}_{n}\right)=p_{\text {measure }}^{\varphi}(f)$ for all gauge functions $\varphi$.

ACS 2. $\left\{A_{n}\right\}_{n} \sim_{\sigma} f$ iff there exist sequences of matrices $\left\{B_{n, m}\right\}_{n} \sim_{\sigma} f_{m}$ such that $\left\{B_{n, m}\right\}_{n} \stackrel{\text { a.c.s. }}{\longrightarrow}\left\{A_{n}\right\}_{n}$ and $f_{m} \rightarrow f$ in measure.

ACS 3. Suppose each $A_{n}$ is Hermitian. Then, $\left\{A_{n}\right\}_{n} \sim_{\lambda} f$ iff there exist sequences of Hermitian matrices $\left\{B_{n, m}\right\}_{n} \sim_{\lambda} f_{m}$ such that $\left\{B_{n, m}\right\}_{n} \stackrel{\text { a.c.s. }}{\longrightarrow}\left\{A_{n}\right\}_{n}$ and $f_{m} \rightarrow f$ in measure.

ACS 4. If $\left\{B_{n, m}\right\}_{n} \stackrel{\text { a.c.s. }}{\longrightarrow}\left\{A_{n}\right\}_{n}$ and $\left\{B_{n, m}^{\prime}\right\}_{n} \stackrel{\text { a.c.s. }}{\longrightarrow}\left\{A_{n}^{\prime}\right\}_{n}$ with $A_{n}$ and $A_{n}^{\prime}$ of the same size $d_{n}$, then

- $\left\{B_{n, m}^{*}\right\}_{n} \stackrel{\text { a.c.s. }}{\longrightarrow}\left\{A_{n}^{*}\right\}_{n}$,

- $\left\{\alpha B_{n, m}+\beta B_{n, m}^{\prime}\right\}_{n} \stackrel{\text { a.c.s. }}{\longrightarrow}\left\{\alpha A_{n}+\beta A_{n}^{\prime}\right\}_{n}$ for all $\alpha, \beta \in \mathbb{C}$,

- $\left\{B_{n, m} B_{n, m}^{\prime}\right\}_{n} \stackrel{\text { a.c.s. }}{\longrightarrow}\left\{A_{n} A_{n}^{\prime}\right\}_{n}$ whenever $\left\{A_{n}\right\}_{n},\left\{A_{n}^{\prime}\right\}_{n}$ are s.u.,

- $\left\{B_{n, m} C_{n}\right\}_{n} \stackrel{\text { a.c.s. }}{\longrightarrow}\left\{A_{n} C_{n}\right\}_{n}$ whenever $\left\{C_{n}\right\}_{n}$ is s.u.

ACS 5. If $A_{n}=\left[A_{n, i j}\right]_{i, j=1}^{s}, B_{n}^{(m)}=\left[B_{n, i j}^{(m)}\right]_{i, j=1}^{s}$ and $\left\{B_{n, i j}^{(m)}\right\}_{n} \stackrel{\text { a.c.s. }}{\longrightarrow}\left\{A_{n, i j}^{(m)}\right\}_{n}$, for $i, j=$ $1, \ldots, s$, then $\left\{B_{n}^{(m)}\right\}_{n} \stackrel{\text { a.c.s. }}{\longrightarrow}\left\{A_{n}\right\}_{n}$.

ACS 6. Let $p \in[1, \infty]$ and assume for each $m$ there is $n_{m}$ such that, for $n \geq n_{m}$, $\left\|A_{n}-B_{n, m}\right\|_{p} \leq \varepsilon(m, n)\left(d_{n}\right)^{1 / p}$, where $\lim _{m \rightarrow \infty} \limsup _{n \rightarrow \infty} \varepsilon(m, n)=0$ and $d_{n}$ is the size of both $A_{n}$ and $B_{n, m}$. Then $\left\{B_{n, m}\right\}_{n} \stackrel{\text { a.c.s. }}{\longrightarrow}\left\{A_{n}\right\}_{n}$.

Block generalized locally Toeplitz sequences. An $s$-block generalized locally Toeplitz (GLT) sequence $\left\{A_{n}\right\}_{n}$ is a special $s$-block matrix-sequence equipped with a measurable function $\kappa:[0,1] \times[-\pi, \pi] \rightarrow \mathbb{C}^{s \times s}$, the so-called symbol (or kernel). Unless specified otherwise, the notation $\left\{A_{n}\right\}_{n} \sim_{\text {GLT }} \kappa$ means that $\left\{A_{n}\right\}_{n}$ is an $s$-block GLT sequence with symbol $\kappa \in \mathfrak{M}^{(s)}$. The symbol of an $s$-block GLT sequence is unique in the sense that if $\left\{A_{n}\right\}_{n} \sim_{\mathrm{GLT}} \kappa$ and $\left\{A_{n}\right\}_{n} \sim_{\mathrm{GLT}} \xi$, then $\kappa=\xi$ a.e. in $[0,1] \times[-\pi, \pi]$. Conversely, if $\left\{A_{n}\right\}_{n} \sim_{\mathrm{GLT}} \kappa$ and $\kappa=\xi$ a.e. in $[0,1] \times[-\pi, \pi]$, then $\left\{A_{n}\right\}_{n} \sim_{\mathrm{GLT}} \xi$. In addition, any measurable function $\kappa:[0,1] \times[-\pi, \pi] \rightarrow \mathbb{C}^{s \times s}$ is the symbol of some $s$-block GLT sequence $\left\{A_{n}\right\}_{n}$.

GLT 1. If $\left\{A_{n}\right\}_{n} \sim_{\mathrm{GLT}} \kappa$, then $\left\{A_{n}\right\}_{n} \sim_{\sigma} \kappa$. If $\left\{A_{n}\right\}_{n} \sim_{\mathrm{GLT}} \kappa$ and the matrices $A_{n}$ are Hermitian, then $\kappa$ is Hermitian a.e. and $\left\{A_{n}\right\}_{n} \sim_{\lambda} \kappa$.

GLT 2. If $\left\{A_{n}\right\}_{n} \sim_{\mathrm{GLT}} \kappa$ with $A_{n}=X_{n}+Y_{n}$, and

- every $X_{n}$ is Hermitian,

- $n^{-1 / 2}\left\|Y_{n}\right\|_{2} \rightarrow 0$,

then $\left\{P_{n}^{*} A_{n} P_{n}\right\}_{n} \sim_{\sigma, \lambda} \kappa$ for every sequence $\left\{P_{n}\right\}_{n}$ such that $P_{n} \in \mathbb{C}^{s n \times \delta_{n}}$, $P_{n}^{*} P_{n}=I_{\delta_{n}}, \delta_{n} \leq s n$, and $\delta_{n} / s n \rightarrow 1$.

GLT 3. We have

- $\left\{T_{n}(f)\right\}_{n} \sim_{\mathrm{GLT}} \kappa(x, \theta)=f(\theta)$ if $f \in L^{1}([-\pi, \pi], s)$,

- $\left\{D_{n}(a)\right\}_{n} \sim_{\mathrm{GLT}} \kappa(x, \theta)=a(x)$ if $a:[0,1] \rightarrow \mathbb{C}^{s \times s}$ is continuous a.e., 
- $\left\{Z_{n}\right\}_{n} \sim_{\mathrm{GLT}} \kappa(x, \theta)=O_{s}$ iff $\left\{Z_{n}\right\}_{n} \sim_{\sigma} 0$.

GLT 4. If $\left\{A_{n}\right\}_{n} \sim_{\mathrm{GLT}} \kappa$ and $\left\{B_{n}\right\}_{n} \sim_{\mathrm{GLT}} \xi$, then

- $\left\{A_{n}^{*}\right\}_{n} \sim_{\mathrm{GLT}} \kappa^{*}$,

- $\left\{\alpha A_{n}+\beta B_{n}\right\}_{n} \sim_{\mathrm{GLT}} \alpha \kappa+\beta \xi$ for all $\alpha, \beta \in \mathbb{C}$,

- $\left\{A_{n} B_{n}\right\}_{n} \sim_{\mathrm{GLT}} \kappa \xi$,

- $\left\{A_{n}^{\dagger}\right\}_{n} \sim_{\mathrm{GLT}} \kappa^{-1}$ if $\kappa$ is invertible a.e.

GLT 5. If $\left\{A_{n}\right\}_{n} \sim_{\mathrm{GLT}} \kappa$ and each $A_{n}$ is Hermitian, then $\left\{f\left(A_{n}\right)\right\}_{n} \sim_{\mathrm{GLT}} f(\kappa)$ for every continuous function $f: \mathbb{C} \rightarrow \mathbb{C}$.

GLT 6. If $\left\{A_{n, i j}\right\}_{n} \sim_{\mathrm{GLT}} \kappa_{i j} \in \mathfrak{M}^{(r)}$, for $i, j=1, \ldots, s$, and we set $\kappa=\left[\kappa_{i j}\right]_{i, j=1}^{s}$ and $A_{n}=\left[A_{n, i j}\right]_{i, j=1}^{s}$, then $\left\{\Pi_{n, s, r} A_{n} \Pi_{n, s, r}^{T}\right\}_{n} \sim_{\mathrm{GLT}} \kappa \in \mathfrak{M}^{(r s)}$.

GLT 7. $\left\{A_{n}\right\}_{n} \sim_{\mathrm{GLT}} \kappa$ iff there exist $s$-block GLT sequences $\left\{B_{n, m}\right\}_{n} \sim_{\mathrm{GLT}} \kappa_{m}$ such that $\left\{B_{n, m}\right\}_{n} \stackrel{\text { a.c.s. }}{\longrightarrow}\left\{A_{n}\right\}_{n}$ and $\kappa_{m} \rightarrow \kappa$ in measure.

GLT 8. If $\left\{A_{n}\right\}_{n} \sim_{\mathrm{GLT}} \kappa$ and $\left\{B_{n}\right\}_{n} \sim_{\mathrm{GLT}} \xi$, then, for every gauge function $\varphi$, we have $d_{\text {a.c.s. }}^{\varphi}\left(\left\{A_{n}\right\}_{n},\left\{B_{n}\right\}_{n}\right)=d_{\text {measure }}^{\varphi}(\kappa, \xi)$.

GLT 9. If $\left\{A_{n}\right\}_{n} \sim_{\mathrm{GLT}} \kappa$, then there exist functions $a_{i, m}, f_{i, m}, i=1, \ldots, N_{m}$, such that

- $a_{i, m}:[0,1] \rightarrow \mathbb{C}$ belongs to $C^{\infty}([0,1])$ and $f_{i, m}$ is a trigonometric monomial in $\left\{e^{\mathrm{i} j \theta} E_{\alpha \beta}^{(s)}: j \in \mathbb{Z}, 1 \leq \alpha, \beta \leq s\right\}$,

- $\sum_{i=1}^{N_{m}} a_{i, m}(x) f_{i, m}(\theta) \rightarrow \kappa(x, \theta)$ a.e.,

- $\left\{\sum_{i=1}^{N_{m}} D_{n}\left(a_{i, m} I_{s}\right) T_{n}\left(f_{i, m}\right)\right\}_{n} \stackrel{\text { a.c.s. }}{\longrightarrow}\left\{A_{n}\right\}_{n}$.

6. Applications. In this chapter we present several emblematic applications of the theory of block GLT sequences to the computation of the singular value and eigenvalue distribution of sequences of matrices arising from the numerical discretization of DEs. In order to understand the content of this chapter, it is enough that the reader knows the summary of Chapter 5 and possesses the necessary prerequisites, most of which have been addressed in Chapter 2. Indeed, our derivations here will never refer to Chapters $1-4$, i.e., they will only rely on the summary of Chapter 5.

6.1. FD discretization of systems of DEs. Consider the following system of DEs:

$$
\left\{\begin{array}{cl}
-a_{11}(x) u_{1}^{\prime \prime}(x)+a_{12}(x) u_{2}^{\prime}(x)=f_{1}(x), \quad & x \in(0,1), \\
a_{21}(x) u_{1}^{\prime}(x)+a_{22}(x) u_{2}(x)=f_{2}(x), \quad x \in(0,1), \\
u_{1}(0)=0, \quad u_{1}(1)=0, \\
u_{2}(0)=0, \quad u_{2}(1)=0 .
\end{array}\right.
$$

In this section we consider the classical central FD discretization of (6.1). Through the theory of block GLT sequences we show that, under suitable assumptions on the DE coefficients $a_{11}, a_{12}, a_{21}, a_{22}$, the corresponding sequence of (normalized) FD discretization matrices enjoys a spectral distribution described by a $2 \times 2$ matrix-valued function. We remark that the number 2 , which identifies the matrix space $\mathbb{C}^{2 \times 2}$ where the spectral symbol takes values, coincides with the number of equations that compose the system (6.1). In what follows, we use the notation

$$
\underset{j=1, \ldots, n}{\operatorname{tridiag}}\left[\beta_{j}\left|\alpha_{j}\right| \gamma_{j}\right]=\left[\begin{array}{ccccc}
\alpha_{1} & \gamma_{1} & & & \\
\beta_{2} & \alpha_{2} & \gamma_{2} & & \\
& \ddots & \ddots & \ddots & \\
& & \beta_{n-1} & \alpha_{n-1} & \gamma_{n-1} \\
& & & \beta_{n} & \alpha_{n}
\end{array}\right]
$$


FD discretization. Let $n \geq 1$, set $h=\frac{1}{n+1}$ and $x_{j}=j h$, for $j=0, \ldots, n+1$. Using the classical central FD schemes $(-1,2,-1)$ and $\frac{1}{2}(-1,0,1)$ for the discretization of, respectively, the (negative) second derivative and the first derivative, for each $j=1, \ldots, n$, we obtain the following approximations:

$$
\begin{aligned}
{\left.\left[-a_{11}(x) u_{1}^{\prime \prime}(x)+a_{12}(x) u_{2}^{\prime}(x)\right]\right|_{x=x_{j}} \approx } & a_{11}\left(x_{j}\right) \frac{-u_{1}\left(x_{j+1}\right)+2 u_{1}\left(x_{j}\right)-u_{1}\left(x_{j-1}\right)}{h^{2}} \\
& +a_{12}\left(x_{j}\right) \frac{u_{2}\left(x_{j+1}\right)-u_{2}\left(x_{j-1}\right)}{2 h}, \\
{\left.\left[a_{21}(x) u_{1}^{\prime}(x)+a_{22}(x) u_{2}(x)\right]\right|_{x=x_{j}} \approx } & a_{21}\left(x_{j}\right) \frac{u_{1}\left(x_{j+1}\right)-u_{1}\left(x_{j-1}\right)}{2 h}+a_{22}\left(x_{j}\right) u_{2}\left(x_{j}\right) .
\end{aligned}
$$

This means that the nodal values of the solutions $u_{1}, u_{2}$ of (6.1) satisfy approximately the equations

$$
\begin{aligned}
& a_{11}\left(x_{j}\right)\left[-u_{1}\left(x_{j+1}\right)+2 u_{1}\left(x_{j}\right)-u_{1}\left(x_{j-1}\right)\right]+\frac{h}{2} a_{12}\left(x_{j}\right)\left[u_{2}\left(x_{j+1}\right)-u_{2}\left(x_{j-1}\right)\right] \\
& \quad=h^{2} f_{1}\left(x_{j}\right) \\
& \frac{1}{2} a_{21}\left(x_{j}\right)\left[u_{1}\left(x_{j+1}\right)-u_{1}\left(x_{j-1}\right)\right]+h a_{22}\left(x_{j}\right) u_{2}\left(x_{j}\right) \\
& \quad=h f_{2}\left(x_{j}\right)
\end{aligned}
$$

for $j=1, \ldots, n$. We then approximate the solution $u_{1}$ (respectively, $u_{2}$ ) by the piecewise linear function that takes the value $u_{1, j}$ (respectively, $u_{2, j}$ ) at $x_{j}$ for all $j=0, \ldots, n+1$, where $u_{1,0}=u_{1, n+1}=u_{2,0}=u_{2, n+1}=0$ and the vectors $\mathbf{u}_{1}=\left(u_{1,1}, \ldots, u_{1, n}\right)^{T}$ and $\mathbf{u}_{2}=\left(u_{2,1}, \ldots, u_{2, n}\right)^{T}$ solve the linear system

$$
\begin{aligned}
a_{11}\left(x_{j}\right)\left[-u_{1, j+1}+2 u_{1, j}-u_{1, j-1}\right]+\frac{h}{2} a_{12}\left(x_{j}\right)\left[u_{2, j+1}-u_{2, j-1}\right] & =h^{2} f_{1}\left(x_{j}\right), \\
\frac{1}{2} a_{21}\left(x_{j}\right)\left[u_{1, j+1}-u_{1, j-1}\right]+h a_{22}\left(x_{j}\right) u_{2, j} & =h f_{2}\left(x_{j}\right),
\end{aligned}
$$

for $j=1, \ldots, n$. This linear system can be rewritten in matrix form as follows:

$$
A_{n}\left[\begin{array}{l}
\mathbf{u}_{1} \\
\mathbf{u}_{2}
\end{array}\right]=\left[\begin{array}{c}
h^{2} \mathbf{f}_{1} \\
h \mathbf{f}_{2}
\end{array}\right]
$$

where $\mathbf{f}_{1}=\left[f_{1}\left(x_{j}\right)\right]_{j=1}^{n}, \mathbf{f}_{2}=\left[f_{2}\left(x_{j}\right)\right]_{j=1}^{n}$,

$$
A_{n}=\left[\begin{array}{ll}
K_{n}\left(a_{11}\right) & h H_{n}\left(a_{12}\right) \\
H_{n}\left(a_{21}\right) & h M_{n}\left(a_{22}\right)
\end{array}\right]=\left[\begin{array}{ll}
K_{n}\left(a_{11}\right) & H_{n}\left(a_{12}\right) \\
H_{n}\left(a_{21}\right) & M_{n}\left(a_{22}\right)
\end{array}\right]\left[\begin{array}{ll}
I_{n} & O_{n} \\
O_{n} & h I_{n}
\end{array}\right]
$$

and

$$
\begin{aligned}
K_{n}\left(a_{11}\right) & =\underset{j=1, \ldots, n}{\operatorname{tridiag}}\left[-a_{11}\left(x_{j}\right)\left|2 a_{11}\left(x_{j}\right)\right|-a_{11}\left(x_{j}\right)\right] \\
& =\left(\underset{j=1, \ldots, n}{\operatorname{diag}} a_{11}\left(x_{j}\right)\right) T_{n}(2-2 \cos \theta) \\
H_{n}\left(a_{12}\right) & =\underset{j=1, \ldots, n}{\operatorname{tridiag}}\left[-\frac{1}{2} a_{12}\left(x_{j}\right)|0| \frac{1}{2} a_{12}\left(x_{j}\right)\right] \\
& =\left(\operatorname{diag}_{j=1, \ldots, n} a_{12}\left(x_{j}\right)\right) T_{n}(-\mathrm{i} \sin \theta)
\end{aligned}
$$




$$
\begin{aligned}
H_{n}\left(a_{21}\right) & =\underset{j=1, \ldots, n}{\operatorname{tridiag}}\left[-\frac{1}{2} a_{21}\left(x_{j}\right)|0| \frac{1}{2} a_{21}\left(x_{j}\right)\right] \\
& =\left(\underset{j=1, \ldots, n}{\operatorname{diag}} a_{21}\left(x_{j}\right)\right) T_{n}(-\mathrm{i} \sin \theta), \\
M_{n}\left(a_{22}\right) & =\underset{j=1, \ldots, n}{\operatorname{diag}} a_{22}\left(x_{j}\right) .
\end{aligned}
$$

In view of (6.3), the linear system (6.2) is equivalent to

$$
B_{n}\left[\begin{array}{c}
\mathbf{v}_{1} \\
\mathbf{v}_{2}
\end{array}\right]=\left[\begin{array}{c}
h^{2} \mathbf{f}_{1} \\
h \mathbf{f}_{2}
\end{array}\right]
$$

where $\mathbf{v}_{1}=\mathbf{u}_{1}, \mathbf{v}_{2}=h \mathbf{u}_{2}$, and

$$
B_{n}=\left[\begin{array}{ll}
K_{n}\left(a_{11}\right) & H_{n}\left(a_{12}\right) \\
H_{n}\left(a_{21}\right) & M_{n}\left(a_{22}\right)
\end{array}\right] .
$$

GLT analysis of the FD discretization matrices. In the main result of this section (Theorem 6.1), we show that $\left\{\Pi_{n, 2} B_{n} \Pi_{n, 2}^{T}\right\}_{n}$ is a 2-block GLT sequence whose symbol $\kappa(x, \theta)$ is a $2 \times 2$ matrix-valued function obtained by replacing the GLT sequences $\left\{K_{n}\left(a_{11}\right)\right\}_{n}$, $\left\{H_{n}\left(a_{12}\right)\right\}_{n},\left\{H_{n}\left(a_{21}\right)\right\}_{n},\left\{M_{n}\left(a_{22}\right)\right\}_{n}$ appearing in (6.4) with the corresponding symbols $a_{11}(x)(2-2 \cos \theta),-\mathrm{i} a_{12}(x) \sin \theta,-\mathrm{i} a_{21}(x) \sin \theta, a_{22}(x)$. In this regard, note that, assuming for instance that $a_{11}, a_{12}, a_{21}, a_{22} \in C([0,1])$, we have

$$
\begin{aligned}
& \left\{K_{n}\left(a_{11}\right)\right\}_{n} \sim_{\mathrm{GLT}} a_{11}(x)(2-2 \cos \theta), \\
& \left\{H_{n}\left(a_{12}\right)\right\}_{n} \sim_{\mathrm{GLT}}-\mathrm{i} a_{12}(x) \sin \theta, \\
& \left\{H_{n}\left(a_{21}\right)\right\}_{n} \sim_{\mathrm{GLT}}-\mathrm{i} a_{21}(x) \sin \theta, \\
& \left\{M_{n}\left(a_{22}\right)\right\}_{n} \sim_{\mathrm{GLT}} a_{22}(x) .
\end{aligned}
$$

To prove (6.5), it suffices to observe that

$$
\begin{aligned}
& \left\|K_{n}\left(a_{11}\right)-D_{n}\left(a_{11}\right) T_{n}(2-2 \cos \theta)\right\| \\
& \leq\left\|\operatorname{diag}_{j=1, \ldots, n} a_{11}\left(x_{j}\right)-D_{n}\left(a_{11}\right)\right\|\left\|T_{n}(2-2 \cos \theta)\right\| \\
& =\max _{j=1, \ldots, n}\left|a_{11}\left(x_{j}\right)-a_{11}\left(\frac{j}{n}\right)\right|\left\|T_{n}(2-2 \cos \theta)\right\| \leq 4 \omega_{a_{11}}(h),
\end{aligned}
$$

where $\omega_{a_{11}}(\cdot)$ is the modulus of continuity of $a_{11}$. Since $\omega_{a_{11}}(h) \rightarrow 0$ as $n \rightarrow \infty$, we have $\left\{K_{n}\left(a_{11}\right)-D_{n}\left(a_{11}\right) T_{n}(2-2 \cos \theta)\right\}_{n} \sim_{\sigma} 0$ by Z 1, and so GLT 3 and GLT 4 immediately yield (6.5). The relations (6.6)-(6.8) are proved in the same way.

THEOREM 6.1. Suppose that $a_{11}, a_{12}, a_{21}, a_{22} \in C([0,1])$, and set $C_{n}=\Pi_{n, 2} B_{n} \Pi_{n, 2}^{T}$. Then

$$
\left\{C_{n}\right\}_{n} \sim_{\mathrm{GLT}} \kappa(x, \theta)=\left[\begin{array}{cc}
a_{11}(x)(2-2 \cos \theta) & -\mathrm{i} a_{12}(x) \sin \theta \\
-\mathrm{i} a_{21}(x) \sin \theta & a_{22}(x)
\end{array}\right]
$$

and we have

$$
\left\{C_{n}\right\}_{n} \sim_{\sigma} \kappa(x, \theta) .
$$

If moreover $a_{21}=-a_{12}$, then we also have

$$
\left\{C_{n}\right\}_{n} \sim_{\lambda} \kappa(x, \theta) .
$$




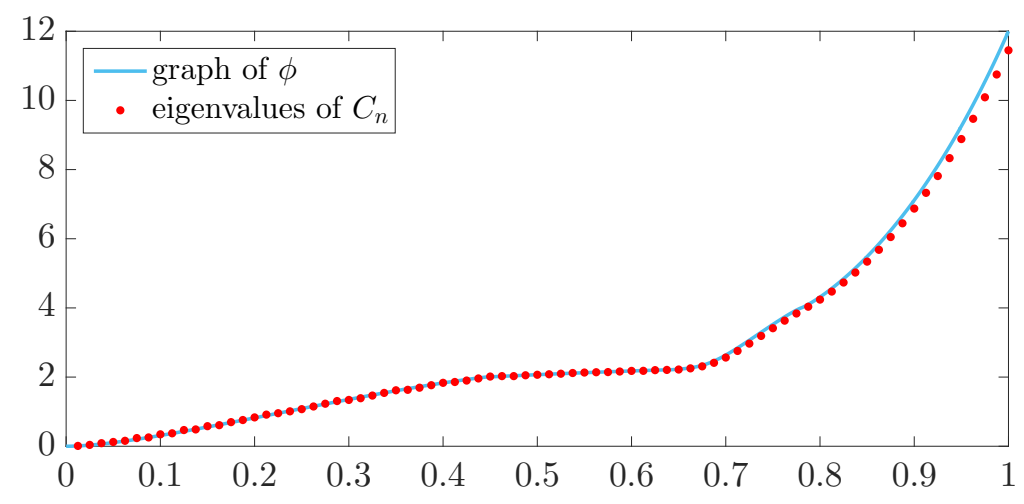

FIG. 6.1. Comparison between the spectrum of $C_{n}$ and the rearranged version $\phi$ of the symbol $\kappa(x, \theta)$ for $a_{11}(x)=2+\cos (\pi x), a_{12}(x)=-a_{21}(x)=e^{-x} \sin (\pi x), a_{22}(x)=2 x+\sin (\pi x)$, and $n=40$.

Proof. The GLT relation (6.9) follows immediately from (6.4)-(6.8) and GLT 6. The singular value distribution (6.10) follows from (6.9) and GLT 1. It only remains to prove (6.11) in the case where $a_{21}=-a_{12}$. In this case, we have

$$
B_{n}=\left[\begin{array}{cc}
K_{n}\left(a_{11}\right) & H_{n}\left(a_{12}\right) \\
-H_{n}\left(a_{12}\right) & M_{n}\left(a_{22}\right)
\end{array}\right] .
$$

Consider the symmetric approximation of $B_{n}$ given by

$$
\tilde{B}_{n}=\left[\begin{array}{cc}
\tilde{K}_{n}\left(a_{11}\right) & \tilde{H}_{n}\left(a_{12}\right) \\
-\tilde{H}_{n}\left(a_{12}\right) & M_{n}\left(a_{22}\right)
\end{array}\right],
$$

where

$$
\begin{aligned}
& \tilde{K}_{n}\left(a_{11}\right)=\underset{j=1, \ldots, n}{\operatorname{tridiag}}\left[-a_{11}\left(x_{j-1}\right)\left|2 a_{11}\left(x_{j}\right)\right|-a_{11}\left(x_{j}\right)\right], \\
& \tilde{H}_{n}\left(a_{12}\right)=\underset{j=1, \ldots, n}{\operatorname{tridiag}}\left[-\frac{1}{2} a_{12}\left(x_{j-1}\right)|0| \frac{1}{2} a_{12}\left(x_{j}\right)\right] .
\end{aligned}
$$

It is not difficult to see that $\left\|B_{n}-\tilde{B}_{n}\right\| \rightarrow 0$ as $n \rightarrow \infty$ by invoking the inequality $\mathbf{N} \mathbf{1}$. Thus, setting $\tilde{C}_{n}=\Pi_{n, 2} \tilde{B}_{n} \Pi_{n, 2}^{T}$, we have $\left\|C_{n}-\tilde{C}_{n}\right\|=\left\|B_{n}-\tilde{B}_{n}\right\| \rightarrow 0$ as $n \rightarrow \infty$. Therefore:

- in view of the decomposition $\tilde{C}_{n}=C_{n}+\left(\tilde{C}_{n}-C_{n}\right)$, we have $\left\{\tilde{C}_{n}\right\}_{n} \sim_{\mathrm{GLT}} \kappa(x, \theta)$ by (6.9), Z 1, GLT 3, and GLT 4, so in particular, $\left\{\tilde{C}_{n}\right\}_{n} \sim_{\lambda} \kappa(x, \theta)$ by GLT 1 because $\tilde{C}_{n}$ is symmetric;

- $\left\|C_{n}-\tilde{C}_{n}\right\|_{2} \leq \sqrt{2 n}\left\|C_{n}-\tilde{C}_{n}\right\|=o(\sqrt{n})$ as $n \rightarrow \infty$.

Thus, (6.11) follows from GLT 2.

EXAMPLE 6.2. Suppose that $a_{11}, a_{12}, a_{21}, a_{22} \in C([0,1])$ and $a_{21}=-a_{12}$, so that $\left\{C_{n}\right\}_{n} \sim_{\lambda} \kappa(x, \theta)$ by Theorem 6.1. The eigenvalue functions of $\kappa(x, \theta)$ are given by

$$
\begin{aligned}
\lambda_{1,2}(\kappa(x, \theta))= & \frac{a_{11}(x)(2-2 \cos \theta)+a_{22}(x)}{2} \\
& \pm \frac{\sqrt{\left(a_{11}(x)(2-2 \cos \theta)-a_{22}(x)\right)^{2}+4\left(a_{12}(x) \sin \theta\right)^{2}}}{2}
\end{aligned}
$$

and are continuous on $[0,1] \times[-\pi, \pi]$. Let $\phi$ be the canonical rearranged version of $\kappa(x, \theta)$ obtained as the limit of the piecewise linear functions $\phi_{\rho}$ according to the construction in 
Chapter 5 (fifth paragraph). Figure 6.1 displays the graph of $\phi$ and the eigenvalues $\lambda_{1}, \ldots, \lambda_{2 n}$ of $C_{n}$ for $a_{11}(x)=2+\cos (\pi x), a_{12}(x)=-a_{21}(x)=e^{-x} \sin (\pi x), a_{22}(x)=2 x+\sin (\pi x)$, and $n=40$. The graph of $\phi$ has been obtained by plotting the graph of $\phi_{\rho}$ corresponding to a large value of $\rho$. The eigenvalues of $C_{n}$, which turn out to be real although $C_{n}$ is not symmetric, have been sorted in non-decreasing order and placed at the points $\left(t_{q}, \lambda_{q}\right)$ with $t_{q}=\frac{q}{2 n}, q=1, \ldots, 2 n$. We clearly see from the figure an excellent agreement between $\phi$ and the eigenvalues of $C_{n}$, as predicted in Chapter 5 (fifth paragraph). In particular, we observe no outliers in this case.

6.2. Higher-order FE discretization of diffusion equations. Consider the diffusion problem

$$
\left\{\begin{aligned}
-\left(a(x) u^{\prime}(x)\right)^{\prime} & =f(x), \quad x \in(0,1), \\
u(0)=u(1) & =0 .
\end{aligned}\right.
$$

In this section we consider the higher-order FE discretization of (6.12). Through the theory of block GLT sequences we show that the corresponding sequence of (normalized) FE discretization matrices enjoys a spectral distribution described by a $(p-k) \times(p-k)$ matrix-valued function, where $p$ and $k$ represent, respectively, the degree and the smoothness of the piecewise polynomial functions involved in the FE approximation. Note that this result represents a remarkable argument in support of [54, Conjecture 2].

FE discretization. The weak form of (6.12) reads as follows [25, Chapter 8]: find $u \in H_{0}^{1}([0,1])$ such that

$$
\int_{0}^{1} a(x) u^{\prime}(x) w^{\prime}(x) \mathrm{d} x=\int_{0}^{1} f(x) w(x) \mathrm{d} x, \quad \forall w \in H_{0}^{1}([0,1]) .
$$

In the FE method [66, Chapter 4], we fix a set of basis functions $\left\{\varphi_{1}, \ldots, \varphi_{N}\right\} \subset H_{0}^{1}([0,1])$, and we look for an approximation of the exact solution in the space $\mathcal{W}=\operatorname{span}\left(\varphi_{1}, \ldots, \varphi_{N}\right)$ by solving the following discrete problem: find $u_{\mathcal{W}} \in \mathcal{W}$ such that

$$
\int_{0}^{1} a(x) u_{\mathcal{W}}^{\prime}(x) w^{\prime}(x) \mathrm{d} x=\int_{0}^{1} f(x) w(x) \mathrm{d} x, \quad \forall w \in \mathcal{W} .
$$

Since $\left\{\varphi_{1}, \ldots, \varphi_{N}\right\}$ is a basis of $\mathcal{W}$, we can write $u_{\mathcal{W}}=\sum_{j=1}^{N} u_{j} \varphi_{j}$ for a unique vector $\mathbf{u}=\left(u_{1}, \ldots, u_{N}\right)^{T}$. By linearity, the computation of $u_{\mathcal{W}}$ (i.e., of $\mathbf{u}$ ) reduces to solving the linear system

$$
A \mathbf{u}=\mathbf{f},
$$

where $\mathbf{f}=\left(\int_{0}^{1} f(x) \varphi_{1}(x) \mathrm{d} x, \ldots, \int_{0}^{1} f(x) \varphi_{N}(x) \mathrm{d} x\right)^{T}$ and $A$ is the stiffness matrix,

$$
A=\left[\int_{0}^{1} a(x) \varphi_{j}^{\prime}(x) \varphi_{i}^{\prime}(x) \mathrm{d} x\right]_{i, j=1}^{N} .
$$

$p$-degree $C^{k} \mathrm{~B}$-spline basis functions. Following the higher-order $\mathrm{FE}$ approach, the basis functions $\varphi_{1}, \ldots, \varphi_{N}$ will be chosen as piecewise polynomials of degree $p \geq 1$. More precisely, for $p, n \geq 1$ and $0 \leq k \leq p-1$, let $B_{1,[p, k]}, \ldots, B_{n(p-k)+k+1,[p, k]}: \mathbb{R} \rightarrow \mathbb{R}$ be 
the B-splines of degree $p$ and smoothness $C^{k}$ defined on the knot sequence

$$
\begin{aligned}
& \left\{\tau_{1}, \ldots, \tau_{n(p-k)+p+k+2}\right\} \\
& \quad=\{\underbrace{0, \ldots, 0}_{p+1}, \underbrace{\frac{1}{n}, \ldots, \frac{1}{n}}_{p-k}, \underbrace{\frac{2}{n}, \ldots, \frac{2}{n}}_{p-k}, \ldots, \underbrace{\frac{n-1}{n}, \ldots, \frac{n-1}{n}}_{p-k}, \underbrace{1, \ldots, 1}_{p+1}\} .
\end{aligned}
$$

We collect here a few properties of $B_{1,[p, k]}, \ldots, B_{n(p-k)+k+1,[p, k]}$ that we shall need later on. For the formal definition of B-splines as well as for the proof of the properties listed below, see $[31,73]$.

- The support of the $i$ th B-spline is given by

$$
\operatorname{supp}\left(B_{i,[p, k]}\right)=\left[\tau_{i}, \tau_{i+p+1}\right], \quad i=1, \ldots, n(p-k)+k+1 .
$$

- Except for the first and the last one, all the other B-splines vanish on the boundary of $[0,1]$, i.e.,

$$
B_{i,[p, k]}(0)=B_{i,[p, k]}(1)=0, \quad i=2, \ldots, n(p-k)+k .
$$

- $\left\{B_{1,[p, k]}, \ldots, B_{n(p-k)+k+1,[p, k]}\right\}$ is a basis for the space of piecewise polynomial functions of degree $p$ and smoothness $C^{k}$, that is,

$$
\mathcal{V}_{n,[p, k]}=\left\{v \in C^{k}([0,1]):\left.v\right|_{\left[\frac{i}{n}, \frac{i+1}{n}\right]} \in \mathbb{P}_{p} \text { for } i=0, \ldots, n-1\right\},
$$

where $\mathbb{P}_{p}$ is the space of polynomials of degree less than or equal to $p$. Moreover, $\left\{B_{2,[p, k]}, \ldots, B_{n(p-k)+k,[p, k]}\right\}$ is a basis for the space

$$
\mathcal{W}_{n,[p, k]}=\left\{w \in \mathcal{V}_{n,[p, k]}: w(0)=w(1)=0\right\} .
$$

- The B-splines form a non-negative partition of unity over $[0,1]$ :

$$
B_{i,[p, k]} \geq 0 \text { over } \mathbb{R}, \quad i=1, \ldots, n(p-k)+k+1,
$$

$$
\sum_{i=1}^{n(p-k)+k+1} B_{i,[p, k]}=1 \text { over }[0,1] .
$$

- The derivatives of the B-splines satisfy

$$
\sum_{i=1}^{n(p-k)+k+1}\left|B_{i,[p, k]}^{\prime}\right| \leq C_{p} n \text { over } \mathbb{R}
$$

where $C_{p}$ is a constant depending only on $p$. Note that the derivatives $B_{i,[p, k]}^{\prime}$ may not be defined at some of the grid points $0, \frac{1}{n}, \frac{2}{n}, \ldots, \frac{n-1}{n}, 1$ in the case of $C^{0}$ smoothness $(k=0)$. In (6.18) it is assumed that the undefined values are excluded from the summation.

- For every $\mathbf{y}=\left(y_{1}, \ldots, y_{n(p-k)+k+1}\right) \in \mathbb{R}^{n(p-k)+k+1}$, we have

$$
\left\|\sum_{i=1}^{n(p-k)+k+1} y_{i} B_{i,[p, k]}\right\|_{L^{2}([0,1])}^{2}=\int_{0}^{1}\left(\sum_{i=1}^{n(p-k)+k+1} y_{i} B_{i,[p, k]}\right)^{2} \geq \frac{c_{p}}{n}\|\mathbf{y}\|^{2},
$$

where $c_{p}$ is a constant depending only on $p$. 
- All the B-splines, except for the first $k+1$ and the last $k+1$, are uniformly shifted-scaled versions of $p-k$ fixed reference functions $\beta_{1,[p, k]}, \ldots, \beta_{p-k,[p, k]}$, namely the first $p-k$ $B$-splines defined on the reference knot sequence

$$
\underbrace{0, \ldots, 0}_{p-k}, \underbrace{1, \ldots, 1}_{p-k}, \ldots, \underbrace{\eta, \ldots, \eta}_{p-k}, \quad \eta=\left\lceil\frac{p+1}{p-k}\right\rceil .
$$

The precise formula we shall need later on is the following: setting

$$
\nu=\left\lceil\frac{k+1}{p-k}\right\rceil
$$

for the B-splines $B_{k+2,[p, k]}, \ldots, B_{k+1+(n-\nu)(p-k),[p, k]}$ we have

$$
\begin{aligned}
& B_{k+1+(p-k)(r-1)+q,[p, k]}(x)=\beta_{q,[p, k]}(n x-r+1), \\
& r=1, \ldots, n-\nu, \quad q=1, \ldots, p-k .
\end{aligned}
$$

We point out that the supports of the reference B-splines $\beta_{q,[p, k]}$ satisfy

$$
\operatorname{supp}\left(\beta_{1,[p, k]}\right) \subseteq \operatorname{supp}\left(\beta_{2,[p, k]}\right) \subseteq \ldots \subseteq \operatorname{supp}\left(\beta_{p-k,[p, k]}\right)=[0, \eta] .
$$

Figures 6.2 and 6.3 display the graphs of the B-splines $B_{1,[p, k]}, \ldots, B_{n(p-k)+k+1,[p, k]}$ for the degree $p=3$ and the smoothness $k=1$, and the graphs of the associated reference B-splines $\beta_{1,[p, k]}, \beta_{2,[p, k]}$.

The basis functions $\varphi_{1}, \ldots, \varphi_{N}$ are defined as follows:

$$
\varphi_{i}=B_{i+1,[p, k]}, \quad i=1, \ldots, n(p-k)+k-1 .
$$

In particular, we have $N=n(p-k)+k-1$ and $\mathcal{W}=\operatorname{span}\left(\varphi_{1}, \ldots, \varphi_{N}\right)=\mathcal{W}_{n,[p, k]}$.

GLT analysis of the higher-order FE discretization matrices. The stiffness matrix (6.13) resulting from the choice of the basis functions as in (6.23) will be denoted by $A_{n,[p, k]}(a)$,

$$
A_{n,[p, k]}(a)=\left[\int_{0}^{1} a(x) B_{j+1,[p, k]}^{\prime}(x) B_{i+1,[p, k]}^{\prime}(x) \mathrm{d} x\right]_{i, j=1}^{n(p-k)+k-1} .
$$

The main result of this section is Theorem 6.5, which gives the spectral distribution of the normalized sequence $\left\{n^{-1} A_{n,[p, k]}(a)\right\}_{n}$. The proof of Theorem 6.5 requires Lemma 6.4, which provides an approximate construction of the matrix $A_{n,[p, k]}(1)$ corresponding to the constant-coefficient case where $a(x)=1$ identically. In view of what follows, define the $(p-k) \times(p-k)$ blocks

$$
K_{[p, k]}^{[\ell]}=\left[\int_{\mathbb{R}} \beta_{j,[p, k]}^{\prime}(t) \beta_{i,[p, k]}^{\prime}(t-\ell) \mathrm{d} t\right]_{i, j=1}^{p-k}, \quad \ell \in \mathbb{Z},
$$

and the $(p-k) \times(p-k)$ matrix-valued function $\kappa_{[p, k]}:[-\pi, \pi] \rightarrow \mathbb{C}^{(p-k) \times(p-k)}$,

$$
\kappa_{[p, k]}(\theta)=\sum_{\ell \in \mathbb{Z}} K_{[p, k]}^{[\ell]} e^{\mathrm{i} \ell \theta}=K_{[p, k]}^{[0]}+\sum_{\ell>0}\left(K_{[p, k]}^{[\ell]} e^{\mathrm{i} \ell \theta}+\left(K_{[p, k]}^{[\ell]}\right)^{T} e^{-\mathrm{i} \ell \theta}\right) .
$$

Due to the compact support of the reference B-splines $\beta_{1,[p, k]}, \ldots, \beta_{p-k,[p, k]}$ (see (6.22)), there is only a finite number of nonzero blocks $K_{[p, k]}^{[\ell]}$ and, consequently, the series in (6.25) is actually a finite sum.

NOTATION 6.3. From now on, we will use the following notation. 


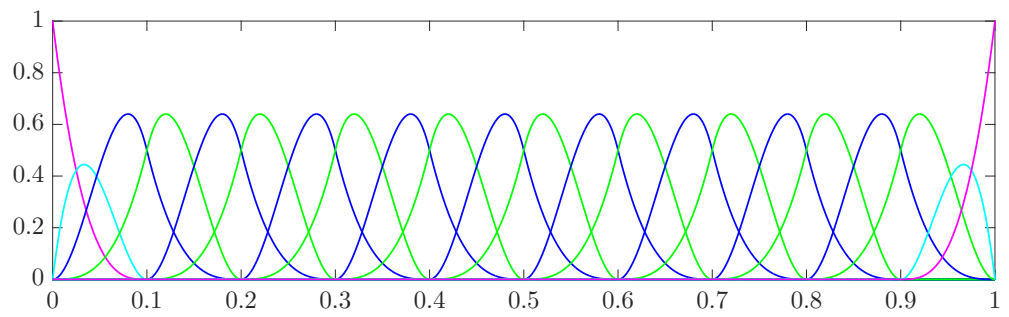

FIG. 6.2. B-splines $B_{1,[p, k]}, \ldots, B_{n(p-k)+k+1,[p, k]}$ for $p=3$ and $k=1$, with $n=10$.

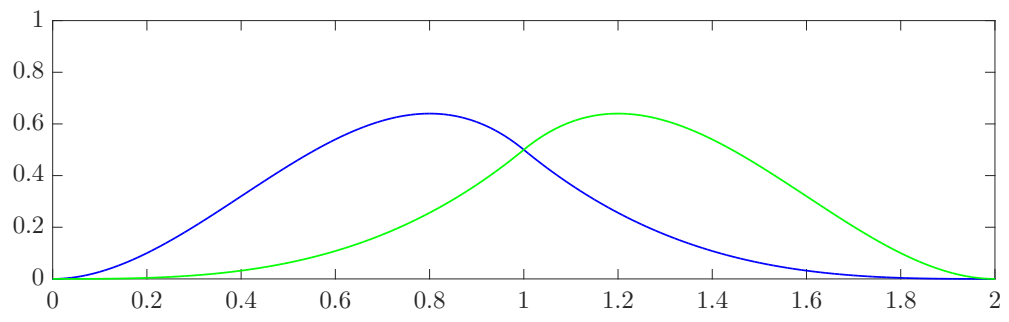

FIG. 6.3. Reference B-splines $\beta_{1,[p, k]}, \beta_{2,[p, k]}$ for $p=3$ and $k=1$.

- If $p, n \geq 1,0 \leq k \leq p-1$, and $X$ is a matrix of size $n(p-k)+k-1$, we denote by $\tilde{X}$ the principal submatrix of $X$ corresponding to the row and column indices $i, j=k+1, \ldots, k+(n-\nu)(p-k)$, where $\nu=\lceil(k+1) /(p-k)\rceil$ as in (6.20).

- If $p, n \geq 1,0 \leq k \leq p-1$, and $X$ is a matrix of size $n(p-k)+k-1$, we denote by $\hat{X}$ the block diagonal matrix

$$
\hat{X}=\left[\begin{array}{ccc}
I_{k(p-k)-k} & & \\
& X & \\
& & 1
\end{array}\right]=I_{k(p-k)-k} \oplus X \oplus[1],
$$

where it is understood that the block $I_{k(p-k)-k}$ is not present if $k(p-k)-k=0$, i.e., if $k=0$ or $k=p-1$. Note that $\hat{X}$ has the following key properties:

- its size $(n+k)(p-k)$ is a multiple of $p-k$, and it is such that the difference $(n+k)(p-k)-(n(p-k)+k-1)=k(p-k)-k+1>0$ is independent of $n$;

- it contains $X$ as a principal submatrix in such a way that $\tilde{X}$ is the principal submatrix of $\hat{X}$ corresponding to the row and column indices $i, j=k(p-k)+1, \ldots$, $k(p-k)+(n-\nu)(p-k)$;

- it satisfies the matrix identity $X=P_{n,[p, k]}^{*} \hat{X} P_{n,[p, k]}$, where $P_{n,[p, k]}$ is the $(n+k)(p-k) \times(n(p-k)+k-1)$ matrix given by

$$
P_{n,[p, k]}=\left[\frac{O}{I_{n(p-k)+k-1}} \mathbf{0}^{T}\right] ;
$$

- its eigenvalues (respectively, singular values) are given by the eigenvalues (respectively, singular values) of $X$ plus further $k(p-k)-k+1$ eigenvalues (respectively, singular values) that are equal to 1 .

LEMMA 6.4. Let $p, n \geq 1$ and $0 \leq k \leq p-1$. Then

$$
\tilde{A}_{n,[p, k]}(1)=n T_{n-\nu}\left(\kappa_{[p, k]}\right) .
$$


Proof. By (6.15) and (6.21), for all $r, R=1, \ldots, n-\nu$, and $q, Q=1, \ldots, p-k$, we have

$$
\begin{aligned}
& \left(\tilde{A}_{n,[p, k]}(1)\right)_{(p-k)(r-1)+q,(p-k)(R-1)+Q} \\
& \quad=\int_{0}^{1} B_{k+1+(p-k)(R-1)+Q,[p, k]}^{\prime}(x) B_{k+1+(p-k)(r-1)+q,[p, k]}^{\prime}(x) \mathrm{d} x \\
& =\int_{\mathbb{R}} B_{k+1+(p-k)(R-1)+Q,[p, k]}^{\prime}(x) B_{k+1+(p-k)(r-1)+q,[p, k]}^{\prime}(x) \mathrm{d} x \\
& =n^{2} \int_{\mathbb{R}} \beta_{Q,[p, k]}^{\prime}(n x-R+1) \beta_{q,[p, k]}^{\prime}(n x-r+1) \mathrm{d} x \\
& \quad=n \int_{\mathbb{R}} \beta_{Q,[p, k]}^{\prime}(y) \beta_{q,[p, k]}^{\prime}(y-r+R) \mathrm{d} y
\end{aligned}
$$

and

$$
\begin{aligned}
\left(T_{n-\nu}\left(\kappa_{[p, k]}\right)\right)_{(p-k)(r-1)+q,(p-k)(R-1)+Q} & =\left(K_{[p, k]}^{[r-R]}\right)_{q, Q} \\
& =\int_{\mathbb{R}} \beta_{Q,[p, k]}^{\prime}(y) \beta_{q,[p, k]}^{\prime}(y-r+R) \mathrm{d} y,
\end{aligned}
$$

which completes the proof.

THEOREM 6.5. Let $a \in L^{1}([0,1]), p \geq 1$, and $0 \leq k \leq p-1$. Then

$$
\left\{n^{-1} A_{n,[p, k]}(a)\right\}_{n} \sim_{\sigma, \lambda} a(x) \kappa_{[p, k]}(\theta) .
$$

Proof. We first note that it is enough to prove (6.26) with $A_{n,[p, k]}(a)$ replaced by $\hat{A}_{n,[p, k]}(a)$ because we have observed in Notation 6.3 that, except for $k(p-k)-k+1$ additional singular values (respectively, eigenvalues) that are equal to 1 , the singular values (respectively, eigenvalues) of $\hat{A}_{n,[p, k]}(a)$ are the same as the singular values (respectively, eigenvalues) of $A_{n,[p, k]}(a)$. In view of GLT 1 and the symmetry of $\hat{A}_{n,[p, k]}(a)$, in order to prove (6.26) with $A_{n,[p, k]}(a)$ replaced by $\hat{A}_{n,[p, k]}(a)$, it is enough to show that

$$
\left\{n^{-1} \hat{A}_{n,[p, k]}(a)\right\}_{n} \sim_{\mathrm{GLT}} a(x) \kappa_{[p, k]}(\theta) .
$$

The proof of (6.27) consists of the following three steps.

Step 1. We first prove (6.27) in the constant-coefficient case where $a(x)=1$ identically. In this case, by Lemma $6.4, n^{-1} \tilde{A}_{n,[p, k]}(1)=T_{n-\nu}\left(\kappa_{[p, k]}\right)$. Considering that $n^{-1} \tilde{A}_{n,[p, k]}(1)$ is the principal submatrix of $n^{-1} \hat{A}_{n,[p, k]}(1)$ corresponding to the row and column indices $i, j=k(p-k)+1, \ldots, k(p-k)+(n-\nu)(p-k)$, we infer that

$$
n^{-1} \hat{A}_{n,[p, k]}(1)=T_{n+k}\left(\kappa_{[p, k]}\right)+R_{n,[p, k]}, \quad \operatorname{rank}\left(R_{n,[p, k]}\right) \leq 2(p-k)(k+\nu) .
$$

Hence, the desired relation $\left\{n^{-1} \hat{A}_{n,[p, k]}(1)\right\}_{n} \sim_{\mathrm{GLT}} \kappa_{[p, k]}(\theta)$ follows from Z 1, GLT 3, and GLT 4.

Step 2. Now we prove (6.27) in the case where $a \in C([0,1])$. Let

$$
Z_{n,[p, k]}(a)=n^{-1} \hat{A}_{n,[p, k]}(a)-n^{-1} D_{n+k}\left(a I_{p-k}\right) \hat{A}_{n,[p, k]}(1) .
$$

By (6.14), (6.15), and (6.18), for all $r, R=1, \ldots, n-\nu$, and $q, Q=1, \ldots, p-k$, we have

$$
\left|\left(n \tilde{Z}_{n,[p, k]}(a)\right)_{(p-k)(r-1)+q,(p-k)(R-1)+Q}\right|
$$




$$
\begin{aligned}
& =\mid\left(\tilde{A}_{n,[p, k]}(a)\right)_{(p-k)(r-1)+q,(p-k)(R-1)+Q} \\
& -\left(\left(\operatorname{diag}_{i=k+1, \ldots, k+n-\nu} a\left(\frac{i}{n+k}\right) I_{p-k}\right) \tilde{A}_{n,[p, k]}(1)\right)_{(p-k)(r-1)+q,(p-k)(R-1)+Q} \\
& =\mid \int_{0}^{1}\left[a(x)-a\left(\frac{k+r}{n+k}\right)\right] \\
& \text { - } B_{k+1+(p-k)(R-1)+Q,[p, k]}^{\prime}(x) B_{k+1+(p-k)(r-1)+q,[p, k]}^{\prime}(x) \mathrm{d} x \mid \\
& =\mid \int_{\tau_{k+1+(p-k)(r-1)+q}}^{\tau_{k+1+(p-k)(r-1)+q+p+1}}\left[a(x)-a\left(\frac{k+r}{n+k}\right)\right] \\
& \text { - } B_{k+1+(p-k)(R-1)+Q,[p, k]}^{\prime}(x) B_{k+1+(p-k)(r-1)+q,[p, k]}^{\prime}(x) \mathrm{d} x \\
& \leq C_{p}^{2} n^{2} \int_{(r-1) / n}^{(r+p) / n}\left|a(x)-a\left(\frac{k+r}{n+k}\right)\right| \mathrm{d} x \leq C_{p}^{2}(p+1) n \omega_{a}\left(\frac{2 p}{n}\right)
\end{aligned}
$$

where $\omega_{a}(\cdot)$ is the modulus of continuity of $a$ and the last inequality is justified by the fact that the maximum distance of a point $x$ in the interval $[(r-1) / n,(r+p) / n]$ from the point $(k+r) /(n+k)$ is not larger than $2 p / n$. It follows that each entry of $\tilde{Z}_{n,[p, k]}(a)$ is bounded in modulus by $D_{p} \omega_{a}(1 / n)$, where $D_{p}$ is a constant depending only on $p$. Moreover, by (6.15), the matrix $\tilde{Z}_{n,[p, k]}(a)$ is banded with bandwidth bounded by a constant $w_{p}$ depending only on $p$. Thus, by $\mathbf{N} \mathbf{1},\left\|\tilde{Z}_{n,[p, k]}(a)\right\| \leq w_{p} D_{p} \omega_{a}(1 / n) \rightarrow 0$ as $n \rightarrow \infty$. Considering that $\tilde{Z}_{n,[p, k]}(a)$ is the principal submatrix of $Z_{n,[p, k]}(a)$ corresponding to the row and column indices $i, j=k(p-k)+1, \ldots, k(p-k)+(n-\nu)(p-k)$, we arrive at

$$
Z_{n,[p, k]}(a)=N_{n,[p, k]}+R_{n,[p, k]}
$$

where $\left\|N_{n,[p, k]}\right\|=\left\|\tilde{Z}_{n,[p, k]}(a)\right\| \rightarrow 0$ as $n \rightarrow \infty$ and $\operatorname{rank}\left(R_{n,[p, k]}\right) \leq 2(p-k)(k+\nu)$. It follows from $\mathbf{Z} \mathbf{1}$ that $\left\{Z_{n,[p, k]}(a)\right\}_{n}$ is zero-distributed. Since

$$
n^{-1} \hat{A}_{n,[p, k]}(a)=n^{-1} D_{n+k}\left(a I_{p-k}\right) \hat{A}_{n,[p, k]}(1)+Z_{n,[p, k]}(a)
$$

we conclude that $\left\{n^{-1} \hat{A}_{n,[p, k]}(a)\right\}_{n} \sim_{\mathrm{GLT}} a(x) \kappa_{[p, k]}(\theta)$ by Step 1, GLT 3, and GLT 4.

Step 3. Finally, we prove (6.27) in the general case where $a \in L^{1}([0,1])$. By the density of $C([0,1])$ in $L^{1}([0,1])$, there exist functions $a_{m} \in C([0,1])$ such that $a_{m} \rightarrow a$ in $L^{1}([0,1])$. By Step 2,

$$
\left\{n^{-1} \hat{A}_{n,[p, k]}\left(a_{m}\right)\right\}_{n} \sim_{\mathrm{GLT}} a_{m}(x) \kappa_{[p, k]}(\theta) .
$$

Moreover,

$$
a_{m}(x) \kappa_{[p, k]}(\theta) \rightarrow a(x) \kappa_{[p, k]}(\theta) \text { in measure. }
$$

We show that

$$
\left\{n^{-1} \hat{A}_{n,[p, k]}\left(a_{m}\right)\right\}_{n} \stackrel{\text { a.c.s. }}{\longrightarrow}\left\{n^{-1} \hat{A}_{n,[p, k]}(a)\right\}_{n} .
$$


ETNA

Kent State University and

Johann Radon Institute (RICAM)

Once this is done, the thesis (6.27) follows immediately from GLT 7. To prove (6.28), we note that, by $\mathbf{N} \mathbf{3}$ and (6.18),

$$
\begin{aligned}
& \left\|\hat{A}_{n,[p, k]}(a)-\hat{A}_{n,[p, k]}\left(a_{m}\right)\right\|_{1}=\left\|A_{n,[p, k]}(a)-A_{n,[p, k]}\left(a_{m}\right)\right\|_{1} \\
& \quad \leq \sum_{i, j=1}^{n(p-k)+k-1}\left|\int_{0}^{1}\left[a(x)-a_{m}(x)\right] B_{j+1,[p, k]}^{\prime}(x) B_{i+1,[p, k]}^{\prime}(x) \mathrm{d} x\right| \\
& \quad \leq \int_{0}^{1}\left|a(x)-a_{m}(x)\right| \sum_{i, j=1}^{n(p-k)+k-1}\left|B_{j+1,[p, k]}^{\prime}(x)\right|\left|B_{i+1,[p, k]}^{\prime}(x)\right| \mathrm{d} x \\
& \quad \leq C_{p}^{2} n^{2}\left\|a-a_{m}\right\|_{L^{1}} .
\end{aligned}
$$

Thus, the a.c.s. convergence (6.28) follows from ACS 6.

REMARK 6.6 (Space-time higher-order FE-DG discretization of time-dependent diffusion equations). Consider the time-dependent diffusion equation

$$
\begin{cases}\partial_{t} u(t, x)-\partial_{x}\left(a(x) \partial_{x} u(t, x)\right)=f(t, x), & (t, x) \in(0, T) \times(0,1), \\ u(t, 0)=u(t, 1)=0, & t \in(0, T), \\ u(0, x)=0, & x \in(0,1) .\end{cases}
$$

If we discretize (6.29) by the space-time higher-order FE-DG approximation technique considered in [11], then the resulting (normalized) FE-DG discretization matrices enjoy an asymptotic spectral distribution described by a $(q+1)(p-k) \times(q+1)(p-k)$ matrix-valued function. This result was proved in [11, Theorem A.6] by a direct (complicated and cumbersome) approach. By following step by step the proof of Theorem 6.5, we can give an alternative (much more lucid and simpler) proof of [11, Theorem A.6] based on the theory of block GLT sequences.

REMARK 6.7 (Formal structure of the symbol). From a formal point of view (i.e., disregarding the regularity of $a(x)$ and $u(x)$ ), problem (6.12) can be rewritten in the form

$$
\left\{\begin{array}{l}
-a(x) u^{\prime \prime}(x)-a^{\prime}(x) u^{\prime}(x)=f(x), \quad x \in(0,1), \\
u(0)=u(1)=0 .
\end{array}\right.
$$

From this reformulation, it appears more clearly that the (singular value and spectral) symbol $a(x) \kappa_{[p, k]}(\theta)$ consists of the following two "ingredients".

- The coefficient of the higher-order differential operator, namely $a(x)$, in the physical variable $x$. To make a parallelism with Hörmander's theory [60], the higher-order differential operator $-a(x) u^{\prime \prime}(x)$ is the so-called principal symbol of the complete differential operator $-a(x) u^{\prime \prime}(x)-a^{\prime}(x) u^{\prime}(x)$ and $a(x)$ is then the coefficient of the principal symbol.

- The trigonometric polynomial associated with the FE discretization of the higher-order derivative $-u^{\prime \prime}(x)$, namely $\kappa_{[p, k]}(\theta)$, in the Fourier variable $\theta$. To see that $\kappa_{[p, k]}(\theta)$ is really the trigonometric polynomial associated with the FE discretization of $-u^{\prime \prime}(x)$, simply note that $\kappa_{[p, k]}(\theta)$ is the (singular value and spectral) symbol of the sequence of FE matrices $\left\{n^{-1} A_{n,[p, k]}(1)\right\}_{n}$, which arises from the FE discretization of the Poisson problem

$$
\left\{\begin{array}{l}
-u^{\prime \prime}(x)=f(x), \quad x \in(0,1), \\
u(0)=u(1)=0 .
\end{array}\right.
$$

We observe that the term $-a^{\prime}(x) u^{\prime}(x)$, which only depends on lower-order derivatives of $u(x)$, does not enter the expression of the symbol.

EXAMPLE 6.8. Let $\lambda_{1,2}(x, \theta)$ be the two eigenvalue functions of $a(x) \kappa_{[p, k]}(\theta)$. Figure 6.4 


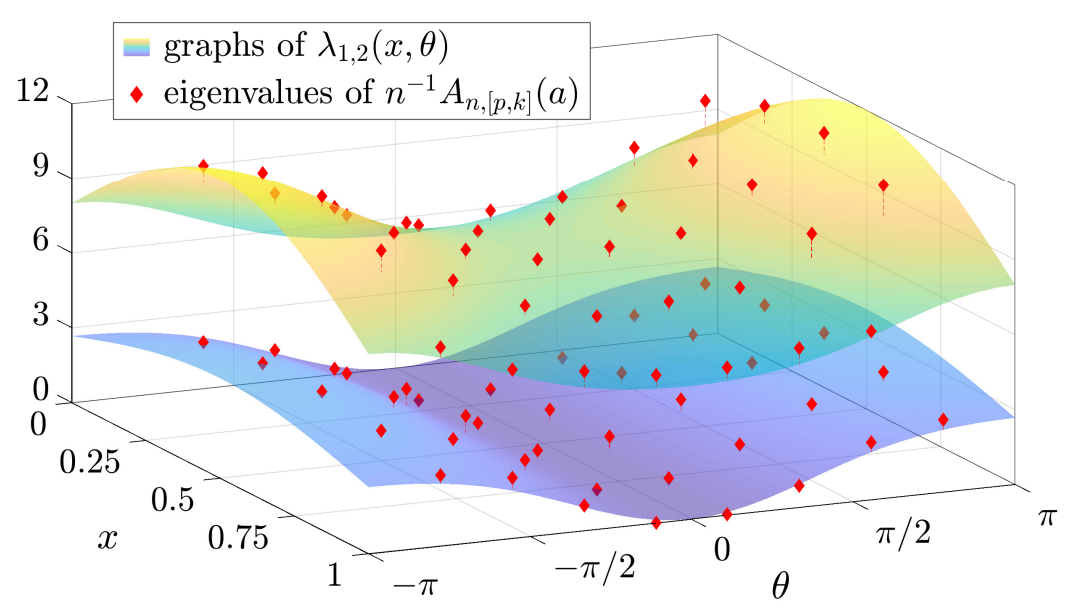

FIG. 6.4. Comparison between the spectrum of $n^{-1} A_{n,[p, k]}(a)$ and the two eigenvalue functions of the symbol $a(x) \kappa_{[p, k]}(\theta)$ for $a(x)=2+\sin (\pi x)$ and $p=2, k=0, n=40$.

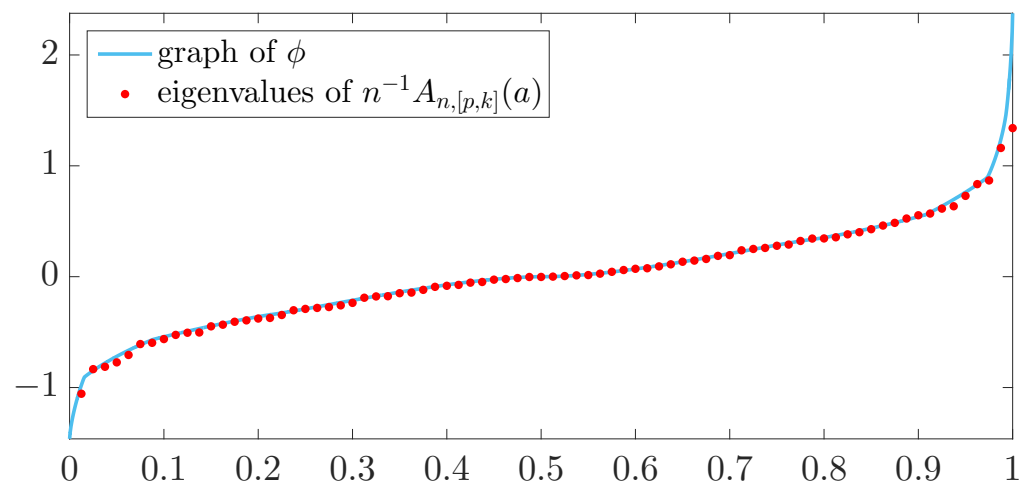

FIG. 6.5. Comparison between the spectrum of $n^{-1} A_{n,[p, k]}(a)$ and the rearranged version $\phi$ of the symbol $a(x) \kappa_{[p, k]}(\theta)$ for $a(x)=e^{-x}(1-2 x-\sin (7 x))$ and $p=3, k=1, n=40$.

displays the graphs of $\lambda_{1,2}(x, \theta)$ and the eigenvalues $\lambda_{1}, \ldots, \lambda_{n(p-k)+k-1}$ of $n^{-1} A_{n,[p, k]}(a)$ for $a(x)=2+\sin (\pi x)$ and $p=2, k=0, n=40$. The eigenvalues of $n^{-1} A_{n,[p, k]}(a)$ have been plotted over a uniform grid on the domain $[0,1] \times[-\pi, \pi]$. We clearly see from the figure an excellent agreement between $\lambda_{1,2}(x, \theta)$ and the eigenvalues of $n^{-1} A_{n,[p, k]}(a)$, as predicted in Chapter 5 (fourth paragraph).

EXAMPLE 6.9. Let $\phi$ be the canonical rearranged version of $a(x) \kappa_{[p, k]}(\theta)$ obtained as the limit of the piecewise linear functions $\phi_{\rho}$, according to the construction in Chapter 5 (fifth paragraph). Figure 6.5 displays the graph of $\phi$ and the eigenvalues $\lambda_{1}, \ldots, \lambda_{n(p-k)+k-1}$ of $n^{-1} A_{n,[p, k]}(a)$ for $a(x)=e^{-x}(1-2 x-\sin (7 x))$ and $p=3, k=1, n=40$. The graph of $\phi$ has been obtained by plotting the graph of $\phi_{\rho}$ corresponding to a large value of $\rho$. The eigenvalues of $n^{-1} A_{n,[p, k]}(a)$ have been sorted in non-decreasing order and placed at the points $\left(t_{q}, \lambda_{q}\right)$ with $t_{q}=\frac{q}{n(p-k)+k-1}, q=1, \ldots, n(p-k)+k-1$. We clearly see from the figure an excellent agreement between $\phi$ and the eigenvalues of $n^{-1} A_{n,[p, k]}(a)$. 
6.3. Higher-order FE discretization of convection-diffusion-reaction equations. Suppose we add to the diffusion equation (6.12) a convection and a reaction term. In this way, we obtain the following convection-diffusion-reaction problem:

$$
\left\{\begin{array}{l}
-\left(a(x) u^{\prime}(x)\right)^{\prime}+b(x) u^{\prime}(x)+c(x) u(x)=f(x), \quad x \in(0,1), \\
u(0)=u(1)=0 .
\end{array}\right.
$$

Based on Remark 6.7, we expect that the term $b(x) u^{\prime}(x)+c(x) u(x)$, which only involves lower-order derivatives of $u(x)$, does not enter the expression of the symbol. In other words, if we consider for problem (6.30) the same higher-order FE discretization as in Section 6.2, then the symbol of the resulting sequence of (normalized) FE discretization matrices should be again $a(x) \kappa_{[p, k]}(\theta)$ as per Theorem 6.5. We are going to show that this is in fact the case.

FE discretization. The weak form of (6.30) reads as follows [25, Chapter 8]: find $u \in H_{0}^{1}([0,1])$ such that

$$
\mathrm{a}(u, w)=\mathrm{f}(w), \quad \forall w \in H_{0}^{1}([0,1]),
$$

where

$$
\begin{aligned}
\mathrm{a}(u, w) & =\int_{0}^{1} a(x) u^{\prime}(x) w^{\prime}(x) \mathrm{d} x+\int_{0}^{1} b(x) u^{\prime}(x) w(x) \mathrm{d} x+\int_{0}^{1} c(x) u(x) w(x) \mathrm{d} x, \\
\mathrm{f}(w) & =\int_{0}^{1} f(x) w(x) \mathrm{d} x .
\end{aligned}
$$

In the FE method, we fix a set of basis functions $\left\{\varphi_{1}, \ldots, \varphi_{N}\right\} \subset H_{0}^{1}([0,1])$, and we look for an approximation of the exact solution in the space $\mathcal{W}=\operatorname{span}\left(\varphi_{1}, \ldots, \varphi_{N}\right)$ by solving the following discrete problem: find $u_{\mathcal{W}} \in \mathcal{W}$ such that

$$
\mathrm{a}\left(u_{\mathcal{W}}, w\right)=\mathrm{f}(w), \quad \forall w \in \mathcal{W} .
$$

Since $\left\{\varphi_{1}, \ldots, \varphi_{N}\right\}$ is a basis for $\mathcal{W}$, we can write $u_{\mathcal{W}}=\sum_{j=1}^{N} u_{j} \varphi_{j}$ for a unique vector $\mathbf{u}=\left(u_{1}, \ldots, u_{N}\right)^{T}$. By linearity, the computation of $u_{\mathcal{W}}$ (i.e., of $\mathbf{u}$ ) reduces to solving the linear system

$$
S \mathbf{u}=\mathbf{f},
$$

where $\mathbf{f}=\left(\mathrm{f}\left(\varphi_{1}\right), \ldots, \mathrm{f}\left(\varphi_{N}\right)\right)^{T}$ and $S$ is the stiffness matrix,

$$
S=\left[\mathrm{a}\left(\varphi_{j}, \varphi_{i}\right)\right]_{i, j=1}^{N} .
$$

Note that $S$ admits the following decomposition:

$$
S=A+Z
$$

where

$$
A=\left[\int_{0}^{1} a(x) \varphi_{j}^{\prime}(x) \varphi_{i}^{\prime}(x) \mathrm{d} x\right]_{i, j=1}^{N}
$$

is the (symmetric) diffusion matrix and

$$
Z=\left[\int_{0}^{1} b(x) \varphi_{j}^{\prime}(x) \varphi_{i}(x) \mathrm{d} x\right]_{i, j=1}^{N}+\left[\int_{0}^{1} c(x) \varphi_{j}(x) \varphi_{i}(x) \mathrm{d} x\right]_{i, j=1}^{N}
$$


is the sum of the convection and reaction matrices.

GLT analysis of the higher-order FE discretization matrices. Following the higher-order FE approach as in Section 6.2, the basis functions $\varphi_{1}, \ldots, \varphi_{N}$ are chosen as in (6.23). The stiffness matrix resulting from this choice will be denoted by $S_{n,[p, k]}(a, b, c)$. According to (6.31), it can be decomposed as follows:

$$
S_{n,[p, k]}(a, b, c)=A_{n,[p, k]}(a)+Z_{n,[p, k]}(b, c),
$$

where

$$
\begin{aligned}
A_{n,[p, k]}(a)= & {\left[\int_{0}^{1} a(x) B_{j+1,[p, k]}^{\prime}(x) B_{i+1,[p, k]}^{\prime}(x) \mathrm{d} x\right]_{i, j=1}^{n(p-k)+k-1} } \\
Z_{n,[p, k]}(b, c)= & {\left[\int_{0}^{1} b(x) B_{j+1,[p, k]}^{\prime}(x) B_{i+1,[p, k]}(x) \mathrm{d} x\right]_{i, j=1}^{n(p-k)+k-1} } \\
& +\left[\int_{0}^{1} c(x) B_{j+1,[p, k]}(x) B_{i+1,[p, k]}(x) \mathrm{d} x\right]_{i, j=1}^{n(p-k)+k-1}
\end{aligned}
$$

Note that $A_{n,[p, k]}(a)$ is the same as in (6.24) and Theorem 6.5. The main result of this section is Theorem 6.10, which shows that Theorem 6.5 holds unchanged with $S_{n,[p, k]}(a, b, c)$ in place of $A_{n,[p, k]}(a)$. This highlights a general aspect: lower-order terms such as $b(x) u^{\prime}(x)+c(x) u(x)$ do not enter the expression of the symbol and do not affect in any way the asymptotic singular value and eigenvalue distribution of DE discretization matrices.

THEOREM 6.10. Let $a, b, c \in L^{1}([0,1]), p \geq 1$, and $0 \leq k \leq p-1$. Then

$$
\left\{n^{-1} S_{n,[p, k]}(a, b, c)\right\}_{n} \sim_{\sigma, \lambda} a(x) \kappa_{[p, k]}(\theta),
$$

where $\kappa_{[p, k]}(\theta)$ is defined in (6.25).

Proof. Throughout this proof, we make use of Notation 6.3, and we use the letter $C$ to denote a generic constant independent of $n$. We are going to show that

$$
\left\|Z_{n,[p, k]}(b, c)\right\|_{2} \leq C n \text {. }
$$

Once this is done, the thesis is proved. Indeed, by (6.33) and the definition of $\hat{Z}_{n,[p, k]}(b, c)$ (see Notation 6.3), we immediately obtain

$$
\left\|\hat{Z}_{n,[p, k]}(b, c)\right\|_{2} \leq C n
$$

which in turn implies that $\left\{n^{-1} \hat{Z}_{n,[p, k]}(b, c)\right\}_{n}$ is zero-distributed by $\mathbf{Z 2}$. In view of the decomposition

$$
n^{-1} \hat{S}_{n,[p, k]}(a, b, c)=n^{-1} \hat{A}_{n,[p, k]}(a)+n^{-1} \hat{Z}_{n,[p, k]}(b, c)
$$

(see (6.32)), by (6.27), GLT 3, and GLT 4, we get $\left\{n^{-1} \hat{S}_{n,[p, k]}(a, b, c)\right\}_{n} \sim_{\mathrm{GLT}} a(x) \kappa_{[p, k]}(\theta)$. Thus, $\left\{n^{-1} \hat{S}_{n,[p, k]}(a, b, c)\right\}_{n} \sim_{\sigma, \lambda} a(x) \kappa_{[p, k]}(\theta)$ by GLT 1 and GLT 2, which immediately implies that $\left\{n^{-1} S_{n,[p, k]}(a, b, c)\right\}_{n} \sim_{\sigma, \lambda} a(x) \kappa_{[p, k]}(\theta)$.

It only remains to prove (6.33). Using $\mathbf{N} \mathbf{3}$ and (6.16)-(6.18), we obtain

$$
\left\|Z_{n,[p, k]}(b, c)\right\|_{2} \leq\left\|Z_{n,[p, k]}(b, c)\right\|_{1} \leq \sum_{i, j=1}^{n(p-k)+k-1}\left|\left(Z_{n,[p, k]}(b, c)\right)_{i j}\right|
$$




$$
\begin{aligned}
= & \sum_{i, j=1}^{n(p-k)+k-1} \mid \int_{0}^{1} b(x) B_{j+1,[p, k]}^{\prime}(x) B_{i+1,[p, k]}(x) \mathrm{d} x \\
& \quad+\int_{0}^{1} c(x) B_{j+1,[p, k]}(x) B_{i+1,[p, k]}(x) \mathrm{d} x \mid \\
\leq & \int_{0}^{1}|b(x)| \sum_{j=1}^{n(p-k)+k-1}\left|B_{j+1,[p, k]}^{\prime}(x)\right| \sum_{i=1}^{n(p-k)+k-1}\left|B_{i+1,[p, k]}(x)\right| \mathrm{d} x \\
& +\int_{0}^{1}|c(x)| \sum_{j=1}^{n(p-k)+k-1}\left|B_{j+1,[p, k]}(x)\right| \sum_{i=1}^{n(p-k)+k-1}\left|B_{i+1,[p, k]}(x)\right| \mathrm{d} x \\
\leq & C_{p} n\|b\|_{L^{1}}+\|c\|_{L^{1}} \leq C n,
\end{aligned}
$$

and (6.33) is proved.

6.4. Higher-order FE discretization of systems of DEs. Consider again the same system of DEs as in Section 6.1, i.e.,

$$
\left\{\begin{aligned}
-a_{11}(x) u_{1}^{\prime \prime}(x)+a_{12}(x) u_{2}^{\prime}(x)=f_{1}(x), & x \in(0,1), \\
a_{21}(x) u_{1}^{\prime}(x)+a_{22}(x) u_{2}(x)=f_{2}(x), & x \in(0,1), \\
u_{1}(0)=0, \quad u_{1}(1)=0 & \\
u_{2}(0)=0, \quad u_{2}(1)=0 . &
\end{aligned}\right.
$$

In this section we consider the higher-order FE discretization of (6.34). Through the theory of block GLT sequences we show that, under suitable assumptions on the DE coefficients $a_{11}, a_{12}, a_{21}, a_{22}$, the corresponding sequence of (normalized) $\mathrm{FE}$ discretization matrices enjoys a spectral distribution described by a $2(p-k) \times 2(p-k)$ matrix-valued function, where $p$ and $k$ are, respectively, the degree and the smoothness of the piecewise polynomial functions involved in the FE approximation, while the number 2 in front of $(p-k)$ coincides with the number of equations that compose the system (6.34).

FE discretization. The weak form of (6.34) reads as follows: find $u_{1}, u_{2} \in H_{0}^{1}([0,1])$ such that, for all $w \in H_{0}^{1}([0,1])$,

$$
\left\{\begin{array}{l}
\int_{0}^{1} a_{11}(x) u_{1}^{\prime}(x) w^{\prime}(x) \mathrm{d} x+\int_{0}^{1} a_{12}(x) u_{2}^{\prime}(x) w(x) \mathrm{d} x=\int_{0}^{1} f_{1}(x) w(x) \mathrm{d} x, \\
\int_{0}^{1} a_{21}(x) u_{1}^{\prime}(x) w(x) \mathrm{d} x+\int_{0}^{1} a_{22}(x) u_{2}(x) w(x) \mathrm{d} x=\int_{0}^{1} f_{2}(x) w(x) \mathrm{d} x .
\end{array}\right.
$$

In the FE method, we fix a set of basis functions $\left\{\varphi_{1}, \ldots, \varphi_{N}\right\} \subset H_{0}^{1}([0,1])$, and we look for approximations $u_{1, \mathcal{W}}, u_{2, \mathcal{W}}$ of the exact solutions $u_{1}, u_{2}$ in the space $\mathcal{W}=\operatorname{span}\left(\varphi_{1}, \ldots, \varphi_{N}\right)$ by solving the following discrete problem: find $u_{1, \mathcal{W}}, u_{2, \mathcal{W}} \in \mathcal{W}$ such that, for all $w \in \mathcal{W}$,

$$
\left\{\begin{array}{c}
\int_{0}^{1} a_{11}(x) u_{1, \mathcal{W}}^{\prime}(x) w^{\prime}(x) \mathrm{d} x+\int_{0}^{1} a_{12}(x) u_{2, \mathcal{W}}^{\prime}(x) w(x) \mathrm{d} x=\int_{0}^{1} f_{1}(x) w(x) \mathrm{d} x, \\
\int_{0}^{1} a_{21}(x) u_{1, \mathcal{W}}^{\prime}(x) w(x) \mathrm{d} x+\int_{0}^{1} a_{22}(x) u_{2, \mathcal{W}}(x) w(x) \mathrm{d} x=\int_{0}^{1} f_{2}(x) w(x) \mathrm{d} x .
\end{array}\right.
$$

Since $\left\{\varphi_{1}, \ldots, \varphi_{N}\right\}$ is a basis of $\mathcal{W}$, we can write $u_{1, \mathcal{W}}=\sum_{j=1}^{N} u_{1, j} \varphi_{j}$ and $u_{2, \mathcal{W}}=\sum_{j=1}^{N} u_{2, j} \varphi_{j}$ for unique vectors $\mathbf{u}_{1}=\left(u_{1,1}, \ldots, u_{1, N}\right)^{T}$ and $\mathbf{u}_{2}=\left(u_{2,1}, \ldots, u_{2, N}\right)^{T}$. By linearity, the computation of $u_{1, \mathcal{W}}, u_{2, \mathcal{W}}$ (i.e., of $\mathbf{u}_{1}, \mathbf{u}_{2}$ ) reduces to solving the linear 
system

$$
S\left[\begin{array}{l}
\mathbf{u}_{1} \\
\mathbf{u}_{2}
\end{array}\right]=\left[\begin{array}{l}
\mathbf{f}_{1} \\
\mathbf{f}_{2}
\end{array}\right]
$$

where $\mathbf{f}_{1}=\left[\int_{0}^{1} f_{1}(x) \varphi_{i}(x) \mathrm{d} x\right]_{i=1}^{N}, \mathbf{f}_{2}=\left[\int_{0}^{1} f_{2}(x) \varphi_{i}(x) \mathrm{d} x\right]_{i=1}^{N}$, and $S$ is the stiffness matrix, which has the following block structure:

$$
S=\left[\begin{array}{ll}
A\left(a_{11}\right) & H\left(a_{12}\right) \\
H\left(a_{21}\right) & M\left(a_{22}\right)
\end{array}\right],
$$

where, for any $g \in L^{1}([0,1])$,

$$
\begin{aligned}
& A(g)=\left[\int_{0}^{1} g(x) \varphi_{j}^{\prime}(x) \varphi_{i}^{\prime}(x) \mathrm{d} x\right]_{i, j=1}^{N}, \\
& H(g)=\left[\int_{0}^{1} g(x) \varphi_{j}^{\prime}(x) \varphi_{i}(x) \mathrm{d} x\right]_{i, j=1}^{N}, \\
& M(g)=\left[\int_{0}^{1} g(x) \varphi_{j}(x) \varphi_{i}(x) \mathrm{d} x\right]_{i, j=1}^{N} .
\end{aligned}
$$

Note that, for any $\rho \neq 0$, the system (6.35) is equivalent to

$$
B^{(\rho)}\left[\begin{array}{l}
\mathbf{v}_{1} \\
\mathbf{v}_{2}
\end{array}\right]=\left[\begin{array}{c}
\rho^{-1} \mathbf{f}_{1} \\
\mathbf{f}_{2}
\end{array}\right],
$$

where $\mathbf{v}_{1}=\mathbf{u}_{1}, \mathbf{v}_{2}=\rho^{-1} \mathbf{u}_{2}$ and

$$
\begin{aligned}
B^{(\rho)} & =\left(\rho^{-1} I_{N} \oplus I_{N}\right) S\left(I_{N} \oplus \rho I_{N}\right) \\
& =\left[\begin{array}{cc}
\rho^{-1} I_{N} & O_{N} \\
O_{N} & I_{N}
\end{array}\right]\left[\begin{array}{ll}
A\left(a_{11}\right) & H\left(a_{12}\right) \\
H\left(a_{21}\right) & M\left(a_{22}\right)
\end{array}\right]\left[\begin{array}{ll}
I_{N} & O_{N} \\
O_{N} & \rho I_{N}
\end{array}\right] \\
& =\left[\begin{array}{cc}
\rho^{-1} A\left(a_{11}\right) & H\left(a_{12}\right) \\
H\left(a_{21}\right) & \rho M\left(a_{22}\right)
\end{array}\right] .
\end{aligned}
$$

GLT analysis of the higher-order FE discretization matrices. Following the higher-order FE approach as in Sections 6.2 and 6.3, the basis functions $\varphi_{1}, \ldots, \varphi_{N}$ are chosen as in (6.23). The stiffness matrix $S$ resulting from this choice and its normalized version $B^{(\rho)}$ corresponding to $\rho=n$ will be denoted by $S_{n,[p, k]}(A)$ and $B_{n,[p, k]}(A)$, respectively, where

$$
A=\left[\begin{array}{ll}
a_{11} & a_{12} \\
a_{21} & a_{22}
\end{array}\right] .
$$

According to (6.36)-(6.39) and (6.40), we have

$$
\begin{aligned}
S_{n,[p, k]}(A) & =\left[\begin{array}{ll}
A_{n,[p, k]}\left(a_{11}\right) & H_{n,[p, k]}\left(a_{12}\right) \\
H_{n,[p, k]}\left(a_{21}\right) & M_{n,[p, k]}\left(a_{22}\right)
\end{array}\right], \\
B_{n,[p, k]}(A) & =\left[\begin{array}{cc}
n^{-1} A_{n,[p, k]}\left(a_{11}\right) & H_{n,[p, k]}\left(a_{12}\right) \\
H_{n,[p, k]}\left(a_{21}\right) & n M_{n,[p, k]}\left(a_{22}\right)
\end{array}\right],
\end{aligned}
$$


where, for any $g \in L^{1}([0,1])$,

$$
\begin{aligned}
& A_{n,[p, k]}(g)=\left[\int_{0}^{1} g(x) B_{j+1,[p, k]}^{\prime}(x) B_{i+1,[p, k]}^{\prime}(x) \mathrm{d} x\right]_{i, j=1}^{n(p-k)+k-1}, \\
& H_{n,[p, k]}(g)=\left[\int_{0}^{1} g(x) B_{j+1,[p, k]}^{\prime}(x) B_{i+1,[p, k]}(x) \mathrm{d} x\right]_{i, j=1}^{n(p-k)+k-1}, \\
& M_{n,[p, k]}(g)=\left[\int_{0}^{1} g(x) B_{j+1,[p, k]}(x) B_{i+1,[p, k]}(x) \mathrm{d} x\right]_{i, j=1}^{n(p-k)+k-1}
\end{aligned}
$$

Note that $A_{n,[p, k]}(g)$ is the same as in (6.24) and Theorem 6.5 with the only difference that $a$ is replaced by $g$. The main result of this section is Theorem 6.14, which gives the spectral distribution of the sequence $\left\{B_{n,[p, k]}(A)\right\}_{n}$. In view of what follows, define the $(p-k) \times(p-k)$ blocks

$$
\begin{aligned}
& K_{[p, k]}^{[\ell]}=\left[\int_{\mathbb{R}} \beta_{j,[p, k]}^{\prime}(t) \beta_{i,[p, k]}^{\prime}(t-\ell) \mathrm{d} t\right]_{i, j=1}^{p-k}, \quad \ell \in \mathbb{Z}, \\
& H_{[p, k]}^{[\ell]}=\left[\int_{\mathbb{R}} \beta_{j,[p, k]}^{\prime}(t) \beta_{i,[p, k]}(t-\ell) \mathrm{d} t\right]_{i, j=1}^{p-k}, \quad \ell \in \mathbb{Z}, \\
& M_{[p, k]}^{[\ell]}=\left[\int_{\mathbb{R}} \beta_{j,[p, k]}(t) \beta_{i,[p, k]}(t-\ell) \mathrm{d} t\right]_{i, j=1}^{p-k}, \quad \ell \in \mathbb{Z},
\end{aligned}
$$

and the matrix-valued functions $\kappa_{[p, k]}, \xi_{[p, k]}, \mu_{[p, k]}:[-\pi, \pi] \rightarrow \mathbb{C}^{(p-k) \times(p-k)}$,

$$
\begin{aligned}
& \kappa_{[p, k]}(\theta)=\sum_{\ell \in \mathbb{Z}} K_{[p, k]}^{[\ell]} e^{\mathrm{i} \ell \theta}=K_{[p, k]}^{[0]}+\sum_{\ell>0}\left(K_{[p, k]}^{[\ell]} e^{\mathrm{i} \ell \theta}+\left(K_{[p, k]}^{[\ell]}\right)^{T} e^{-\mathrm{i} \ell \theta}\right), \\
& \xi_{[p, k]}(\theta)=\sum_{\ell \in \mathbb{Z}} H_{[p, k]}^{[\ell]} e^{\mathrm{i} \ell \theta}=H_{[p, k]}^{[0]}+\sum_{\ell>0}\left(H_{[p, k]}^{[\ell]} e^{\mathrm{i} \ell \theta}-\left(H_{[p, k]}^{[\ell]}\right)^{T} e^{-\mathrm{i} \ell \theta}\right) \\
& \mu_{[p, k]}(\theta)=\sum_{\ell \in \mathbb{Z}} M_{[p, k]}^{[\ell]} e^{\mathrm{i} \ell \theta}=M_{[p, k]}^{[0]}+\sum_{\ell>0}\left(M_{[p, k]}^{[\ell]} e^{\mathrm{i} \ell \theta}+\left(M_{[p, k]}^{[\ell]}\right)^{T} e^{-\mathrm{i} \ell \theta}\right) .
\end{aligned}
$$

Due to the compact support of the reference B-splines $\beta_{1,[p, k]}, \ldots, \beta_{p-k,[p, k]}$ (see (6.22)), there are only a finite number of nonzero blocks $K_{[p, k]}^{[\ell]}, H_{[p, k]}^{[\ell]}, M_{[p, k]}^{[\ell]}$. Consequently, the series in (6.43)-(6.45) are actually finite sums. We are now ready to state and prove a few lemmas that we shall use in the proof of Theorem 6.14. In what follows, we use the same notation as in Section 6.2 (see Notation 6.3).

Lemma 6.11. Let $p, n \geq 1$ and $0 \leq k \leq p-1$. Then,

$$
\begin{aligned}
& \tilde{A}_{n,[p, k]}(1)=n T_{n-\nu}\left(\kappa_{[p, k]}\right), \\
& \tilde{H}_{n,[p, k]}(1)=T_{n-\nu}\left(\xi_{[p, k]}\right), \\
& \tilde{M}_{n,[p, k]}(1)=n^{-1} T_{n-\nu}\left(\mu_{[p, k]}\right) .
\end{aligned}
$$


Proof. The proof of (6.46) is given in Lemma 6.4. The proofs of (6.47) and (6.48) are essentially the same as the proof of (6.46).

LEMMA 6.12. Let $p \geq 1$ and $0 \leq k \leq p-1$. Then, for all functions $g \in L^{1}([0,1])$,

$$
\begin{gathered}
\left\{n^{-1} \hat{A}_{n,[p, k]}(g)\right\}_{n} \sim_{\mathrm{GLT}} g(x) \kappa_{[p, k]}(\theta), \\
\left\{\hat{H}_{n,[p, k]}(g)\right\}_{n} \sim_{\mathrm{GLT}} g(x) \xi_{[p, k]}(\theta), \\
\left\{n \hat{M}_{n,[p, k]}(g)\right\}_{n} \sim_{\mathrm{GLT}} g(x) \mu_{[p, k]}(\theta) .
\end{gathered}
$$

Proof. Except for the fact that $g$ is replaced by $a$, relation (6.49) is nothing else but (6.27), which has been proved on the basis of (6.46) in Steps 1-3 of the proof of Theorem 6.5. The proofs of (6.50) and (6.51) are completely analogous to the proof of (6.49); they are based on (6.47) and (6.48) instead of (6.46).

The last lemma shows that $H_{n,[p, k]}(g)$ is "almost" skew-symmetric whenever $g$ is continuous. In this regard, we note that $H_{n,[p, k]}(1)$ is skew-symmetric, as it is clear from equation (6.42) with $g=1$ and the fact that, for every $i, j=1, \ldots, n(p-k)+k-1$,

$$
\int_{0}^{1} B_{j+1,[p, k]}^{\prime}(x) B_{i+1,[p, k]}(x) \mathrm{d} x=-\int_{0}^{1} B_{j+1,[p, k]}(x) B_{i+1,[p, k]}^{\prime}(x) \mathrm{d} x .
$$

LeMma 6.13. Let $p, n \geq 1$ and $0 \leq k \leq p-1$. For $i=1, \ldots, n(p-k)+k-1$, let $x_{i}$ be any point in the support of the $B$-spline $B_{i+1,[p, k]}$. Then, for all functions $g \in C([0,1])$,

$$
\begin{aligned}
\left\|H_{n,[p, k]}(g)-\Delta_{n,[p, k]}(g) H_{n,[p, k]}(1)\right\| & \leq C \omega_{g}\left(n^{-1}\right), \\
\left\|H_{n,[p, k]}(g)-H_{n,[p, k]}(1) \Delta_{n,[p, k]}(g)\right\| & \leq C \omega_{g}\left(n^{-1}\right),
\end{aligned}
$$

where $C$ is a constant independent of $n$ and $\Delta_{n,[p, k]}(g)=\operatorname{diag}_{i=1, \ldots, n(p-k)+k-1} g\left(x_{i}\right)$.

Proof. Throughout this proof, the letter $C$ denotes a generic constant independent of $n$. For $i, j=1, \ldots, n(p-k)+k-1$, we have

$$
\begin{aligned}
\left(H_{n,[p, k]}(g)\right)_{i j}= & \int_{0}^{1} g(x) B_{j+1,[p, k]}^{\prime}(x) B_{i+1,[p, k]}(x) \mathrm{d} x \\
= & \int_{\operatorname{supp}\left(B_{i+1,[p, k]}\right)} g(x) B_{j+1,[p, k]}^{\prime}(x) B_{i+1,[p, k]}(x) \mathrm{d} x \\
= & g\left(x_{i}\right) \int_{\operatorname{supp}\left(B_{i+1,[p, k]}\right)} B_{j+1,[p, k]}^{\prime}(x) B_{i+1,[p, k]}(x) \mathrm{d} x \\
& \quad+\int_{\operatorname{supp}\left(B_{i+1,[p, k]}\right)}\left(g(x)-g\left(x_{i}\right)\right) B_{j+1,[p, k]}^{\prime}(x) B_{i+1,[p, k]}(x) \mathrm{d} x \\
= & \left(\Delta_{n,[p, k]}(g)\right)_{i i}\left(H_{n,[p, k]}(1)\right)_{i j}+Z_{i j} .
\end{aligned}
$$

By (6.15) and (6.16)-(6.18),

$$
\begin{aligned}
\left|Z_{i j}\right| & =\left|\int_{\operatorname{supp}\left(B_{i+1,[p, k]}\right)}\left(g(x)-g\left(x_{i}\right)\right) B_{j+1,[p, k]}^{\prime}(x) B_{i+1,[p, k]}(x) \mathrm{d} x\right| \\
& =\int_{\operatorname{supp}\left(B_{i+1,[p, k]}\right)}\left|g(x)-g\left(x_{i}\right)\right|\left|B_{j+1,[p, k]}^{\prime}(x)\right|\left|B_{i+1,[p, k]}(x)\right| \mathrm{d} x \\
& \leq \max _{x \in \operatorname{supp}\left(B_{i+1,[p, k]}\right)}\left|g(x)-g\left(x_{i}\right)\right| C_{p} n \int_{\operatorname{supp}\left(B_{i+1,[p, k]}\right)} \mathrm{d} x \\
& \leq C \omega_{g}\left(n^{-1}\right) .
\end{aligned}
$$


In view of (6.15), the matrix $Z=H_{n,[p, k]}(g)-\Delta_{n,[p, k]}(g) H_{n,[p, k]}(1)$, just as the matrices $H_{n,[p, k]}(g)$ and $H_{n,[p, k]}(1)$, is banded with bandwidth bounded by a constant $C$ independent of $n$. Thus, (6.54) and $\mathbf{N} \mathbf{1}$ imply that $\|Z\| \leq C \omega_{g}\left(n^{-1}\right)$, which proves (6.52). The proof of (6.53) is completely analogous (simply repeat the above steps using $g\left(x_{j}\right)$ in place of $g\left(x_{i}\right)$ and $\operatorname{supp}\left(B_{j+1,[p, k]}\right)$ in place of $\left.\operatorname{supp}\left(B_{i+1,[p, k]}\right)\right)$.

THEOREM 6.14. Let $a_{11}, a_{12}, a_{21}, a_{22} \in L^{1}([0,1]), p \geq 1$, and $0 \leq k \leq p-1$. Then,

$$
\left\{B_{n,[p, k]}(A)\right\}_{n} \sim_{\sigma} \eta_{[p, k]}(x, \theta)=\left[\begin{array}{ll}
a_{11}(x) \kappa_{[p, k]}(\theta) & a_{12}(x) \xi_{[p, k]}(\theta) \\
a_{21}(x) \xi_{[p, k]}(\theta) & a_{22}(x) \mu_{[p, k]}(\theta)
\end{array}\right] .
$$

If moreover $a_{21}=-a_{12} \in C([0,1])$, then we also have

$$
\left\{B_{n,[p, k]}(A)\right\}_{n} \sim_{\lambda} \eta_{[p, k]}(x, \theta) .
$$

Proof. Keeping in mind Notation 6.3, define

$$
\hat{B}_{n,[p, k]}(A)=\left[\begin{array}{cc}
n^{-1} \hat{A}_{n,[p, k]}\left(a_{11}\right) & \hat{H}_{n,[p, k]}\left(a_{12}\right) \\
\hat{H}_{n,[p, k]}\left(a_{21}\right) & n \hat{M}_{n,[p, k]}\left(a_{22}\right)
\end{array}\right] .
$$

Since

$$
\begin{aligned}
& A_{n,[p, k]}\left(a_{11}\right)=P_{n,[p, k]}^{*} \hat{A}_{n,[p, k]}\left(a_{11}\right) P_{n,[p, k]}, \\
& H_{n,[p, k]}\left(a_{12}\right)=P_{n,[p, k]}^{*} \hat{H}_{n,[p, k]}\left(a_{12}\right) P_{n,[p, k]}, \\
& H_{n,[p, k]}\left(a_{21}\right)=P_{n,[p, k]}^{*} \hat{H}_{n,[p, k]}\left(a_{21}\right) P_{n,[p, k]}, \\
& M_{n,[p, k]}\left(a_{22}\right)=P_{n,[p, k]}^{*} \hat{M}_{n,[p, k]}\left(a_{22}\right) P_{n,[p, k]},
\end{aligned}
$$

from (6.41) we obtain

$$
B_{n,[p, k]}(A)=\left[\begin{array}{cc}
P_{n,[p, k]}^{*} & O \\
O & P_{n,[p, k]}^{*}
\end{array}\right] \hat{B}_{n,[p, k]}(A)\left[\begin{array}{cc}
P_{n,[p, k]} & O \\
O & P_{n,[p, k]}
\end{array}\right] .
$$

By Lemma 6.12 and GLT 6 we have $\left\{\hat{B}_{n,[p, k]}(A)\right\}_{n} \sim_{\mathrm{GLT}} \eta_{[p, k]}(x, \theta)$. It follows that $\left\{\hat{B}_{n,[p, k]}(A)\right\}_{n} \sim_{\sigma} \eta_{[p, k]}(x, \theta)$ by GLT 1 and $\left\{B_{n,[p, k]}(A)\right\}_{n} \sim_{\sigma} \eta_{[p, k]}(x, \theta)$ by $\mathbf{S} \mathbf{5}$.

It only remains to prove that $\left\{B_{n,[p, k]}(A)\right\}_{n} \sim_{\lambda} \eta_{[p, k]}(x, \theta)$ under the assumption that $a_{21}=-a_{12} \in C([0,1])$. This assumption ensures that $\hat{B}_{n,[p, k]}(A)$ is "almost" Hermitian. More precisely, if $x_{i}$ is any point in the support of the B-spline $B_{i+1,[p, k]}$, by Lemma 6.13, we have

$$
\hat{B}_{n,[p, k]}(A)=C_{n}+Z_{n},
$$

where

$$
C_{n}=\left[\begin{array}{cc}
n^{-1} \hat{A}_{n,[p, k]}\left(a_{11}\right) & \hat{\Delta}_{n,[p, k]}\left(a_{12}\right) \hat{H}_{n,[p, k]}(1) \\
\hat{H}_{n,[p, k]}(1) \hat{\Delta}_{n,[p, k]}\left(a_{21}\right) & n \hat{M}_{n,[p, k]}\left(a_{22}\right)
\end{array}\right]
$$

is symmetric by the skew-symmetry of $H_{n,[p, k]}(1)$ and the hypothesis $a_{21}=-a_{12}$, and $Z_{n}$ is defined by

$$
Z_{n}=\left[\begin{array}{cc}
O & Y_{n} \\
W_{n} & O
\end{array}\right]
$$


with

$$
\begin{aligned}
Y_{n} & =\hat{H}_{n,[p, k]}\left(a_{12}\right)-\hat{\Delta}_{n,[p, k]}\left(a_{12}\right) \hat{H}_{n,[p, k]}(1), \\
W_{n} & =\hat{H}_{n,[p, k]}\left(a_{21}\right)-\hat{H}_{n,[p, k]}(1) \hat{\Delta}_{n,[p, k]}\left(a_{21}\right) .
\end{aligned}
$$

By Lemma 6.13 and the continuity of $a_{21}=-a_{12}$,

$$
\left\|Z_{n}\right\|=\max \left(\left\|Y_{n}\right\|,\left\|W_{n}\right\|\right) \leq C \omega_{a_{12}}\left(n^{-1}\right) \rightarrow 0 .
$$

The thesis (6.55) now follows from GLT 2, taking into account the decomposition (6.57) and equation (6.56).

6.5. Higher-order isogeometric Galerkin discretization of eigenvalue problems. Let $\mathbb{R}^{+}$be the set of positive real numbers. Consider the following eigenvalue problem: find eigenvalues $\lambda_{j} \in \mathbb{R}^{+}$and eigenfunctions $u_{j}$, for $j=1,2, \ldots, \infty$, such that

$$
\begin{cases}-\left(a(x) u_{j}^{\prime}(x)\right)^{\prime}=\lambda_{j} b(x) u_{j}(x), & x \in \Omega, \\ u_{j}(x)=0, & x \in \partial \Omega,\end{cases}
$$

where $\Omega$ is a bounded open interval in $\mathbb{R}$ and we assume that $a, b \in L^{1}(\Omega)$ and $a, b>0$ a.e. in $\Omega$. It can be shown that the eigenvalues $\lambda_{j}$ must necessarily be real and positive. This can be formally seen by multiplying (6.58) by $u_{j}(x)$ and integrating over $\Omega$ :

$$
\lambda_{j}=\frac{-\int_{\Omega}\left(a(x) u_{j}^{\prime}(x)\right)^{\prime} u_{j}(x) \mathrm{d} x}{\int_{\Omega} b(x)\left(u_{j}(x)\right)^{2} \mathrm{~d} x}=\frac{\int_{\Omega} a(x)\left(u_{j}^{\prime}(x)\right)^{2} \mathrm{~d} x}{\int_{\Omega} b(x)\left(u_{j}(x)\right)^{2} \mathrm{~d} x}>0 .
$$

Isogeometric Galerkin discretization. The weak form of (6.58) reads as follows: find eigenvalues $\lambda_{j} \in \mathbb{R}^{+}$and eigenfunctions $u_{j} \in H_{0}^{1}(\Omega)$, for $j=1,2, \ldots, \infty$, such that

$$
\mathrm{a}\left(u_{j}, w\right)=\lambda_{j}\left(b u_{j}, w\right), \quad \forall w \in H_{0}^{1}(\Omega),
$$

where

$$
\mathrm{a}\left(u_{j}, w\right)=\int_{\Omega} a(x) u_{j}^{\prime}(x) w^{\prime}(x) \mathrm{d} x, \quad\left(b u_{j}, w\right)=\int_{\Omega} b(x) u_{j}(x) w(x) \mathrm{d} x .
$$

In the standard Galerkin method, we fix a set of basis functions $\left\{\varphi_{1}, \ldots, \varphi_{N}\right\} \subset H_{0}^{1}(\Omega)$, we define the so-called approximation space $\mathcal{W}=\operatorname{span}\left(\varphi_{1}, \ldots, \varphi_{N}\right)$, and we find approximations of the exact eigenpairs $\left(\lambda_{j}, u_{j}\right), j=1,2, \ldots, \infty$, by solving the following (Galerkin) problem: find $\lambda_{j, \mathcal{W}} \in \mathbb{R}^{+}$and $u_{j, \mathcal{W}} \in \mathcal{W}$, for $j=1, \ldots, N$, such that

$$
\mathrm{a}\left(u_{j, \mathcal{W}}, w\right)=\lambda_{j, \mathcal{W}}\left(b u_{j, \mathcal{W}}, w\right), \quad \forall w \in \mathcal{W} .
$$

Assuming that the exact and numerical eigenvalues are arranged in non-decreasing order, then the pair $\left(\lambda_{j, \mathcal{W}}, u_{j, \mathcal{W}}\right)$ is taken as an approximation of the pair $\left(\lambda_{j}, u_{j}\right)$, for all $j=1, \ldots, N$, as prescribed in [78, Chapter 6], where one can find an error analysis for such a choice. The numbers $\lambda_{j, \mathcal{W}} / \lambda_{j}-1, j=1, \ldots, N$, are referred to as the (relative) eigenvalue errors. In view of the canonical identification of each function $w \in \mathcal{W}$ with its coefficient vector with respect to the basis $\left\{\varphi_{1}, \ldots, \varphi_{N}\right\}$, solving the Galerkin problem (6.59) is equivalent to solving the generalized eigenvalue problem

$$
A \mathbf{u}_{j, \mathcal{W}}=\lambda_{j, \mathcal{W}} M \mathbf{u}_{j, \mathcal{W}}
$$


where $\mathbf{u}_{j, \mathcal{W}}$ is the coefficient vector of $u_{j, \mathcal{W}}$ with respect to $\left\{\varphi_{1}, \ldots, \varphi_{N}\right\}$ and

$$
\begin{gathered}
A=\left[\mathrm{a}\left(\varphi_{j}, \varphi_{i}\right)\right]_{i, j=1}^{N}=\left[\int_{\Omega} a(x) \varphi_{j}^{\prime}(x) \varphi_{i}^{\prime}(x) \mathrm{d} x\right]_{i, j=1}^{N}, \\
M=\left[\left(b \varphi_{j}, \varphi_{i}\right)\right]_{i, j=1}^{N}=\left[\int_{\Omega} b(x) \varphi_{j}(x) \varphi_{i}(x) \mathrm{d} x\right]_{i, j=1}^{N} .
\end{gathered}
$$

The matrices $A$ and $M$ are referred to as the stiffness and mass matrices, respectively. Due to our assumption that $a, b>0$ a.e. on $\Omega$, both $A$ and $M$ are symmetric positive definite regardless of the chosen basis functions $\varphi_{1}, \ldots, \varphi_{N}$. Moreover, it is clear from (6.60) that the numerical eigenvalues $\lambda_{j, \mathcal{W}}, j=1, \ldots, N$, are just the eigenvalues of the matrix

$$
L=M^{-1} A \text {. }
$$

In the isogeometric Galerkin method [30,61], we assume that the physical domain $\Omega$ is described by a global geometry function $G:[0,1] \rightarrow \bar{\Omega}$, which is invertible and satisfies $G(\partial([0,1]))=\partial \bar{\Omega}$. We fix a set of basis functions $\left\{\hat{\varphi}_{1}, \ldots, \hat{\varphi}_{N}\right\}$ defined on the reference (parametric) domain $[0,1]$ and vanishing on the boundary $\partial([0,1])$, and we find approximations to the exact eigenpairs $\left(\lambda_{j}, u_{j}\right), j=1,2, \ldots, \infty$, by using the standard Galerkin method described above, in which the basis functions $\varphi_{1}, \ldots, \varphi_{N}$ are chosen as

$$
\varphi_{i}(x)=\hat{\varphi}_{i}\left(G^{-1}(x)\right)=\hat{\varphi}_{i}(\hat{x}), \quad x=G(\hat{x}), \quad i=1, \ldots, N .
$$

The resulting stiffness and mass matrices $A$ and $M$ are given by (6.61) and (6.62), with the basis functions $\varphi_{i}$ defined as in (6.63). If we assume that $G$ and $\hat{\varphi}_{i}, i=1, \ldots, N$, are sufficiently regular, then we can apply standard differential calculus to obtain for $A$ and $M$ the following expressions:

$$
\begin{aligned}
A & =\left[\int_{0}^{1} \frac{a(G(\hat{x}))}{\left|G^{\prime}(\hat{x})\right|} \hat{\varphi}_{j}^{\prime}(\hat{x}) \hat{\varphi}_{i}^{\prime}(\hat{x}) \mathrm{d} \hat{x}\right]_{i, j=1}^{N}, \\
M & =\left[\int_{0}^{1} b(G(\hat{x}))\left|G^{\prime}(\hat{x})\right| \hat{\varphi}_{j}(\hat{x}) \hat{\varphi}_{i}(\hat{x}) \mathrm{d} \hat{x}\right]_{i, j=1}^{N} .
\end{aligned}
$$

GLT analysis of the higher-order isogeometric Galerkin matrices. In the higher-order isogeometric Galerkin approach, a suitable choice of basis functions $\hat{\varphi}_{1}, \ldots, \hat{\varphi}_{N}$ is as follows:

$$
\hat{\varphi}_{i}=B_{i+1,[p, k]}, \quad i=1, \ldots, n(p-k)+k-1,
$$

where $B_{1,[p, k]}, \ldots, B_{n(p-k)+k+1,[p, k]}$ are the $p$-degree $C^{k} \mathrm{~B}$-splines introduced in Section 6.2. If, for any functions $\alpha, \beta \in L^{1}([0,1])$, we define

$$
\begin{aligned}
& A_{n,[p, k]}(\alpha)=\left[\int_{0}^{1} \alpha(\hat{x}) B_{j+1,[p, k]}^{\prime}(\hat{x}) B_{i+1,[p, k]}^{\prime}(\hat{x}) \mathrm{d} \hat{x}\right]_{i, j=1}^{n(p-k)+k-1}, \\
& M_{n,[p, k]}(\beta)=\left[\int_{0}^{1} \beta(\hat{x}) B_{j+1,[p, k]}(\hat{x}) B_{i+1,[p, k]}(\hat{x}) \mathrm{d} \hat{x}\right]_{i, j=1}^{n(p-k)+k-1},
\end{aligned}
$$

then the stiffness and mass matrices (6.64) and (6.65) resulting from the choice of the basis functions as in (6.66) are nothing else but $A_{n,[p, k]}\left(a_{G}\right)$ and $M_{n,[p, k]}\left(b_{G}\right)$, where

$$
a_{G}(\hat{x})=\frac{a(G(\hat{x}))}{\left|G^{\prime}(\hat{x})\right|}, \quad b_{G}(\hat{x})=b(G(\hat{x}))\left|G^{\prime}(\hat{x})\right| .
$$


The main result of this section is Theorem 6.17. It provides formal mathematical proofs to the main results that are discussed in the engineering review [57] by giving the spectral distribution of the normalized sequences

$$
\left\{n^{-1} A_{n,[p, k]}\left(a_{G}\right)\right\}_{n}, \quad\left\{n M_{n,[p, k]}\left(b_{G}\right)\right\}_{n}, \quad\left\{n^{-2} L_{n,[p, k]}\left(a_{G}, b_{G}\right)\right\}_{n},
$$

where

$$
L_{n,[p, k]}\left(a_{G}, b_{G}\right)=\left(M_{n,[p, k]}\left(b_{G}\right)\right)^{-1} A_{n,[p, k]}\left(a_{G}\right)
$$

is the matrix whose eigenvalues are just the numerical eigenvalues produced by the considered higher-order isogeometric Galerkin method. To prove Theorem 6.17, some preliminary work is necessary. In what follows, we use the same notation as in Section 6.2 (see Notation 6.3).

LEMMA 6.15. Let $p \geq 1$ and $0 \leq k \leq p-1$. Then, for all functions $g \in L^{1}([0,1])$,

$$
\begin{aligned}
\left\{n^{-1} \hat{A}_{n,[p, k]}(g)\right\}_{n} & \sim_{\mathrm{GLT}} g(\hat{x}) \kappa_{[p, k]}(\theta), \\
\left\{n \hat{M}_{n,[p, k]}(g)\right\}_{n} & \sim_{\mathrm{GLT}} g(\hat{x}) \mu_{[p, k]}(\theta) .
\end{aligned}
$$

Proof. See Lemma 6.12.

Lemma 6.16. Let $p \geq 1$ and $0 \leq k \leq p-1$. Then $\mu_{[p, k]}(\theta)$ is Hermitian positive definite for all $\theta \in[-\pi, \pi]$.

Proof. By Lemma 6.15,

$$
\left\{n \hat{M}_{n,[p, k]}(1)\right\}_{n} \sim_{\mathrm{GLT}} \mu_{[p, k]}(\theta),
$$

and since $n \hat{M}_{n,[p, k]}(1)$ is symmetric, we infer from GLT 1 that

$$
\left\{n \hat{M}_{n,[p, k]}(1)\right\}_{n} \sim_{\lambda} \mu_{[p, k]}(\theta) .
$$

By (6.19), for every $\mathbf{y} \in \mathbb{R}^{n(p-k)+k-1}$, we have

$$
\begin{aligned}
\mathbf{y}^{T}\left(n M_{n,[p, k]}(1)\right) \mathbf{y} & =n \int_{0}^{1}\left(\sum_{i=1}^{n(p-k)+k-1} y_{i} B_{i+1,[p, k]}(\hat{x})\right)^{2} \mathrm{~d} \hat{x} \\
& =n\left\|\sum_{i=1}^{n(p-k)+k-1} y_{i} B_{i+1,[p, k]}\right\|_{L^{2}([0,1])}^{2} \geq c_{p}\|\mathbf{y}\|^{2} .
\end{aligned}
$$

Hence, by the minimax principle for eigenvalues [13, Corollary III.1.2],

$$
\lambda_{\min }\left(n M_{n,[p, k]}(1)\right)=\min _{\mathbf{y} \neq \mathbf{0}} \frac{\mathbf{y}^{T}\left(n M_{n,[p, k]}(1)\right) \mathbf{y}}{\|\mathbf{y}\|^{2}} \geq c_{p}
$$

for all $n$, which implies that

$$
\lambda_{\min }\left(n \hat{M}_{n,[p, k]}(1)\right) \geq \min \left(c_{p}, 1\right)
$$

for all $n$. Taking into account that $\lambda_{\min }\left(\mu_{[p, k]}(\theta)\right)$ is a continuous function of $\theta$ just as $\mu_{[p, k]}(\theta)$, by (6.67), (6.68), and $\mathbf{S} 3$ we have

$$
\lambda_{\min }\left(\mu_{[p, k]}(\theta)\right) \geq \min \left(c_{p}, 1\right)
$$


for almost every $\theta \in[-\pi, \pi]$, that is, for all $\theta \in[-\pi, \pi]$, thanks to the continuity of $\lambda_{\min }\left(\mu_{[p, k]}(\theta)\right)$. We then conclude that $\mu_{[p, k]}(\theta)$ is Hermitian positive definite for all $\theta \in[-\pi, \pi]$.

THEOREM 6.17. Let $\Omega$ be a bounded open interval in $\mathbb{R}$, and let $a, b \in L^{1}(\Omega)$ with $a, b>0$ a.e. Let $p \geq 1$ and $0 \leq k \leq p-1$. Let $G:[0,1] \rightarrow \bar{\Omega}$ be such that $G^{\prime} \neq 0$ a.e. in $[0,1]$ and

$$
a_{G}(\hat{x})=\frac{a(G(\hat{x}))}{\left|G^{\prime}(\hat{x})\right|} \in L^{1}([0,1])
$$

Then,

$$
\begin{aligned}
&\left\{n^{-1} A_{n,[p, k]}\left(a_{G}\right)\right\}_{n} \sim_{\sigma, \lambda} a_{G}(\hat{x}) \kappa_{[p, k]}(\theta)=\frac{a(G(\hat{x}))}{\left|G^{\prime}(\hat{x})\right|} \kappa_{[p, k]}(\theta), \\
&\left\{n M_{n,[p, k]}\left(b_{G}\right)\right\}_{n} \sim_{\sigma, \lambda} b_{G}(\hat{x}) \mu_{[p, k]}(\theta)=b(G(\hat{x}))\left|G^{\prime}(\hat{x})\right| \mu_{[p, k]}(\theta), \\
&\left\{n^{-2} L_{n,[p, k]}\left(a_{G}, b_{G}\right)\right\}_{n} \sim_{\sigma, \lambda}\left(b_{G}(\hat{x}) \mu_{[p, k]}(\theta)\right)^{-1}\left(a_{G}(\hat{x}) \kappa_{[p, k]}(\theta)\right) \\
&=\frac{a(G(\hat{x}))}{b(G(\hat{x}))\left(G^{\prime}(\hat{x})\right)^{2}}\left(\mu_{[p, k]}(\theta)\right)^{-1} \kappa_{[p, k]}(\theta) .
\end{aligned}
$$

Proof. We first note that it is enough to prove (6.69)-(6.71) with $A_{n,[p, k]}, M_{n,[p, k]}$, $L_{n,[p, k]}$ replaced by, respectively, $\hat{A}_{n,[p, k]}, \hat{M}_{n,[p, k]}, \hat{L}_{n,[p, k]}$, that is,

$$
\begin{aligned}
\left\{n^{-1} \hat{A}_{n,[p, k]}\left(a_{G}\right)\right\}_{n} & \sim_{\sigma, \lambda} a_{G}(\hat{x}) \kappa_{[p, k]}(\theta)=\frac{a(G(\hat{x}))}{\left|G^{\prime}(\hat{x})\right|} \kappa_{[p, k]}(\theta), \\
\left\{n \hat{M}_{n,[p, k]}\left(b_{G}\right)\right\}_{n} & \sim_{\sigma, \lambda} b_{G}(\hat{x}) \mu_{[p, k]}(\theta)=b(G(\hat{x}))\left|G^{\prime}(\hat{x})\right| \mu_{[p, k]}(\theta), \\
\left\{n^{-2} \hat{L}_{n,[p, k]}\left(a_{G}, b_{G}\right)\right\}_{n} \sim_{\sigma, \lambda} & \left(b_{G}(\hat{x}) \mu_{[p, k]}(\theta)\right)^{-1}\left(a_{G}(\hat{x}) \kappa_{[p, k]}(\theta)\right) \\
& =\frac{a(G(\hat{x}))}{b(G(\hat{x}))\left(G^{\prime}(\hat{x})\right)^{2}}\left(\mu_{[p, k]}(\theta)\right)^{-1} \kappa_{[p, k]}(\theta) .
\end{aligned}
$$

Moreover, (6.72) and (6.73) follow immediately from Lemma 6.15 and the symmetry of $\hat{A}_{n,[p, k]}\left(a_{G}\right)$ and $\hat{M}_{n,[p, k]}\left(b_{G}\right)$. It only remains to prove (6.74). The first observation is that

$$
\begin{aligned}
n^{-2} \hat{L}_{n,[p, k]}\left(a_{G}, b_{G}\right) & =\left(n \hat{M}_{n,[p, k]}\left(b_{G}\right)\right)^{-1}\left(n^{-1} \hat{A}_{n,[p, k]}\left(a_{G}\right)\right) \\
& \sim\left(n \hat{M}_{n,[p, k]}\left(b_{G}\right)\right)^{-1 / 2}\left(n^{-1} \hat{A}_{n,[p, k]}\left(a_{G}\right)\right)\left(n \hat{M}_{n,[p, k]}\left(b_{G}\right)\right)^{-1 / 2},
\end{aligned}
$$

where $X \sim Y$ means that the matrix $X$ is similar to $Y$; note that $\hat{M}_{n,[p, k]}\left(b_{G}\right)$ is positive definite because $b_{G}>0$ a.e. in $[0,1]$ by the assumptions on $b$ and $G$, hence $\left(\hat{M}_{n,[p, k]}\left(b_{G}\right)\right)^{-1 / 2}$ is well-defined. By combining the equality in (6.75) with Lemmas 6.15, 6.16, and GLT 4, we immediately obtain

$$
\left\{n^{-2} \hat{L}_{n,[p, k]}\left(a_{G}, b_{G}\right)\right\}_{n} \sim_{\mathrm{GLT}}\left(b_{G}(\hat{x}) \mu_{[p, k]}(\theta)\right)^{-1}\left(a_{G}(\hat{x}) \kappa_{[p, k]}(\theta)\right) .
$$

The singular value distribution in (6.74) follows from (6.76) and GLT 1. Moreover, by Lemmas 6.15, 6.16, GLT 4, and GLT 5 (applied with $f(z)=|z|^{1 / 2}$ ), we have

$$
\begin{aligned}
& \left\{\left(n \hat{M}_{n,[p, k]}\left(b_{G}\right)\right)^{-1 / 2}\left(n^{-1} \hat{A}_{n,[p, k]}\left(a_{G}\right)\right)\left(n \hat{M}_{n,[p, k]}\left(b_{G}\right)\right)^{-1 / 2}\right\}_{n} \\
& \quad \sim_{\mathrm{GLT}}\left(b_{G}(\hat{x}) \mu_{[p, k]}(\theta)\right)^{-1 / 2}\left(a_{G}(\hat{x}) \kappa_{[p, k]}(\theta)\right)\left(b_{G}(\hat{x}) \mu_{[p, k]}(\theta)\right)^{-1 / 2} .
\end{aligned}
$$




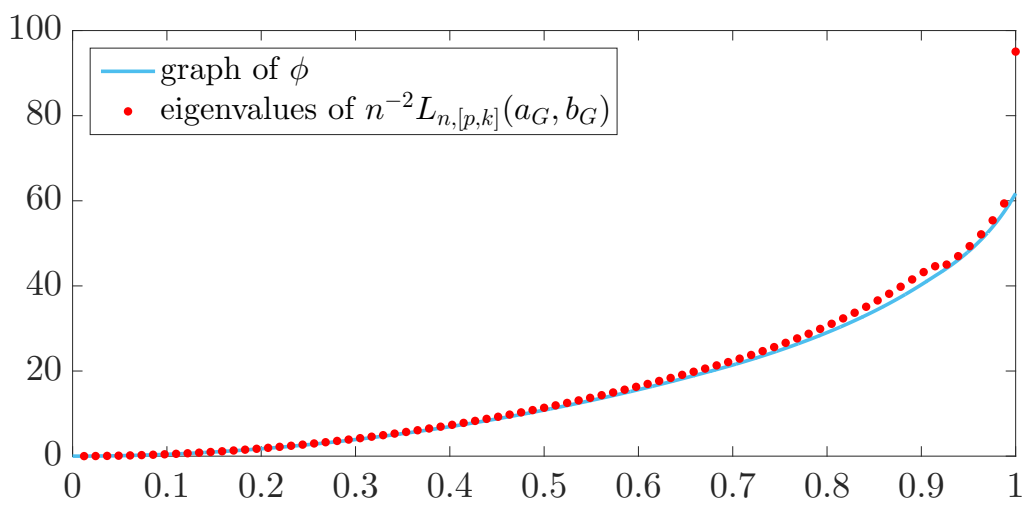

FIG. 6.6. Comparison between the spectrum of the matrix $n^{-2} L_{n,[p, k]}\left(a_{G}, b_{G}\right)$ and the rearranged version $\phi$ of the symbol $\left(b_{G}(\hat{x}) \mu_{[p, k]}(\theta)\right)^{-1}\left(a_{G}(\hat{x}) \kappa_{[p, k]}(\theta)\right)$ for $a(x)=1+x, b(x)=2-\sin (3 x), G(\hat{x})=\hat{x}$, and $p=5, k=3, n=40$.

Considering that $\left(n \hat{M}_{n,[p, k]}\left(b_{G}\right)\right)^{-1 / 2}\left(n^{-1} \hat{A}_{n,[p, k]}\left(a_{G}\right)\right)\left(n \hat{M}_{n,[p, k]}\left(b_{G}\right)\right)^{-1 / 2}$ is symmetric, from GLT 1 we get

$$
\begin{aligned}
& \left\{\left(n \hat{M}_{n,[p, k]}\left(b_{G}\right)\right)^{-1 / 2}\left(n^{-1} \hat{A}_{n,[p, k]}\left(a_{G}\right)\right)\left(n \hat{M}_{n,[p, k]}\left(b_{G}\right)\right)^{-1 / 2}\right\}_{n} \\
& \quad \sim_{\lambda}\left(b_{G}(\hat{x}) \mu_{[p, k]}(\theta)\right)^{-1 / 2}\left(a_{G}(\hat{x}) \kappa_{[p, k]}(\theta)\right)\left(b_{G}(\hat{x}) \mu_{[p, k]}(\theta)\right)^{-1 / 2}
\end{aligned}
$$

which is equivalent to

$$
\begin{aligned}
& \left\{\left(n \hat{M}_{n,[p, k]}\left(b_{G}\right)\right)^{-1 / 2}\left(n^{-1} \hat{A}_{n,[p, k]}\left(a_{G}\right)\right)\left(n \hat{M}_{n,[p, k]}\left(b_{G}\right)\right)^{-1 / 2}\right\}_{n} \\
& \quad \sim_{\lambda}\left(b_{G}(\hat{x}) \mu_{[p, k]}(\theta)\right)^{-1}\left(a_{G}(\hat{x}) \kappa_{[p, k]}(\theta)\right)
\end{aligned}
$$

by Definition 2.8, since

$$
\begin{aligned}
& \left(b_{G}(\hat{x}) \mu_{[p, k]}(\theta)\right)^{-1}\left(a_{G}(\hat{x}) \kappa_{[p, k]}(\theta)\right) \\
& \quad \sim\left(b_{G}(\hat{x}) \mu_{[p, k]}(\theta)\right)^{-1 / 2}\left(a_{G}(\hat{x}) \kappa_{[p, k]}(\theta)\right)\left(b_{G}(\hat{x}) \mu_{[p, k]}(\theta)\right)^{-1 / 2}
\end{aligned}
$$

for all $(\hat{x}, \theta) \in[0,1] \times[-\pi, \pi]$. In view of the similarity in (6.75), we conclude that the eigenvalue distribution in (6.74) is satisfied.

EXAMPLE 6.18. Let us denote by $\phi$ the canonical rearranged version of the symbol $\left(b_{G}(\hat{x}) \mu_{[p, k]}(\theta)\right)^{-1}\left(a_{G}(\hat{x}) \kappa_{[p, k]}(\theta)\right)$ obtained as the limit of the piecewise linear functions $\phi_{\rho}$, according to the construction in Chapter 5 (fifth paragraph). Figure 6.6 displays the graph of $\phi$ and the eigenvalues $\lambda_{1}, \ldots, \lambda_{n(p-k)+k-1}$ of $n^{-2} L_{n,[p, k]}\left(a_{G}, b_{G}\right)$ for $a(x)=1+x$, $b(x)=2-\sin (3 x), G(\hat{x})=\hat{x}$, and $p=5, k=3, n=40$. The graph of $\phi$ has been obtained by plotting the graph of $\phi_{\rho}$ corresponding to a large value of $\rho$. The eigenvalues of $n^{-2} L_{n,[p, k]}\left(a_{G}, b_{G}\right)$ have been sorted in non-decreasing order and placed at the points $\left(t_{q}, \lambda_{q}\right)$ with $t_{q}=\frac{q}{n(p-k)+k-1}, q=1, \ldots, n(p-k)+k-1$. We clearly see from the figure an excellent agreement between $\phi$ and the eigenvalues of $n^{-2} L_{n,[p, k]}\left(a_{G}, b_{G}\right)$. However, we also note the presence of an outlier at the right end of the spectrum.

The reader who is interested in the engineering implications of Theorem 6.17 is referred to the recent review [57]. 


\section{REFERENCES}

[1] A. S. Al-Fhaid, S. Serra-Capizzano, D. Sesana, And M. Z. Ullah, Singular-value (and eigenvalue) distribution and Krylov preconditioning of sequences of sampling matrices approximating integral operators, Numer. Linear Algebra Appl., 21 (2014), pp. 722-743.

[2] F. Avram, On bilinear forms in Gaussian random variables and Toeplitz matrices, Probab. Theory Related Fields, 79 (1988), pp. 37-45.

[3] G. BARBARINO, Equivalence between GLT sequences and measurable functions, Linear Algebra Appl., 529 (2017), pp. 397-412.

[4] - Spectral measures, in Structured Matrices in Numerical Linear Algebra, D. Bini, F. Di Benedetto, E. Tyrtyshnikov, and M. Van Barel, eds., Springer INdAM Series 30, Springer, Cham, 2019, pp. 1-24.

[5] - Normal form for GLT sequences, Preprint on arXiv, 2018. https://arxiv.org/abs/1805.08708v2

[6] - Diagonal matrix sequences and their spectral symbols, Preprint on arXiv, 2017. https://arxiv.org/abs/1710.00810

[7] G. BARBARINO AND C. GARONI, From convergence in measure to convergence of matrix-sequences through concave functions and singular values, Electron. J. Linear Algebra, 32 (2017), pp. 500-513.

[8] G. BARBARINO AND S. SerRA-CAPIZZANO, Non-Hermitian perturbations of Hermitian matrix-sequences and applications to the spectral analysis of approximated PDEs, Numer. Linear Algebra Appl., to appear.

[9] B. BECKERMANN AND A. B. J. KUIJLAARS, Superlinear convergence of conjugate gradients, SIAM J. Numer. Anal., 39 (2001), pp. 300-329.

[10] B. BECKERMANN AND S. SERRA-CAPIZZANO, On the asymptotic spectrum of finite element matrix sequences, SIAM J. Numer. Anal., 45 (2007), pp. 746-769.

[11] P. Benedusi, C. Garoni, R. Krause, X. Li, and S. Serra-Capizzano, Space-time FE-DG discretization of the anisotropic diffusion equation in any dimension: the spectral symbol, SIAM J. Matrix Anal. Appl., 39 (2018), pp. 1383-1420.

[12] D. Bertaccini, M. Donatelli, F. Durastante, and S. Serra-Capizzano, Optimizing a multigrid Runge-Kutta smoother for variable-coefficient convection-diffusion equations, Linear Algebra Appl., 533 (2017), pp. 507-535.

[13] R. Bhatia, Matrix Analysis, Springer, New York, 1997.

[14] D. Bini, B. IANnAZzo, AND B. Meini, Numerical Solution of Algebraic Riccati Equations, SIAM, Philadelphia, 2012.

[15] D. Bini, G. LATouche, AND B. Meini, Numerical Methods for Structured Markov Chains, Oxford University Press, New York, 2005.

[16] V. I. BogacheV, Measure Theory. Vol. I., Springer, Berlin, 2007.

[17] J. M. Bogoya, A. Böttcher, S. M. GrudSKy, AND E. A. MAXimenko, Maximum norm versions of the Szegó and Avram-Parter theorems for Toeplitz matrices, J. Approx. Theory, 196 (2015), pp. 79-100.

[18] J. M. Bogoya, A. BötTCher, And E. A. Maximenko, From convergence in distribution to uniform convergence, Bol. Soc. Mat. Mex., 22 (2016), pp. 695-710.

[19] J. M. Bogoya, S. M. GRUdSKy, AND E. A. MAXIMENKo, Eigenvalues of Hermitian Toeplitz matrices generated by simple-loop symbols with relaxed smoothness, in Large Truncated Toeplitz Matrices, Toeplitz Operators, and Related Topics, D. A. Bini, T. Ehrhardt, A. Yu. Karlovich, and I. Spitkovsky, eds, Oper. Theory Adv. Appl., 259, Birkhäuser, Basel, 2017, pp. 179-212.

[20] A. BötTCher, C. GARONI, AND S. SERRA-CAPIZZANo, Exploration of Toeplitz-like matrices with unbounded symbols is not a purely academic journey, Sb. Math., 208 (2017), pp. 1602-1627.

[21] A. BötTCher AND S. M. GRUdSKy, Toeplitz Matrices, Asymptotic Linear Algebra, and Functional Analysis, Birkhäuser, Basel, 2000

[22] - Spectral Properties of Banded Toeplitz Matrices, SIAM, Philadelphia, 2005.

[23] A. BötTCher AND B. Silbermann, Introduction to Large Truncated Toeplitz Matrices, Springer, New York, 1999.

[24] - Analysis of Toeplitz Operators, 2nd ed., Springer, Berlin, 2006.

[25] H. BRezIs, Functional Analysis, Sobolev Spaces and Partial Differential Equations, Springer, New York, 2011.

[26] J.-F. CAI, R. H. CHAN, AND Z. W. SHEN, A framelet-based image inpainting algorithm, Appl. Comput. Harmon. Anal., 24 (2008), pp. 131-149.

[27] O. Caramello, Theories, Sites, Toposes, Oxford University Press, Oxford, 2018.

[28] A. Cicone, C. GARoni, AND S. SERra-CAPIZZAno, Spectral and convergence analysis of the Discrete ALIF method, Linear Algebra Appl., 580 (2019), pp. 62-95.

[29] E. CinlaR, Introduction to Stochastic Processes, Prentice-Hall, Englewood Cliffs, 1975.

[30] J. A. Cottrell, T. J. R. Hughes, AND Y. Bazilevs, Isogeometric Analysis, Wiley, Chichester, 2009.

[31] C. De Boor, A Practical Guide to Splines, revised ed., Springer, New York, 2001.

[32] V. Del Prete, F. Di Benedetto, M. Donatelli, and S. Serra-Capizzano, Symbol approach in 
a signal-restoration problem involving block Toeplitz matrices, J. Comput. Appl. Math., 272 (2014), pp. 399-416.

[33] F. Di Benedetto, G. Fiorentino, and S. Serra-Capizzano, CG preconditioning for Toeplitz matrices, Comput. Math. Appl., 25 (1993), pp. 33-45.

[34] M. Donatelli, C. Garoni, C. Manni, S. Serra-Capizzano, and H. Speleers, Robust and optimal multi-iterative techniques for IgA Galerkin linear systems, Comput. Methods Appl. Mech. Engrg., 284 (2015), pp. 230-264.

[35] - Robust and optimal multi-iterative techniques for IgA collocation linear systems, Comput. Methods Appl. Mech. Engrg., 284 (2015), pp. 1120-1146.

[36] - Spectral analysis and spectral symbol of matrices in isogeometric collocation methods, Math. Comp., 85 (2016), pp. 1639-1680.

[37] - Symbol-based multigrid methods for Galerkin B-spline isogeometric analysis, SIAM J. Numer. Anal., 55 (2017), pp. 31-62.

[38] M. Donatelli, C. Garoni, M. Mazza, S. Serra-Capizzano, And D. Sesana, Spectral behavior of preconditioned non-Hermitian multilevel block Toeplitz matrices with matrix-valued symbol, Appl. Math. Comput., 245 (2014), pp. 158-173.

[39] - Preconditioned HSS method for large multilevel block Toeplitz linear systems via the notion of matrix-valued symbol, Numer. Linear Algebra Appl., 23 (2016), pp. 83-119.

[40] M. Donatelli, M. Mazza, And S. SerRA-CAPIZZAno, Spectral analysis and structure preserving preconditioners for fractional diffusion equations, J. Comput. Phys., 307 (2016), pp. 262-279.

[41] M. Donatelli, M. Neytcheva, And S. Serra-Capizzano, Canonical eigenvalue distribution of multilevel block Toeplitz sequences with non-Hermitian symbols, in Spectral Theory, Mathematical System Theory, Evolution Equations, Differential and Difference Equations, W. Arendt, J. A. Ball, J. Behrndt, K.-H. Förster, V. Mehrmann, and C. Trunk, eds., Oper. Theory Adv. Appl., 221, Birkhäuser, Basel, 2012, pp. 269-291.

[42] A. Dorostkar, M. Neytcheva, And S. Serra-CapizZAno, Spectral analysis of coupled PDEs and of their Schur complements via generalized locally Toeplitz sequences in 2D, Comput. Methods Appl. Mech. Engrg., 309 (2016), pp. 74-105.

[43] M. Dumbser, F. Fambri, I. Furci, M. Mazza, S. Serra-Capizzano, And M. Tavelli, Staggered discontinuous Galerkin methods for the incompressible Navier-Stokes equations: spectral analysis and computational results, Numer. Linear Algebra Appl., 25 (2018), Art. e2151, 31 pages.

[44] S.-E. Ekström, I. FURCI, AND S. SERRA-CAPIZZANo, Exact formulae and matrix-less eigensolvers for block banded symmetric Toeplitz matrices, BIT, 58 (2018), pp. 937-968.

[45] C. GARONI, Spectral distribution of PDE discretization matrices from isogeometric analysis: the case of $L^{1}$ coefficients and non-regular geometry, J. Spectr. Theory, 8 (2018), pp. 297-313.

[46] C. Garoni, C. Manni, F. Pelosi, S. Serra-Capizzano, and H. Speleers, On the spectrum of stiffness matrices arising from isogeometric analysis, Numer. Math., 127 (2014), pp. 751-799.

[47] C. Garoni, C. Manni, S. Serra-Capizzano, D. Sesana, And H. Speleers, Lusin theorem, GLT sequences and matrix computations: an application to the spectral analysis of PDE discretization matrices, J. Math. Anal. Appl., 446 (2017), pp. 365-382.

[48] - Spectral analysis and spectral symbol of matrices in isogeometric Galerkin methods, Math. Comp., 86 (2017), pp. 1343-1373.

[49] C. GAroni, M. MAZZA, And S. SERra-CAPIZZAno, Block generalized locally Toeplitz sequences: from the theory to the applications, Axioms, 7 (2018), Art. 49, 29 pages.

[50] C. Garoni And S. SERrA-CAPIZZAno, Generalized Locally Toeplitz Sequences: Theory and Applications. Vol. I., Springer, Cham, 2017.

[51] - Generalized Locally Toeplitz Sequences: Theory and Applications. Vol. II., Springer, Cham, 2018.

[52] — Block generalized locally Toeplitz sequences: the case of matrix functions and an engineering application, Electron. J. Linear Algebra, 35 (2019), pp. 204-222.

[53] C. Garoni, S. Serra-Capizzano, and D. Sesana, Tools for determining the asymptotic spectral distribution of non-Hermitian perturbations of Hermitian matrix-sequences and applications, Integral Equat. Oper. Theory, 81 (2015), pp. 213-225.

[54] - Spectral analysis and spectral symbol of d-variate $\mathbb{Q}_{\boldsymbol{p}}$ Lagrangian FEM stiffness matrices, SIAM J. Matrix Anal. Appl., 36 (2015), pp. 1100-1128.

[55] - Block locally Toeplitz sequences: construction and properties, in Structured Matrices in Numerical Linear Algebra, D. Bini, F. Di Benedetto, E. Tyrtyshnikov, and M. Van Barel, eds., Springer INdAM Series 30, Springer, Cham, 2019, pp. 25-58.

[56] - Block generalized locally Toeplitz sequences: topological construction, spectral distribution results, and star-algebra structure, in Structured Matrices in Numerical Linear Algebra, D. Bini, F. Di Benedetto, E. Tyrtyshnikov, and M. Van Barel, eds., Springer INdAM Series 30, Springer, Cham, 2019, pp. 59-79.

[57] C. Garoni, H. Speleers, S.-E. Ekström, A. Reali, S. Serra-Capizzano, and T. J. R. Hughes, Symbol-based analysis of finite element and isogeometric B-spline discretizations of eigevalue problems: 
exposition and review, Arch. Comput. Methods Engrg., 26 (2019), pp. 1639-1690.

[58] L. GOLINSKII AND S. SERRA-CAPIZZANO, The asymptotic properties of the spectrum of nonsymmetrically perturbed Jacobi matrix sequences, J. Approx. Theory, 144 (2007), pp. 84-102.

[59] U. Grenander And G. SZegô, Toeplitz Forms and Their Applications, 2nd ed., Chelsea, New York, 1984.

[60] L. HÖRMANDER, Pseudo-differential operators and non-elliptic boundary problems, Ann. of Math., 83 (1966), pp. 129-209.

[61] T. J. R. Hughes, J. A. CotTrell, AND Y. BAZILEVs, Isogeometric analysis: CAD, finite elements, NURBS, exact geometry and mesh refinement, Comput. Methods Appl. Mech. Engrg., 194 (2005), pp. 4135-4195.

[62] M. MaZZA, A. RATNANi, AND S. SERRA-CAPIZZANo, Spectral analysis and spectral symbol for the $2 D$ curl-curl (stabilized) operator with applications to the related iterative solutions, Math. Comp., 88 (2019), pp. $1155-1188$.

[63] M. NEUTs, Structured Stochastic Matrices of M/G/1 Type and Their Applications, Marcel Dekker, New York, 1989.

[64] S. V. PARTER, On the distribution of the singular values of Toeplitz matrices, Linear Algebra Appl., 80 (1986), pp. 115-130.

[65] J. Peters And U. ReIF, Subdivision Surfaces, Springer, Berlin, Heidelberg, 2008.

[66] A. Quarteroni, Numerical Models for Differential Problems, 3rd. ed., Springer, Cham, 2017.

[67] R. RAHLA, S. SERRA-CAPIZZANo, C. TABLINo-Possio, Spectral analysis of $\mathbb{P}_{k}$ finite element matrices in the case of Friedrichs-Keller triangulations via GLT technology, Numer. Linear Algebra Appl., to appear.

[68] F. Roman, C. MANNI, AND H. SPELEERS, Spectral analysis of matrices in Galerkin methods based on generalized B-splines with high smoothness, Numer. Math., 135 (2017), pp. 169-216.

[69] H. L. Royden And P. M. FitzPatrick, Real Analysis, 4th ed., Pearson, New York, 2010.

[70] W. Rudin, Principles of Mathematical Analysis, 3rd ed., McGraw-Hill, New York, 1976.

[71] - Real and Complex Analysis, 3rd ed., McGraw-Hill, New York, 1987.

[72] E. Salinelli, S. SerRa-CAPIZZANo, AND D. SeSANA, Eigenvalue-eigenvector structure of SchoenmakersCoffey matrices via Toeplitz technology and applications, Linear Algebra Appl., 491 (2016), pp. 138-160.

[73] L. L. SCHUMAKER, Spline Functions: Basic Theory, 3rd ed., Cambridge University Press, Cambridge, 2007.

[74] S. SERRA-CAPIZZANO, More inequalities and asymptotics for matrix valued linear positive operators: the noncommutative case, in Toeplitz Matrices and Singular Integral Equations, A. Böttcher, I. Gohberg, and P. Junghanns, eds., Oper. Theory Adv. Appl., 135, Birkhäuser, Basel, 2002, pp. 293-315.

[75] __ Generalized locally Toeplitz sequences: spectral analysis and applications to discretized partial differential equations, Linear Algebra Appl., 366 (2003), pp. 371-402.

[76] _ - The GLT class as a generalized Fourier analysis and applications, Linear Algebra Appl., 419 (2006), pp. 180-233.

[77] S. SERRA-CAPIZZANO AND P. TILLI, On unitarily invariant norms of matrix-valued linear positive operators, J. Inequal. Appl., 7 (2002), pp. 309-330.

[78] G. StRAng AND G. FIX, An Analysis of the Finite Element Method, 2nd. ed., Wellesley-Cambridge, Wellesley, 2008.

[79] P. TILLI, Locally Toeplitz sequences: spectral properties and applications, Linear Algebra Appl., 278 (1998), pp. 91-120.

[80] - A note on the spectral distribution of Toeplitz matrices, Linear and Multilinear Algebra, 45 (1998), pp. 147-159.

[81] - Some results on complex Toeplitz eigenvalues, J. Math. Anal. Appl., 239 (1999), pp. 390-401.

[82] E. E. TYRTYSHNIKOV, A unifying approach to some old and new theorems on distribution and clustering, Linear Algebra Appl., 232 (1996), pp. 1-43.

[83] E. E. TyRTYShNikov AND N. L. ZamaRASHKin, Spectra of multilevel Toeplitz matrices: advanced theory via simple matrix relationships, Linear Algebra Appl., 270 (1998), pp. 15-27.

[84] N. L. ZAMARASHKIN AND E. E. TYRTYSHNIKOv, Distribution of eigenvalues and singular values of Toeplitz matrices under weakened conditions on the generating function, Sb. Math., 188 (1997), pp. 1191-1201. 